\title{
Designing Multidimensional Constellations and Efficient Detection Schemes for Sparse Code Multiple Access (SCMA) Systems
}

\author{
by \\ Monirosharieh Vameghestahbanati, M.Sc. \\ A dissertation submitted to the \\ Faculty of Graduate and Postdoctoral Affairs \\ in partial fulfillment of the requirements for the degree of
}

Doctor of Philosophy in Electrical and Computer Engineering

Ottawa-Carleton Institute for Electrical and Computer Engineering

Department of Systems and Computer Engineering

Carleton University

Ottawa, Ontario

January 2020

(C) Copyright

Monirosharieh Vameghestahbanati, 2020 
The undersigned hereby recommends to the

Faculty of Graduate and Postdoctoral Affairs acceptance of the dissertation

\section{Designing Multidimensional Constellations and Efficient Detection Schemes for Sparse Code Multiple Access (SCMA) Systems}

submitted by Monirosharieh Vameghestahbanati, M.Sc.

in partial fulfillment of the requirements for the degree of

Doctor of Philosophy in Electrical and Computer Engineering

Professor Ian Marsland, Thesis Co-supervisor

Professor Halim Yanikomeroglu, Thesis Co-supervisor

Professor Ramy Gohary, Thesis Co-supervisor

Ottawa-Carleton Institute for Electrical and Computer Engineering

Department of Systems and Computer Engineering

Carleton University

January, 2020 


\section{Abstract}

Sparse code multiple access (SCMA) is a class of non-orthogonal multiple access that is proposed to support uplink machine-type communication services. In this thesis, we investigate the design of SCMA systems from two main aspects: multidimensional constellations (MdCs) and efficient detection schemes.

In an SCMA system, designing MdCs plays an important role in the system performance. Since the behavior of multidimensional constellations highly depends on the type of the channel, it is crucial to employ a constellation that is suitable for a certain application. In the first part of this thesis, we highlight and review the key performance indicators (KPIs) of MdCs that should be considered in their design process for various channel scenarios. We also provide a survey on the known MdCs in the context of SCMA systems with their design criteria. The performance of some of those constellations are evaluated for uncoded and LTE turbo-coded SCMA systems under different channel conditions through extensive simulations. We then investigate the effect of the $5 \mathrm{G}$ low density parity check (LDPC) codes on determining KPIs in designing MdCs under various channel scenarios. Since the optimal maximum likelihood (ML) receiver for SCMA is too complex in most applications, one highly popular detection technique is the message passing algorithm (MPA), which exploits the sparsity structure of SCMA. MPA is a near-optimal technique, where its performance improves with increasing the signal-to-noise-ratio (SNR) and the number of iterations. We design novel SCMA SNR-adaptive MdCs which result in substantial performance gains (as much as $2 \mathrm{~dB}$ ) in comparison to the best known SCMA MdCs in the literature, especially in low-to-medium SNR regions when the number of MPA iterations has to be low, and in the presence of 5G-compliant LDPC codes.

Sphere decoding (SD) based detection schemes for SCMA have recently received attention due to their promising features. However, the existing SD-based schemes can only be applied to systems with constellations that possess a certain structure. In the second part of the thesis, we propose an SD-based detection scheme, namely improved SD (ISD), for SCMA that achieves the optimal ML detector for any arbitrary regular or irregular constellations. 
To whom the world revolves around and who calls for human unity, and

To Lady Fatimah Zahra. 


\section{Acknowledgments}

First, I would be eternally grateful to God to nominate me for the big honor to accomplish my Ph.D. under the careful supervision of the best supervisors one could ever imagine; ones that not only changed the direction of my research, but also my life; ones that have been my main source of inspiration and motivation, and without their patience, support and guidance, I could not stand here today: Thank-you Professors Ian Marsland, Halim Yanikomeroglu, and Ramy Gohary.

I would also like to mention the government of Ontario in acknowledgment of the Ontario Trillium scholarship which pledged a wide support to my Ph.D., Huawei Technologies research and development team, specially Dr. Javad Abdoli, Dr. Gamini Senarath, and Dr. Hadi Baligh, Department of Systems and Computer Engineering of Carleton University to give me the opportunity to teach a fourth-year undergraduate course twice, Research Computing Services Team of Carleton University, specially Mr. Ryan Taylor, to allocate resources to run my extensive simulations, and eventually all who helped me differently, particularly, Dr. Ebrahim Bedeer, Dr. Vilas Joshi, Dr. Zeinab Zeinalikhani, and Irem Bor-Yaliniz.

My thanks also extend to my thesis examination board, specially Professors Rui Dinis and Claude D'Amours for their valuable feedback.

Last but not the least, I would like to express my deep gratitude to my parents, Hassan and Zahra, and my siblings, Shahabeddin and Motahareh, whose love, support, and prayers have been with me during the darkest of times, hardest of hours and from thousands of miles away. Special thanks to my love, Ehsan, for his endless encouragement and support in the last year of my Ph.D. which made a big difference. 


\section{Table of Contents}

Abstract

Acknowledgments $\quad$ v

Table of Contents vi vi

List of Tables $\quad$ ix

List of Figures $\quad$ x

$\begin{array}{ll}\text { Nomenclature } & \text { Xv }\end{array}$

1 Introduction 1

1.1 Motivation ........................ 1

1.2 Objectives and Contributions .................. 4

1.3 List of Publications . . . . . . . . . . . . . . . 5

1.3.1 Published Journal Papers — included in the thesis . . . . . . 5

1.3.2 Submitted Journal Papers — included in the thesis . . . . . . 6

1.3.3 Published Journal Papers — not included in the thesis . . . . 6

1.3.4 Published Conference Papers - included in the thesis . . . . . 6

1.3.5 Submitted Conference Papers — included in the thesis . . . . 6

1.3.6 Published Conference Papers — not included in the thesis . . 7

1.4 Organization of the Thesis . . . . . . . . . . . . . . 7

2 Uplink SCMA System $\quad 8$

2.1 Channel Models . . . . . . . . . . . . . . . . . . . . . . . . . . . . . 12 
3 Multidimensional Constellations for Uplink SCMA Systems - A Comparative Study 15

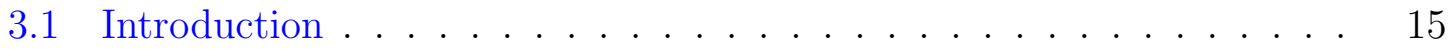

3.2 SCMA Multidimensional Constellation Design . . . . . . . . 16

3.3 Key performance indicators . . . . . . . . . . . . . . . . . . . . 19

3.4 Overview of SCMA Multidimensional Constellations . . . . . . . . . 23

3.5 Performance Evaluation . . . . . . . . . . . . . . . . . 44

3.5.1 Constellations from the Standpoint of KPIs . . . . . . . . 44

3.5 .2 Uncoded Scenarios . . . . . . . . . . . . . . . . . . 46

3.5.3 High-Rate Turbo-coded Scenarios . . . . . . . . . . . . . 53

3.5.4 Low-Rate Turbo-coded Scenarios . . . . . . . . . . . . 59

3.5.5 Summary of Results . . . . . . . . . . . . . . . . 63

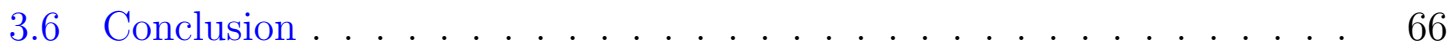

4 Hypercube-based SNR-adaptive Multidimensional Constellation $\begin{array}{ll}\text { Design for Uplink SCMA Systems } & 67\end{array}$

4.1 Introduction . . . . . . . . . . . . . . . . 67

4.2 SCMA MdC Design Problem . . . . . . . . . . . . . . 68

4.2 .1 Rotation-based MdCs . . . . . . . . . . . . . . . . . . . . 69

4.2 .2 Problem Formulation . . . . . . . . . . . . . . . 72

4.3 Proposed Search Method . . . . . . . . . . . . . . . . 80

4.4 Conclusion . . . . . . . . . . . . . . . . . . . . 86

5 SD-based Detection for Generalized SCMA Constellations $\quad 89$

5.1 Introduction . . . . . . . . . . . . . . . . . . . . . . 89

5.2 Detection Problem Formulation . . . . . . . . . . . . . . . . 91

5.2.1 Proposed Detection Scheme . . . . . . . . . . . . . . . 93

5.2 .2 Reduced-complexity ISD . . . . . . . . . . . . . . . 95

5.3 Performance Evaluation _. . . . . . . . . . . . . . 96

5.4 Conclusion . . . . . . . . . . . . . . . . . . . . . . 100

6 Summary and Future Research Directions 101

6.1 Summary . . . . . . . . . . . . . . . . . . . . . . . 101

6.2 Potential Future Research Directions . . . . . . . . . . . . . . . 103

6.2.1 Multidimensional Constellations for SCMA Systems . . . . . . 103 
6.2.2 Dynamic Modulation and Coding . . . . . . . . . . . . . . 104

6.2.3 Joint SCMA Spreading and Constellation Mapping . . . . . . 106

6.2.4 Joint Detection and Decoding for SCMA . . . . . . . . 106

6.2 .5 Grant-free SCMA . . . . . . . . . . . . . . . . 107

$\begin{array}{ll}\text { List of References } & 108\end{array}$

$\begin{array}{ll}\text { Appendix A SCMA Mapping and Spreading } & 120\end{array}$

Appendix B Effects of User-specific Rotations 123

Appendix C Message Passing Algorithm and Bit-LLR Calculation for $\mathrm{BICM}$

Appendix D Uplink SCMA Multidimensional Constellation with LDPC Codes $\quad 128$

C1 Introduction . . . . . . . . . . . . . . . . . . . . 128

C2 High-Rate Scenarios . . . . . . . . . . . . . . . . . . . . . . . . . . 129

C3 Low-Rate Scenarios . . . . . . . . . . . . . . . . . . . . . . . . 132

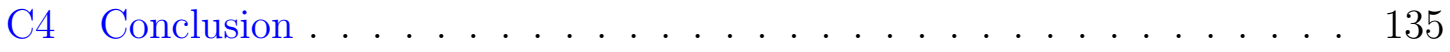




\section{List of Tables}

3.1 An example of a 4-point 2-dimensional complex constellation . . . . . 20

3.2 KPIs of 4-point constellations . . . . . . . . . . . . . . 45

3.3 KPIs of 16-point constellations . . . . . . . . . . . . . . . . . 45

3.4 KPIs of multidimensional constellations for different channel scenarios 65

3.5 Best known multidimensional constellations for different channel scenarios ............................ 65

5.1 Average complexity of ISD and MPA . . . . . . . . . . . 96

A1 An example of a 4-point multidimensional constellation for an SCMA system with $K=6, N=4, d_{v}=2[1] \ldots \ldots$. . . . . . . . . 121

A2 The SCMA codebook with the multidimensional constellation example in Table A1 and the user-to-RE spreading matrix in (2.2) . . . . . . . 122 


\section{List of Figures}

2.1 The uplink SCMA system model with $K$ users. . . . . . . . . . . . . . 9

3.1 Illustration of a sub-optimal process of converting a mother constellation into a user-specific codebook when $d_{v}=4, N=4$, and $M=4$. . 18

3.2 The projection of a 4-point 2-dimensional complex constellation onto the first pre-assigned $\mathrm{RE}\left(\mathrm{RE}_{1}\right)$, and the second pre-assigned $\mathrm{RE}\left(\mathrm{RE}_{2}\right)$. 20

3.3 The projection of 4-point 4-dimensional constellations over $\mathrm{RE}_{1}$ and $\mathrm{RE}_{2}$ : (a) 4-LDS, and (b) T4QAM. . . . . . . . . . . . . . 25

3.4 The projection of 4-point 4-dimensional constellations over $\mathrm{RE}_{1}$ and $\mathrm{RE}_{2}$ : (a) 4LQAM, and (b) 4-Bao. . . . . . . . . . . . . . 26

3.5 The projection of 4-point 4-dimensional constellations over $\mathrm{RE}_{1}$ and $\mathrm{RE}_{2}$ : (a) 4CQAM, and (b) 4-Beko. . . . . . . . . . . . . . 27

3.6 The projection of the 16-LDS constellation over (a) $R_{1}$, and (b) $R E_{2} .28$

3.7 The projection of the T16QAM constellation over (a) $R E_{1}$, and (b) $R E_{2} .30$

3.8 The projection of the 16LQAM constellation over (a) $R E_{1}$, and (b) $R_{2}$. 31

3.9 The projection of the 16-Bao constellation over (a) $R_{1}$, and (b) $R_{2}$. 33

3.10 The projection of the 16HQAM constellation over (a) $\mathrm{RE}_{1}$, and (b) $\mathrm{RE}_{2} 34$

3.11 The projection of the 16CQAM constellation over (a) $R_{1}$, and (b) $R_{2} .36$

3.12 The projection of the $A_{16,5}$ constellation over (a) $\mathrm{RE}_{1}$, and (b) $\mathrm{RE}_{2}$. . 38

3.13 The projection of 16 -Beko over (a) $\mathrm{RE}_{1}$, (b) $\mathrm{RE}_{2}$. . . . . . . . . . 39

3.14 The projection of 4 -Peng over (a) $\mathrm{RE}_{1}$, (b) $\mathrm{RE}_{2} \ldots \ldots$. . . . . . . 41

3.15 The projection of 4-PSCMA over (a) $\mathrm{RE}_{1}$, and (b) $\mathrm{RE}_{2}$. . . . . . . . 42

3.16 The projection of 4-PSCMA over (a) $\mathrm{RE}_{3}$, and (b) $\mathrm{RE}_{4}$. . . . . . . . 43

3.17 SER performance of uncoded SCMA systems with 4-point constellations over FSC (Case 1). . . . . . . . . . . . . . . . . . . 47

3.18 SER performance of uncoded SCMA systems with 16-point constellations over FSC (Case 1). . . . . . . . . . . . . . . . . . . . . 48 
3.19 SER performance of uncoded SCMA systems with 4-point constellations over FIC (Case 2). . . . . . . . . . . . . . . . . . . . . . . . . . 49

3.20 SER performance of uncoded SCMA systems with 16-point constellations over FIC (Case 2). . . . . . . . . . . . . . . .

3.21 BER performance of uncoded SCMA systems with 4-point constellations over FSC (Case 1). . . . . . . . . . . . . . . . 51

3.22 BER performance of uncoded SCMA systems with 16-point constellations over FSC (Case 1). . . . . . . . . . . . . . . . . 51

3.23 BER performance of uncoded SCMA systems with 4-point constellations over FIC (Case 1). . . . . . . . . . . . . . . . . 52

3.24 BER performance of uncoded SCMA systems with 16-point constellations over FIC (Case 1). . . . . . . . . . . . . . .

3.25 FER performance of $R=4 / 5$ turbo-coded SCMA systems with 4-point constellations over FFSC (Case 3).

3.26 FER performance of $R=4 / 5$ turbo-coded SCMA systems with 16point constellations over FFSC (Case 3). . . . . . . . . . . . . . 54

3.27 FER performance of $R=4 / 5$ turbo-coded SCMA systems with 4-point constellations over FFIC (Case 4) . . . . . . . . . . . . . 56

3.28 FER performance of $R=4 / 5$ turbo-coded SCMA systems with 16 point constellations over FFIC (Case 4). . . . . . . . . . . . . 56

3.29 FER performance of $R=4 / 5$ turbo-coded SCMA systems with 4-point constellations over SFSC (Case 5).

3.30 FER performance of $R=4 / 5$ turbo-coded SCMA systems with 16point constellations over SFSC (Case 5).

3.31 FER performance of $R=4 / 5$ turbo-coded SCMA systems with 4-point constellations over SFIC (Case 6) . . . . . . . . . . . . . . . . . 58

3.32 FER performance of $R=4 / 5$ turbo-coded SCMA systems with 16point constellations over SFIC (Case 6). . . . . . . . . . .

3.33 FER performance of $R=1 / 3$ turbo-coded SCMA systems with 4-point constellations over FFSC (Case 3).

3.34 FER performance of $R=1 / 3$ turbo-coded SCMA systems with 16point constellations over FFSC (Case 3).

3.35 FER performance of $R=1 / 3$ turbo-coded SCMA systems with 4-point constellations over FFIC (Case 4) . . . . . . . . . . . . 
3.36 FER performance of $R=1 / 3$ turbo-coded SCMA systems with 16point constellations over FFIC (Case 4). . . . . . . . . . . . 61

3.37 FER performance of $R=1 / 3$ turbo-coded SCMA systems with 4-point constellations over SFSC (Case 5). . . . . . . . . . . . . . . 62

3.38 FER performance of $R=1 / 3$ turbo-coded SCMA systems with 16point constellations over SFSC (Case 5). . . . . . . . . . . .

3.39 FER performance of $R=1 / 3$ turbo-coded SCMA systems with 4-point constellations over SFIC (Case 6). . . . . . . . . . . . . . . . 64

3.40 FER performance of $R=1 / 3$ turbo-coded SCMA systems with 16point constellations over SFIC (Case 6). . . . . . . . . . . . . . . . . 64

4.1 The projections of 16HQAM (brown crosses) and T16QAM (red circles) onto $\mathrm{RE}_{1}$ and $\mathrm{RE}_{2}$. . . . . . . . . . . . . . . . . . . . . . 71

4.2 The effect of changing $\theta$ on FER at $E_{\mathrm{mb}} / N_{0}=17 \mathrm{~dB}$ for different $N_{\mathrm{it}} \mathrm{s} . \quad 76$

4.3 The effect of changing $\theta$ on the FER performance of an LDPC-coded SCMA systems with $N_{\text {it }}=1 \ldots \ldots$. . . . . . . . . 77

4.4 The effect of changing $\theta$ on the FER performance of an LDPC-coded

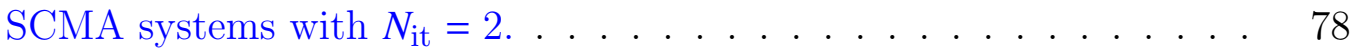

4.5 The effect of changing $\theta$ on the FER performance of an LDPC-coded SCMA systems with $N_{\text {it }}=3 \ldots \ldots$. . . . . . . . . . . . 78

4.6 The effect of changing $\theta$ on the FER performance of an LDPC-coded SCMA systems with $N_{\text {it }}=4 \ldots \ldots$. . . . . . . . . . 79

4.7 The effect of changing $\theta$ on the FER performance of an LDPC-coded SCMA systems with $N_{\text {it }}=5 \ldots \ldots$. . . . . . . . . . . . . 79

$4.8\left\{\operatorname{FER}\left(\theta_{j}\right)\right\}$ versus $\left\{\theta_{j}\right\}$ for $N_{s}=100, N_{s}=7$, and the fitted secondorder rational polynomial to the $N_{s}=7$ case, when $E_{\mathrm{mb}} / N_{0}=17 \mathrm{~dB}$ and $N_{\mathrm{it}} \leq 5 . \ldots \ldots \ldots \ldots \ldots \ldots \ldots$

4.9 The effect of changing $E_{\mathrm{mb}} / N_{0}$ on $\theta_{\text {copt }}$ for different $N_{\text {it }} \leq 5$ using the rational-polynomial fit to $N_{s}=7$. . . . . . . . . . . . . . . . 82

4.10 The effect of changing $N_{\text {it }}$ on $\theta_{\text {copt }}$ for $12 \leq E_{\mathrm{mb}} / N_{0} \leq 20$ using the rational-polynomial fit to $N_{s}=7$.

4.11 FER performance comparison of an SCMA system with the 16HQAM $(\theta=0)$, T16QAM $\left(\theta=\theta_{\mathrm{mx}}\right)$, and $\theta=\theta_{\text {copt }}$ for different $E_{\mathrm{mb}} / N_{0}$ s and $N_{\text {it }}=1 \ldots \ldots \ldots \ldots \ldots \ldots \ldots \ldots \ldots \ldots \ldots \ldots \ldots \ldots \ldots \ldots$ 
4.12 FER performance comparison of an SCMA system with the 16HQAM $(\theta=0)$, T16QAM $\left(\theta=\theta_{\mathrm{mx}}\right)$, and $\theta=\theta_{\mathrm{copt}}$ for different $E_{\mathrm{mb}} / N_{0} \mathrm{~s}$ and $N_{\mathrm{it}}=2 \ldots \ldots \ldots \ldots \ldots \ldots \ldots \ldots$

4.13 FER performance comparison of an SCMA system with the 16HQAM $(\theta=0)$, T16QAM $\left(\theta=\theta_{\mathrm{mx}}\right)$, and $\theta=\theta_{\text {copt }}$ for different $E_{\mathrm{mb}} / N_{0 \mathrm{~s}}$ and $N_{\mathrm{it}}=3 \ldots \ldots \ldots \ldots \ldots \ldots$

4.14 FER performance comparison of an SCMA system with the 16HQAM $(\theta=0)$, T16QAM $\left(\theta=\theta_{\mathrm{mx}}\right)$, and $\theta=\theta_{\text {copt }}$ for different $E_{\mathrm{mb}} / N_{0 \mathrm{~s}}$ and $N_{\mathrm{it}}=4 \ldots \ldots \ldots \ldots \ldots \ldots \ldots \ldots \ldots \ldots \ldots \ldots \ldots \ldots \ldots$

4.15 FER performance comparison of an SCMA system with the 16HQAM $(\theta=0)$, T16QAM $\left(\theta=\theta_{\mathrm{mx}}\right)$, and $\theta=\theta_{\text {copt }}$ for different $E_{\mathrm{mb}} / N_{0} \mathrm{~s}$ and $N_{\text {it }}=5 \ldots \ldots \ldots \ldots \ldots \ldots \ldots \ldots \ldots \ldots$ 86

5.1 An example of the tree branching process. . . . . . . . . . . . . 95

5.2 Performance of uncoded SCMA systems over FSC and FIC. All users employ the 4-Beko constellation. . . . . . . . . . . . . . . .

5.3 Performance of uncoded SCMA systems over FSC and FIC. All users employ the T16QAM constellation. . . . . . . . . . . . . . . . . 97

5.4 Average RAs and RMs in ISD and MPA with the constellation in [1].

5.5 Average RAs and RMs in an uncoded SCMA system with the 4-Beko constellation. . . . . . . . . . . . . . . . .

5.6 Average RAs and RMs in an uncoded SCMA system with the T16QAM constellation.

B1 The illustration of the importance of user-specific rotations in (a) a downlink, and (b) an uplink scenario. . . . . . . . . . . . . . 124

C1 An example of the factor graph for $K=4$ users and $N=6$ resources. . 126

C1 FER performance of LTE turbo-coded and 5G-compliant LDPC-coded systems with $R=4 / 5$ over FFSC (Case 3) . . . . . . . . . . . . . . 129

C2 FER performance of LTE turbo-coded and 5G-compliant LDPC-coded systems with $R=4 / 5$ over FFIC (Case 4). . . . . . . . . . . . . . 130

C3 FER performance of LTE turbo-coded and 5G-compliant LDPC-coded systems with $R=4 / 5$ over SFSC (Case 5). . . . . . . . . .

C4 FER performance of LTE turbo-coded and 5G-compliant LDPC-coded systems with $R=4 / 5$ over SFIC (Case 6). . . . . . . . . . . 131 
C5 FER performance of LTE turbo-coded and 5G-compliant LDPC-coded systems with $R=4 / 5$ over FFSC (Case 3). . . . . . . . . . . . 132

C6 FER performance of LTE turbo-coded and 5G-compliant LDPC-coded systems with $R=4 / 5$ over FFIC (Case 4). . . . . . . . . . . 133

C7 FER performance of LTE turbo-coded and 5G-compliant LDPC-coded systems with $R=4 / 5$ over SFSC (Case 5). . . . . . . . . . 133

C8 FER performance of LTE turbo-coded and 5G-compliant LDPC-coded systems with $R=4 / 5$ over SFIC (Case 6). . . . . . . . . . . 134 


\section{Nomenclature}

\section{List of abbreviations}

AWGN Additive white Gaussian noise

BER Bit error rate

BICM Bit-interleaved coded modulation

CSI Channel state information

FEC Forward error control

FER Frame error rate

FFIC Fast fading with independent channel coefficients

FFSC Fast fading with a single channel coefficient

FIC Fading with independent channel coefficient

FSC Fading with a single channel coefficient

FLOPs Floating-point operations

i.i.d. Independent and identically distributed

IoT Internet of Things

ISD Improved sphere decoding

KPI Key performance indicator

LDS Low density signature/spreading

MCS Modulation and coding scheme

MdCs Multidimensional constellations 
ML Maximum likelihood

MPA Message passing algorithm

MUD Multiuser detection

MUI Multiuser interference

NOMA Non-orthogonal multiple access

RE Resource element

SCMA Sparse code multiple access

SD Sphere decoding

SER Symbol error rate

SIC Successive interference cancellation

SNR Signal-to-noise ratio

SFIC Quasi-static fading with independent channel coefficients

SFSC Quasi-static fading with a single channel coefficient 


\section{Frequently used symbols}

\begin{tabular}{|c|c|}
\hline $\mathbf{a}_{k}$ & Sequence of information bits \\
\hline $\mathbb{B}$ & Set of binary numbers \\
\hline$c$ & $\cos \theta$ \\
\hline$d_{c}$ & Number of users per RE \\
\hline$d_{E, \min }$ & Minimum Euclidean distance \\
\hline$d_{P, \min }$ & Minimum Product distance \\
\hline$d_{v}$ & Number of REs assigned to each user \\
\hline$E_{\mathrm{mb}}$ & Energy per message bit \\
\hline$E_{\mathrm{S}}$ & Energy per symbol bit \\
\hline $\mathbf{F}$ & Binary user-to-RE indicator matrix \\
\hline $\mathbf{G}_{u, v}(\theta)$ & Givens rotation based on $\theta$ along the $u$ th row and $v$ th column \\
\hline G & Effective channel matrix \\
\hline $\mathbf{h}_{k, i}$ & $N$-dimensional channel coefficients of the $k$ th user at the $i$ th channel use \\
\hline$K$ & Number of users \\
\hline$K_{c}$ & Length of information bits \\
\hline$L$ & Modulation diversity order in Chapter 3, and number of layers in Chapter 5 \\
\hline$L_{M}$ & Number of bits in each constellation point \\
\hline$M$ & Constellation size \\
\hline$N$ & Number of orthogonal resource elements (REs) \\
\hline$N_{0}$ & Single-sided noise power spectral density \\
\hline$N_{c}$ & Length of coded bits (output of channel encoder) \\
\hline$N_{c u}$ & Number of channel uses \\
\hline
\end{tabular}


$N_{d} \quad$ Number of distinct points

$N_{\text {it }} \quad$ Number of MPA iterations

$R \quad$ Code rate

$\mathbf{R}(\theta) \quad$ Rotation matrix as a function of $\theta$

$s \quad \sin \theta$

S Binary user-to-RE spreading matrix

$\tau_{E} \quad$ Euclidean kissing number

$\tau_{P} \quad$ Product kissing number

$\theta \quad$ Rotation angle

$\mathcal{V} \quad N$-dimensional sparse codebook of user $k$

$\mathbf{v}_{k, m} \quad m$ th codeword of $N$-dimensional complex codeword of user $k$

$\hat{\mathbf{V}}_{i} \quad$ Estimated codeword for all users at the $i$ th channel use

$\tilde{\mathbf{v}}_{k, i} \quad N$-dimensional transmitted complex codeword of user $k$ at the $i$ th channel use

$\mathbf{w}_{i} \quad$ Vector of i.i.d. complex Gaussian noise

$\mathcal{X}^{+} \quad$ Set of mother constellation points

$\mathbf{x}_{m}^{+} \quad m$ th symbol of mother constellation

$\mathcal{X}_{k} \quad$ Set of constellation points of user $k$

$\mathbf{x}_{k, m} \quad m$ th symbol of constellation of user $k$

$\tilde{\mathbf{x}}_{k, i} \quad$ Transmitted constellation point of user $k$ at the $i$ th channel use

$\mathbf{x} \quad$ Detected symbol for all users

$\mathbf{y}_{i} \quad$ Received vector at the $i$ th channel use 


\section{Chapter 1}

\section{Introduction}

\subsection{Motivation}

One key difference among the generations of wireless systems from $1 \mathrm{G}$ to $4 \mathrm{G}$ arises in various multiple access schemes. In particular, $1 \mathrm{G}$ operates with frequency division multiple access (FDMA), 2G with time division multiple access (TDMA), 3G with code division multiple access (CDMA), and 4G with orthogonal frequency division multiple access (OFDMA). FDMA, TDMA, CDMA, and OFDMA are generally orthogonal multiple access (OMA) schemes, wherein orthogonal resource elements (REs) are allocated to different users to avoid the interference among them [2,3]. For example, a RE could be one narrow frequency band used over one short time slot, or it could also be divided by orthogonal codewords ${ }^{1}$. To provide even higher spectral efficiency, future wireless systems are likely to employ non-orthogonal multiple access (NOMA) [4-10]. NOMA allocates REs to users in a non-orthogonal fashion, allowing multiple users to share the same REs. It improves the spectral efficiency of the system, meeting the ever-growing demand for mobile Internet and the Internet-of-Things (IoT) [11-13].

The various NOMA techniques can be categorized into three main classes [14]: multiple-domain NOMA, power-domain NOMA, and code-domain NOMA.

\footnotetext{
${ }^{1}$ To be precise, in 3G, the downlink uses orthogonal CDMA (Walsh-Hadamard codes); but the uplink in general uses non-orthogonal CDMA through pseudo noise-codes (PN-codes). This is due to the fact that orthogonality requires chip-level synchronization; this is next-to-trivial to achieve in the downlink, while very difficult in the uplink. That is, even if the orthogonal Walsh-Hadamard codes are used in the uplink, since the chip-level synchronization is lost due to different propagation times, the received signals at the BS become no longer chip-level synchronized, i.e., the orthogonality is not maintained. As such, other design alternatives that perform better in the absence of chip-level synchronization are used instead of the Walsh-Hadamard codes.
} 
Power-domain NOMA (also referred as single-carrier NOMA [15]) can support multiple users within the same RE by allocating different power levels to them based on their channel conditions [16-18]. Successive interference cancellation (SIC) is used at the receiver to detect the transmitted signals. It is shown in [19] that if both NOMA and OMA employ optimal resource allocation, NOMA can still outperform the conventional OMA. Power-domain NOMA is considered as a potential scheme for $5 \mathrm{G}$ and beyond systems, especially for downlink scenarios [14,20].

Code-domain NOMA, also referred to as multi-carrier NOMA in [15], can serve multiple users within the same RE. In code-domain NOMA, users are differentiated by their different codes. Low density signature/spreading (LDS) [21-26] is one of the early code-domain NOMA techniques, where each user's data is spread over multiple REs in a sparse manner. In other words, each user is assigned to only a subset of the available REs. As such, the multiuser interference pattern at the receiver entails a low density graph [27], reducing the complexity of the multiuser detection at the receiver. In LDS, all users employ the repetition of quadrature amplitude modulation (QAM) constellations to spread their information over their pre-assigned REs. At the receiver, due to the sparseness of LDS, the widely used message passing algorithm (MPA) [28] can be used for detection.

Sparse code multiple access (SCMA) [29] is a novel code-domain NOMA scheme that is essentially an enhanced variation of LDS. Like with LDS, each RE is shared among only a subset of the active users, constituting a sparse structure, but SCMA users map their incoming bits to multidimensional constellations (MdCs) to spread their information instead of using the repetition of QAM over their pre-assigned REs. For instance, if each user is assigned to two REs, LDS transmits the repetition of one QAM symbol over both REs, whereas SCMA transmits a different symbol over each RE. Each SCMA transmission is a component of an MdC. In [29,30], LDS is compared with SCMA from different aspects; LDS is considered as a special class of SCMA, where the MdC is just the repetition of QAM.

Multiple-domain NOMA superimposes multiple users in multiple domain, such as the power domain, the code domain, and the spatial domain [14]. Pattern division multiple access (PDMA) [31] and lattice partition multiple access (LPMA) [32] are two examples of this type of NOMA. In PDMA, similar to SCMA, the users spread their data over their pre-assigned REs in a sparse manner. However, unlike SCMA, the number of REs assigned to each PDMA user can vary. Further, PDMA users can 
be multiplexed in power or space domains as well. When different clusters of users are multiplexed in different domains, MPA followed by SIC is employed for detection [14]. In LPMA, power and code domains are combined to multiplex users. A multilevel lattice code assigns different lattice codes to different users based on their channel conditions. At the receiver and similar to power-domain NOMA, SIC can be used for detection.

The comparison among different NOMA techniques has been widely studied from different aspects in e.g., [3,14,33-36]. It is found in [14] that the structure of powerdomain NOMA is uncomplicated, and thus, it can be combined with other different technologies like multiple-input multiple output (MIMO) or cooperative networks. On the other hand, to order the users based on their channel conditions, clustering and pairing of users are required, which will add to the complexity of the system. Codedomain NOMA and multiple-domain NOMA do not require the channel conditions of different users. Moreover, the near-optimal MPA detection used in code-domain and multiple-domain NOMA can perform better than SIC used in power-domain NOMA, but MPA is more complex. In addition, the coding that is being used in code-domain NOMA and some multiple-domain NOMA techniques suggests some redundancies, which reduces the spectral efficiency of the system. In [33], it is shown that compared with PDMA, SCMA achieves a better performance due to its multidimensional constellation. In [34], the power-domain NOMA and SCMA are compared from the sum-rate perspective. The numerical analysis in [34] shows that SCMA achieves a better sum-rate compared with power-domain NOMA, but at the cost of a higher system complexity. A comprehensive study of different NOMA schemes is carried out in [35]. It is found in [35] that the code-domain NOMA can achieve a higher robustness and is a better candidate for massive connectivity. In [3], a systematic overview on the design of different NOMA schemes along with the related standardization process for the next generation of wireless networks are provided. Furthermore, SCMA is presented as a potential uplink NOMA scheme that is proposed in 3GPP Release-14 for the system design of the new radio (NR) [3], and as a low latency multiple access solution for future systems [36].

Due to the attractive features of code-domain NOMA, and particulary SCMA, it is important to investigate SCMA from different aspects. One of the most appealing applications of SCMA systems is in uplink. In particular, SCMA has been proposed to support uplink machine-type communication services $[2,3,37]$. As such, the focus 
of this thesis is on uplink SCMA systems.

\subsection{Objectives and Contributions}

The objective of this thesis is to investigate uplink SCMA systems from two main aspects:

Multidimensional Constellations: In an SCMA system, designing MdCs plays an important role in the performance of the system. In fact, it is a key feature that distinguishes SCMA from other NOMA techniques [38]. Since the behavior of MdCs highly depends on the type of the channel, it is crucial to employ a constellation that is suitable for a certain application.

In Chapter 3, we highlight and identify the key performance indicators (KPIs) of MdCs that should be considered in their design process for various channel scenarios. We also provide a survey on the known MdCs in the context of SCMA systems with their design criteria. We then evaluate the performance of some of those constellations for uncoded, high-rate, and low-rate LTE turbocoded [39] SCMA systems under different channel conditions through extensive simulations. This work is the most comprehensive study by far on designing MdCs for SCMA system.

Since the optimal maximum likelihood (ML) receiver for SCMA is too complex in most applications, one highly popular detection technique is MPA, which exploits the sparsity structure of SCMA. MPA is a near-optimal technique, where its performance improves with increasing the signal-to-noise-ratio (SNR) and the number of iterations. It is known that the MPA performance asymptotically approaches the ML performance when the number of iterations goes to infinity. When the number of MPA iterations has to be limited (such as low-latency and/or low-complexity and/or energy-sensitive applications), the performance gap between ML and MPA becomes significant, especially in lowto-medium SNR regions. Also, it is known that the Gray-labelled MdCs that are constructed based on hypercubes perform well when used along with bitinterleaved coded modulation as they obtain an equal error protection among their different bit levels. In Chapter 4, we design novel SCMA SNR-adaptive 
MdCs which result in substantial performance gains (as much as $2 \mathrm{~dB}$ ) in comparison to the best known SCMA MdCs in the literature, especially in low-tomedium SNR regions when the number of MPA iterations has to be low, and in the presence of 5G-compliant LDPC codes [40]. The proposed novel MdCs are obtained by optimized unitary rotations on hypercubes and by exploiting the attractive features of them, which result in limiting the search for finding the optimal rotations.

Multiuser Detection: One of the key aspects in the design of SCMA systems is to deploy efficient multiuser detection schemes that recovers the transmitted data from each active user [41]. Sphere decoding (SD) based detection schemes for SCMA systems have recently received attention due to their promising features. However, the existing SD-based schemes can only be applied on SCMA systems with constellations possessing a certain structure.

In Chapter 5, we propose a novel SD-based detection scheme, namely improved SD (ISD), for SCMA that achieves the optimal ML detector for any arbitrary regular or irregular constellations. To overcome the rank deficiency problem of the SCMA channel matrix, we fix a portion of the transmitted symbols and obtain an optimal detection problem that is equivalent to the original SCMA detection problem for all types of constellations. Moreover, due to the sparse nature of SCMA, the partial metric at each layer is evaluated in such a way that is independent of users assigned to each resource element. This, in turn, reduces the average complexity of ISD.

\subsection{List of Publications}

Thus far, our main contributions in this thesis, which are published or submitted for publication, are as follows:

\subsubsection{Published Journal Papers — included in the thesis}

[J1] Monirosharieh Vameghestahbanati, Ebrahim Bedeer, Ian Marsland, Ramy Gohary, and Halim Yanikomeroglu, "Enabling sphere decoding for SCMA", IEEE Commun. Lett., vol. 21, no. 12, pp. 2750-2753, Dec. 2017. 
[J2] Monirosharieh Vameghestahbanati, Ian Marsland, Ramy Gohary, and Halim Yanikomeroglu, "Multidimensional constellations for uplink SCMA systems - A comparative study", IEEE Commun. Surveys Tuts., vol. 21, no. 3, pp. 21692194, Third Quart., 2019.

[J3] Monirosharieh Vameghestahbanati, Ian Marsland, Ramy Gohary, and Halim Yanikomeroglu, "A novel SD-based detection for generalized SCMA constellations", IEEE Trans. Veh. Technol., vol. 68, no. 10, pp. 10278-10282, Oct. 2019.

\subsubsection{Submitted Journal Papers — included in the thesis}

[J4] Monirosharieh Vameghestahbanati, Ian Marsland, Ramy Gohary, and Halim Yanikomeroglu, "Hypercube-based SNR-adaptive multidimensional constellation design for uplink SCMA systems", under review in IEEE Trans. Commun. (submission: 07 Nov. 2019).

\subsubsection{Published Journal Papers — not included in the thesis}

[J5] Aditya S. Rajasekaran, Monirosharieh Vameghestahbanati, Mohammad Farsi, Halim Yanikomeroglu, and Hamid Saeedi, "Resource allocation based PAPR analysis in uplink SCMA-OFDM systems", IEEE Access, vol. 7, pp. 162803-162817, Dec. 2019.

\subsubsection{Published Conference Papers — included in the thesis}

[C1] Monirosharieh Vameghestahbanati, Ian Marsland, Ramy Gohary, and Halim Yanikomeroglu: "Key performance indicators in multidimensional constellations for uplink SCMA systems", in Proc. Canadian Workshop on Information Theory (CWIT), 02-05 Jun. 2019, Hamilton, Canada.

\subsubsection{Submitted Conference Papers — included in the thesis}

[C2] Monirosharieh Vameghestahbanati, Ian Marsland, Ramy Gohary, Halim Yanikomeroglu, and Javad Abdoli: "How does channel coding affect the design of uplink SCMA multidimensional constellations?", under review in Wireless 
Communications and Networking Conference (WCNC), 06-09 Apr. 2020, Seoul, South Korea.

\subsubsection{Published Conference Papers - not included in the thesis}

[C3] Monirosharieh Vameghestahbanati, Ian Marsland, Ramy Gohary, and Halim Yanikomeroglu: "Polar codes for SCMA systems", in Proc. IEEE Vehicular Technology Conference (VTC2017-Fall), 24-27 Sep. 2017, Toronto, Canada.

\subsection{Organization of the Thesis}

The rest of this thesis is organized as follows. We describe the detailed uplink SCMA system model in Chapter 2. We then demonstrate the main contributions of this thesis through two main parts: SCMA MdCs (Chapter 3-Chapter 4), and SCMA multiuser detection (Chapter 5). In Chapter 6, we summarize the thesis and shed some light for future research directions. 


\section{Chapter 2}

\section{Uplink SCMA System}

Consider an uplink SCMA system with $K$ users and $N$ orthogonal resource elements (REs), where $N<K$ (a system is overloaded if $N<K$ ). For example, a RE could be one narrow frequency band used over one short time slot. Each user is assigned to only $d_{v} \ll N$ REs out of the $N$ REs. In other words, each user spreads its data over $d_{v}$ REs. Under the constraint that no two users should be assigned all the same REs, a system is fully overloaded if $K=\left(\begin{array}{l}N \\ d_{v}\end{array}\right)$.

The allocation of the $d_{v}$ REs to the $k$ th user, $k \in\{1, \ldots, K\}$, is indicated through the $N \times d_{v}$ binary user-to-RE spreading matrix, $\mathbf{S}_{k}$. Each row of $\mathbf{S}_{k}$ corresponds to one of the REs. Due to the sparse structure of SCMA, there is only a single non-zero element in each column of $\mathbf{S}_{k}$, with its position determined by the row corresponding to one of the REs assigned to user $k$. As an example, with $d_{v}=2$ and $N=4$, if the first user were assigned to the second and fourth (out of $N=4$ ) REs, then $\mathbf{S}_{1}$ would be

$$
\mathbf{S}_{1}=\left[\begin{array}{ll}
0 & 0 \\
1 & 0 \\
0 & 0 \\
0 & 1
\end{array}\right] .
$$

The full binary user-to-RE spreading matrix of an SCMA system is an $N \times K d_{v}$ matrix, denoted by $\mathbf{S}=\left[\mathbf{S}_{1} \ldots \mathbf{S}_{K}\right]$. Each $d_{v}$ columns of $\mathbf{S}$ corresponds to each user. More precisely, the first $d_{v}$ columns of $\mathbf{S}$, i.e., columns 1 through $d_{v}$, constitutes $\mathbf{S}_{1}$, the second $d_{v}$ columns, i.e., $d_{v}+1$ through $2 d_{v}$, corresponds to $\mathbf{S}_{2}$, and in general 


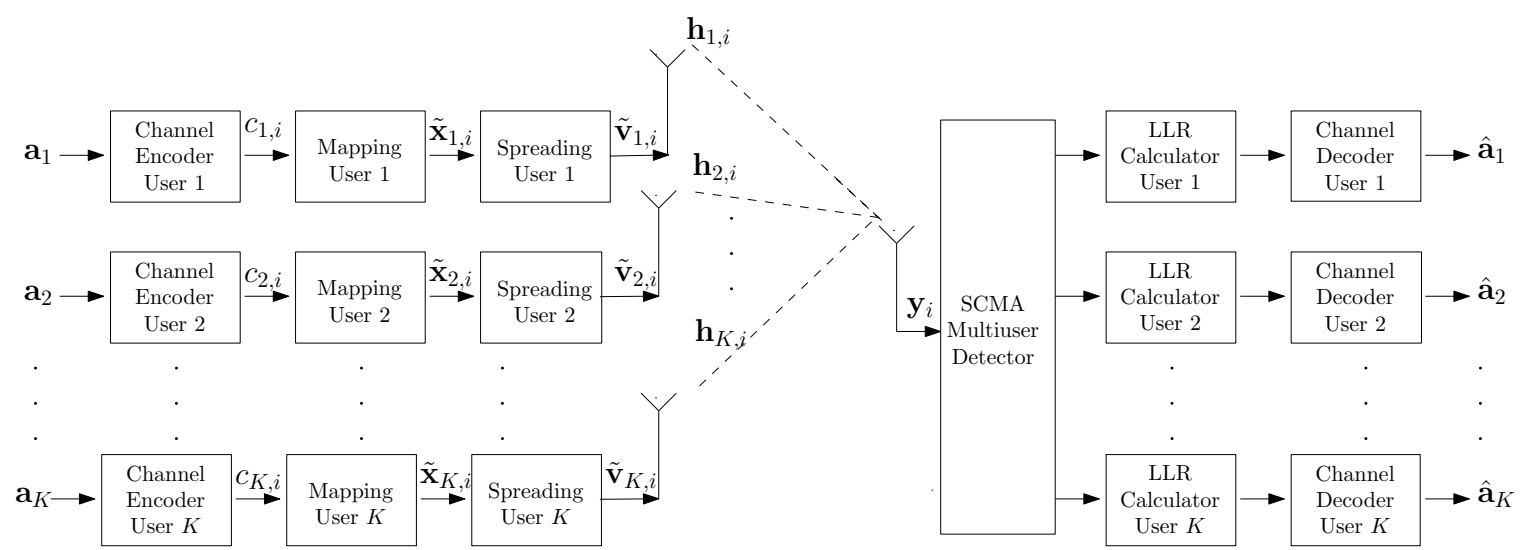

Figure 2.1: The uplink SCMA system model with $K$ users.

the $k$ th $d_{v}$ columns of $\mathbf{S}$, i.e., columns $(k-1) d_{v}+1$ through $k d_{v}$, constitutes $\mathbf{S}_{k}$. To demonstrate, for a fully-loaded SCMA system with $K=6, N=4$ and $d_{v}=2$, the $4 \times 12$ user-to-RE spreading matrix can be

$$
\mathbf{S}=\left[\begin{array}{llllllllllll}
0 & 0 & 1 & 0 & 1 & 0 & 0 & 0 & 1 & 0 & 0 & 0 \\
1 & 0 & 0 & 0 & 0 & 1 & 0 & 0 & 0 & 0 & 1 & 0 \\
0 & 0 & 0 & 1 & 0 & 0 & 1 & 0 & 0 & 0 & 0 & 1 \\
0 & 1 & 0 & 0 & 0 & 0 & 0 & 1 & 0 & 1 & 0 & 0
\end{array}\right]
$$

A concise representation of the allocation of the $d_{v}$ REs to the $k$ th user is performed through the $N$-dimensional binary user-to-RE indicator column vector that is defined by $\mathbf{f}_{k}=\operatorname{diag}\left(\mathbf{S}_{k} \mathbf{S}_{k}^{\mathrm{T}}\right)$, where a value of 1 in the $n^{\text {th }}$ elements of $\mathbf{f}_{k}$ indicates that the $n$th RE has been assigned to the $k$ th user. The user-to-RE indicator matrix, $\mathbf{F}=\left[\mathbf{f}_{1} \ldots \mathbf{f}_{K}\right]$ constitutes an $N \times K$ matrix with each of its columns corresponding to each user with $d_{v}$ non-zero elements, identifying the $d_{v}$ REs that are assigned to each user. In a similar vein, each row of $\mathbf{F}$ corresponds to each RE with $d_{c}$ non-zero elements, identifying the users assigned to each RE. For the previous example in (2.2), 
the user-to-RE indicator matrix is given as

$$
\mathbf{F}=\left[\begin{array}{llllll}
0 & 1 & 1 & 0 & 1 & 0 \\
1 & 0 & 1 & 0 & 0 & 1 \\
0 & 1 & 0 & 1 & 0 & 1 \\
1 & 0 & 0 & 1 & 1 & 0
\end{array}\right]
$$

The first column of $\mathbf{F}$ corresponds to the first user, which is assigned to the second and the fourth REs; the second column of $\mathbf{F}$ corresponds to the second user that is connected to the first and the third REs, and so on. Furthermore, the first row of $\mathbf{F}$ corresponds to the first RE, which is used by the second, third, and fifth users.

The uplink SCMA system model is shown in Fig. 2.1. Let $\mathbf{a}_{k} \in \mathbb{B}^{K_{c}}(\mathbb{B}=\{0,1\}$ is the set of binary numbers) denote the message word of user $k$ that contains a sequence of independent information bits with a length of $K_{c}$. The message word of each user is fed to its corresponding channel encoder to produce a codeword of length $N_{c}$. In an $M$-point signal constellation of each user, each $L_{M}=\log _{2} M$ bits are represented by one constellation point. The codewords from the channel encoders are then divided into $N_{c u}=N_{c} / L_{M}$ digital symbols of $L_{M}$ bits each. In essence, $N_{c u}$ represents the number of channel uses required to transmit one codeword. Let $c_{k, i} \in \mathbb{B}^{L_{M}}, k \in\{1, \ldots, K\}, i \in\left\{1, \ldots, N_{c u}\right\}$ denote the $i$ th digital symbol of user $k$. Each symbol of the $k$ th user is then mapped to a $d_{v}$-dimensional complex constellation point, $\tilde{\mathbf{x}}_{k, i}=\left(\tilde{x}_{1, k, i}, \ldots, \tilde{x}_{d_{v}, k, i}\right)^{\mathrm{T}}$, which is equivalent to a $2 d_{v}$-dimensional real constellation point, and is selected from a $d_{v}$-dimensional complex constellation ${ }^{1}$, $\mathcal{X}_{k}=\left\{\mathbf{x}_{k, m} \mid m=1, \ldots, M\right\}$ of size $M$, where each element is a $d_{v}$-dimensional complex vector, $\mathbf{x}_{k, m}=\left(x_{1, k, m}, \ldots, x_{d_{v}, k, m}\right)^{\mathrm{T}}$.

In an SCMA spreading process, each $d_{v}$-dimensional complex constellation point, $\tilde{\mathbf{x}}_{k, i} \in \mathcal{X}_{k}$, is $\operatorname{coded}^{2}$ to an sparse $N$-dimensional complex codeword, $\tilde{\mathbf{v}}_{k, i}$, through the

\footnotetext{
${ }^{1}$ From now on, the terms " $d_{v}$-dimensional complex constellation" and "multidimensional constellation" are used interchangeably.

${ }^{2}$ To clarify, an SCMA multidimensional constellation point is a $d_{v}$-dimensional complex constellation point without the effect of the user-to-RE spreading matrix. An SCMA codeword is an SCMA multidimensional constellation point with the effect of the user-to-RE spreading matrix. Moreover, in an SCMA system with the presence of channel coding (coded SCMA), there are two notions of codewords; one is the binary codeword that is the output of the channel encoder, and the other is the complex codeword which corresponds to the output of the SCMA spreading. The set of the complex codewords is called a codebook.
} 
binary user-to-RE spreading matrix, $\mathbf{S}_{k}$. That is, $\tilde{\mathbf{v}}_{k, i}=\mathbf{S}_{k} \tilde{\mathbf{x}}_{k, i}$. The $N$-dimensional complex codeword $\tilde{\mathbf{v}}_{k, i}$ is a sparse vector with $d_{v} \ll N$ non-zero entries, and is selected from an $N$-dimensional sparse codebook, $\mathcal{V}_{k}=\left\{\mathbf{v}_{k, m}=\mathbf{S}_{k} \mathbf{x}_{k, m} \mid m=1, \ldots, M\right\}$ of size $M$. In other words, each $d_{v}$-dimensional element of $\mathcal{X}_{k}$ is spread over its corresponding $N$-dimensional element of $\mathcal{V}_{k}$. Each element of $\mathcal{V}_{k}$ constitutes one $N$-dimensional SCMA sparse codeword, $\mathbf{v}_{k, m}=\left(v_{1, k, m}, \ldots, v_{N, k, m}\right)^{\mathrm{T}}$ with $d_{v} \ll N$ non-zero entries. The SCMA mapping and spreading are illustrated through an example in Appendix A.

As shown in Fig. 2.1, the resultant codeword for the $k$ th user, $\tilde{\mathbf{v}}_{k, i}$, is then sent over its corresponding channel. The Rayleigh fading channel coefficient vector for the $k$ th user over the $i$ th channel use is denoted by $\mathbf{h}_{k, i}=\left(h_{1, k, i}, \ldots, h_{N, k, i}\right)^{\mathrm{T}}$, where $h_{n, k, i} \sim \mathcal{C N}(0,1), n \in\{1, \ldots, N\}$. That is, each channel coefficient, $h_{n, k, i}$, has a complex Gaussian distribution with a mean of 0 and a variance of $E\left[\left|h_{n, k, i}\right|^{2}\right]=1$. Note that since each SCMA user spreads its data over $d_{v}$ REs out of the $N$ available REs, only $d_{v}$ of the channel coefficients in $\mathbf{h}_{k, i}$ are relevant. The positions of those $d_{v}$ channel ceofficeints is according to the positions of the non-zero elements in $\mathbf{S}_{k}$, and the correlation between them depends on the channel model under study as we will discuss in Section 2.1.

At the receiver in an uplink SCMA system, the $N$-dimensional received column vector corresponding to the $i$ th channel use, $\mathbf{y}_{i}$, can be expressed as

$$
\begin{aligned}
\mathbf{y}_{i} & =\sum_{k=1}^{K} \operatorname{diag}\left(\mathbf{h}_{k, i}\right) \tilde{\mathbf{v}}_{k, i}+\mathbf{w}_{i} \\
& =\sum_{k=1}^{K} \operatorname{diag}\left(\mathbf{h}_{k, i}\right) \mathbf{S}_{k} \tilde{\mathbf{x}}_{k, i}+\mathbf{w}_{i},
\end{aligned}
$$

where $\mathbf{w}_{i} \sim \mathcal{C N}\left(0, N_{0} \mathbf{I}\right)$ is an $N$-dimensional vector of independent and identically distributed complex Gaussian noise with zero mean and a covariance matrix of $N_{0} \mathbf{I}$.

Given the received vector for each channel use, $\mathbf{y}_{i}$, and the channel fading coefficients for all the users, $\left\{\mathbf{h}_{k, i}\right\}_{k=1}^{K}$, the joint optimal maximum a posteriori (MAP) detection of all the users codewords, $\tilde{\mathbf{V}}_{i}=\left(\tilde{\mathbf{v}}_{1, i}, \ldots, \tilde{\mathbf{v}}_{K, i}\right)$, can be written as

$$
\hat{\mathbf{V}}_{i}=\arg \underset{\tilde{\mathbf{V}}_{i} \in \mathcal{V}}{\max } \operatorname{Pr}\left(\tilde{\mathbf{V}}_{i} \mid \mathbf{y}_{i}\right),
$$


where $\mathcal{V}=\mathcal{V}_{1} \times \mathcal{V}_{2} \times \cdots \times \mathcal{V}_{K}$, and $\operatorname{Pr}\left(\tilde{\mathbf{V}}_{i} \mid \mathbf{y}_{i}\right)$ denotes the a posteriori probability distribution of $\tilde{\mathbf{V}}_{i}$ being transmitted given that $\mathbf{y}_{i}$ was received. The complexity of evaluating (2.5) is high and is proportional to $M^{K}$. In other words, it grows exponentially with the number of users. By exploiting the sparsity of SCMA codewords, the near-optimal MPA detector is widely used instead to evaluate (2.5) iteratively [29]. The complexity of MPA grows exponentially only with the number of users occupying each RE, i.e., it is proportional to $M^{d_{c}}$. The estimated SCMA codewords are then used to calculate the bit log-likelihood-ratios (LLRs), which are fed to each channel decoder to estimate the message word of each user, $\hat{\mathbf{a}}_{k}$. The MPA detector for SCMA with the bit LLR calculations are described in detail in Appendix C.

\subsection{Channel Models}

In uplink SCMA systems, where each user spreads its data over $d_{v}$ REs, the channel can be categorized based on i) the correlation between the fading coefficients of the different REs at each user, and ii) the temporal correlation of the fading coefficients at each RE at different times. In the first category, at one extreme we have fully correlated coefficients (that is, all the coefficients for a user are the same, so no diversity is attained), which typically occurs when the REs are adjacent, and at the other extreme, we have fully independent coefficient, which typically occurs when the REs are far apart. In the second category, which is particularly relevant to systems that use channel coding, at one extreme we have "fast" fading, where fully independent channel coefficients are observed with each channel use, and at the other extreme we have slow "quasi-static" fading, where the channel coefficients remain constant for the duration of the transmission of an entire codeword.

From (2.4) the channel coefficient for the $k$ th user over the $n$th RE and for the $i$ th channel use is denoted by $h_{n, k, i}$. The channel models under study are then as follows:

Case 1: Uncoded fading where each user observes the same channel coefficients over its REs (FSC). More precisely, since in uncoded systems there is only one use of the channel, i.e., $N_{c u}=1, E\left[h_{n, k, 1} h^{*}{ }_{n^{\prime}, k, 1}\right]=1$, for $n, n^{\prime} \in\{1, \ldots, N\}$.

Case 2: Uncoded fading where each user observes independent channel coefficients over its REs (FIC). More precisely, $E\left[h_{n, k, 1} h^{*}{ }_{n^{\prime}, k, 1}\right]=\delta\left[n-n^{\prime}\right]$, where $\delta[\cdot]$ denotes the Kronecker delta function. 
Case 3: Coded fast fading where each user observes the same channel coefficients over its REs (FFSC). More precisely, $E\left[h_{n, k, i} h^{*}{ }_{n^{\prime}, k, i^{\prime}}\right]=\delta\left[i-i^{\prime}\right], i, i^{\prime} \in\left\{1, \ldots, N_{c u}\right\}$.

Case 4: Coded fast fading where each user observes independent channel coefficients over its REs (FFIC). More precisely, $E\left[h_{n^{\prime}, k, i} h^{*}{ }_{n^{\prime}, k, i^{\prime}}\right]=\delta\left[n-n^{\prime}\right] \delta\left[i-i^{\prime}\right]$.

Case 5: Coded quasi-static fading where each user observes the same channel coefficients over its REs (SFSC). More precisely, $E\left[h_{n, k, i} h^{*}{ }_{n^{\prime}, k, i^{\prime}}\right]=1$.

Case 6: Coded quasi-static fading where each user observes independent channel coefficients over its REs (SFIC). More precisely, $E\left[h_{n, k, i} h^{*}{ }_{n^{\prime}, k, i^{\prime}}\right]=\delta\left[n-n^{\prime}\right]$. 


\section{SCMA Multidimensional Constellations}




\section{Chapter 3}

\section{Multidimensional Constellations for Uplink SCMA Systems - A Comparative Study}

\subsection{Introduction}

As SCMA users map their incoming bits to multidimensional constellations, designing good multidimensional constellations plays an important role in their performance. In fact, it is a key feature that distinguishes SCMA from other NOMA techniques [38]. The design of multidimensional constellation has been studied for downlink SCMA systems in e.g., [42-50], and for uplink SCMA systems in e.g., [30,51-64]. The objective of this work is to provide a thorough study on multidimensional constellations for uplink SCMA systems, and to highlight their design metrics under various channel scenarios. The main contributions of the chapter can be summarized as follows:

- We highlight and review the key performance indicators (KPIs) of multidimensional constellations that should be considered in their design process under various channel scenarios.

- We provide an overview of the multidimensional constellations proposed for uplink SCMA systems in [30,51-64] with their design criteria.

- The performance of those constellations are evaluated for uncoded, high-rate, and low-rate LTE turbo-coded SCMA systems over different channel conditions through extensive simulations. 
It is assumed that the channel state information (CSI) is not available at the transmitter. We use the widely used near-optimal message passing algorithm in the logarithmic domain (Log-MPA) (described in Appendix C) for the multiuser detection, and perform the multiuser detection and the turbo decoding separately in a concatenated manner as in [29,30]. All turbo-coded comparisons are performed for bit-interleaved coded modulation (BICM). Simulation results confirm that multidimensional constellations that satisfy KPIs of a certain channel scenario outperform other multidimensional constellations in that scenario. Moreover, the bit error rate (BER) performance of uncoded systems, and the performance of the coded systems are tied to their bit-labeling. The performance of the systems also depends on the behavior of the multi-user detector at different signal-to-noise ratio regions.

\subsection{SCMA Multidimensional Constellation Design}

The structure of an SCMA system, $\mathcal{T}$, with $K$ users and $N$ REs, with the user-to-RE spreading matrix of each user, $\mathbf{S}_{k}$, where each user occupies $d_{v}$ REs, and employs an $M$-point $N$-dimensional codebook, can be written as [30]

$$
\mathcal{T}\left(\mathrm{S}, \mathcal{X} ; K, M, d_{v}, N\right)
$$

where $\mathcal{X}=\mathcal{X}_{1} \times \mathcal{X}_{1} \times \cdots \times \mathcal{X}_{K}$, and $\mathbf{S}=\left[\mathbf{S}_{1} \ldots \mathbf{S}_{K}\right]$. The SCMA codebook design problem involves finding the optimum user-to-RE spreading matrix, $\mathbf{S}^{*}$, along with the optimum multidimensional constellation, $\boldsymbol{X}^{*}$, and can be defined as

$$
\mathbf{S}^{*}, \mathcal{X}^{*}=\arg \max _{\mathbf{S}, \mathcal{X}} D\left(\mathcal{T}\left(\mathbf{S}, \mathcal{X} ; K, M, d_{v}, N\right)\right)
$$

where $D$ is a design criterion. A sub-optimal multi-stage optimization approach is deployed in [30,51-64] for uplink SCMA systems that involves finding $\mathbf{S}^{*}$ first and then $\mathcal{X}^{*}$. Moreover, when the system is fully overloaded, there is a unique solution for $\mathbf{S}^{*}$. As such, the optimization problem of an SCMA system is reduced to

$$
\mathcal{X}^{*}=\arg \max _{\mathcal{X}} D\left(\mathcal{T}\left(\mathbf{S}^{*}, \mathcal{X} ; K, M, d_{v}, N\right)\right)
$$

The problem is then to find $K$ different $M$-point $d_{v}$-dimensional complex constellations. To further simplify this optimization problem, the SCMA multidimensional 
constellation design is often performed in two steps [29]: Firstly, to design a $d_{v^{-}}$ dimensional mother constellation of size $M, X^{+}$, where each element of $\mathcal{X}^{+}$represents one constellation point of the mother constellation, i.e., $\mathbf{x}_{m}^{+}=\left(x_{1, m}^{+}, \ldots, x_{d_{v}, m}^{+}\right)^{\mathrm{T}}$, $m \in\{1, \ldots, M\}$. Secondly, to perform some user-specific unitary rotations on the mother constellation to generate user-specific multidimensional constellations (e.g., [65]). That is,

$$
\mathcal{X}_{k}=\left\{\boldsymbol{\Delta}_{k} \mathbf{x}_{m}^{+} \mid \mathbf{x}_{m}^{+} \in \mathcal{X}^{+}\right\}
$$

where $\boldsymbol{\Delta}_{k}$ represents a $d_{v} \times d_{v}$ user-specific rotation matrix. Therefore, $\mathcal{X}=\left\{\boldsymbol{\Delta}_{k} \mathbf{x}_{m}^{+}\right\}$. The optimization problem in (3.3) then becomes

$$
\left\{\boldsymbol{\Delta}_{k}^{*}\right\}, \mathcal{X}^{+^{*}}=\arg \max _{\left\{\boldsymbol{\Delta}_{k}\right\}, \mathcal{X}^{+}} D\left(\mathcal{T}\left(\mathbf{S}^{*},\left\{\boldsymbol{\Delta}_{k} \mathbf{x}_{m}^{+}\right\} ; K, M, d_{v}, N\right)\right) .
$$

As a suboptimal approach, the mother constellation and the user-specific rotations are typically found separately [30]. More particularly, an $M$-point $d_{v^{-} \text {-dimensional }}$ complex mother constellation, $\boldsymbol{X}^{+}$, is first designed according to the criteria that will be discussed in detail in Section 2.1. The $d_{v} \times d_{v}$ specific rotation for user $k$, $\boldsymbol{\Delta}_{k}$, is applied on the complex $\mathcal{X}^{+}$to create $\mathcal{X}_{k}$. The allocation of REs to the $k$ th user is performed through $\mathbf{S}_{k}$, which is then applied on the $d_{v}$-dimensional complex constellation, $\mathcal{X}_{k}$, to create the codebook $\mathcal{V}_{k}$.

Fig. 3.1 illustrates a sub-optimal process of converting a mother constellation to an SCMA codebook for user $k$ with $N=4, d_{v}=2, M=4$, and $\mathbf{S}_{k}$ for $k=1$ given in (2.1). Each element of $\mathcal{X}^{+}$, i.e., $\mathbf{x}_{m}^{+}$, in Fig. 3.1 is represented by a rectangle consisting of 2 squares; each square is a representation of $x_{j, m}^{+}, j \in\left\{1, \ldots, d_{v}\right\}, d_{v}=2$. The $2 \times 2$ specific rotation for user $k$, i.e., $\boldsymbol{\Delta}_{k}$, is applied to the element of $\mathcal{X}^{+}$to create the 4-point 2-dimensional complex constellation for user $k$, i.e., $\boldsymbol{X}_{k}$. The elements of $\mathcal{X}_{k}$ correspond to $\mathbf{x}_{k, 1}, \mathbf{x}_{k, 2}, \mathbf{x}_{k, 3}, \mathbf{x}_{k, 4}$ and tagged by their corresponding digital symbols, 00, 01, 10, and 11. That is, 00 is mapped to $\mathbf{x}_{k, 1}, 01$ to $\mathbf{x}_{k, 2}, 10$ to $\mathbf{x}_{k, 3}$, and 11 is mapped to $\mathbf{x}_{k, 4}$. Each dimension of the 2-dimensional $\mathbf{x}_{k, m}$, i.e., $x_{j, m}$, in Fig. 3.1 is represented by a square with a certain pattern. The binary spreading matrix, $\mathbf{S}_{k}$ is applied on the 2-dimensional complex constellation, $\mathcal{X}_{k}$, to create the 4 dimensional complex codebook, $\mathcal{V}_{k}$. Each complex codeword in Fig. 3.1 corresponds to $\mathbf{v}_{1}$ to $\mathbf{v}_{4}$, tagged by $00,01,10,11$, and represented by four squares. The four squares correspond to the four REs $(N=4)$. That is, each square represents $v_{n, k, m}$, 

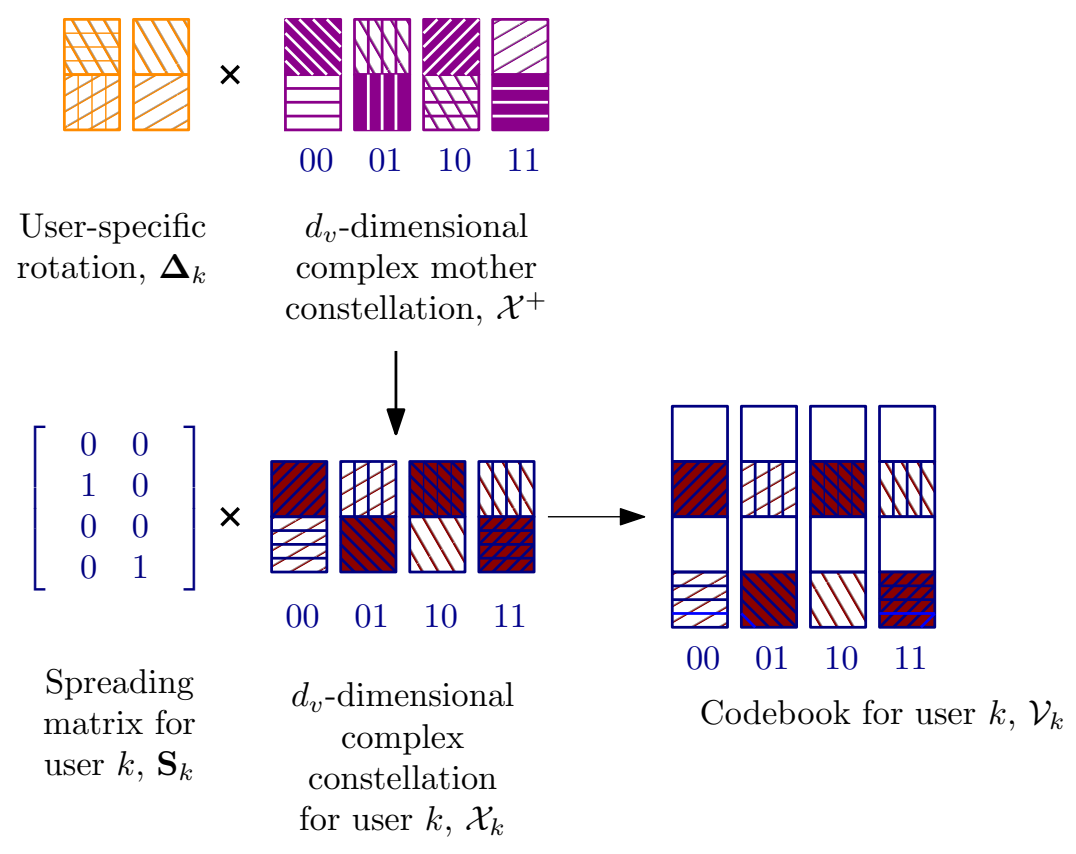

Figure 3.1: Illustration of a sub-optimal process of converting a mother constellation into a user-specific codebook when $d_{v}=4, N=4$, and $M=4$.

$n \in\{1, \ldots, 4\}, k \in\{1, \ldots, 6\}, m \in\{1, \ldots, 4\}$.

The primary purpose of user-specific rotations is to maintain uniquely decodable symbols for users that collide in the same RE. The most commonly employed user-specific rotation on SCMA systems is based on the one proposed in [65] that constitutes a diagonal $\boldsymbol{\Delta}_{k}$. More specifically, the method devised in [65] independently rotates the complex symbol transmitted in each RE assigned to each user, which is independent of the 2-dimensional rotation applied to the other REs assigned to that user. Note that this type of rotation is very important in downlink scenarios, but in uplink scenarios the channel also introduces random rotations to constellations, in which case the optimization of this type of 2-dimensional user-specific rotation does not impact the system performance ${ }^{1}$. That being said, the optimization of the mother constellation $\mathcal{X}^{+}$has a significant impact on the performance of the SCMA system.

In the following section, we highlight the key performance indicators (KPIs) of $M$ point multidimensional constellations for SCMA systems that should be considered in their design process under different channel scenarios. We then provide an overview of different approaches that have been performed to design SCMA multidimensional

\footnotetext{
${ }^{1}$ More details on the effect of user-specific rotations are provided in Appendix B.
} 
constellations in Section 3.4.

\subsection{Key performance indicators}

In this section, we highlight the Key performance indicators (KPIs) that should be considered in the design process of an $M$-point $d_{v}$-dimensional mother constellation, $\mathcal{X}^{+}$, under different channel scenarios provided in Section 2.1. For notational brevity, we drop the superscript + from $\mathcal{X}^{+}$, and denote a $d_{v^{-}}$dimensional complex mother constellation by $\mathcal{X}$. Each constellation point is then represented by $\mathbf{x}_{m}$.

Multidimensional constellations can be projected onto a set of orthogonal 2dimensional signal spaces (i.e., the I and Q channels) where each projection can be transmitted independently (e.g., [66]). In the context of SCMA, each of the orthogonal 2-dimensional signal spaces refers to one RE. We represent the projection of the $j$ th complex element of $\mathbf{x}_{m}$ onto two dimensions by $x_{m j}, 1 \leq j \leq d_{v}$. For a scenario with $d_{v}=2$, consider the 4-point 2-dimensional complex constellation in Table 3.1. As we see, 00 is mapped to $(+1,0), 01$ to $(0,+i), 10$ to $(0,-i)$, and 11 to $(-1,0)$. The projection of each constellation point onto the first pre-assigned $\mathrm{RE}\left(\mathrm{RE}_{1}\right)$ and onto the second pre-assigned $\mathrm{RE}\left(\mathrm{RE}_{2}\right)$ are denoted by $x_{m 1}$ and $x_{m 2}$ in Table 3.1, and depicted in Fig. 3.2.

The average symbol energy of a constellation is defined as

$$
E_{\mathrm{S}}=\frac{1}{M} \sum_{m=1}^{M}\left\|\mathbf{x}_{m}\right\|^{2},
$$

where $\|\cdot\|$ denote the $L^{2}$-norm. In this thesis, we have normalized the energies of all constellations to unity in order to provide a consistent comparison.

The KPIs of $M$-point $d_{v}$-dimensional complex constellations that significantly impact the performance of SCMA systems over different channel scenarios are as follows:

\section{Euclidean Distance}

The Euclidean distance between two constellation points, $\mathbf{x}_{m}$ and $\mathbf{x}_{m^{\prime}}, 1 \leq m<m^{\prime} \leq$ $M$, is

$$
d_{E}^{m m^{\prime}}=\left\|\mathbf{x}_{m}-\mathbf{x}_{m^{\prime}}\right\|,
$$


Table 3.1: An example of a 4-point 2-dimensional complex constellation

\begin{tabular}{ccc}
\hline$m-1$ & $x_{m 1}$ & $x_{m 2}$ \\
\hline 00 & +1 & 0 \\
01 & 0 & $+i$ \\
10 & 0 & $-i$ \\
11 & -1 & 0 \\
\hline
\end{tabular}
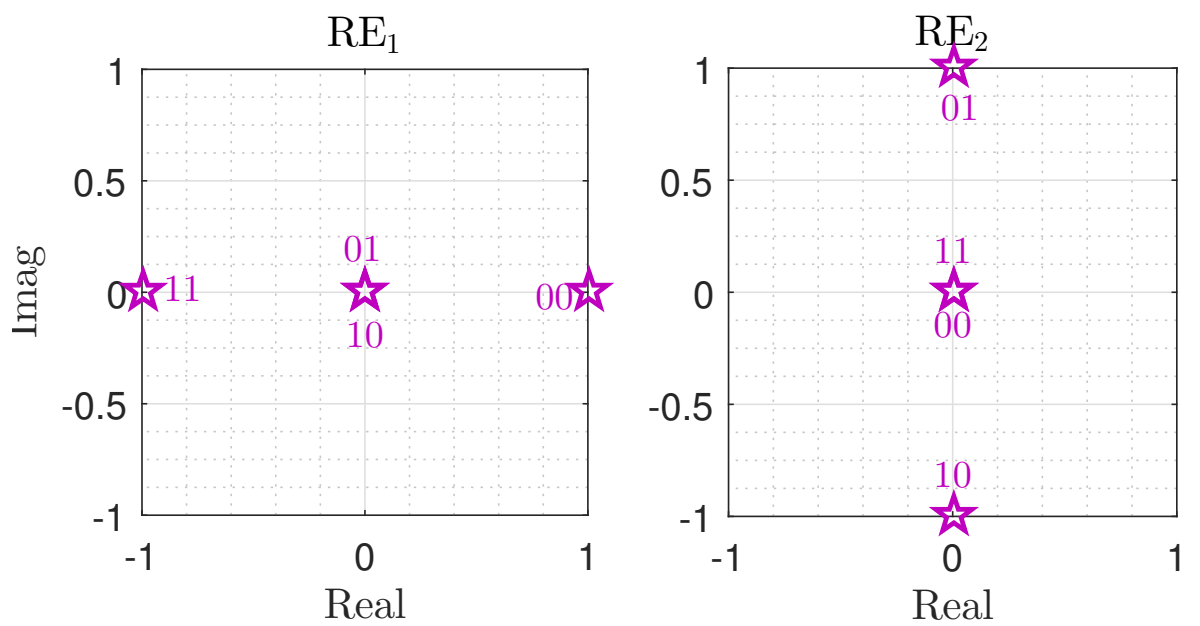

Figure 3.2: The projection of a 4-point 2-dimensional complex constellation onto the first pre-assigned $\mathrm{RE}\left(\mathrm{RE}_{1}\right)$, and the second pre-assigned $\mathrm{RE}\left(\mathrm{RE}_{2}\right)$.

The minimum Euclidean distance of a constellation is defined as

$$
d_{E, \min }=\min \left\{d_{E}^{m m^{\prime}} \mid 1 \leq m<m^{\prime} \leq M\right\}
$$

It is known that the minimum Euclidean distance of a constellation is a KPI in AWGN channels [67-69]. Moreover, in fading scenarios with $d_{v}$ adjacent REs (dependent fading) since the constellation points over the $d_{v}$ REs are rotated and scaled by the same values (the same channel coefficients), the relative Euclidean distance between the constellation points remains intact. As such, $d_{E \text {, min }}$ is an important factor in FSC, FFSC and SFSC. 


\section{Euclidean Kissing Number}

The Euclidean kissing number, $\tau_{E}$, is defined as the average number of constellation pairs at the minimum Euclidean distance $d_{E \text {, min }}$, and is a KPI in FSC, FFSC, and SFSC.

\section{Product Distance}

The product distance between two $d_{v}$-dimensional complex constellation points, $\mathbf{x}_{m}$ and $\mathbf{x}_{m^{\prime}}$, is defined as [67]

$$
d_{P}^{m m^{\prime}}=\prod_{j \in \mathcal{J}_{m m^{\prime}}}\left|x_{m j}-x_{m^{\prime} j}\right|
$$

where $x_{m j}$ and $x_{m^{\prime} j}$ are the $j$ th complex element of $\mathbf{x}_{m}$ and $\mathbf{x}_{m^{\prime}}$, respectively. Moreover, $\mathcal{J}_{m m^{\prime}}$ denotes the set of dimensions, $j$, for which $x_{m j} \neq x_{m^{\prime} j}$, i.e.,

$$
\mathcal{J}_{m m^{\prime}}=\left\{j \mid x_{m j} \neq x_{m^{\prime}}, 1 \leq j \leq d_{v}\right\}
$$

The minimum product distance is

$$
d_{P, \min }=\min \left\{d_{P}^{m m^{\prime}} \mid 1 \leq m<m^{\prime} \leq M\right\}
$$

It is known from [69-71] that maximizing the minimum product distance of the constellation points has an important impact on the performance of the system in fading scenarios with $d_{v}$ non-adjacent REs (independent fading), i.e., FIC, FFIC, SFIC, especially at high SNRs.

\section{Product Kissing Number}

The product kissing number, $\tau_{P}$, is defined as the average number of constellation

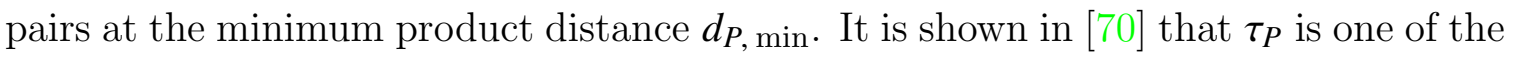
dominant factors in the symbol error probability of a multidimensional constellation in fading scenarios with $d_{v}$ non-adjacent REs, i.e., FIC, FFIC, SFIC.

\section{Modulation Diversity Order}

The modulation/signal space diversity order, $L$, of a multidimensional constellation is defined as the minimum number of distinct dimensions between any two constellation 
points [71]. In other words, $L$ is the minimum Hamming distance between any two different constellation points. That is,

$$
L=\min \left\{d_{H}\left(\mathbf{x}_{m}, \mathbf{x}_{m^{\prime}}\right) \mid 1 \leq m<m^{\prime} \leq M\right\}
$$

where $d_{H}\left(\mathbf{x}_{m}, \mathbf{x}_{m^{\prime}}\right)$ represents the Hamming distance between $\mathbf{x}_{m}$ and $\mathbf{x}_{m^{\prime}}$, i.e., the cardinality of $\mathcal{J}_{m m^{\prime}}$ in $(3.10)$. It is known (e.g., $\left.[69,71,72]\right)$ that $L$ plays an important role in fading channels with $d_{v}$ non-adjacent REs, i.e., FIC, FFIC, SFIC. ${ }^{2}$

\section{Number of Distinct Points}

In SCMA systems, the average number of distinct points, $N_{d}, 0<N_{d} \leq M$, along the projections of an $M$-point $d_{v}$-dimensional complex constellation onto two dimensions (I and Q channels) is a KPI in most scenarios, and is defined as

$$
N_{d}=\frac{1}{d_{v}} \sum_{j=1}^{d_{v}} \sum_{m=1}^{M} \delta\left[\sum_{m^{\prime}<m} C\left(\left|x_{m j}-x_{m^{\prime} j}\right|\right)\right],
$$

where

$$
C(z)=\left\{\begin{array}{cc}
1 & \text { if } z=0 \\
0 & \text { if } z \neq 0
\end{array},\right.
$$

and $\delta[\cdot]$ denotes the Kronecker delta function.

For the constellation provided in Table 3.1 and depicted in Fig. 3.2, it is clear that both 01 and 10 overlap over $\mathrm{RE}_{1}$, and both 00 and 11 overlap over $\mathrm{RE}_{2}$. Referring to (3.13), for this constellation $N_{d}=3$.

As we will discuss later in Section 3.5, in general, the higher $N_{d}$, the better the performance. That is, if there is an overlap among different symbols, i.e., $N_{d} \neq M$, in a scenario where one of the REs is in a deep fade, the transmitted symbol cannot easily be recovered from other REs.

\footnotetext{
${ }^{2}$ It is worthwhile to note that the fading channel diversity order is equal to $L$ in fading channels with $d_{v}$ non-adjacent REs, i.e., FIC, FFIC, SFIC, whereas the fading channel diversity order is equal to 1 in fading channels with $d_{v}$ adjacent REs, i.e., FSC, FFSC, SFSC.
} 


\section{Bit-labeling}

In uncoded systems, the mapping function that maps the incoming bits to each constellation point, i.e., bit-labeling, has an impact on the BER performance. However, bit-labeling has no impact on the symbol error performance (SER) of uncoded systems [67]. In coded systems, bit-labeling plays an important role on the system performance in different channel scenarios (e.g., [73]). This can be due to the fact that in many coded systems, the output of the detector is used to calculate the bit LLRs, which are then fed to the channel decoder. As such, the BER performance of the uncoded system gives some indication of the system performance in the presence of the channel coding.

Bit-labeling for BICM has been studied widely e.g., [72, 74-79]. In [79], it is conjectured that Gray labeling maximizes the capacity of BICM. In Gray labeling, two points in the constellations that are adjacent (in terms of the Euclidean distance) must differ in only one bit position. The multidimensional constellations based on hypercubes, e.g., constellations constructed similar to [71], with Gray labeling provide a nearly equal error protection among their different bit levels. As such, they perform well when employed in conjunction with BICM. Note that by Gray labeling of the $d_{v}$-dimensional constellation, we mean Gray labeling in the $d_{v}$-dimensional space, and not necessarily Gray labeling the individual projections of the constellation onto each RE.

In addition to the KPIs mentioned above, the behavior of the multiuser detector at different SNR regions can accordingly effect the behavior of constellations in different scenarios. Moreover, the code rate has a significant impact on the system performance.

\subsection{Overview of SCMA Multidimensional Constel- lations}

In this section, we provide an overview of the $M$-point $2 d_{v}$-dimensional real constellations for uplink SCMA systems proposed in [21,30,51-64] for $M \in\{4,16\}$, and $d_{v}=2$. That is, each user sends its data over two REs only ${ }^{3}$. The $M$-point $2 d_{v}$-dimensional

\footnotetext{
${ }^{3}$ Note that most of the constellations proposed for SCMA systems are given for $M \in\{4,16\}, d_{v}=$ 2. As an essential purpose of SCMA systems is to support massive connectivity in IoT applications [80], and not increasing the throughput of individual users, $M \in\{4,16\}$ is a fair assumption. In
} 
constellations under study are as follows:

\section{$M-\mathrm{LDS}$}

The repetition of an $M$-QAM constellation over the 2 REs, which is referred as $M$ LDS [21]. In Fig. 3.3(a), we show the projection of 4-LDS with magenta left-pointing triangles over $\mathrm{RE}_{1}$ and $\mathrm{RE}_{2}$. As an example, with a Gray labeling, to transmit 11 a user would send $(-1 / 2,-1 / 2)$ over both $\mathrm{RE}_{1}$ and $\mathrm{RE}_{2}$. Likewise, we depict the projection of 16-LDS with magenta left-pointing triangles over $\mathrm{RE}_{1}$ and $\mathrm{RE}_{2}$ in Fig. 3.6(a) and Fig. 3.6(b), respectively.

\section{TMQAM}

The constellation proposed in [30,51]. Inspired by [71], the design process of this constellation involves a shuffling method that establishes a $d_{v}$-dimensional complex constellations from the Cartesian product of two $d_{v}$-dimensional real constellations with a desired Euclidean distance profile. A unitary rotation matrix is then applied to maximize the minimum product distance, $d_{P}$, min , of the $2 d_{v}$-dimensional constellation. This $M$-point constellation was named as TMQAM when it was proposed in $[30,51]$.

We show the projections of T4QAM onto two dimensions with red circles in Fig. 3.3(b). Each user maps the incoming bitstreams onto the constellation with red circles in Fig. 3.3(b) in order to send them over $\mathrm{RE}_{1}$ and $\mathrm{RE}_{2}$. For example, to transmit 11 , the user would send $(-3 / \sqrt{10}, 0)$ on its first RE and $(-1 / \sqrt{10}, 0)$ on its second RE. Furthermore, we show the projections of T16QAM onto two dimensions for the two REs with red circles in Fig. 3.7(a)-Fig. 3.7(b). As an example, with a Gray labeling to transmit 1111 , the user sends $(-3 \sqrt{5} / 10,-3 \sqrt{5} / 10)$ and $(-\sqrt{5} / 10,-\sqrt{5} / 10)$ on its $\mathrm{RE}_{1}$ and its $\mathrm{RE}_{2}$, respectively.

It is worth to note that the mother constellation of the widely used (e.g., [8184]) SCMA codebook that is represented in [1], and later described in [52], is in fact the T4QAM constellation. However, in [1,52] the 2-dimensional components of the mother constellation are then rotated according to the Latin square criterion

addition, as the user-to-RE indicator matrix for an SCMA system is required to be a sparse matrix, $d_{v}=2$ is an important scenario. 

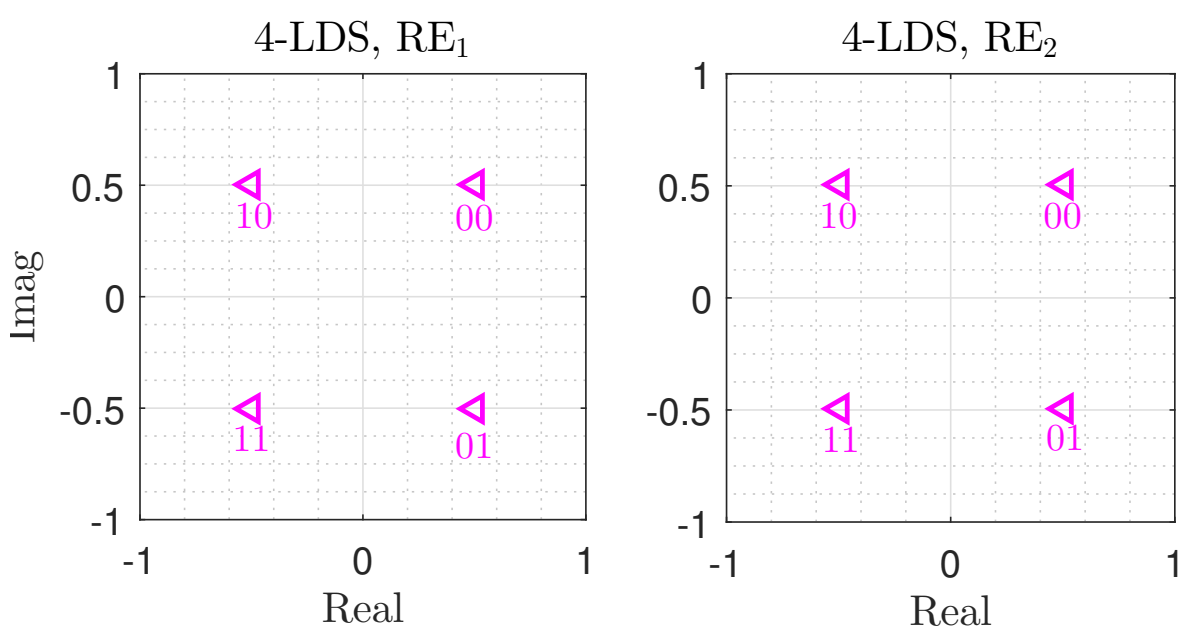

(a)
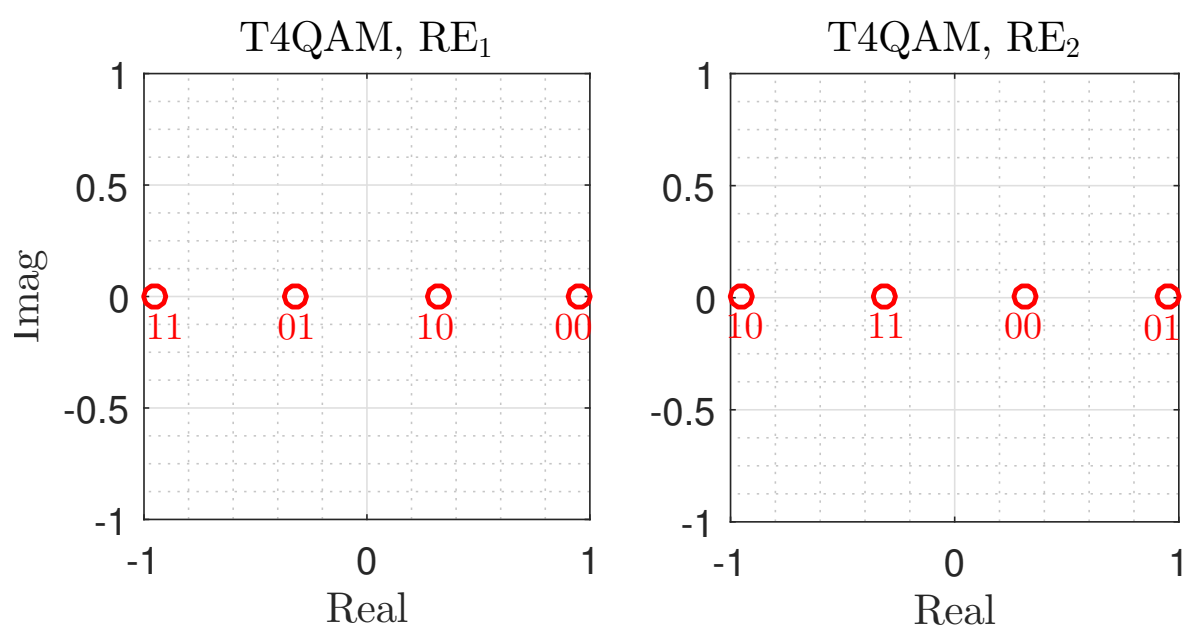

(b)

Figure 3.3: The projection of 4-point 4-dimensional constellations over $\mathrm{RE}_{1}$ and $\mathrm{RE}_{2}$ : (a) 4-LDS, and (b) T4QAM. 

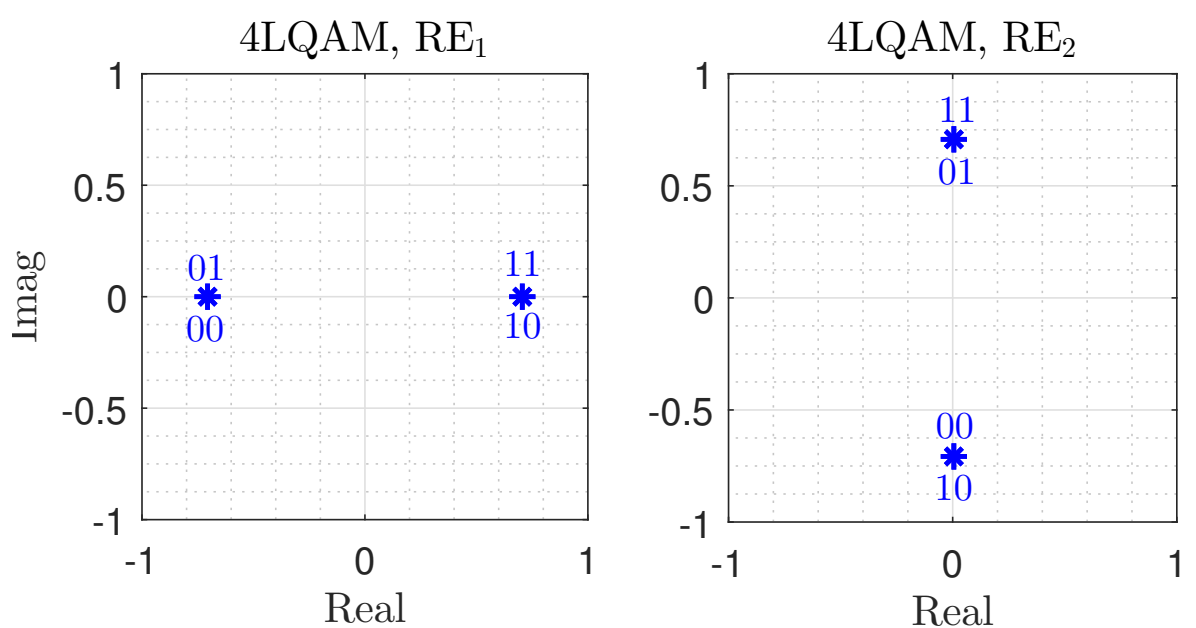

(a)
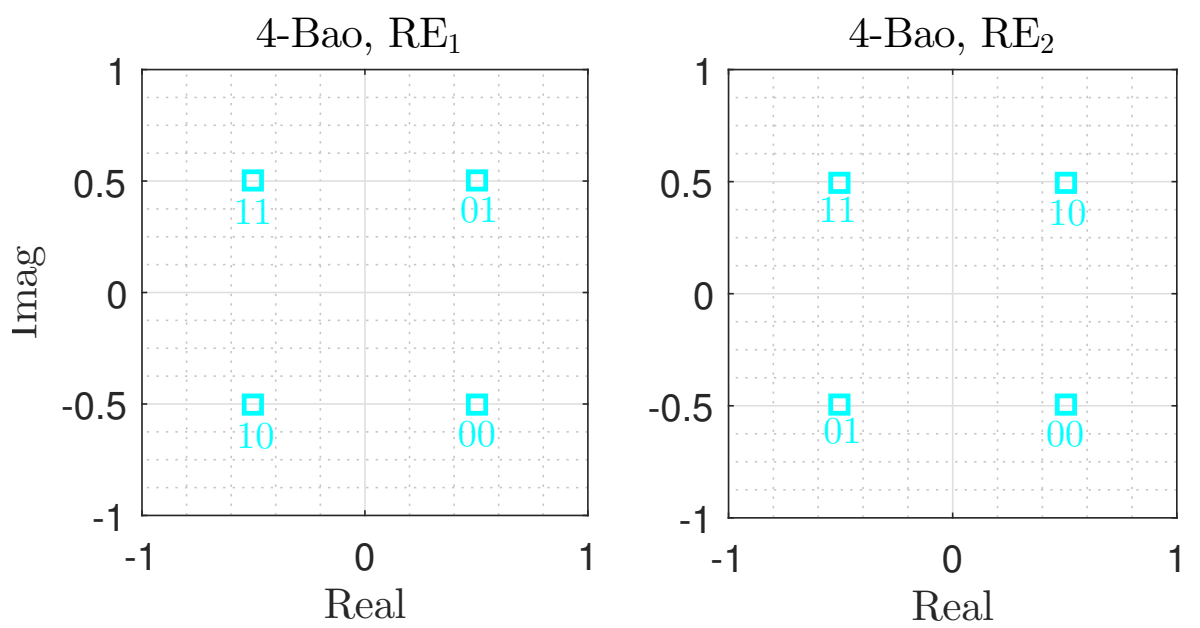

(b)

Figure 3.4: The projection of 4-point 4-dimensional constellations over $\mathrm{RE}_{1}$ and $\mathrm{RE}_{2}$ : (a) 4LQAM, and (b) 4-Bao. 

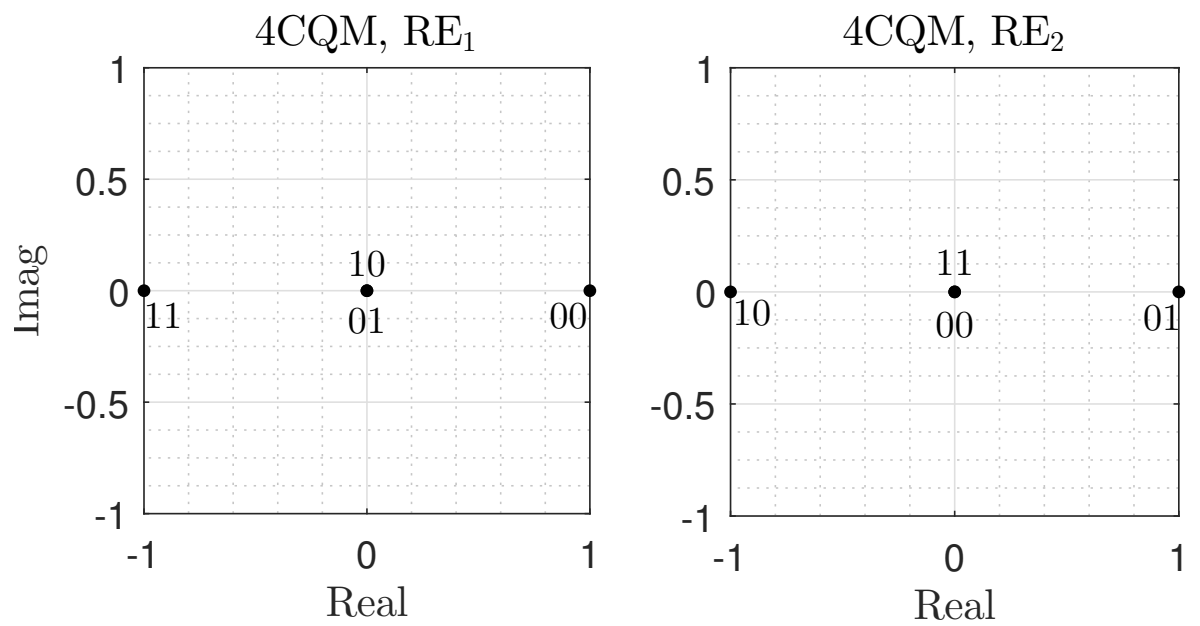

(a)
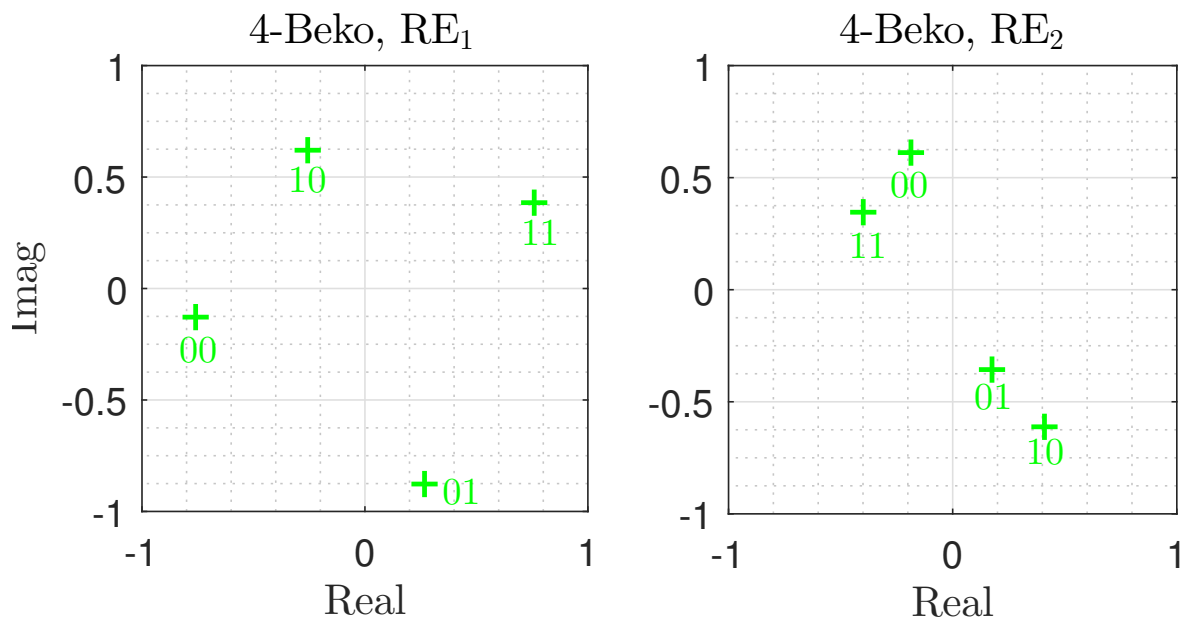

(b)

Figure 3.5: The projection of 4-point 4-dimensional constellations over $\mathrm{RE}_{1}$ and $\mathrm{RE}_{2}$ : (a) 4CQAM, and (b) 4-Beko. 


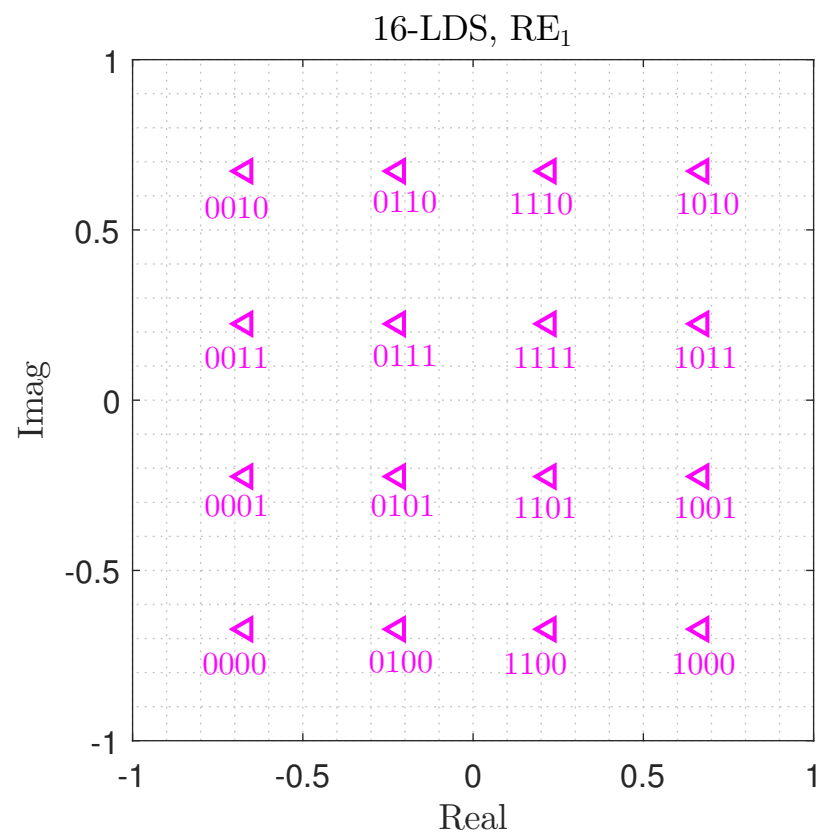

(a)

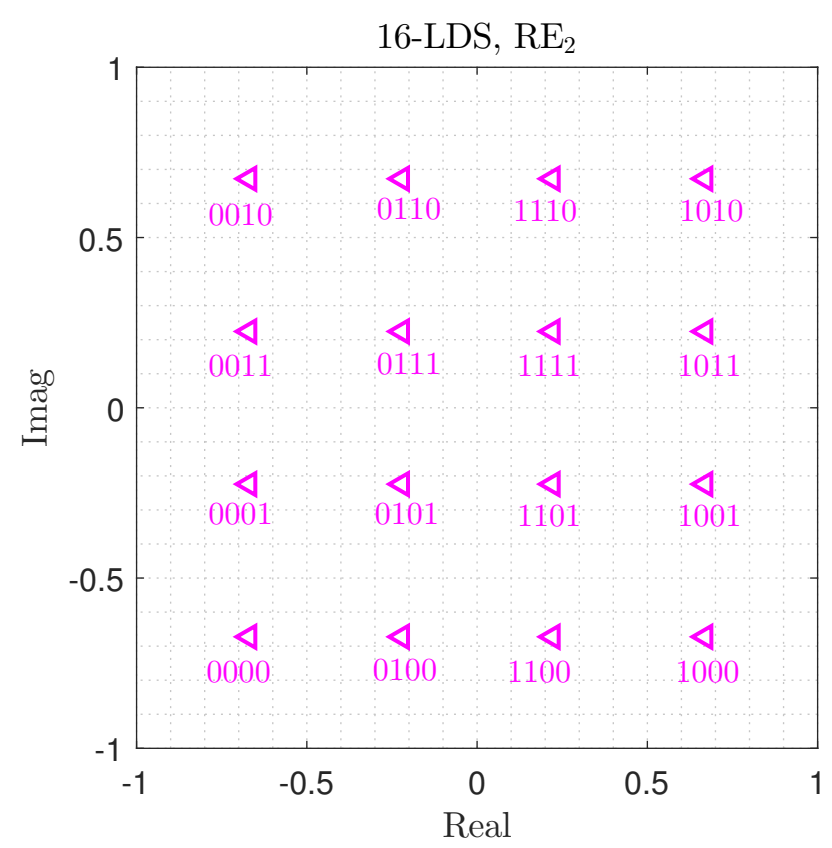

(b)

Figure 3.6: The projection of the 16-LDS constellation over (a) $R_{1}$, and (b) $R_{2}$. 
[85] to obtain multidimensional constellations that are different for different users. Furthermore, the 4-point mother constellation that is proposed in [53] is similar to T4QAM, and is rotated according to [65] to differentiate the users. As mentioned earlier, the value of the 2-dimensional user-specific rotations becomes questionable in uplink scenarios. In addition, the codebook that is proposed in [54] is based on the technique devised in [71], and is expected to result in a constellation similar to TMQAM.

\section{$M$ LQAM}

The constellation with a low number of projection that is proposed in $[30,51]$, and given in [55]. We refer to the $\boldsymbol{M}$-point constellation in [30,51] as $M$ LQAM (QAM with a low number of projections). Similar to TMQAM, the design process of MLQAM associates with a shuffling method that constitutes a $d_{v}$-dimensional complex constellations from the Cartesian product of two $d_{v}$-dimensional real constellations. That said, unlike TMQAM, a unitary rotation matrix is then applied to lower the number of projected points over each RE, which, in turn, decreases the computational complexity of the detector [41].

We show the projections of 4LQAM onto two dimensions over $\mathrm{RE}_{1}$ and $\mathrm{RE}_{2}$ with blue asterisks in Fig. 3.4(a). As we see in Fig. 3.4(a), 4 points of the constellation are mapped to $N_{d}=2$ points only. That is, with Gray labeling, both 00 and 01 are mapped to $(-\sqrt{2} / 2,0)$, and both 10 and 11 are mapped to $(\sqrt{2} / 2,0)$ over $R_{1}$. Moreover, both 00 and 10 are mapped to $(0,-\sqrt{2} / 2)$, and both 01 and 11 are mapped to $(0, \sqrt{2} / 2)$ over $\mathrm{RE}_{2}$. As such, the MPA detection computational complexity [30] can be reduced from $4^{d_{c}}$ to $2^{d_{c}}$. The $4 \mathrm{LQAM}$ constellation in Fig. $4(\mathrm{c})$ can be considered as the transmission of two independent BPSK constellations on each RE. In a similar vein, we depict the projections of 16LQAM onto two dimensions over $\mathrm{RE}_{1}$ and $\mathrm{RE}_{2}$ with blue asterisks in Fig. 3.8(a) and Fig. 3.8(b), respectively. It is clear that 16 points of the constellation are mapped to only $N_{d}=9$ points in each 2-dimensional component. Therefore, the MPA detection computational complexity can be reduced from $16^{d_{c}}$ to $9^{d_{c}}$. Nevertheless, as we will discuss in Section 3.5, 4LQAM and 16LQAM introduce performance degradations in some scenarios. 


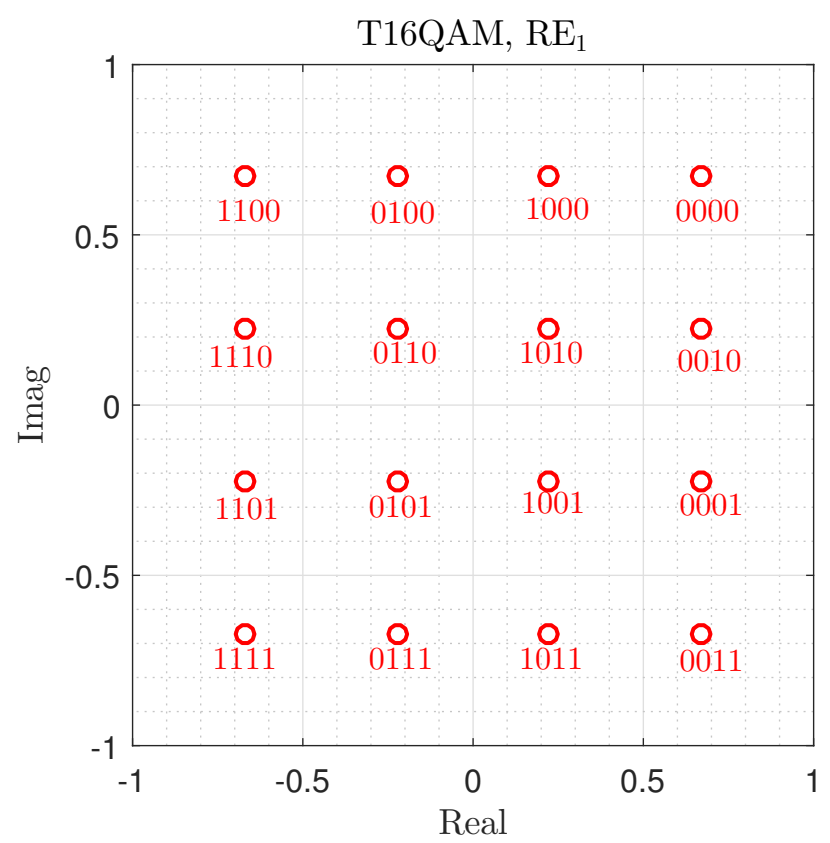

(a)

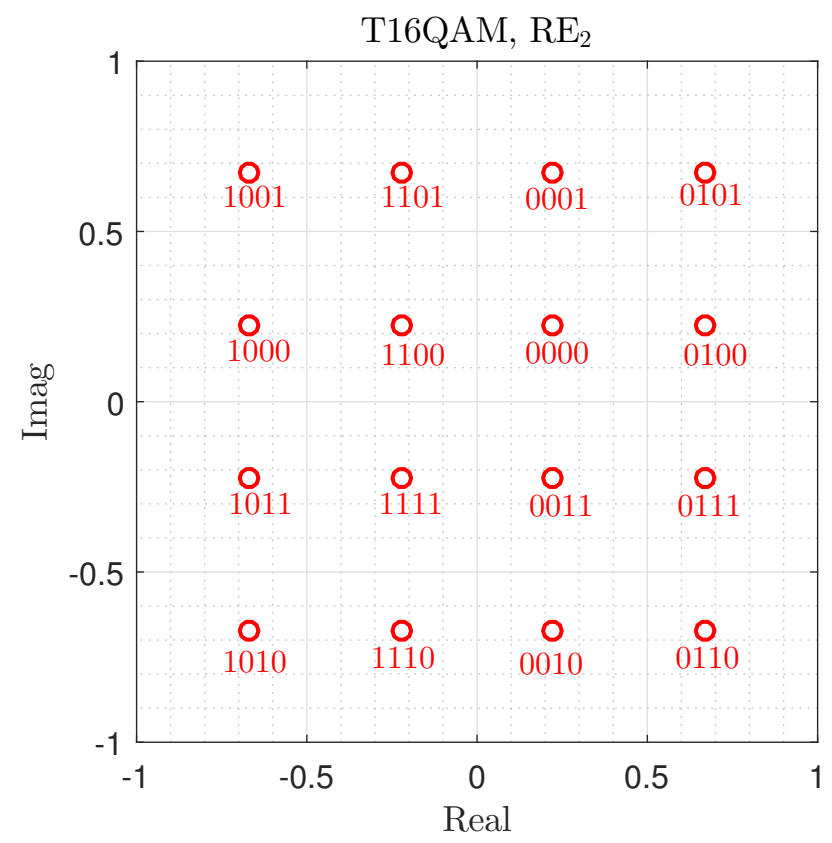

(b)

Figure 3.7: The projection of the T16QAM constellation over (a) $\mathrm{RE}_{1}$, and (b) $\mathrm{RE}_{2}$. 


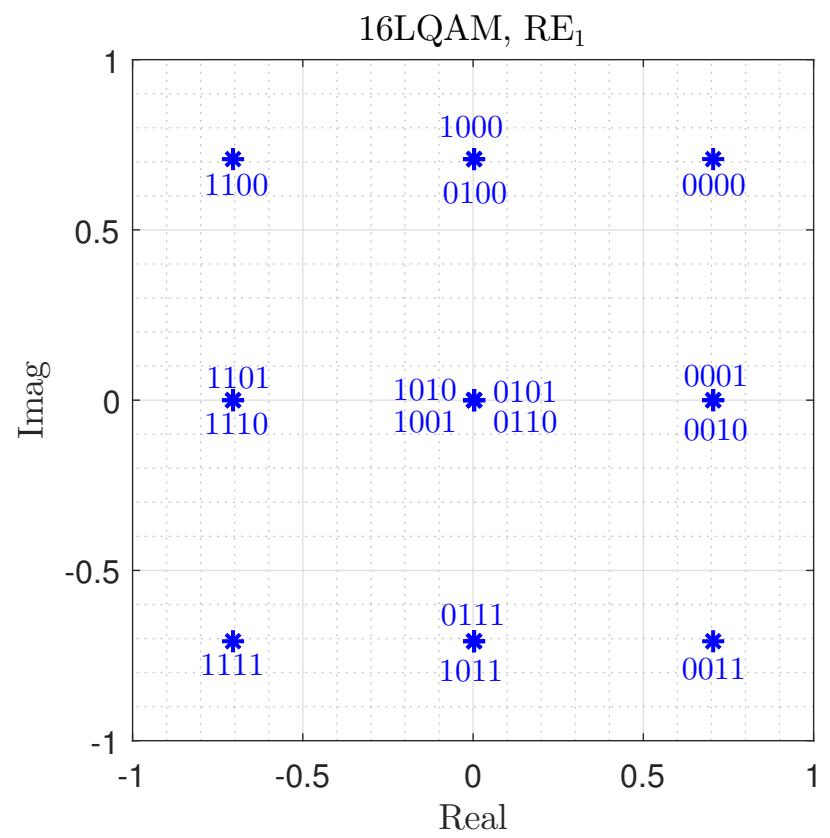

(a)

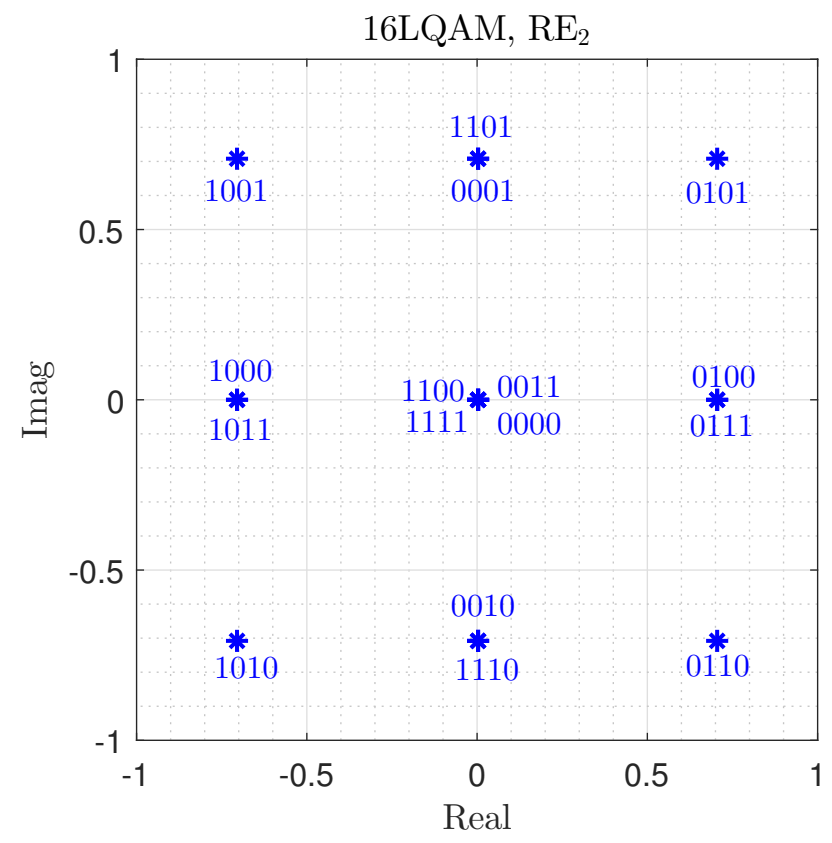

(b)

Figure 3.8: The projection of the 16LQAM constellation over (a) $R_{1}$, and (b) $R_{2}$. 


\section{$M-\mathrm{Bao}$}

The constellation proposed for SCMA systems over Rayleigh fading channels with $d_{v}$ non-adjacent REs, i.e., FIC (Case 2), in [56] that is based on the rotation of QAM constellations. The rotation matrices are obtained through computer search inspired by the approaches in $[86,87]$ in order to maximize the cutoff rate of the equivalent MIMO systems. This exhaustive search is only feasible if $d_{c} \leq 3$. We name the $M$-point constellation proposed in [56] as $M$-Bao.

We depict the projection of four-dimensional 4-Bao onto two dimensions over $\mathrm{RE}_{1}$ and $\mathrm{RE}_{2}$ with cyan squares in Fig. 3.4(b). With a Gray labeling, to send 01 , the user would send $(0.5019,0.4981)$ over $\mathrm{RE}_{1}$, and $(-0.5019,-0.4981)$ over $\mathrm{RE}_{2}$. Further, we show the projection of four-dimensional 16-Bao onto two dimensions over $\mathrm{RE}_{1}$ and $\mathrm{RE}_{2}$ with cyan squares in Fig. 3.9(a) and Fig. 3.9(b), respectively. For instance, with a Gray labeling, to send 0001, the user would send $(0.3876,-0.8148)$ and $(-0.1890,-0.3876)$ over $\mathrm{RE}_{1}$ and $\mathrm{RE}_{2}$, respectively.

In [57], the spherical codes are proposed for SCMA systems that are based on the constellations in $[88,89]$. It was later shown in [56] by similar authors that $M$-Bao in [56] outperforms the spherical codes in [57].

\section{HQAM}

By investigating T16QAM, 16LQAM, and 16-Bao, we notice they all are based on the Cartesian product of two 4-QAM constellations that are rotated using different unitary rotations to fulfill different aforementioned requirements. The Cartesian product of two 4-QAM constellations constitutes the 16 corners of the four-dimensional hypercube. We name the 16-point four-dimensional hypercube-based QAM as 16HQAM, and depict its projection onto two dimensions over $\mathrm{RE}_{1}$ and $\mathrm{RE}_{2}$ with brown crosses in Fig. 3.10(a) and Fig. 3.10(b), respectively. As we see, the 16 points of the constellations are mapped to $N_{d}=4$ points only, which can result in a reduction of MPA

detection complexity from $16^{d_{c}}$ to $4^{d_{c}}$. That said, as we discuss in Section 3.5, the performance of 16HQAM will be penalized in some scenarios by its low number of distinct points. 


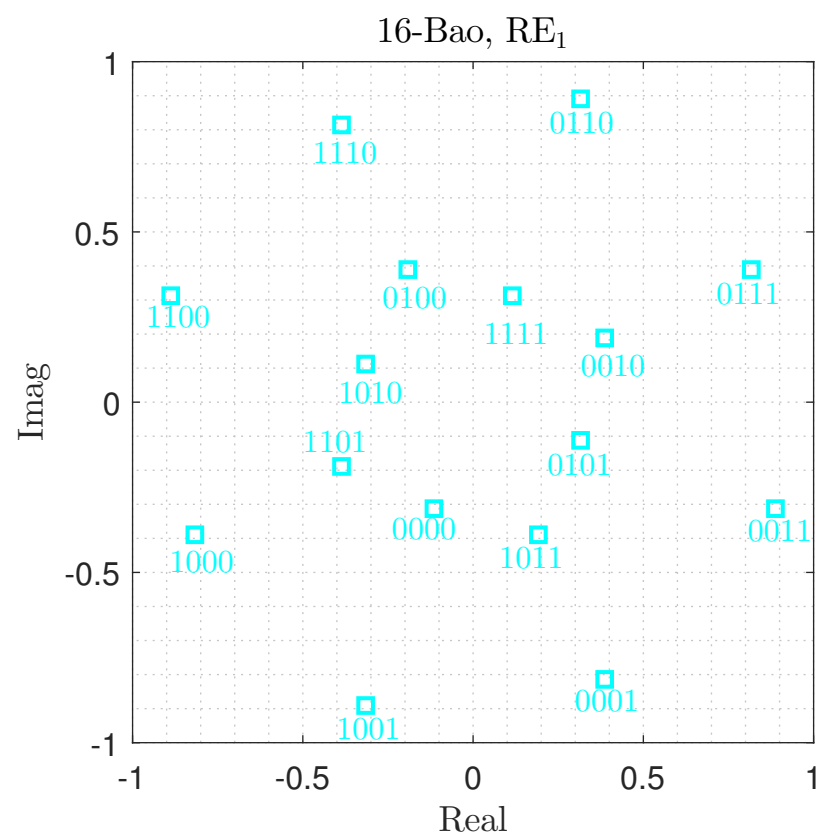

(a)

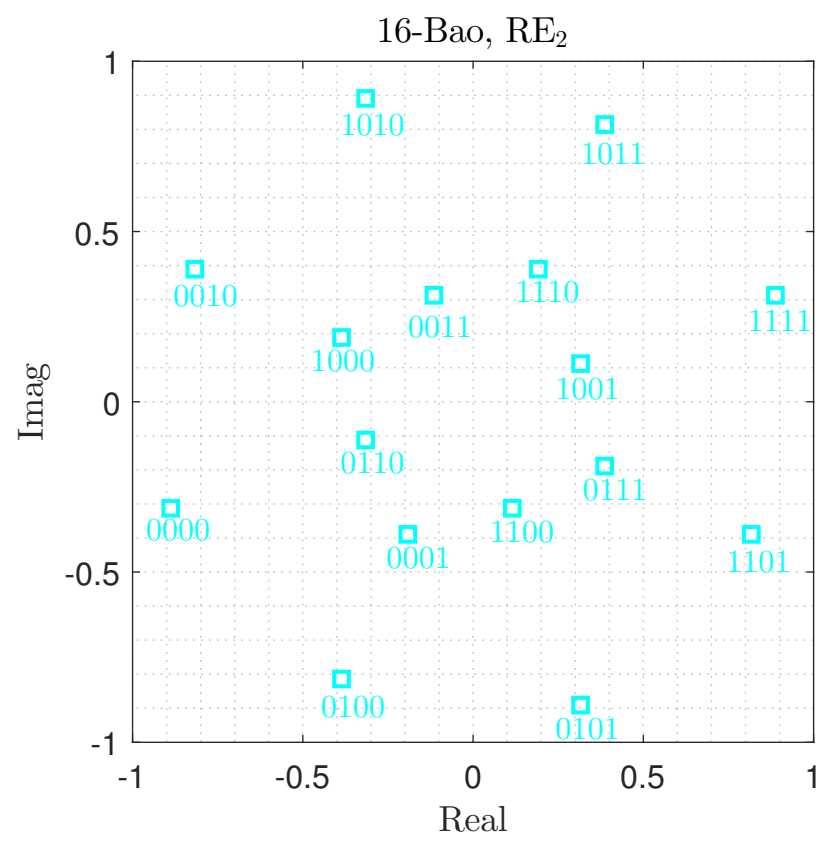

(b)

Figure 3.9: The projection of the 16-Bao constellation over (a) $\mathrm{RE}_{1}$, and (b) $\mathrm{RE}_{2}$. 


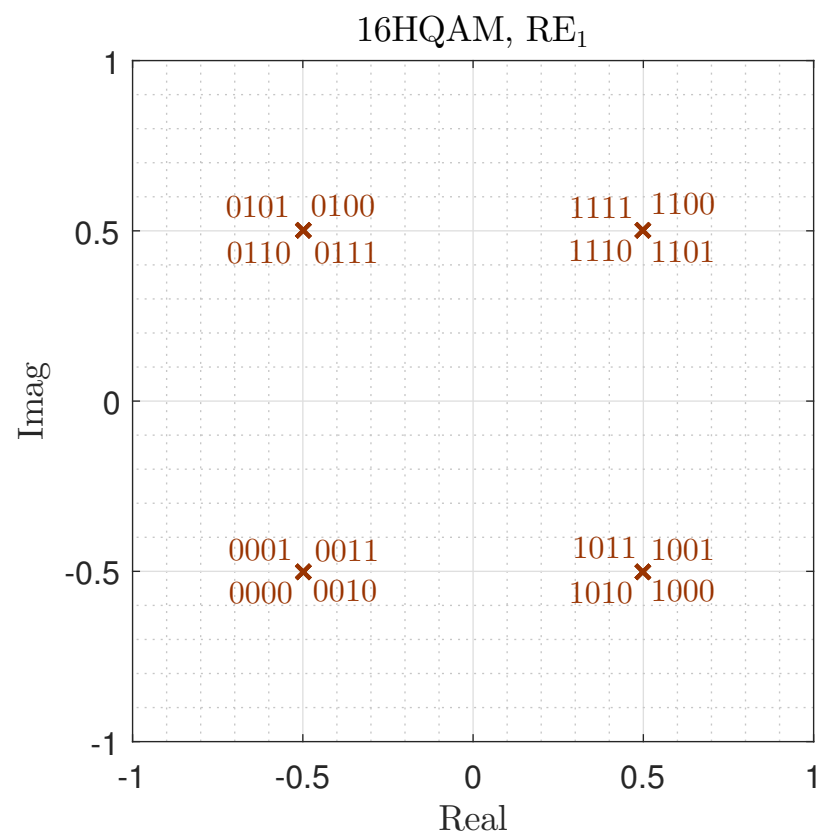

(a)

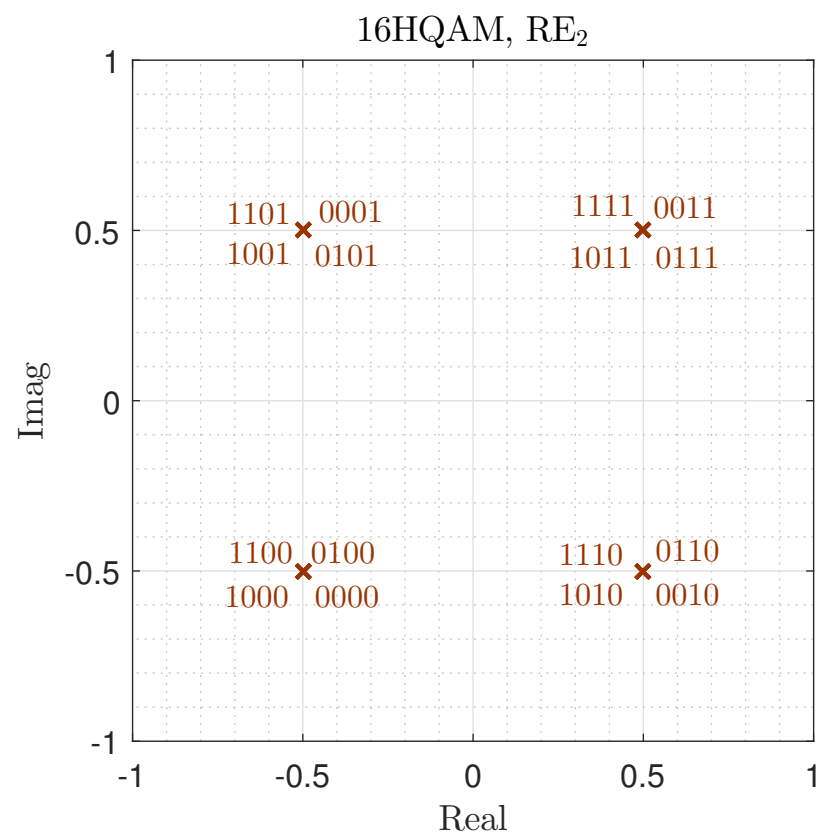

(b)

Figure 3.10: The projection of the 16HQAM constellation over (a) $\mathrm{RE}_{1}$, and (b) $\mathrm{RE}_{2}$ 


\section{$M$ CQAM}

The circular QAM constellation proposed in [55]. We refer to $M$-point circular QAM constellation as MCQAM. MCQAM is based on the analysis of the signal-space diversity for MIMO systems over Rayleigh fading channels in $[42,56]$, but it also obtains a low number of projections per complex dimension.

We show the projection of $4 \mathrm{CQAM}^{4}$ over $\mathrm{RE}_{1}$ and $\mathrm{RE}_{2}$ with black points in Fig. 3.5(a). As we see, the 4 points of the constellation are mapped to $N_{d}=3$ points. More specifically, with a Gray labeling, in order to transmit over $\mathrm{RE}_{1}$, both 01 and 10 are mapped to $(0,0), 00$ is mapped to $(1,0)$, and 11 is mapped to $(-1,0)$. On the other hand, to transmit over $\mathrm{RE}_{2}$, both 00 and 11 are mapped to $(0,0), 01$ is mapped to $(1,0)$, and 10 is mapped to $(-1,0)$. Hence, the MPA detection computational complexity can be reduced from $4^{d_{c}}$ to $3^{d_{c}}$. Similarly, we depict the projection of 16CQAM over $\mathrm{RE}_{1}$ and $\mathrm{RE}_{2}$ with black points in Fig. 3.11(a) and Fig. 3.11(b), respectively. We observe that the 16 points of the constellation are mapped to $N_{d}=9$ points only. The MPA detection computation is proportional to $9^{d_{c}}$ instead of $16^{d_{c}}$. However, akin to MLQAM and 16HQAM, MCQM suffers from a performance degradation in some scenarios (as will be discussed in Section 3.5).

In [58], an adaptive codebook design and assignment is proposed for SCMA systems that aims to reduce the energy consumption caused by the detection process. The proposed mother constellation has a similar structure as the CQAM constellation. The two-dimensional user-specific rotations are then applied to differentiate the users. As mentioned earlier in Section 3.2, those user-specific rotations become questionable in uplink scenarios.

$A_{M, N_{d}}$

The $M$-point constellation proposed in [59] with a desired $N_{d}$ distinct points, and is referred as $A_{M, N_{d}}$. Based on the extrinsic information transfer (EXIT) chart, it is shown in [59] that the impact of the constellation and labeling on a single-user system is consistent with that on a multiuser system. In other words, a good constellation and labeling for a single-user system is also good for the multiuser case. A multistage optimization for designing a $2 d_{v}$-dimensional real constellation with a low number of projections is then proposed that consists of the following main steps: 1) For a desired

\footnotetext{
${ }^{4}$ This constellation is also provided in [90].
} 


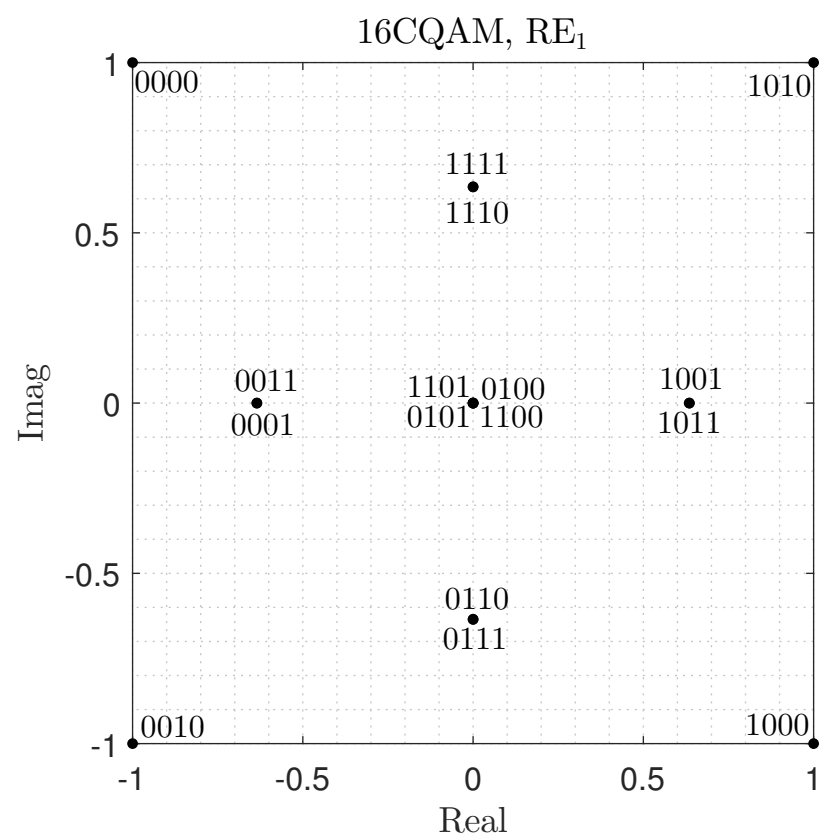

(a)

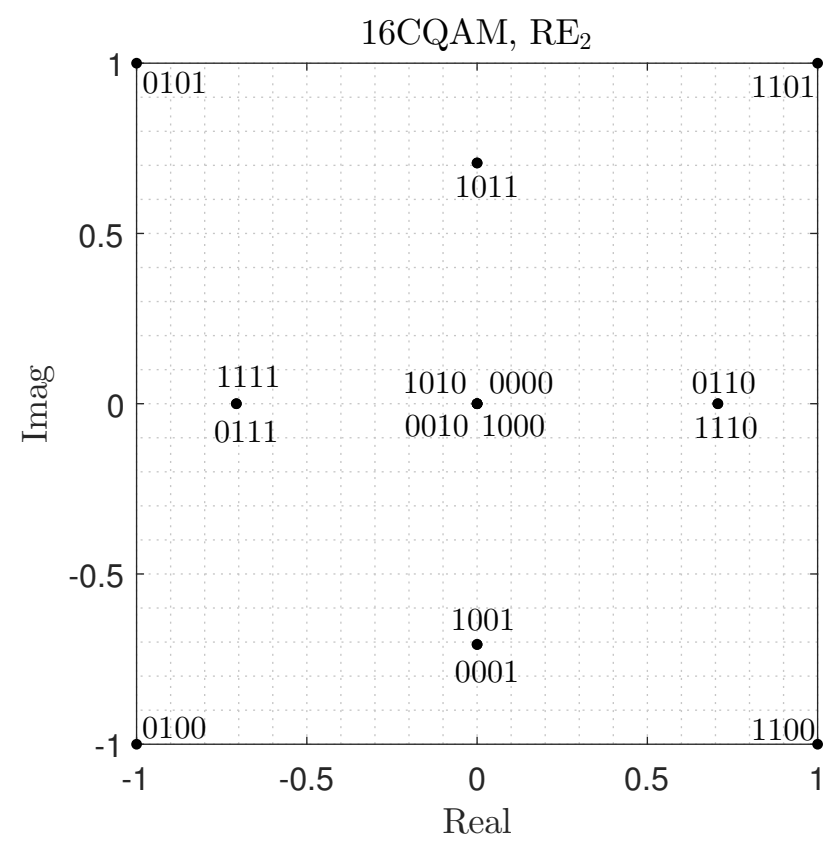

(b)

Figure 3.11: The projection of the 16CQAM constellation over (a) $\mathrm{RE}_{1}$, and (b) $\mathrm{RE}_{2}$. 
$N_{d}$, a one-dimensional constellation, $\mathcal{A}$, with a good average mutual information (AMI), is used as a base constellation. The $N_{d}$ amplitude phase-shift keying $\left(N_{d^{-}}\right.$ APSK) constellation can be constructed using the general APSK procedure in [91], and provides a good AMI. Hence, it is used as the base constellation. 2) Based on the one-dimensional $\mathcal{A}$, a $2 d_{v}$-dimensional real constellation is constructed using a permutation function that is provided in [59]. 3) An appropriate labeling based on the EXIT chart is then optimized for the resultant $2 d_{v}$-dimensional real constellation.

As an example, we show the projection of the 16-point 4-dimensional constellation proposed in [59] with $N_{d}=5$, i.e., $A_{16,5}$, over $\mathrm{RE}_{1}$ and $\mathrm{RE}_{2}$ in Fig. 3.12(a) and Fig. 3.12(b), respectively.

In [60], an $M$-point constellation with low number of projections is proposed for non-binary low density parity check (LDPC) coded SCMA that aims to maximize the minimum Euclidean distance, $d_{E \text {, min }}$, between the constellation points. As mentioned in Section 3.3, maximizing $d_{E \text {, min }}$ is not a KPI in fading scenarios with independent fading over the $d_{v}$ REs.

\section{$M$-Beko}

The $M$-point constellation proposed in [68] for uncoded systems over AWGN channels, by minimizing the average symbol energy for a given $d_{E \text {, min }}$ between constellation points. The design of this constellation is formulated as a non-convex optimization problem that is tackled by solving a sequence of convex optimization problems. The indicated constellation is later used in the context of SCMA systems in [61]. We name the $M$-point constellation proposed in [68] as $M$-Beko ${ }^{5}$.

We depict the projection of 4-Beko onto two dimensions with green plus signs over $\mathrm{RE}_{1}$ and $\mathrm{RE}_{2}$ in Fig. 3.5(b). As an example, to transmit 11 the user sends $(0.7543,0.3852)$ and $(-0.3993,0.3509)$ over $R_{1}$ and $R E_{2}$, respectively. In a similar vein, we show projection of 16-Beko onto two dimensions with green plus signs over $\mathrm{RE}_{1}$ and $\mathrm{RE}_{2}$ in Fig. 3.13(a) and Fig. 3.13(b), respectively. As we see in Fig. 3.5(b) and Fig. 3.13(a)-3.13(b), $M$-Beko is an irregular constellation that does not fall on a grid. This can result in a more complex detection process.

\footnotetext{
${ }^{5}$ This constellation is available for different dimensions at the posted URL in [68].
} 


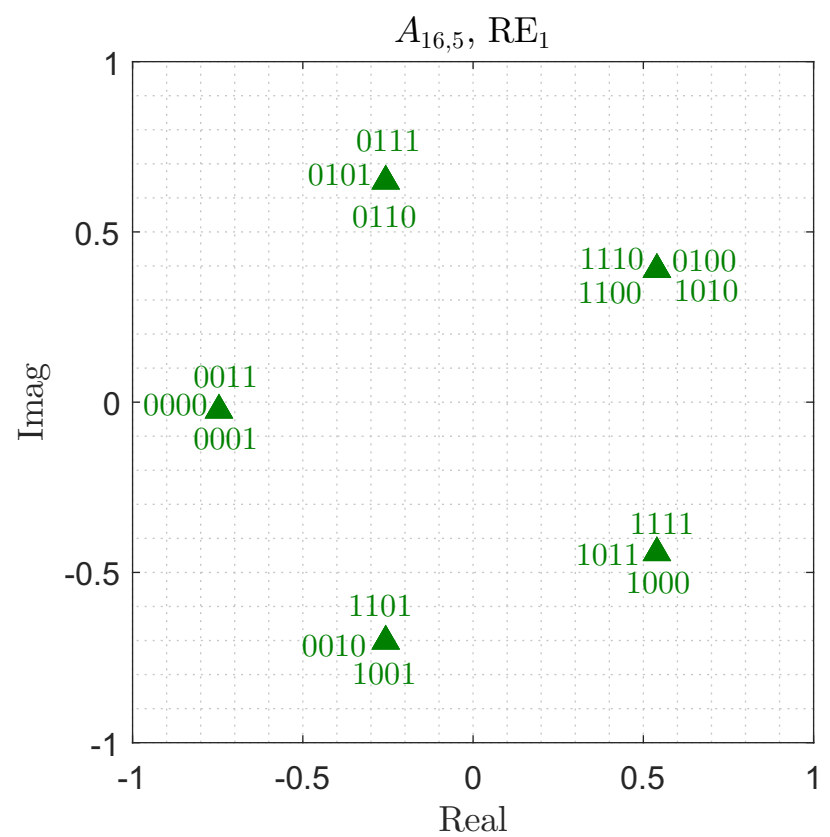

(a)

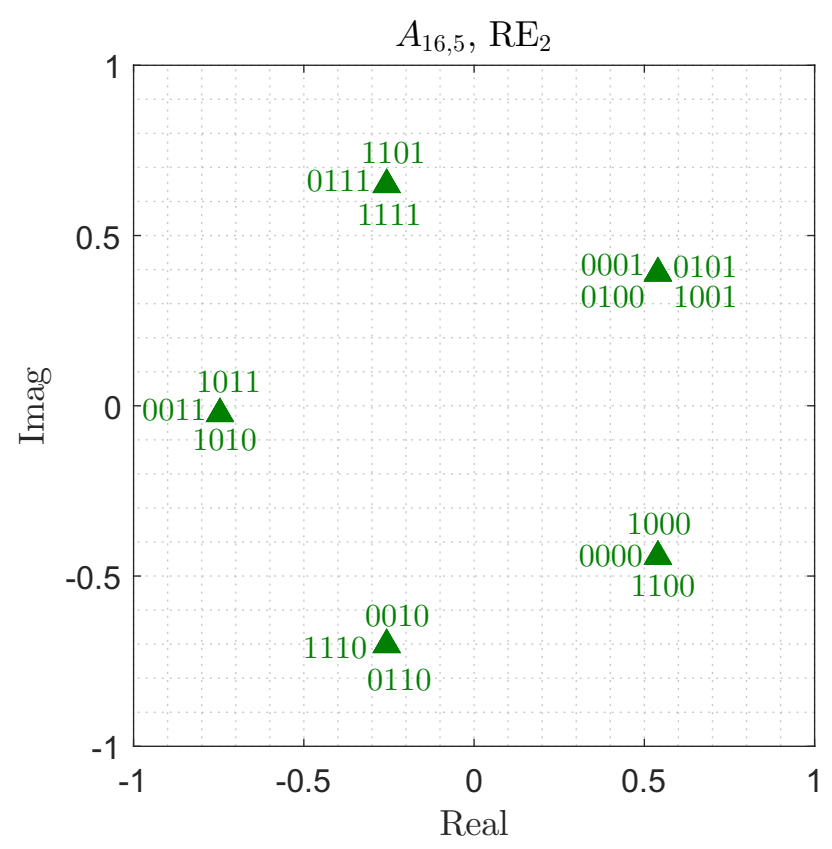

(b)

Figure 3.12: The projection of the $A_{16,5}$ constellation over (a) $R_{1}$, and (b) $R E_{2}$. 


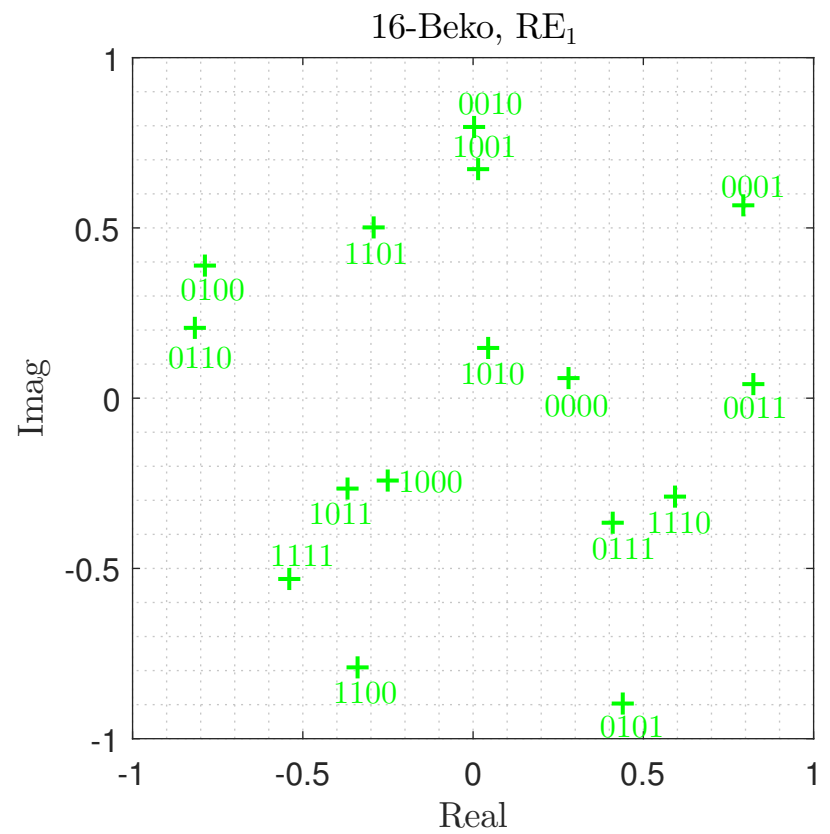

(a)

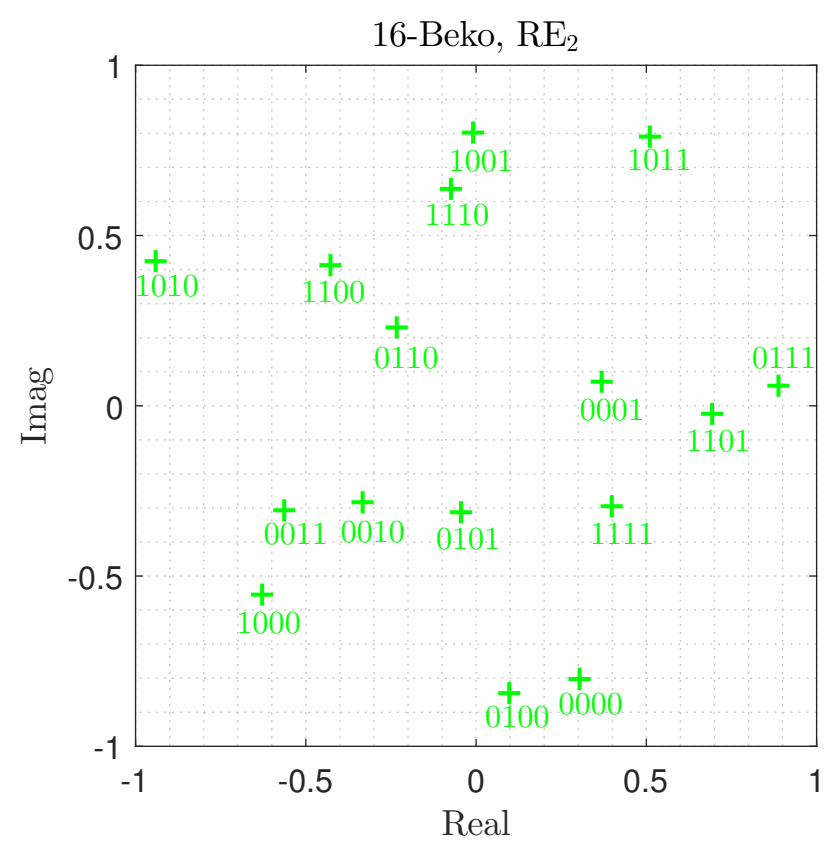

(b)

Figure 3.13: The projection of 16-Beko over (a) $\mathrm{RE}_{1}$, (b) $\mathrm{RE}_{2}$. 


\section{$M$-Peng}

An $M$-point codebook that is proposed for uncoded SCMA systems over AWGN channels in [62], and we refer to it as $M$-Peng. Unlike most of the constellations proposed so far in the context of SCMA systems, $M$-Peng is not based on a mother constellation that is common to all users, but instead is designed by maximizing $d_{E \text {, min }}$ between constellation points of all users for a power constraint. The design of this constellation is formulated as a non-convex optimization problem that is tackled by using the semidefinite relaxation (SDR) [92] technique.

For a scenario with $K=3$ users and $d_{v}=2$, we depict the 4-Peng constellation over $\mathrm{RE}_{1}$ and $\mathrm{RE}_{2}$ in Fig. 3.14(a) and Fig. 3.14(b), respectively. As we see in Fig. 3.14(a)3.14(b), similar to $M$-Beko, $M$-Peng is an irregular constellation, which can lead to a more complex detection process.

In [63], an $\boldsymbol{M}$-point constellation is proposed using a genetic algorithm for uncoded SCMA systems over AWGN channels. The constellation in [63] aims to maximize $d_{E}$, min between the constellation points for a fixed average energy. This constellation results in two pairs of antipodal codewords, that are then rotated by a 2-dimensional user-specific rotations. Once again, the impact of those user-specific rotations will disappear in the uplink fading scenarios. It is also shown in [63] that the proposed constellation does not perform well in fading scenarios.

\section{$M$-PSCMA}

The $M$-point permutation-based SCMA that which we name as $M$-PSCMA, is proposed in [64] for uncoded SCMA systems. A similar idea was originally employed in the context of CDMA and OFDM in [93-95]. Unlike the conventional SCMA systems, each PSCMA user is not assigned to a fixed user-to-RE mapping matrix, $\mathbf{F}_{k}$; the position of the non-zero elements in the user-to-RE mapping matrix for each PSCMA user is based on the permutation that is a function of the transmitted bit streams. In other words, the codebook of each user changes with the incoming bits, and therefore MPA cannot be used as the detection algorithm. A low complexity detector is then developed based on an iterative decoding algorithm in [96].

For a scenario with $K=6, N=4$, and $d_{v}=2$, we depict the 4-PSCMA constellation in Fig. 3.16. All users have an access to the all the available REs. However, they 


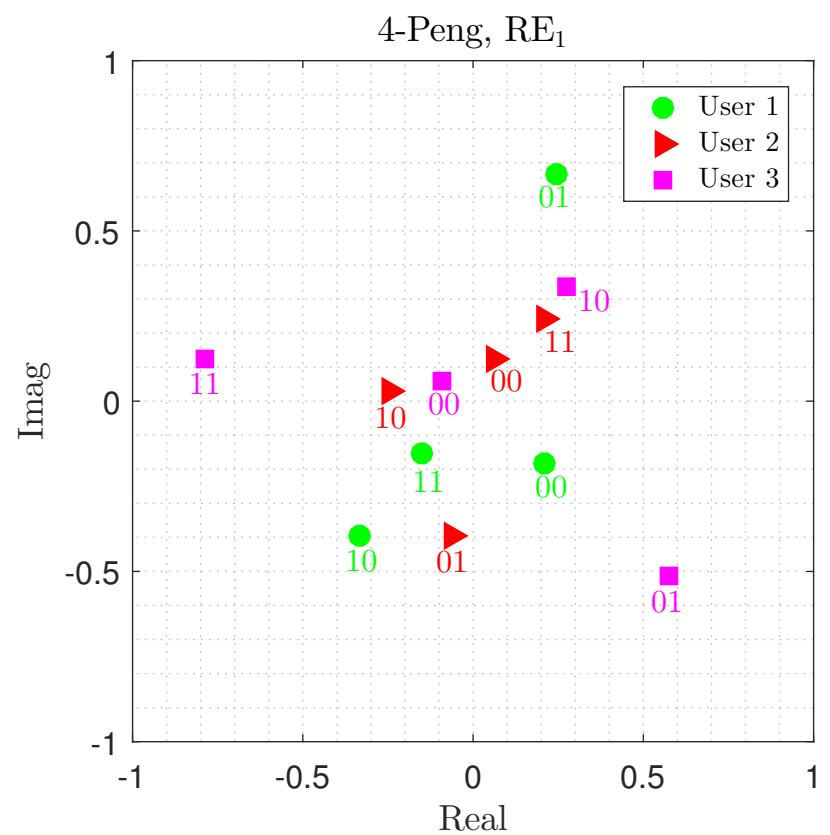

(a)

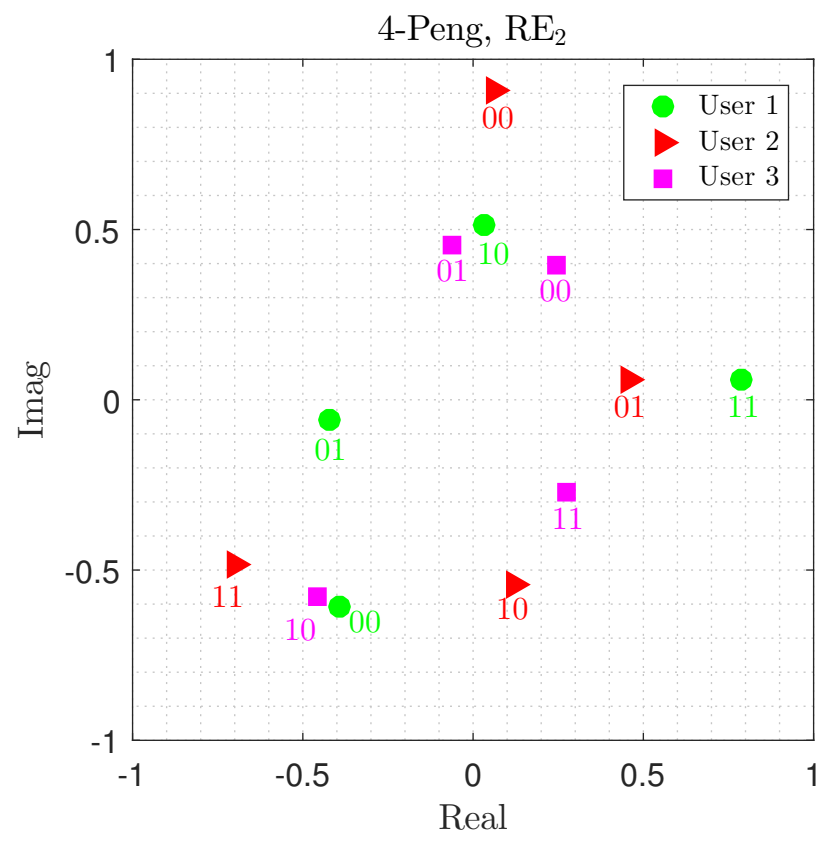

(b)

Figure 3.14: The projection of 4-Peng over (a) $\mathrm{RE}_{1}$, (b) $\mathrm{RE}_{2}$. 


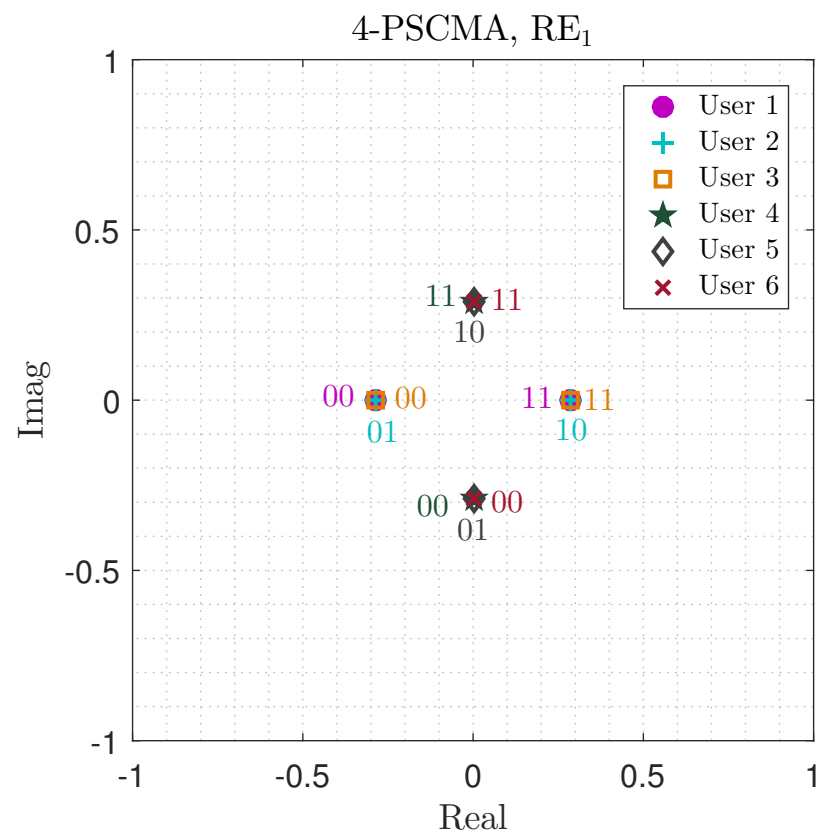

(a)

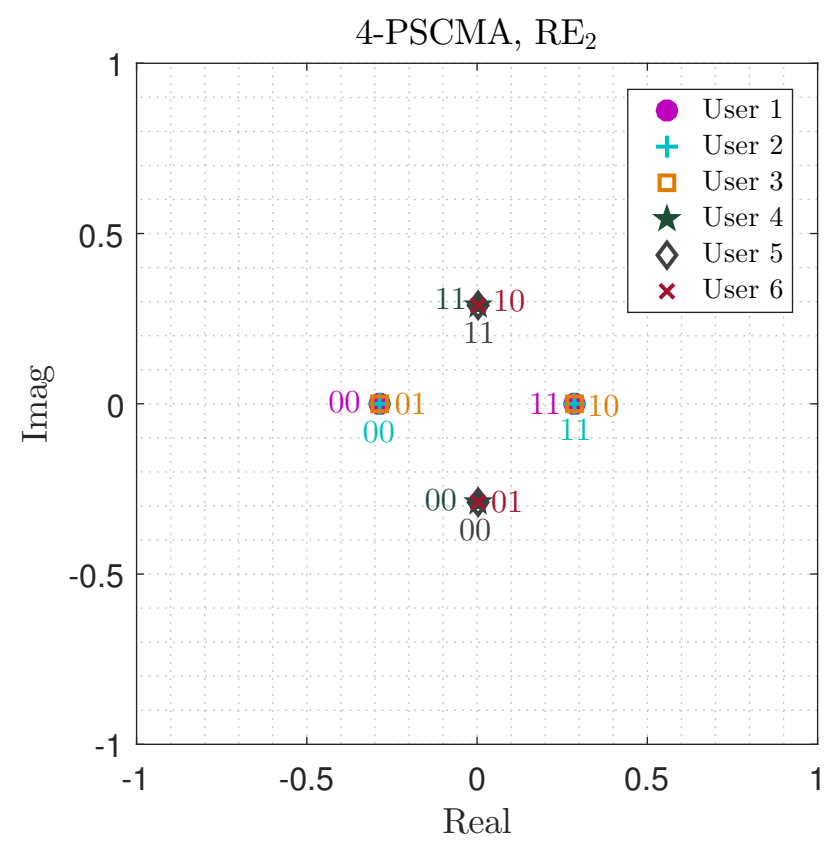

(b)

Figure 3.15: The projection of 4-PSCMA over (a) $\mathrm{RE}_{1}$, and (b) $\mathrm{RE}_{2}$. 


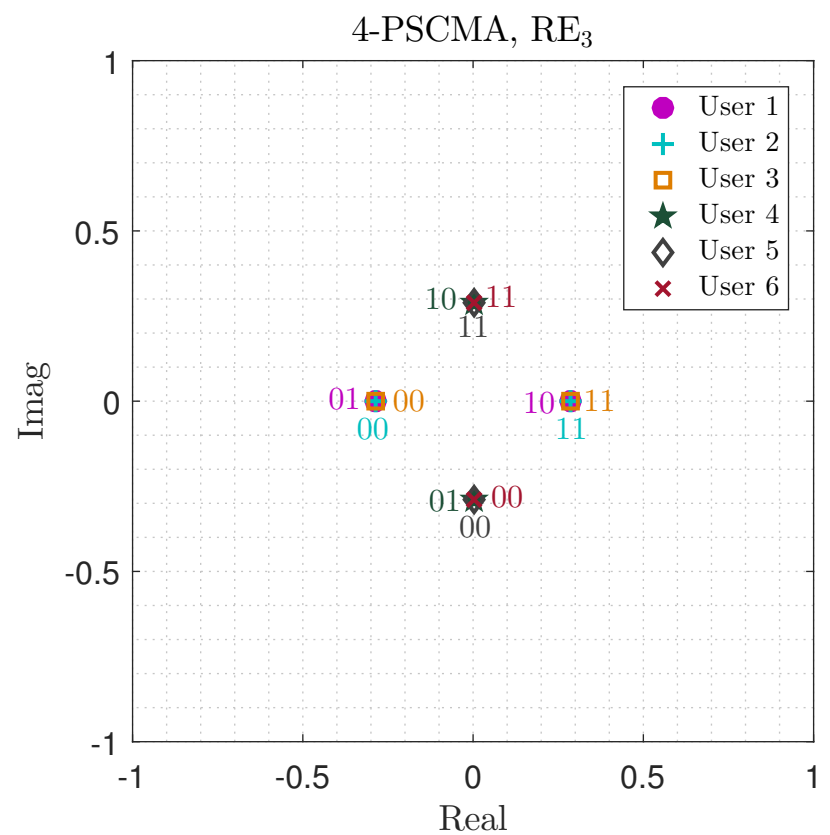

(a)

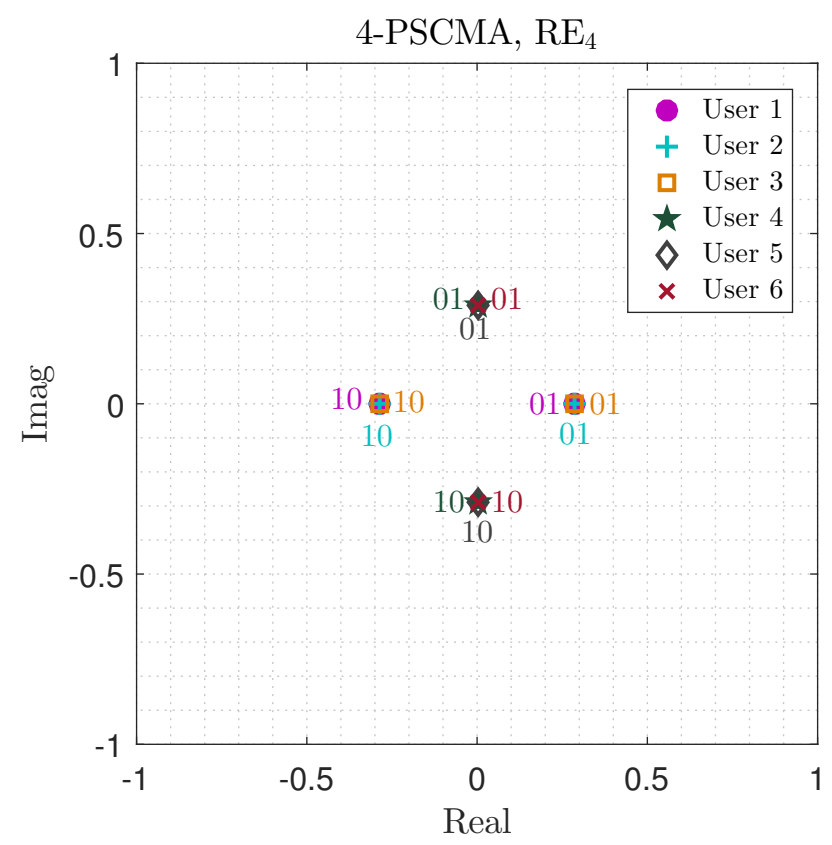

(b)

Figure 3.16: The projection of 4-PSCMA over (a) $\mathrm{RE}_{3}$, and (b) $\mathrm{RE}_{4}$. 
choose 2 out of the 4 REs according to their incoming bit streams. For instance, User 1 employs $R_{1}$ and $R_{2}$ to send 00 and 11, while it employs $R_{3}$ and $R_{4}$ to send 01 and 10. User 2 employs $\mathrm{RE}_{2}$ and $\mathrm{RE}_{3}$ to send 00 and 11 , while it employs $\mathrm{RE}_{1}$ and $\mathrm{RE}_{4}$ to send 01 and 10 . User 3 employs $\mathrm{RE}_{1}$ and $\mathrm{RE}_{3}$ to send 00 and 11 , while it employs $\mathrm{RE}_{2}$ and $\mathrm{RE}_{4}$ to send 01 and 10. The codebook of User 4 is the same as the codebook of User 1 except it is rotated by a phase shift of $\pi / 2$. Similarly, the codebooks of User 5 and User 6 are the same as the codebook of User 2 and User 3, respectively, but after a rotation of $\pi / 2$. As mentioned before, the $\pi / 2$ phase shift loses its importance in the uplink scenarios.

\subsection{Performance Evaluation}

In this section, we first provide the KPIs (Section 3.3) of an important subset ${ }^{6}$ of the constellations described in Section 3.4, namely, $M$-LDS, TMQAM, $M$ LQAM, $M$ Bao, 16HQAM, MCQAM, and $M$-Beko. We then evaluate the performance of those constellations over the different channel models presented in Section 2.1 through extensive MATLAB simulations. For convenience, in our all performance evaluation curves, we stick with the same color/line specification as in Fig. 3.3-Fig. 3.11 and Fig. 3.13. That is, the $M$-LDS constellation is represented by magenta left-pointing triangles, TMQAM by red circles, $M$ LQAM by blue asterisks, $M$-Bao by cyan squares, 16HQAM by brown crosses, $M$ CQAM by black points, and $M$-Beko by green plus signs.

\subsubsection{Constellations from the Standpoint of KPIs}

As discussed in Section 3.3, the KPIs of $M$-point $d_{v}$-dimensional complex constellations over different channel scenarios under study are as follows: The minimum Euclidean distance, $d_{E \text {, min }}$, the Euclidean kissing number, $\tau_{E}$, the minimum product distance, $d_{P \text {, min }}$, the product kissing number, $\tau_{P}$, the modulation diversity order, $L$,

\footnotetext{
${ }^{6}$ In our simulations, we did not assess the performance of the $M$-Peng, $A_{N_{m}, N_{d}}$, and $M$-PSCMA constellations due to the following reasons: The design of the $M$-Peng constellation depends on $K$ and $N$ [62], and we did not have access to this constellation designed for our system parameters. The $A_{N_{m}, N_{d}}$ constellation is designed specifically for BICM with iterative decoding and detection [59]. However, we use non-iterative detection and decoding in our simulations. Also, the M-PSCMA constellation requires a special receiver [64], and to maintain focus, we only use MPA with noniterative detection and decoding.
} 
Table 3.2: KPIs of 4-point constellations

\begin{tabular}{cccccccc}
\hline & $d_{E, \min }^{2}$ & $\tau_{E}$ & $d_{P, \min }^{2}$ & $\tau_{P}$ & $L$ & $N_{d}$ & Gray-labeled \\
\hline T4QAM & 2 & 2 & 0.64 & 2 & 2 & 4 & Yes \\
4LQAM & 2 & 2 & 2 & 2 & 1 & 2 & Yes \\
4CQAM & 2 & 2 & 1 & 2 & 1 & 3 & Yes \\
4-Beko & $\cong 2.67$ & 3 & $\cong 0.29$ & $1 / 2$ & 2 & 4 & No \\
4-Bao & 2 & 2 & $\cong 1$ & 2 & 2 & 4 & Yes \\
4-LDS & 2 & 2 & 1 & 2 & 2 & 4 & Yes \\
\hline
\end{tabular}

Table 3.3: KPIs of 16-point constellations

\begin{tabular}{cccccccc}
\hline & $d_{E, \min }^{2}$ & $\tau_{E}$ & $d_{P, \min }^{2}$ & $\tau_{P}$ & $L$ & $N_{d}$ & Gray-labeled \\
\hline T16QAM & 1 & 4 & 0.16 & 4 & 2 & 16 & Yes \\
16LQAM & 1 & 4 & 0.25 & 4 & 1 & 9 & Yes \\
16HQAM & 1 & 4 & 1 & 8 & 1 & 4 & Yes \\
16CQAM & $\cong 1.03$ & 2 & $\cong 0.21$ & 2 & 1 & 9 & No \\
16-Beko & $\cong 1.30$ & 7.75 & $\cong 0.02$ & $1 / 8$ & 2 & 16 & No \\
16-Bao & 1 & 4 & 0.25 & 4 & 2 & 16 & Yes \\
16-LDS & 0.4 & 3 & 0.04 & 3 & 2 & 16 & Yes \\
\hline
\end{tabular}

the number of distinct points, $N_{d}$, and whether the constellation is Gray-labeled or not. We provide those properties of the 4-point and 16-point 2-dimensional complex constellations under study in Table 3.2 and Table 3.3, respectively. From Table 3.2 and Table 3.3, we observe the following:

- 4-Beko and 16-Beko have the highest $d_{E \text {, min }}$ compared with the other 4-point and 16-point constellations. This is due to the fact that these constellations are proposed for AWGN channels, wherein $d_{E \text {, min }}$ is a KPI, and has been explicitly considered in the design process [68]. This notwithstanding, both 4-Beko and 
16-Beko also have the highest $\tau_{E}$ compared with other 4-point and 16-point constellations, which as we will discuss later, results in a performance degradation in some scenarios.

- From Section 3.4, there are number of overlaps among the projections of the constellation points in MLQAM, 16HQAM, and MCQAM. As such, the modulation diversity order of $M$ LQAM, $16 \mathrm{HQAM}$, and $M$ CQAM is $L=1$. Moreover, from (3.13), $N_{d}$ of the constellations are attained.

- As mentioned in Section 3.4, $M$-Bao is proposed for FIC scenarios. As $d_{P}$, min is a KPI in FIC, both 4-Bao and 16-Bao have the highest $d_{P \text {, min }}$ compared with other constellations with $L=2$.

- Due to the structure of $M$-Beko, it is not possible to use Gray labeling. Also, 16CQAM was not Gray-labeled when proposed in [55].

In what follows, we evaluate the performance of different constellations introduced in Section 3.4 under different channel scenarios described in Section 2.1. As pointed out in Section 3.2 and elaborated in Appendix B, the 2-dimensional user-specific rotations that are independently applied on each RE assigned to each user, e.g., [65], lose their importance in uplink scenarios due to the 2-dimensional random rotations introduced by the channel. As such, we assume all users employ the same constellations as the mother constellations provided in Section 3.4. We set $K=6, N=4$, $d_{v}=2, d_{c}=3,{ }^{7}$ with a single antenna at all transmitters, and a single antenna at the receiver. We also use the user-to-RE spreading matrix provided in (2.2), set 3 MPA iterations for the 4-point constellations, and $5 \mathrm{MPA}$ iterations for the 16-point constellations.

\subsubsection{Uncoded Scenarios}

In this section, we assess the SER and the BER performance of 4-point and 16-point 2-dimensional complex constellations with respect to SNR, that is defined as in (3.15), over FSC (Case 1) and FIC (Case 2). We define SNR as the average energy per bit

\footnotetext{
${ }^{7}$ Note that this has been the most common set up so far in different contexts of SCMA systems e.g., [29, 30, 55-57, 81, 82, 84, 97$]$.
} 
of the constellation divided by the noise variance. That is,

$$
\begin{aligned}
\mathrm{SNR} & =\frac{E_{\mathrm{S}}}{L_{M} N_{0}} \\
& =\frac{E_{\mathrm{b}}}{N_{0}},
\end{aligned}
$$

where $E_{\mathrm{b}}$ denotes the average energy per uncoded bit of the constellation, as $E_{\mathrm{S}}$ is given by (3.6).

\section{SER performance}

In Fig. 3.17-3.18, we compare the SER performance of uncoded SCMA systems with different 4-point and 16-point constellations over FSC channels (Case 1). As discussed in Section 3.3, the number of distinct points, $N_{d}$, the minimum Euclidean distance, $d_{E \text {,min }}$, and the Euclidean kissing number, $\tau_{E}$, are the KPIs for the FSC scenario. Since in FSC, each user observes the same channel coefficients over the $d_{v}=2$ REs, we expect that the channel diversity order to be 1 , i.e., a change of $10 \mathrm{~dB}$ in $E_{\mathrm{b}} / N_{0}$ per a change of one decade in SER [98].

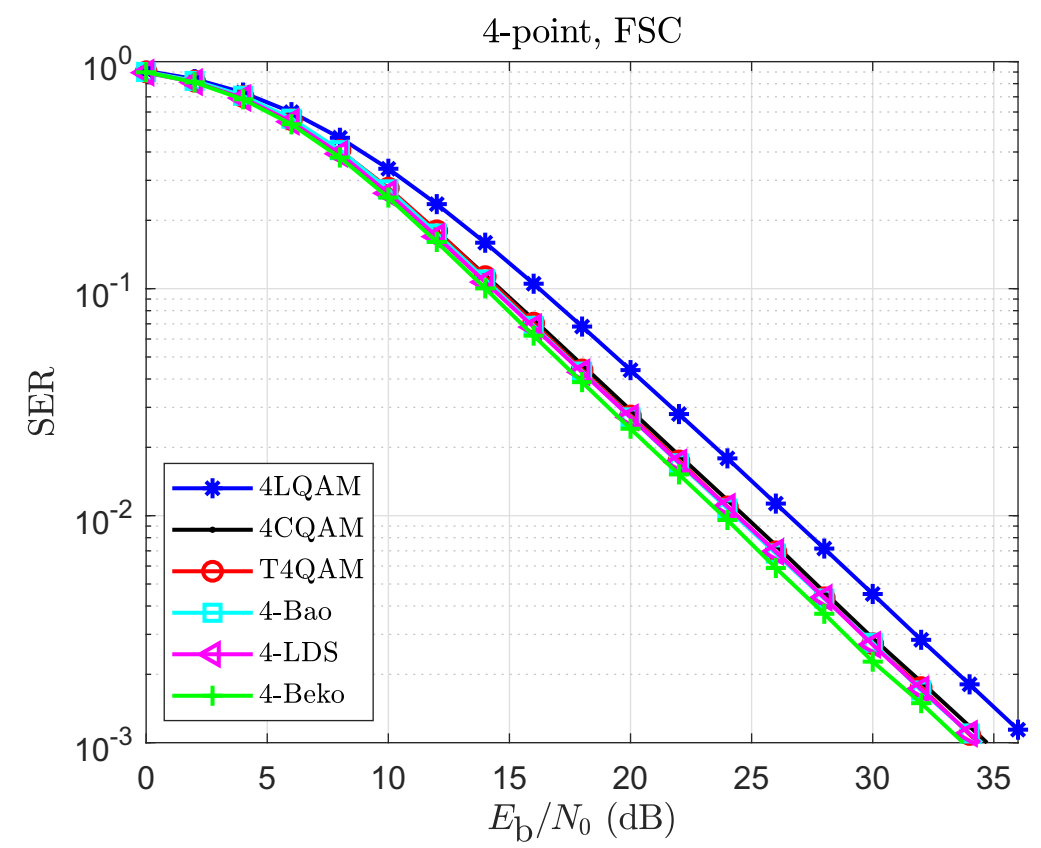

Figure 3.17: SER performance of uncoded SCMA systems with 4-point constellations over FSC (Case 1). 


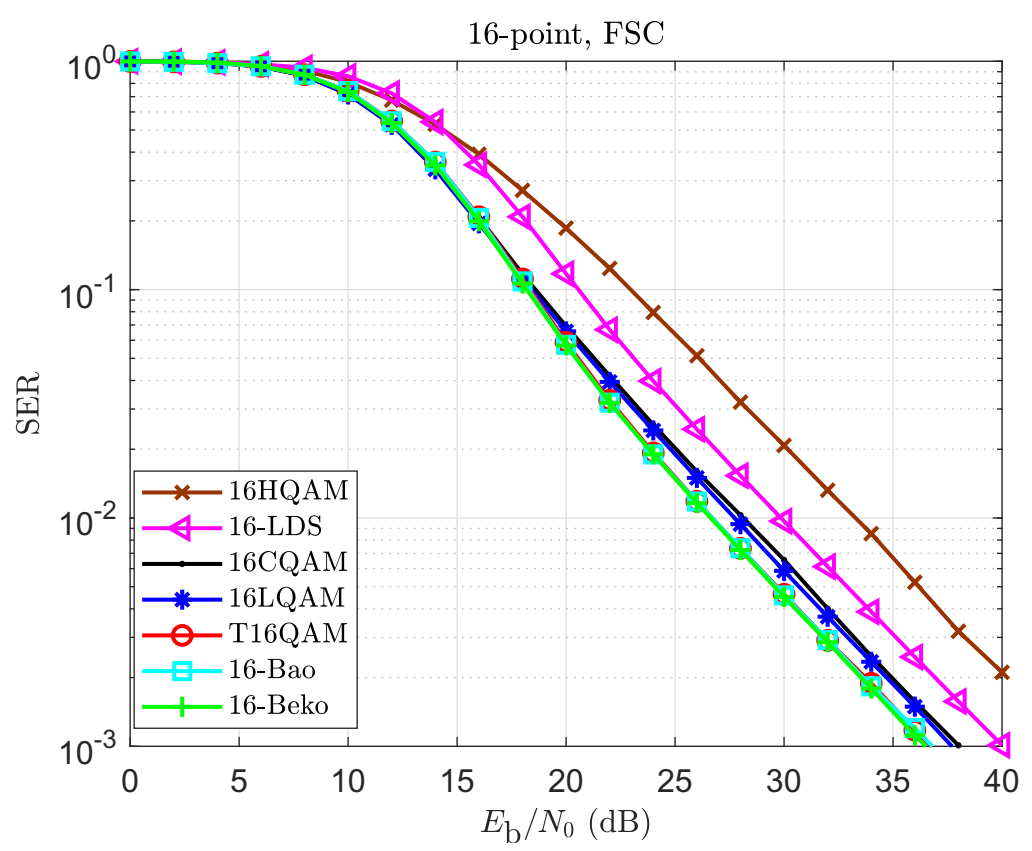

Figure 3.18: SER performance of uncoded SCMA systems with 16-point constellations over FSC (Case 1).

As shown in Fig. 3.17, we note that amongst the 4-point constellations, the 4Beko constellation (Section 3.4), which is carefully designed for the AWGN in [68], performs the best in this scenario. As from Table 3.2, 4-Beko (Section 3.4) has $N_{d}=4$, the highest $d_{E \text {,min }}$, and a comparable $\tau_{E}$ with other 4-point constellations. Moreover, from Table 3.2, since 4-Bao (Section 3.4), 4-LDS (Section 3.4), and T4QAM (Section 3.4) have $N_{d}=4$ and the same $d_{E \text {,min }}$ and $\tau_{E}$, they behave similarly in FSC. However, 4CQAM (Section 3.4) performs slightly worse due to its $N_{d}=3$. We also observe that 4LQAM (Section 3.4) has the lowest $N_{d}=2$, and thus falls behind the other constellations in this scenario.

Amidst the 16-point constellations, we notice that 16-Beko (Section 3.4), T16QAM (Section 3.4), and 16-Bao (Section 3.4) outperform the other constellations in Fig. 3.18. Referring to Table 3.3, 16-Beko, T16QAM, 16-Bao, and 16-LDS (Section 3.4) have an $N_{d}$ of 16. However, we observe in Fig. 3.18 that 16-LDS lags behind 16-Beko, T16QAM, and 16-Bao due to its lower $d_{E \text {,min }}$. Further, although 16-Beko has the highest $d_{E \text {,min }}$ compared with the others, it is also with the highest $\tau_{E}$. As such, it performs similar to T16QAM and 16-Bao (which have the same $d_{E \text {,min }}$ and $\tau_{E}$ ). We also note that 16HQAM (Section 3.4) has the lowest $N_{d}=4$, and thus it stays behind all the other constellations in this scenario. 


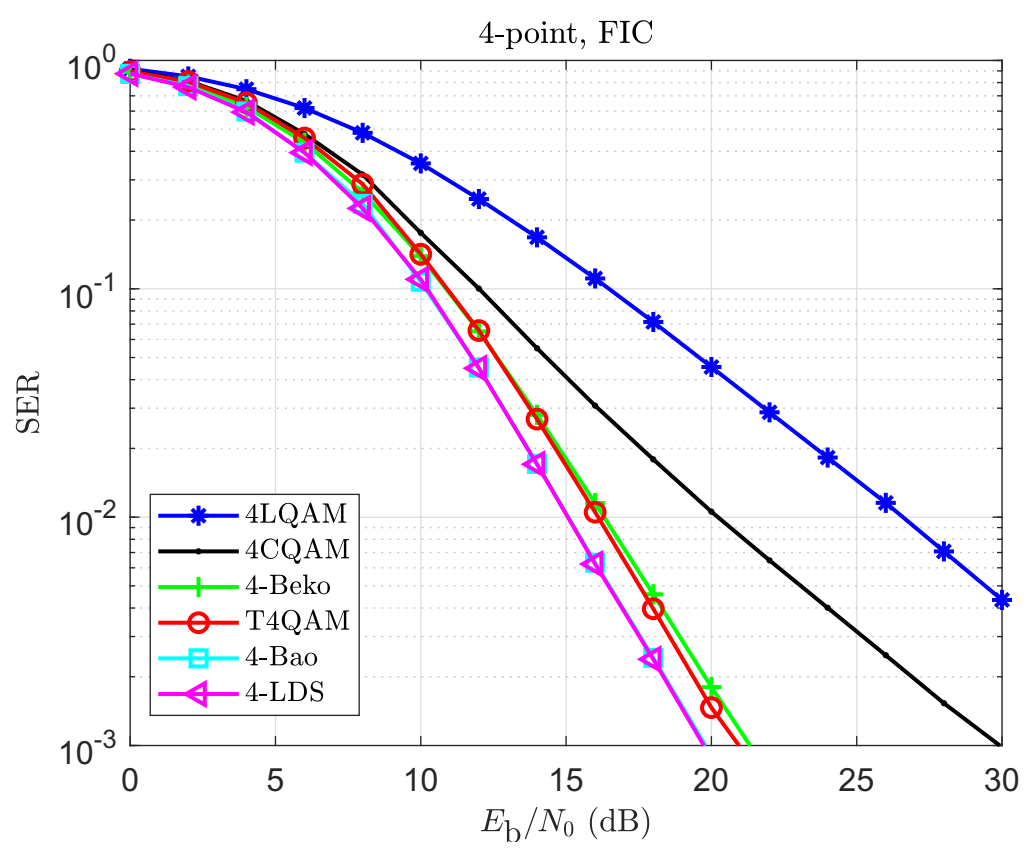

Figure 3.19: SER performance of uncoded SCMA systems with 4-point constellations over FIC (Case 2).

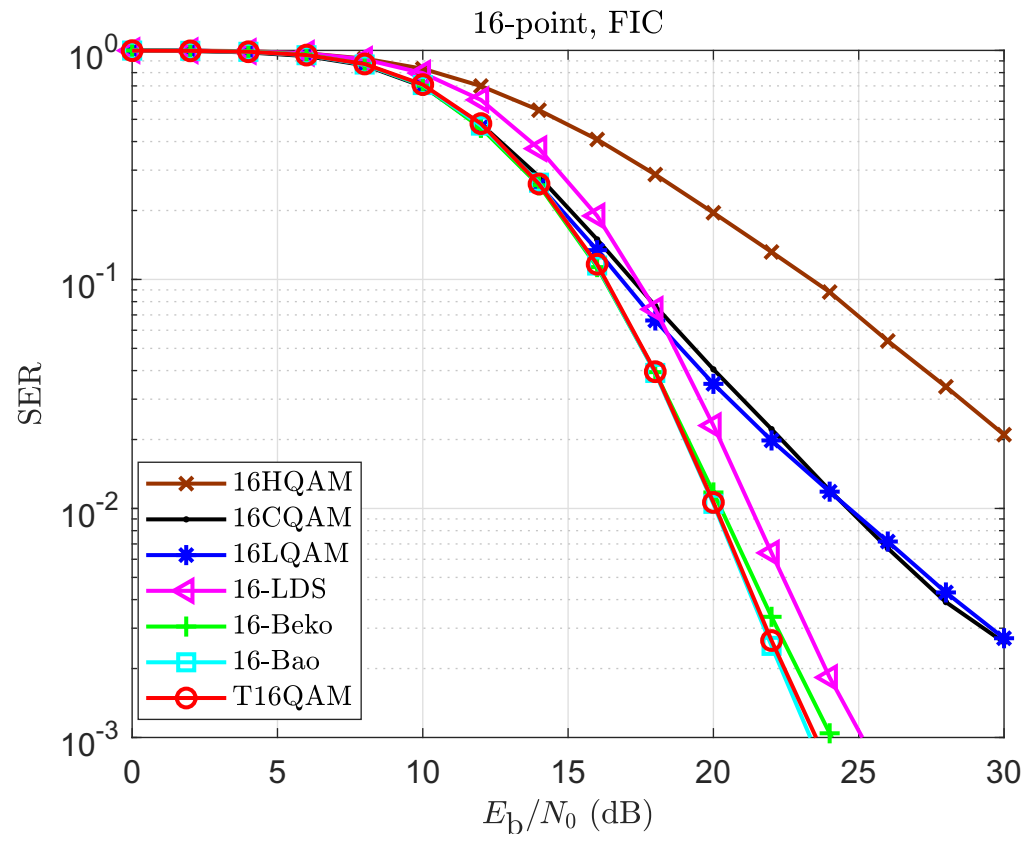

Figure 3.20: SER performance of uncoded SCMA systems with 16-point constellations over FIC (Case 2). 
We depict the SER performance of uncoded SCMA systems with different 4-point and 16-point constellations over FIC channels (Case 2) in Fig. 3.19 and Fig. 3.20, respectively. As addressed in Section 3.3, the number of distinct points, $N_{d}$, the modulation diversity order, $L$, the minimum product distance, $d_{P, \min }$, and the product kissing number, $\tau_{P}$, are the KPIs for the FIC scenario. Since in FIC, each user observes different channel coefficients over the $d_{v}=2$ REs, the channel diversity order is 2 , i.e., a change of $5 \mathrm{~dB}$ in $E_{\mathrm{b}} / N_{0}$ per a change of one decade in SER.

Fig. 3.19 shows the 4-point constellations in the FIC scenario. From Table 3.2, 4-Bao, 4-LDS, T4QAM, and 4-Beko have $L=2$ and $N_{d}=4$, and so perform similarly. Among these constellations, 4-Bao and 4-LDS have the highest $d_{P, \min }$ and the same $\tau_{P}$, and as such, they outperform the others. Moreover, compared with the constellations with $L=2$, the performance of 4 -Beko is degraded by its lowest $d_{P, \min }$. Also, since 4LQAM has the lowest $N_{d}$ and $L$ compared with the others, it lags behind them.

As Fig. 3.20 presents, amidst the 16-point constellations, 16-Bao beats the others over the FIC scenario. Referring to Table 3.3, 16-Bao, T16QAM, 16-Beko, and 16LDS have $L=2$ and $N_{d}=16$. Among these constellations, we see that 16-Bao has the highest $d_{P \text {, min }}$ and the same $\tau_{P}$ as T16QAM and 16-LDS. As such, it outperforms the others. Note that although 16-LDS has a slightly higher $d_{P \text {, min }}$ compared with 16-Beko, it still stays behind it due to its higher $\tau_{P}$. Further, 16HQAM performs very poorly in this scenario due its low $L$ and $N_{d}$.

\section{BER performance}

We compare the BER performance of uncoded SCMA systems introduced in Section 3.5.2, in Fig. 3.21-3.24. As mentioned in Section 3.3, in addition to the KPIs for the SER performance of FSC and FIC scenarios mentioned above, bit-labeling has a significant impact on the BER performance of uncoded systems. Moreover, the behavior of the multiuser detector at different SNRs affects the performance of the system. For the illustrative purpose, the BER performance of the uncoded systems at low SNRs is magnified in Fig. 3.21-3.24. We note that the presence of multiuser interference (MUI) changes the ordering of the BER curves at low SNRs. 


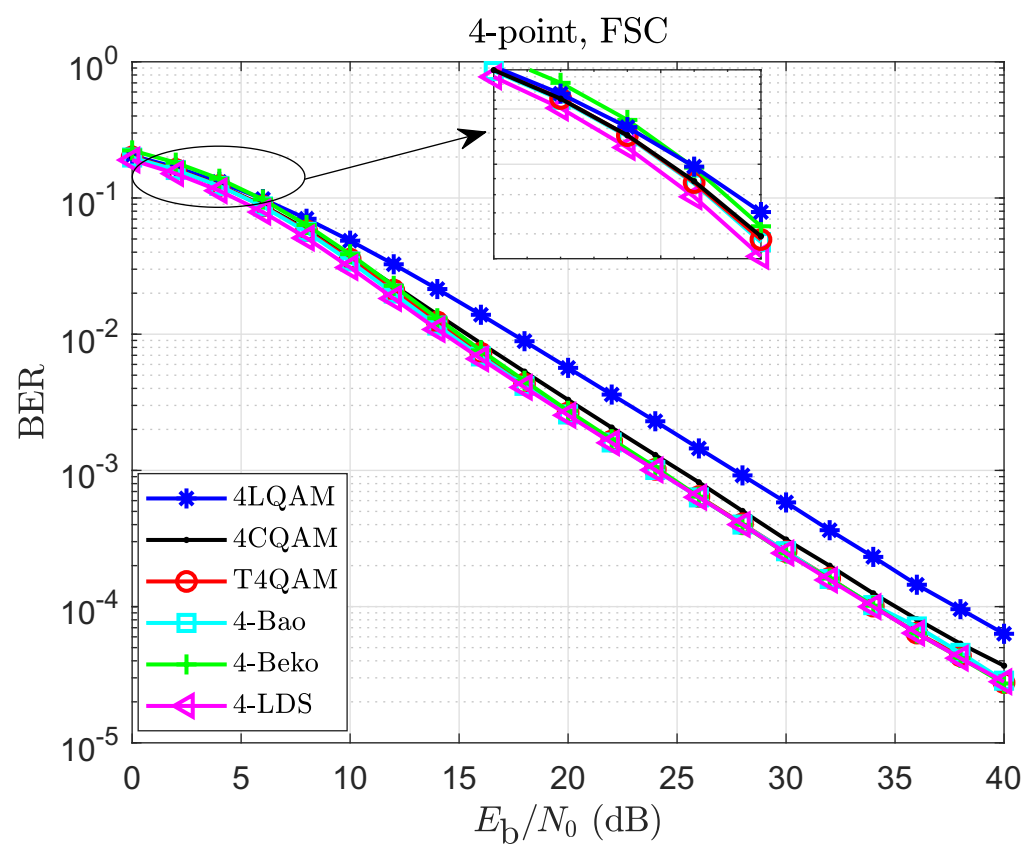

Figure 3.21: BER performance of uncoded SCMA systems with 4-point constellations over FSC (Case 1).

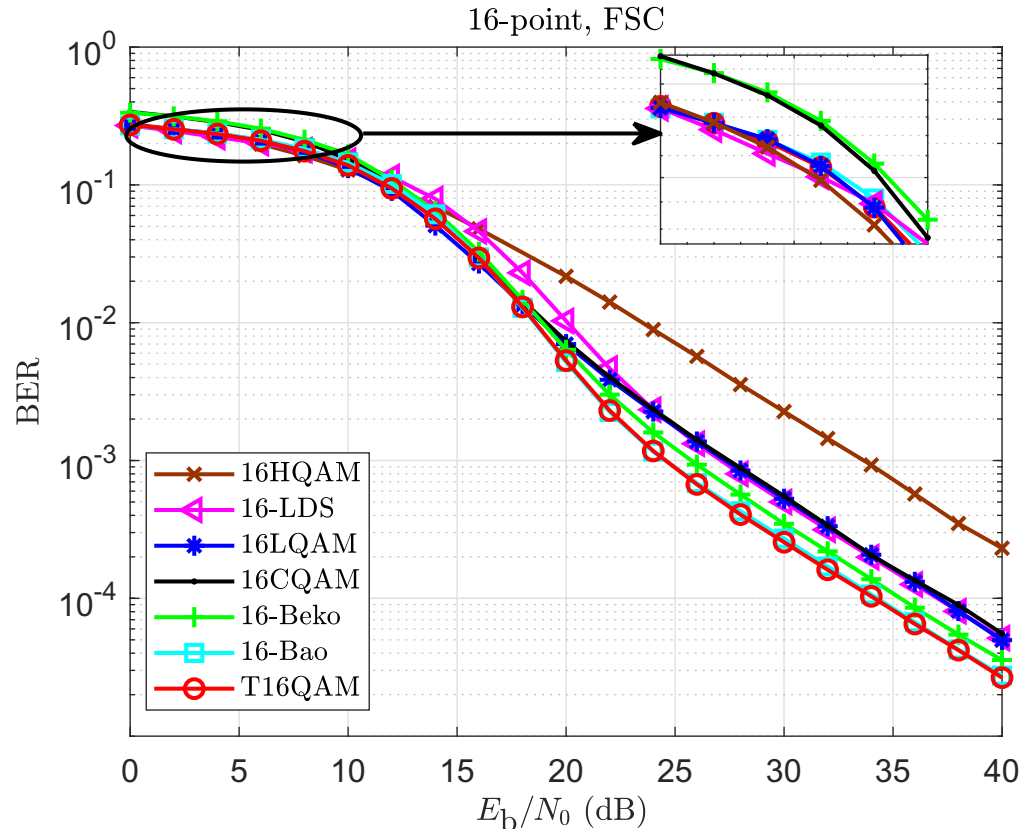

Figure 3.22: BER performance of uncoded SCMA systems with 16-point constellations over FSC (Case 1). 


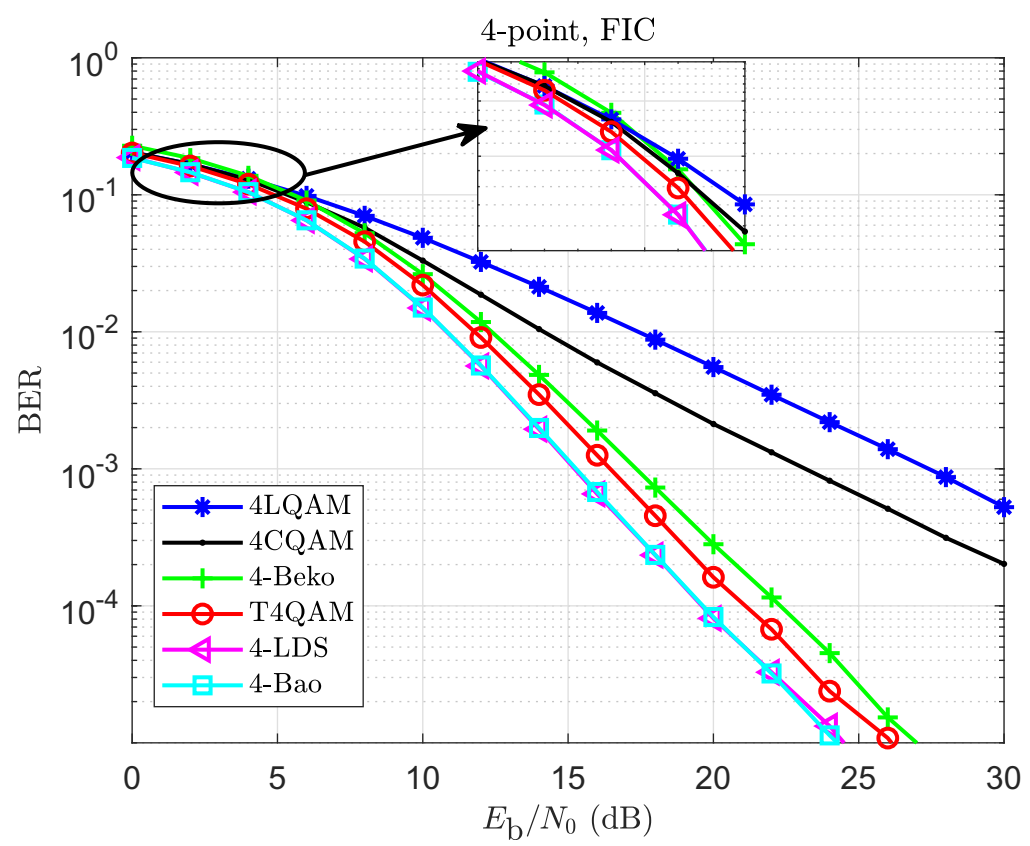

Figure 3.23: BER performance of uncoded SCMA systems with 4-point constellations over FIC (Case 1).

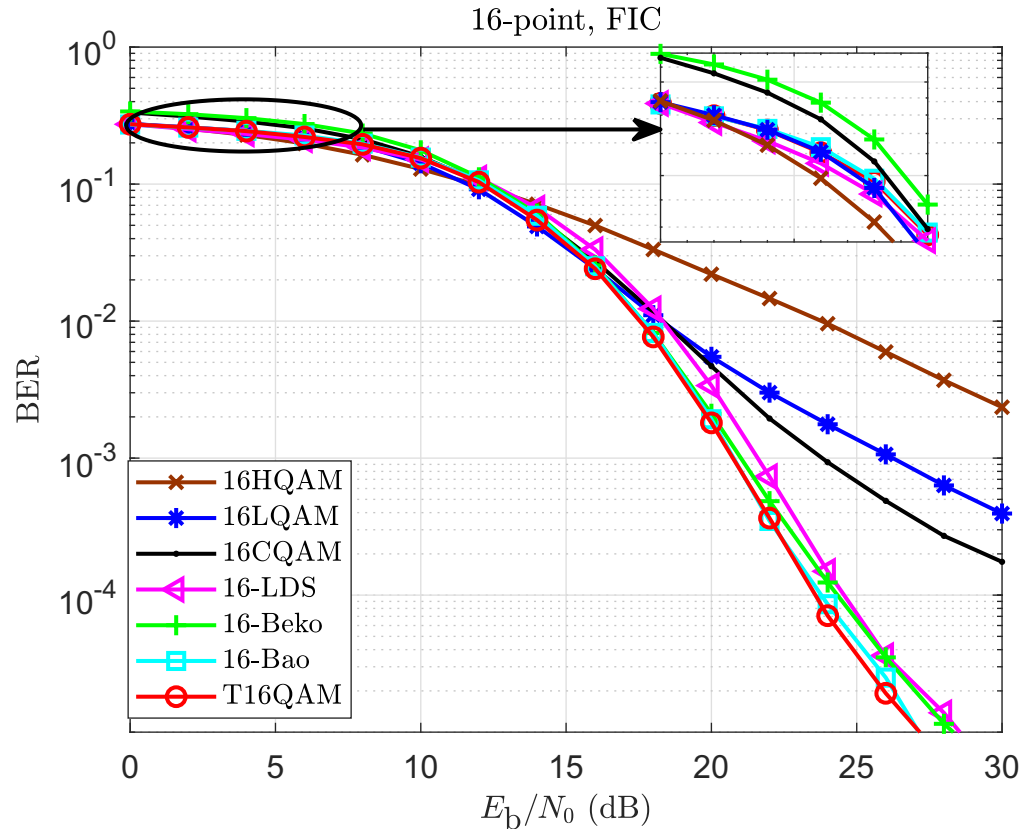

Figure 3.24: BER performance of uncoded SCMA systems with 16-point constellations over FIC (Case 1). 
From Table 3.2 and Table 3.3, $M$-Beko and 16CQAM constellations are not Graylabeled. Therefore, we observe in Fig. 3.21-3.24 that compared with their SER performance, $M$-Beko and 16CQAM perform differently with respect to other constellations. For instance, we see in Fig. 3.21 and Fig. 3.22 that unlike their SER performance in Fig. 3.17 and Fig. 3.18, 4-Beko and 16-Beko do not outperform the other constellations.

\subsubsection{High-Rate Turbo-coded Scenarios}

In this section, we evaluate the frame error rate (FER) performance of high-rate turbocoded SCMA systems with 4-point and 16-point 2-dimensional complex constellations with respect to SNR over FFSC (Case 3), FFIC (Case 4), SFSC (Case 5), and SFIC (Case 6). We define SNR as the average energy per message bit of the constellation divided by the noise variance. That is,

$$
\begin{aligned}
\mathrm{SNR} & =\frac{E_{\mathrm{S}}}{R L_{M} N_{0}} \\
& =\frac{E_{\mathrm{mb}}}{N_{0}},
\end{aligned}
$$

where $E_{\mathrm{mb}}$ denotes the average energy per bit of the constellation, and $R$ is the rate of the turbo code.

We employ an LTE turbo code [39] with a rate of $R=4 / 5$ and a codeword length of $N_{c}=120$. In our simulations, we use 4 turbo iterations. We use the turbo encoder followed by the rate-matcher used in the LTE standard in [39]. The LTE rate-matcher includes interleaving between the encoder and the signal mapper and is described in [39]. Interested readers can refer to [99] for more information about implementing the LTE turbo code at different rates.

Fig. 3.25-3.26 show the FER performance of turbo-coded SCMA systems with $R=4 / 5$ and different 4-point and 16-point constellations for the FFSC scenario. As discussed in Section 3.3, the type of bit-labeling, the SNR operating region, $N_{d}$, $d_{E \text {,min }}$, and $\tau_{E}$ are the KPIs for this scenario.

Amongst the 4-point constellations, we note that 4-LDS is the best choice in Fig. 3.25. It is worthwhile to note that 4-Bao lags behind 4-LDS in this scenario. This can be justified through the BER performance of uncoded systems at mid-range 


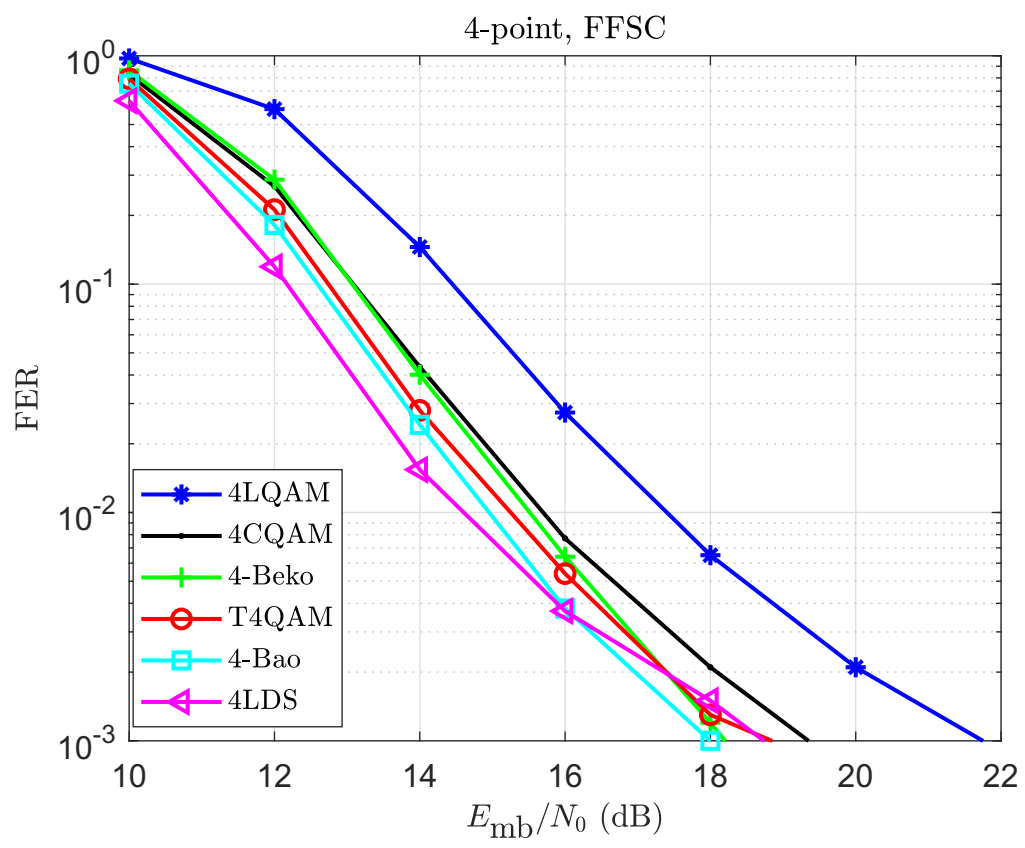

Figure 3.25: FER performance of $R=4 / 5$ turbo-coded SCMA systems with 4-point constellations over FFSC (Case 3).

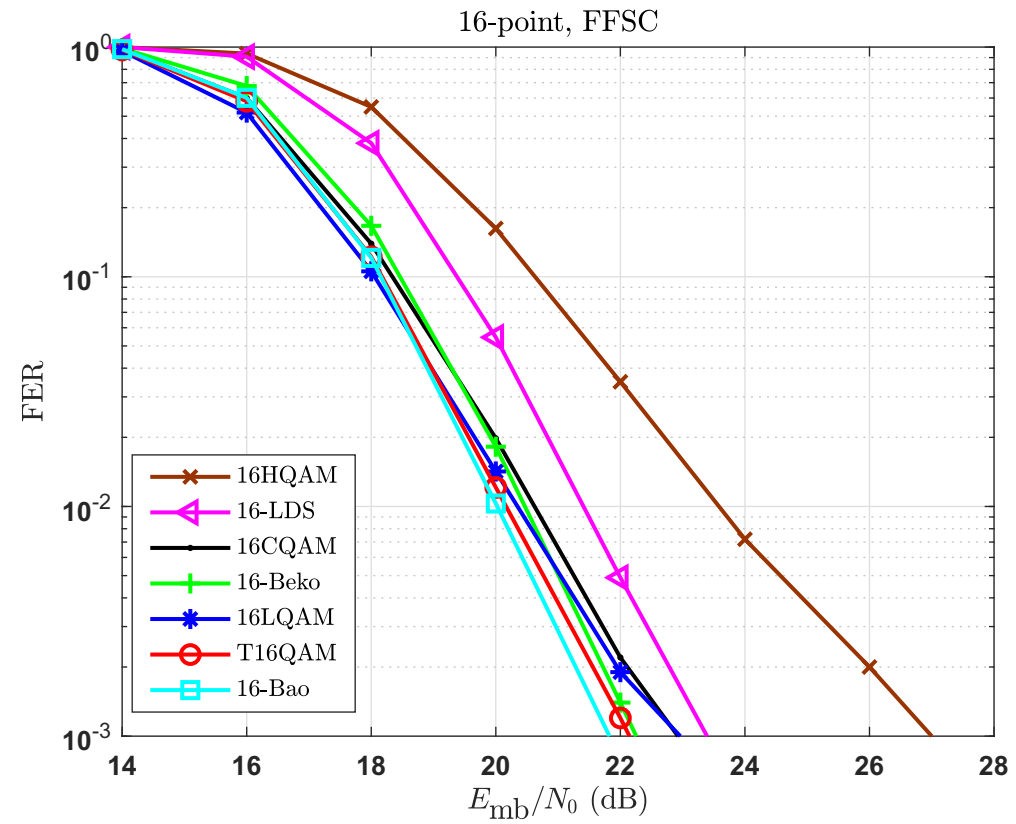

Figure 3.26: FER performance of $R=4 / 5$ turbo-coded SCMA systems with 16point constellations over FFSC (Case 3). 
SNRs in Fig. 3.21. ${ }^{8}$ Moreover, similar to the BER performance in uncoded system in Fig. 3.21, although from Table 3.2, 4-Beko has $N_{d}=4$, the highest $d_{E \text {,min }}$, and a comparable $\tau_{E}$ with the other 4-point constellations, it does not perform as well as 4LDS due to its bit-labeling. We also observe that 4LQAM has the lowest $N_{d}=2$, and thus falls behind the others. On the other hand, amidst the 16-point constellations, we notice that 16-Bao and T16QAM outperform the others in Fig. 3.26. Referring to Table 3.3, 16-Beko, T16QAM, 16-Bao, and 16-LDS have $N_{d}=16$. However, we observe in Fig. 3.26 that 16-LDS lags behind T16QAM, 16-Bao, 16-Beko due to its comparable $\tau_{E}$, and its lowest $d_{E \text {,min }}$. Further, although 16-Beko has the highest $d_{E \text {,min }}$, it also has the highest $\tau_{E}$, and is not Gray-labeled. As such, it lags behind 16Bao and T16QAM. We also note that similar to the FSC case (Fig. 3.18), 16HQAM has the lowest $N_{d}=4$, and thus it stays behind all the other constellations in this scenario.

We depict the FER performance of turbo-coded SCMA systems with $R=4 / 5$ and different 4-point and 16-point constellations over FFIC channels in Fig. 3.27 and Fig. 3.28, respectively. As addressed in Section 3.3, the type of bit-labeling, the SNR operating region, $N_{d}, L, d_{p, \min }$, and $\tau_{p}$ are the KPIs for the FFIC scenario.

As Fig. 3.27 shows, amongst the 4-point constellations, 4-LDS and 4-Bao outperform the others in the FFIC scenario. Following the similar illustration as in the FIC case (Fig. 3.19) and from Table 3.2, 4-Bao, 4-LDS, T4QAM, and 4-Beko have the highest $L$ with $N_{d}=4$. Among these constellations, 4-Bao and 4-LDS have the highest $d_{P, \min }$ and the same $\tau_{P}$. As such, both 4-Bao and 4-LDS outperform the others. Moreover, compared with the other constellations with $L=2$, the performance of 4Beko is deteriorated by its lowest $d_{P, \min }$ and its bit-labeling. Also, similar to the FIC case, since $4 \mathrm{LQAM}$ has the lowest $N_{d}$ and $L$, it lags behind the others. As Fig. 3.28 presents, amidst the 16-point constellations, 16-Bao and T16QAM beat the others over the FFIC scenario. As for the FIC case (Fig. 3.20), and by referring to Table 3.3, 16-Bao, T16QAM, 16-Beko, and 16-LDS have the highest $L$ with $N_{d}=16$. Among these, we see that 16 -Bao has the highest $d_{P, \min }$, a comparable $\tau_{P}$ with T16QAM and 16-LDS, and a similar bit-labeling. Moreover, 16HQAM performs very poorly in this scenario due its lowest $L$ and lowest $N_{d}$.

\footnotetext{
${ }^{8}$ Of note, the energy per message bit in Fig. 3.25 and the energy per uncoded bit in Fig. 3.21 are related by $10 \log (R)$.
} 


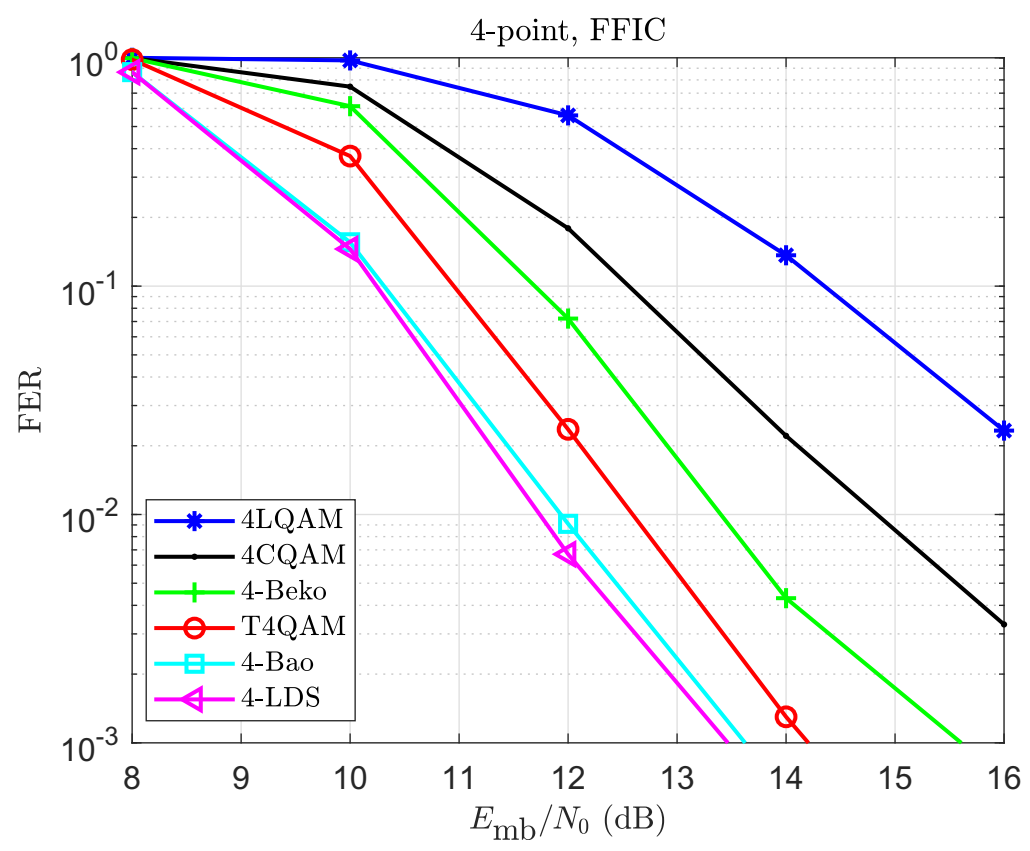

Figure 3.27: FER performance of $R=4 / 5$ turbo-coded SCMA systems with 4-point constellations over FFIC (Case 4).

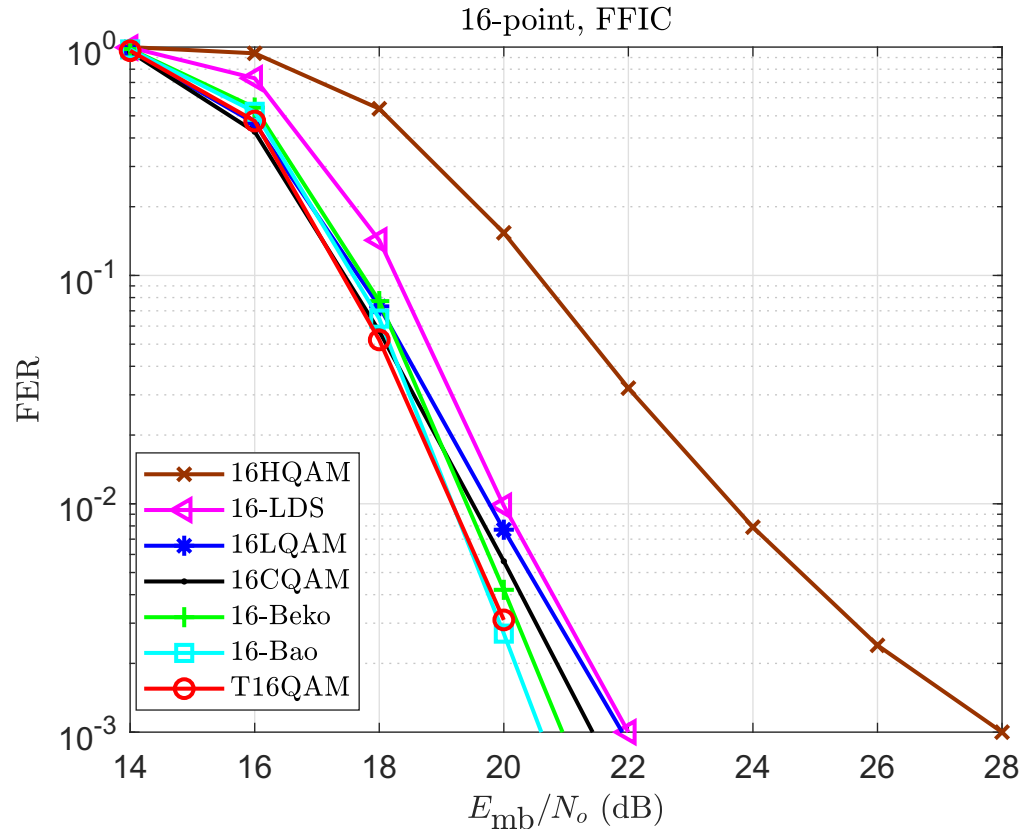

Figure 3.28: FER performance of $R=4 / 5$ turbo-coded SCMA systems with 16point constellations over FFIC (Case 4). 
In Fig. 3.29-3.32, we compare the FER performance of the systems described above over SFSC and SFIC channels. Note that since in slow quasi-static fading scenarios each channel coefficient is constant for the duration of transmission of the whole codeword, the system performs poorly. Hence, in slow quasi-static fading scenarios, higher SNR regions are mainly of interest.

Similar to the FFSC case, the type of bit-labeling, the SNR operating region, $N_{d}$, $d_{E \text {,min }}$, and $\tau_{E}$ are the KPIs in SFSC. Also, similar to the FFIC case, the type of bit-labeling, the SNR operating region, $N_{d}, L, d_{P, \min }$, and $\tau_{P}$ are the KPIs in SFIC.

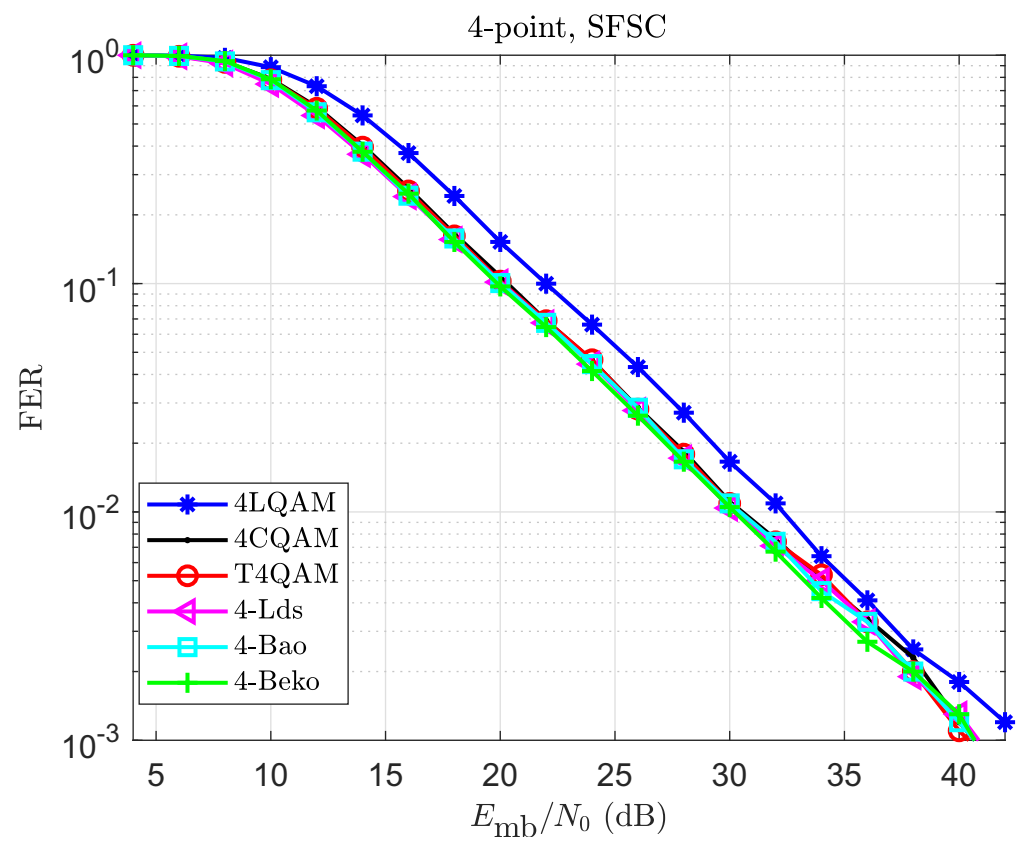

Figure 3.29: FER performance of $R=4 / 5$ turbo-coded SCMA systems with 4-point constellations over SFSC (Case 5).

From Fig. 3.29 and Fig. 3.30, we see that the trends of different 4-point and 16point constellations in SFSC are consistent with the trends of the BER performance of uncoded systems at high SNRs in the FSC case, i.e., Fig. 3.21 and Fig. 3.22. Further, from Fig. 3.31 and Fig. 3.32, we observe that the trends of different 4-point and 16point constellations in SFIC for a target FER of $10^{-2}$ are consistent with the trends of the BER performance of uncoded systems at their corresponding SNRs $(\cong 17.03$ $\mathrm{dB}$ and $\cong 20.03 \mathrm{~dB}$ in the 4 -Bao and 16-Bao cases, respectively) for the FIC case, 


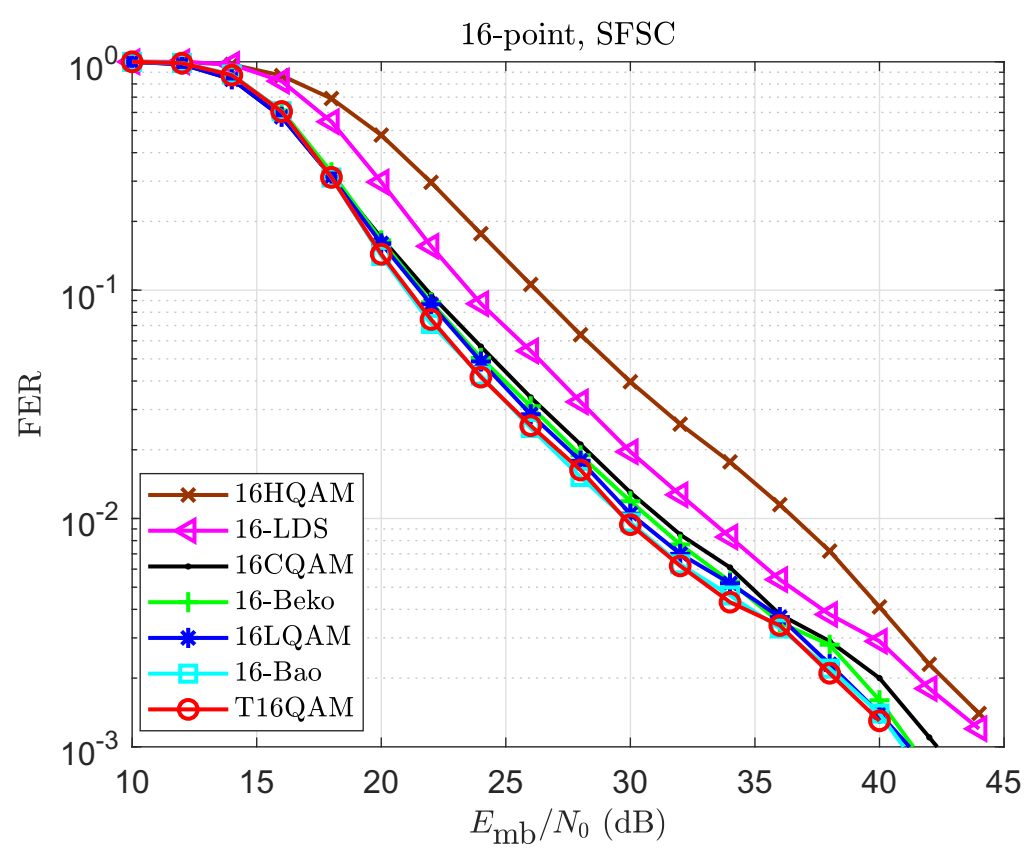

Figure 3.30: FER performance of $R=4 / 5$ turbo-coded SCMA systems with 16point constellations over SFSC (Case 5).

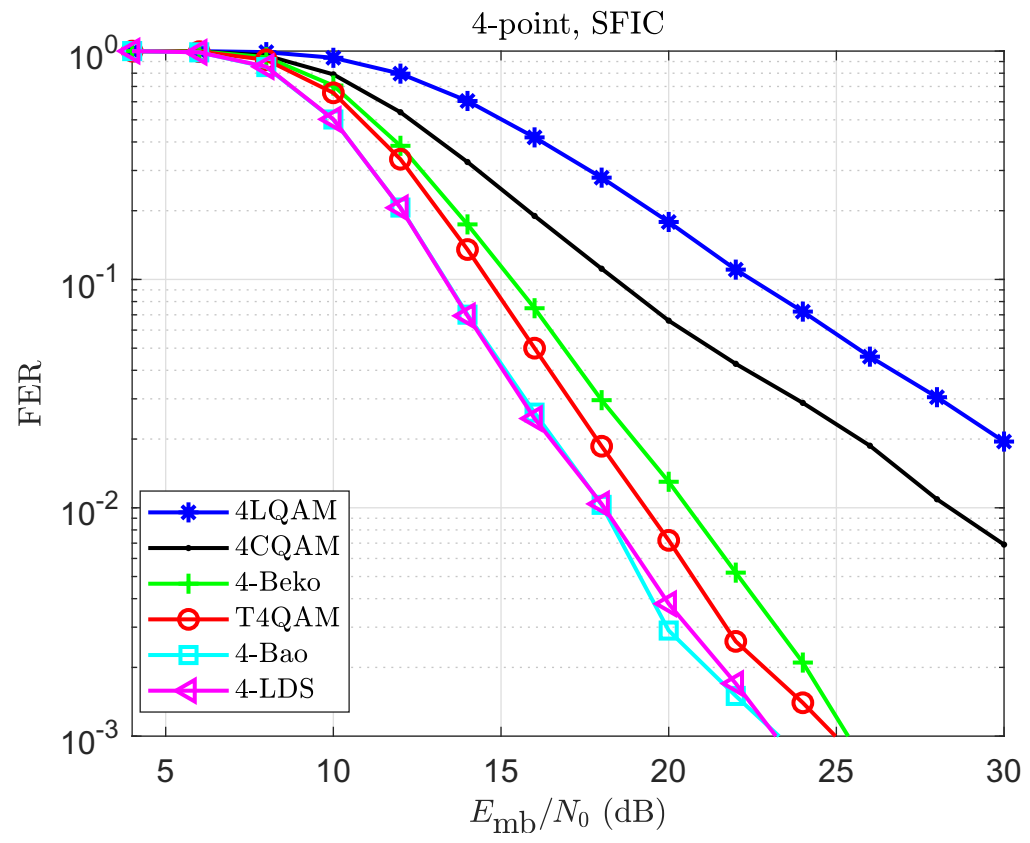

Figure 3.31: FER performance of $R=4 / 5$ turbo-coded SCMA systems with 4-point constellations over SFIC (Case 6). 


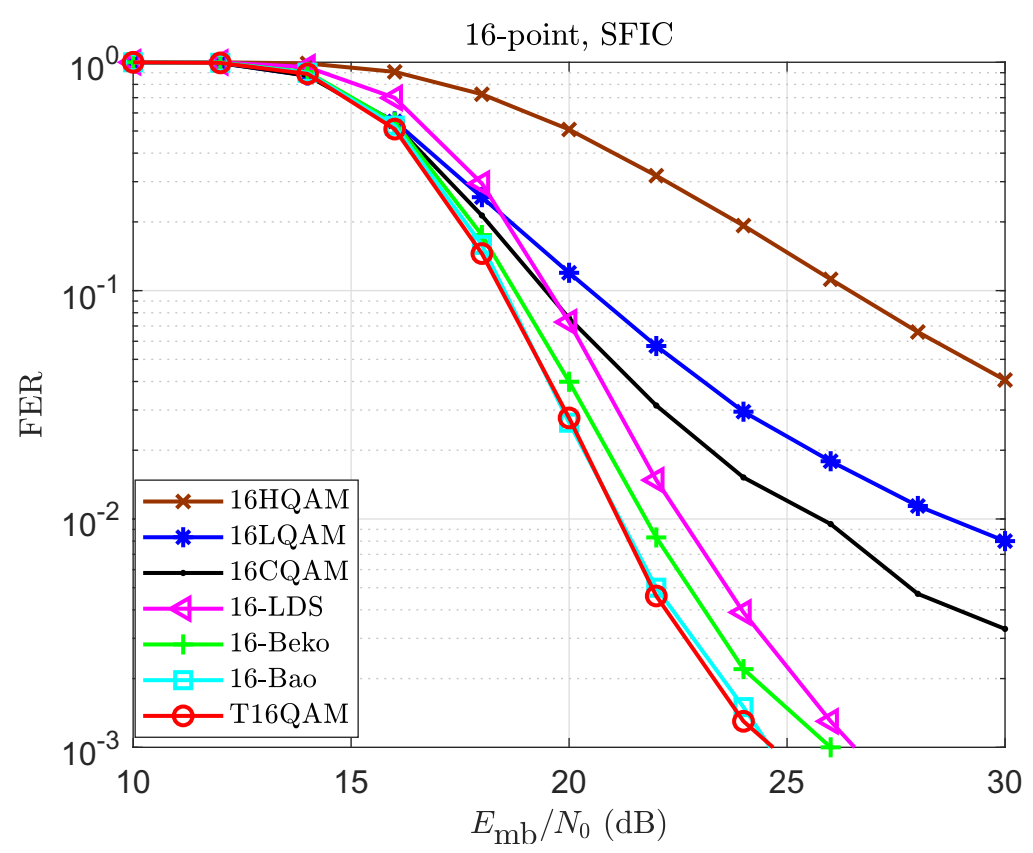

Figure 3.32: FER performance of $R=4 / 5$ turbo-coded SCMA systems with 16point constellations over SFIC (Case 6).

i.e., Fig. 3.23 and Fig. 3.24.

\subsubsection{Low-Rate Turbo-coded Scenarios}

In this section, we evaluate the FER performance of low-rate turbo-coded SCMA systems with 4-point and 16-point 2-dimensional complex constellations with respect to SNR in different scenarios. We define SNR as the same way as (3.16). We employ an LTE turbo code with a rate of $R=1 / 3$, a codeword length of $N_{c}=2028$, and 4 turbo iterations.

In Fig. 3.33-3.40, we compare the FER performance of turbo-coded SCMA systems with $R=1 / 3$ and different constellations over FFSC, FFIC, SFSC, and SFIC.

As mentioned earlier, depending on the behavior of the multiuser detector, the system performs differently at different SNRs. In fast fading and especially in the presence of low-rate (more powerful) code, due to the high amount of diversity introduced by the channel, a low FER can be achieved at lower SNRs. Fig. 3.33-3.36 


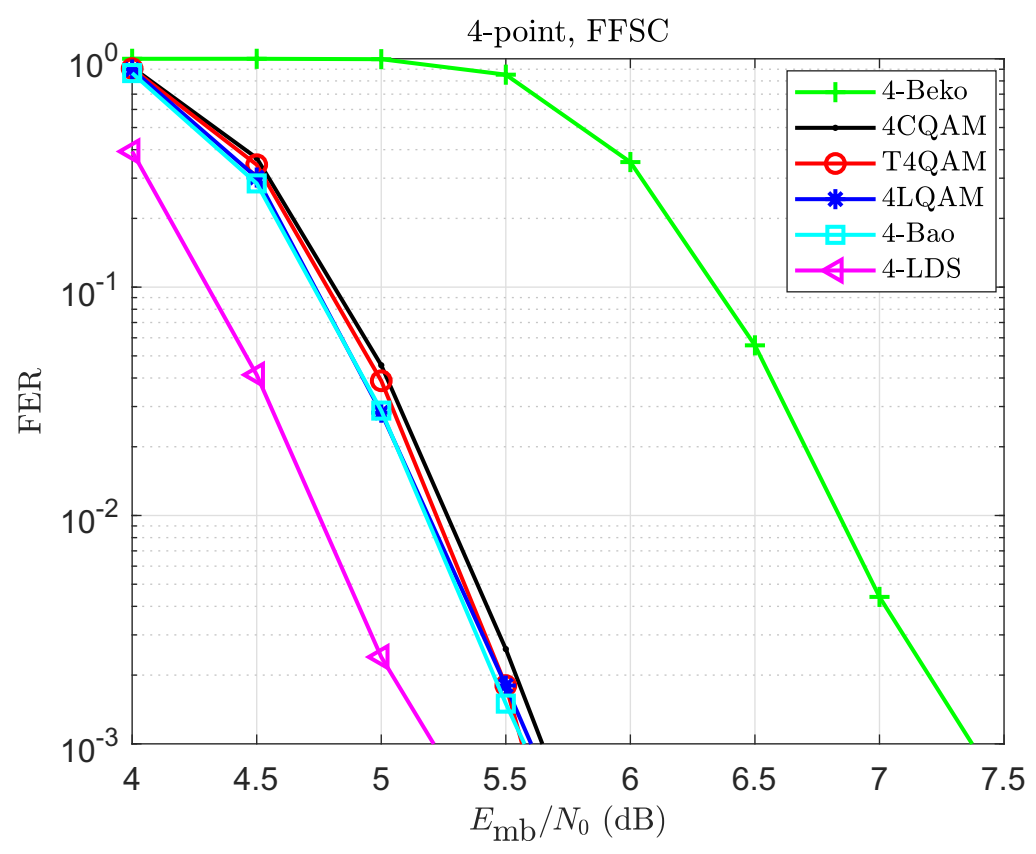

Figure 3.33: FER performance of $R=1 / 3$ turbo-coded SCMA systems with 4-point constellations over FFSC (Case 3).

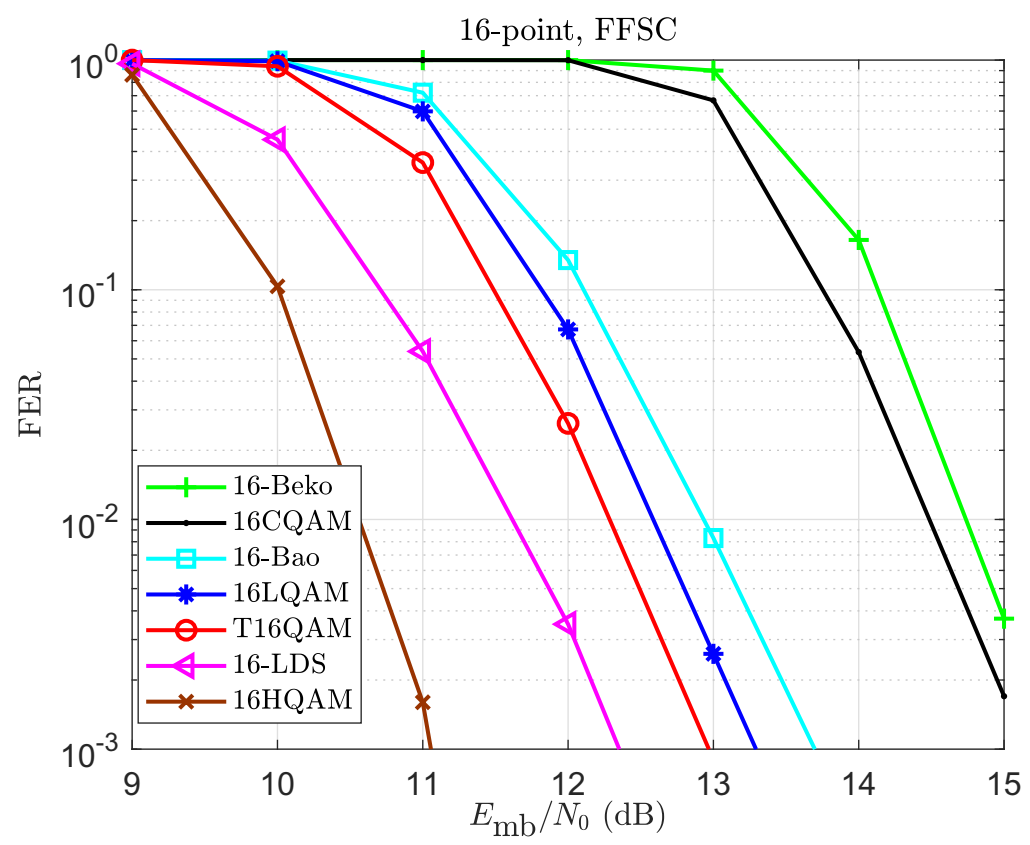

Figure 3.34: FER performance of $R=1 / 3$ turbo-coded SCMA systems with 16point constellations over FFSC (Case 3). 


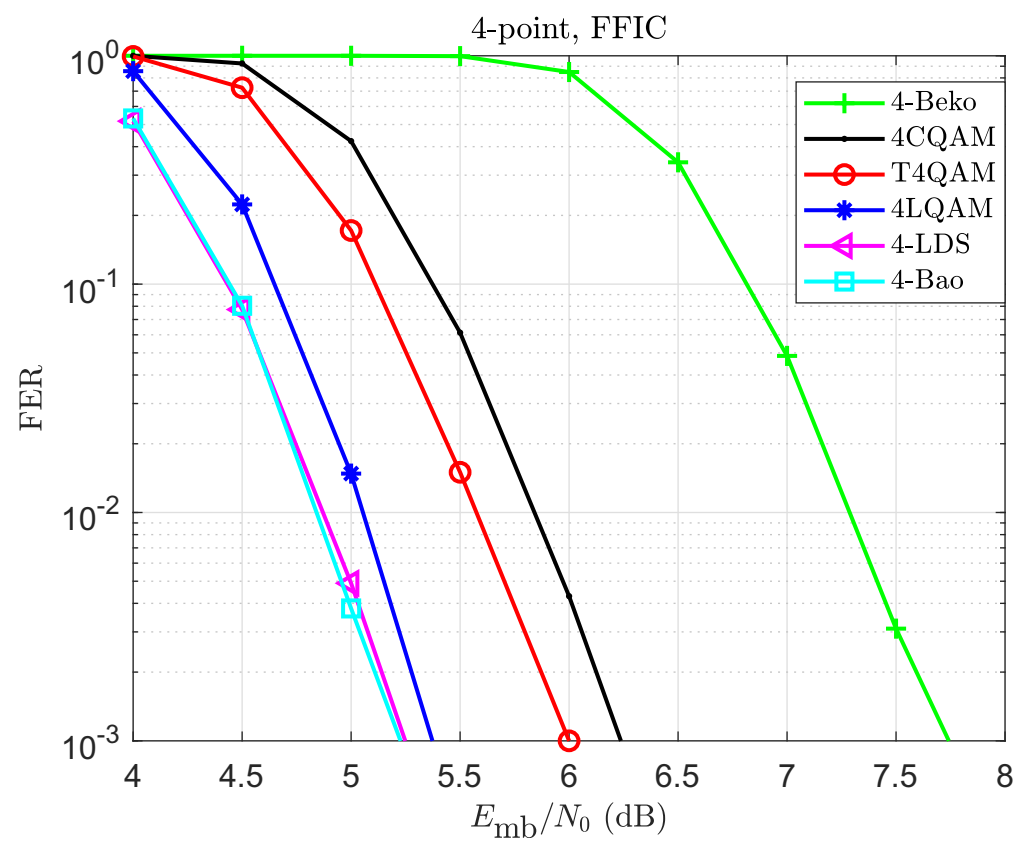

Figure 3.35: FER performance of $R=1 / 3$ turbo-coded SCMA systems with 4-point constellations over FFIC (Case 4 ).

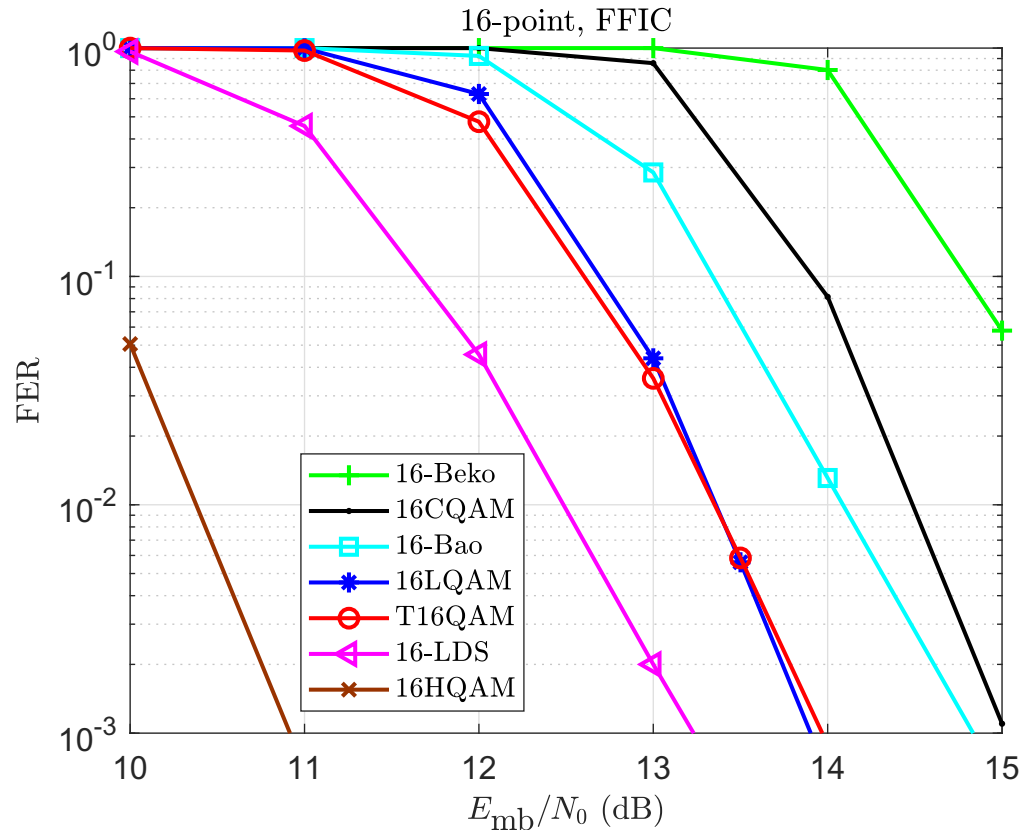

Figure 3.36: FER performance of $R=1 / 3$ turbo-coded SCMA systems with 16point constellations over FFIC (Case 4). 
evaluate the performance of $R=1 / 3$ systems at FFSC and FFIC. We see that the trends of different constellations are fairly consistent with the trends of BER performance of the uncoded systems at their corresponding low SNRs in Fig. 3.21-3.24. More specifically, 4-LDS outperforms the other 4-point constellations in FFSC and FFIC, while 16HQAM performs better than the other 16-point constellations in FFSC and FFIC.

In Fig. 3.37-3.38, we show the FER performance of the systems described above with $R=1 / 3$ over SFSC. We note that unlike the case of high-rate codes (Section 3.5.3), in the presence of a low-rate code, the effect of $N_{d}$ is less significant in this scenario. From Fig. 3.37 and referring to Table 3.2, we see that since all the Graylabeled constellations have the same $d_{E \text {,min }}$ and $\tau_{E}$, they perform similarly. On the other hand, 4-Beko lags behind the other constellations due to its bit-labeling. From Fig. 3.38 and referring to Table 3.3, we observe that among the Gray-labeled constellations, T16QAM, 16LQAM, 16HQAM, and 16-Bao, outperform the others. This is because they all have the same $d_{E \text {,min }}$ and $\tau_{E}$. Further, 16-LDS performs worse than the other Gray-labeled constellations due to its lower $d_{E \text {,min }}$. Also, 16-Beko and 16CQAM are behind the others due to their non-Gray bit-labeling.

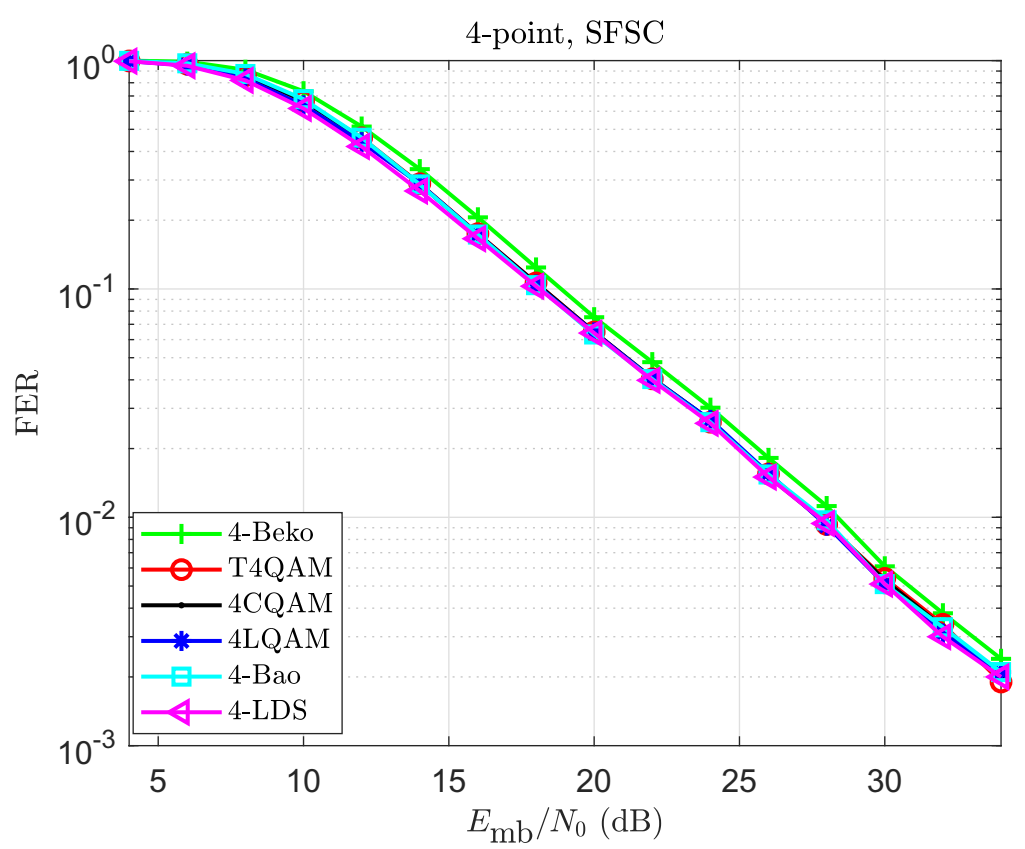

Figure 3.37: FER performance of $R=1 / 3$ turbo-coded SCMA systems with 4-point constellations over SFSC (Case 5). 


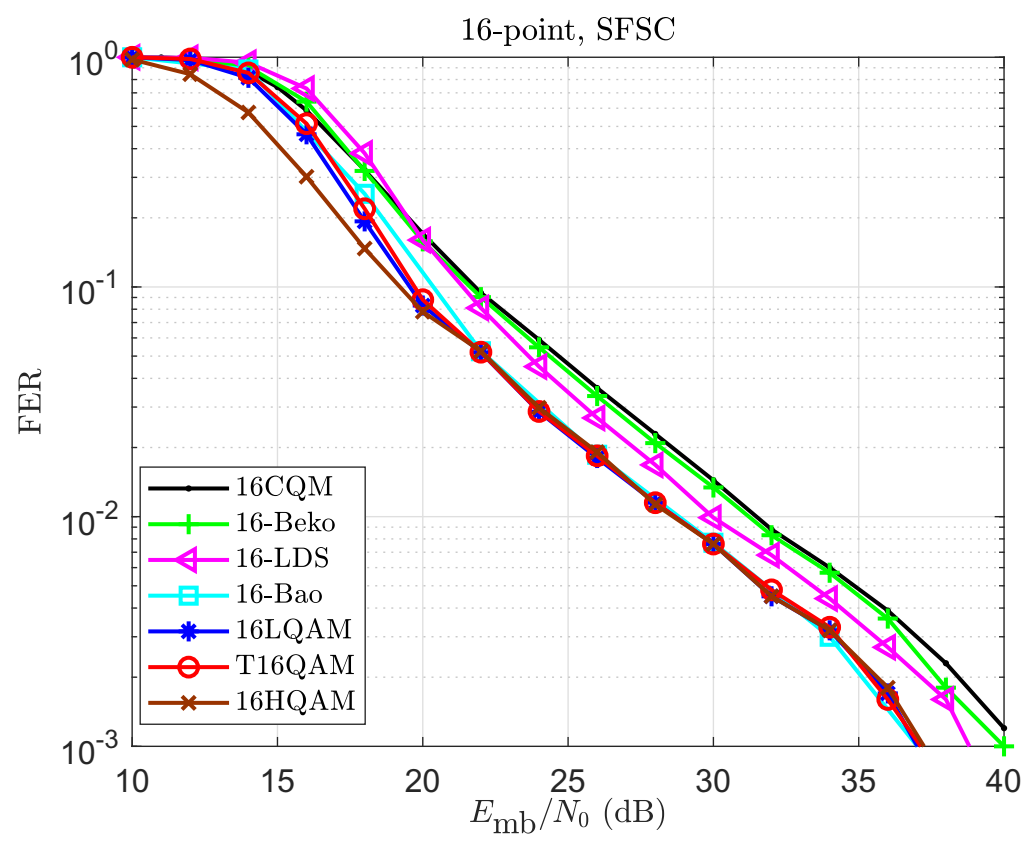

Figure 3.38: FER performance of $R=1 / 3$ turbo-coded SCMA systems with 16point constellations over SFSC (Case 5).

Fig. 3.39-3.40 present the FER performance of the systems mentioned above over SFIC. We see from Fig. 3.39 and Table 3.2 that 4-Bao and 4-LDS outperform the other constellations, as they both have similar KPI values. Nevertheless, from Fig. 3.40, we see that T16QAM outperforms the others. As pointed in [30], T16QAM is constructed similar to [71]. It was shown by Knopp and Humblet that constellations constructed the same way as $[70,71]$ are well suited for block fading channels with independent fading [100].

\subsubsection{Summary of Results}

In Table 3.4, we provide a summary of KPIs that should be considered in the design process of multidimensional constellations for uplink SCMA systems over different scenarios.

Based on the results provided in Section 3.5.2-3.5.4, we summarize the best 4point and 16-point constellation(s) for different scenarios in Table 3.5. Note that 


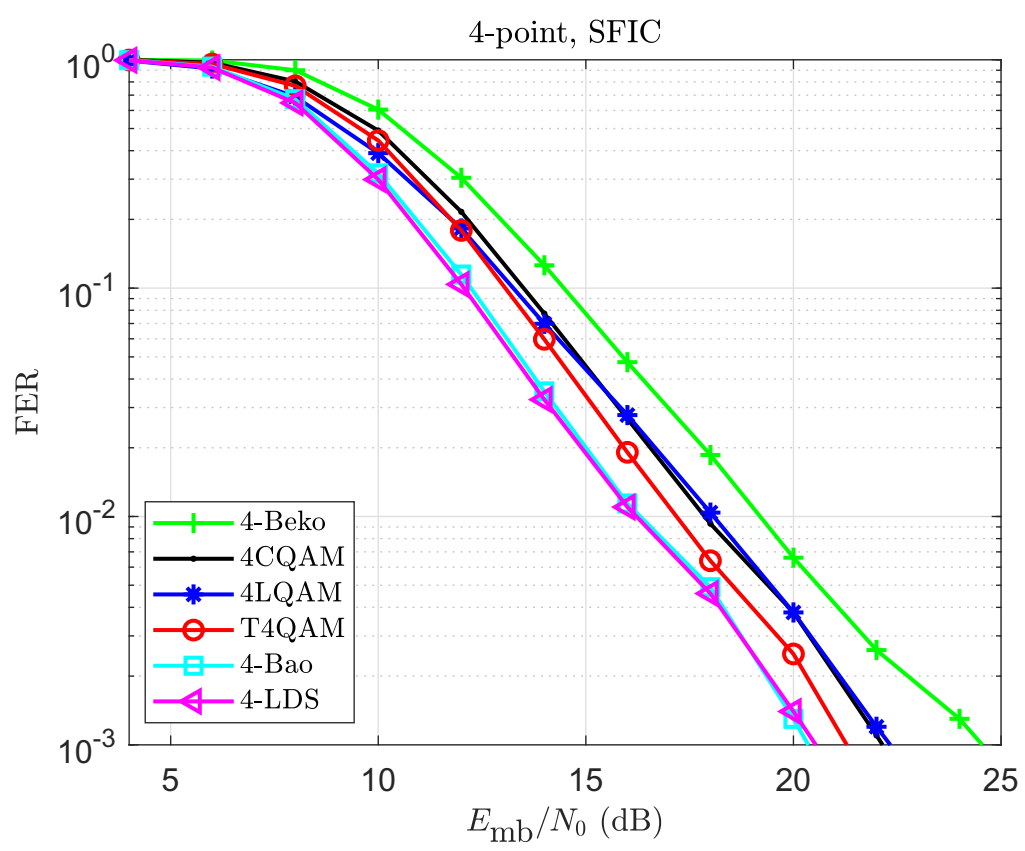

Figure 3.39: FER performance of $R=1 / 3$ turbo-coded SCMA systems with 4-point constellations over SFIC (Case 6).

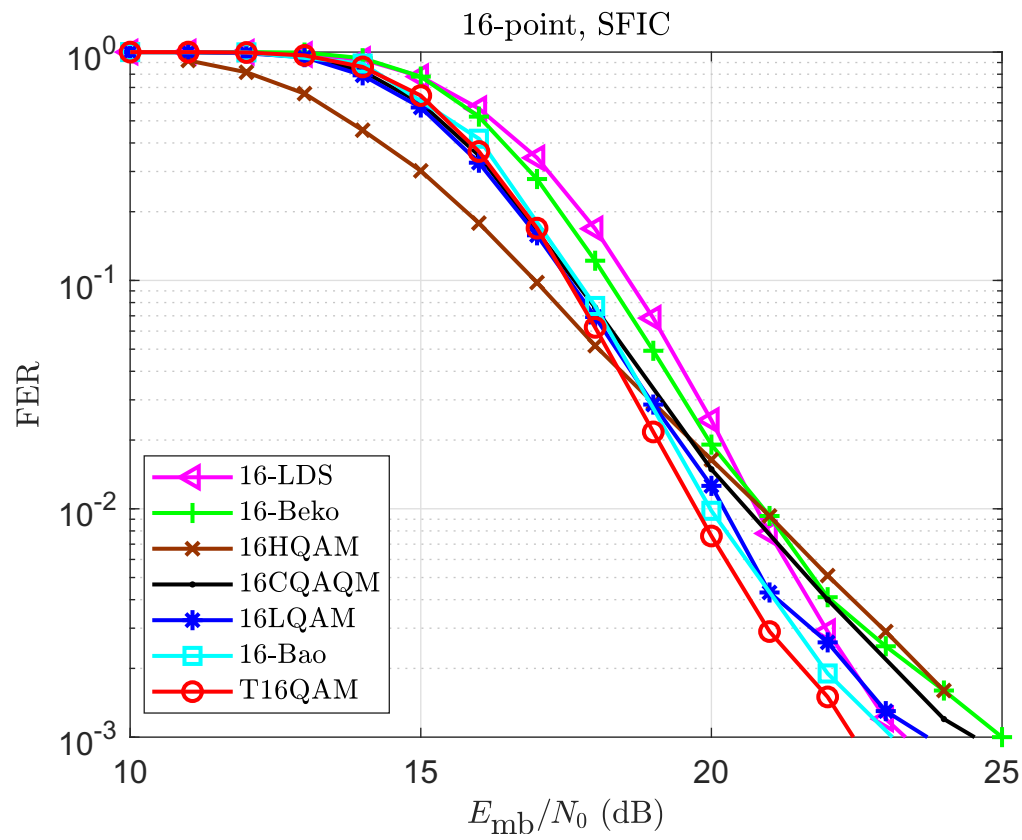

Figure 3.40: FER performance of $R=1 / 3$ turbo-coded SCMA systems with 16point constellations over SFIC (Case 6). 
Table 3.4: KPIs of multidimensional constellations for different channel scenarios

\begin{tabular}{ccccccc}
\hline & FSC & FIC & FFSC & FFIC & SFSC & SFIC \\
\hline$d_{E, \text { min }}^{2}$ & $\checkmark$ & & $\checkmark$ & & $\checkmark$ & \\
$\tau_{E}$ & $\checkmark$ & & $\checkmark$ & & $\checkmark$ & \\
$d_{P, \text { min }}^{2}$ & & $\checkmark$ & & $\checkmark$ & & $\checkmark$ \\
$\tau_{P}$ & & $\checkmark$ & & $\checkmark$ & & $\checkmark$ \\
$L$ & & $\checkmark$ & & $\checkmark$ & & $\checkmark$ \\
$N_{d}$ & $\checkmark$ & $\checkmark$ & $\checkmark$ & $\checkmark$ & $\checkmark$ & $\checkmark$ \\
Bit-labeling & $\checkmark$ & $\checkmark$ & $\checkmark$ & $\checkmark$ & $\checkmark$ & $\checkmark$ \\
\hline
\end{tabular}

Table 3.5: Best known multidimensional constellations for different channel scenarios

\begin{tabular}{ccc}
\hline Channel Scenario & Best 4-point Constellation(s) & Best 16-point Constellation(s) \\
\hline FSC (SER) & 4-Beko & 16-Beko, 16-Bao, T16QAM \\
FSC (BER) & 4 -LDS & 16-Bao, T16QAM \\
FIC (SER) & 4-Bao, 4-LDS & 16-Bao, T16QAM \\
FIC (BER) & 4 -Bao, 4-LDS & 16-Bao, T16QAM \\
High-rate FFSC (FER) & 4 -LDS & 16-Bao, T16QAM \\
High-rate FFIC (FER) & 4 -LDS & 16-Bao, T16QAM \\
High-rate SFSC (FER) & 4-Beko, 4-Bao, 4-LDS & 16-Bao, T16QAM \\
High-rate SFIC (FER) & 4 -Bao, 4-LDS & 16-Bao, T16QAM \\
Low-rate FFSC (FER) & 4 -LDS & 16HQAM \\
Low-rate FFIC (FER) & 4 -Bao, 4-LDS & 16HQAM \\
Low-rate SFSC (FER) & 4 -LDS, 4-Bao, 4LQAM, 4CQAM, T4QAM & 16HQAM, T16QAM, 16LQAM, 16-Bao \\
Low-rate SFIC (FER) & 4 -Bao, 4-LDS & T16QAM \\
\hline
\end{tabular}

the objective of the current work is not to determine what the best constellation is, rather to provide guidance of designing new efficient constellations for uplink SCMA systems. 


\subsection{Conclusion}

In this chapter, we provided a comparative study on some of the most important existing $M$-point $2 d_{v}$-dimensional constellations for SCMA systems under various channel conditions. Since SCMA has been proposed to support uplink machine-type communication services, we have only focused on uplink SCMA systems. We began our discussion with identifying the KPIs of constellations that are of significance for different channel scenarios. We then presented different existing constellations with their design criteria, and evaluated the performance of an important subset of those constellations for uncoded, high-rate and low-rate LTE turbo-coded SCMA systems under various channel scenarios. All turbo-coded comparisons were performed for BICM, with a concatenated detection and decoding scheme.

This chapter sheds some light in designing multidimensional constellations for SCMA systems over a variety of channel conditions. When designing an $M$-point $2 d_{v^{-}}$ dimensional constellation for uplink uncoded SCMA systems over FSC and high-rate turbo-coded systems over FFSC and SFSC, it is imperative to consider maximizing $N_{d}$ and $d_{E \text {,min }}$, and minimizing $\tau_{E}$. In systems with a low rate code over FFSC and SFSC, the effect of $N_{d}$ is of less concern. On the other hand, in the design process of an $M$-point $2 d_{v}$-dimensional constellations for uplink SCMA systems over FIC, FFIC, and SFIC, it is desired to maximize $N_{d}$, minimize $L$, maximize $d_{P, \min }$, and minimize $\tau_{P}$. Furthermore, the BER performance of the uncoded systems, and the performance of the coded systems are tied to their bit-labeling. The performance of the systems also highly depends on the behavior of the multiuser detector at different SNRs. 


\section{Chapter 4}

\section{Hypercube-based SNR-adaptive Multidimensional Constellation Design for Uplink SCMA Systems}

\subsection{Introduction}

As the performance of an $\mathrm{MdC}$ depends on the channel condition, it is of significance to use an MdC suited to a certain channel scenario. The KPIs that should be considered in designing MdCs over different channel conditions for uplink SCMA systems are provided in Chapter 3. Moreover, a comprehensive study of formerly proposed $\mathrm{MdCs}$ is presented, and the performance of an important subclass of those MdCs is evaluated under various scenarios for uncoded, high-rate, and low-rate LTE turbocoded [39] SCMA systems. All comparisons are made for BICM. The trends of most MdCs in the presence of 5G-compliant LDPC codes [40] are shown in Appendix D to be fairly consistent with their trends when the LTE turbo code is employed.

As mentioned in Section 3.3, the Gray-labelled MdCs that are constructed based on hypercubes perform well when used along with BICM as they obtain an equal error protection among their different bit levels. It is pointed out in Section 3.4 that the T16QAM (Fig. 3.7) and 16-Bao (Fig. 3.9) constellations, which outperform other constellations in most channel conditions, are both based on the rotation of hypercubes. Due to the attractive features of hypercubes, we construct MdCs that are based on a unitary rotation of a hypercube by a certain rotation angle. The objective of this chapter is to find an optimum rotation angle that results in a minimum FER in a coded uplink SCMA system employing BICM. We focus on 5G-compliant LDPC 
codes, but our findings are also applicable to other forward error correction (FEC) techniques, e.g., LTE turbo codes. We use Log-MPA (Appendix C), for MUD and do MUD and LDPC decoding separately in a concatenated way. To maintain focus, we also consider a quasi-static fading channel, SFIC (Case 6), where the channel coefficients for each user stays constant for the duration of the entire codeword, and each user experiences independent channel coefficients over its assigned RE.

It is known that the FER performance of a system depends on its SNR operating region [67]. Moreover, as MPA depends on the number of iterations (Appendix C), the FER performance varies with the MPA number of iterations. As such, obtaining a closed-form expression for FER as a function of the optimum rotation angle, SNR, and the MPA number of iterations for a coded SCMA system under a certain channel condition is difficult. At a given SNR and for a certain number of MPA iterations, one brute-force way to find the optimum rotation angle is to perform an exhaustive search over the entire range of the angles. Clearly, such a brute-force approach is burdensome and is not practical. Instead, using the special properties of a hypercube, we limit the search space of the rotation angle to reduce the search complexity substantially compared with the exhaustive search, and still result in an optimum rotation angle. Further, using the Levenberg-Marquardt algorithm for nonlinear least squares curvefitting [101], we propose an efficient algorithm that is able to find a close-to-optimal rotation angle with much lower complexity than the exhaustive search. Simulation results demonstrate the the resultant MdCs significantly outperform the existing ones, especially at low to mid SNR operating regions.

\subsection{SCMA MdC Design Problem}

As we mentioned in Chapter 3, in the uplink scenarios, random and different channel coefficients between different users are sufficient to ensure that the transmitted constellation symbols do not combine destructively. As such, we assume each $\tilde{\mathbf{x}}_{k, i}$, represented in Chapter 2 , is selected from a $d_{v}$-dimensional complex constellation, $\mathcal{X}=\left\{\mathbf{x}_{m} \mid m=1, \ldots, M\right\}$, with $M$ elements, where each element of $\mathcal{X}$ refers to a $d_{v^{-}}$

dimensional complex vector, $\mathbf{x}_{m}=\left(x_{1, m}, \ldots, x_{d_{v}, m}\right)^{\mathrm{T}}$. In this chapter, we propose a method to design $\mathcal{X}$ that outperforms the state of the art MdC in coded uplink SCMA systems over SFIC (Case 6). 


\subsubsection{Rotation-based MdCs}

As we discussed in Section 3.3, the Gray-labelled MdCs that are constructed based on hypercubes perform well when used along with BICM as they obtain an equal error protection among their different bit levels. We construct the $2 d_{v}$-dimensional real mother constellation, $\mathcal{X}(\theta)$, based on a $2 d_{v}$-by- $2 d_{v}$ unitary rotation of a hypercube by the angle of $\theta$. Let $\mathcal{X}^{+}=\left\{\mathbf{x}_{m}^{+} \mid m=1, \ldots, M\right\}$ denote a constellation wherein each of its elements is a corner of a $2 d_{v}$-dimensional hypercube. That is, $\mathbf{x}_{m}^{+}=\left(x_{m, 1}^{+}, \ldots, x_{m, 2 d_{v}}^{+}\right)^{\mathrm{T}}$, $x_{m, q}^{+} \in\{ \pm 1\}, q \in\left\{1, \ldots, 2 d_{v}\right\}$. Then,

$$
\mathcal{X}(\theta)=\left\{\mathbf{R}(\theta) \mathbf{x}_{m}^{+} \mid m=1, \ldots, M\right\},
$$

where $\mathbf{R}(\theta)$ is a function of a rotation angle, $\theta$, and represents a $2 d_{v}$-by- $2 d_{v}$ unitary rotation. By changing $\theta$ a different rotation matrix and, as a result, a different $\mathcal{X}(\theta)$ in (4.1) is attained. The unitary rotation $\mathbf{R}(\theta)$ is built based on Givens rotations, which are given by [102]

$$
\mathbf{G}_{u, v}(\theta)=\left[\begin{array}{ccccc}
\mathbf{I} & \vdots & \vdots & \vdots & \vdots \\
\ldots & c & \ldots & -s & \ldots \\
\ldots & s & \vdots & c & \vdots \\
\vdots & \vdots & \vdots & \vdots & \mathbf{I}
\end{array}\right],
$$

where $c=\cos \theta, s=\sin \theta$, and $\theta$ is the rotation angle along the $u$ th row and $v$ th column of $\mathbf{G}_{u, v}$.

Since an important application of SCMA systems is to fulfill the massive connectivity necessities in IoT applications, e.g., [80], and not to enhance the throughput of each user, we focus on $M=16$. Moreover, as a sparse user-to-RE spreading matrix, $\mathbf{S}_{k}$, is an essential requirement in an SCMA system, we focus on $d_{v}=2 .{ }^{1}$ For $d_{v}=2$ and $M=16$, the Cartesian product of two 4-QAM constellation results in the points lying on the 16 corners of a 4-dimensional hypercube. As in Section 3.4, we refer to the 16-point 4-dimensional hypercube-based QAM as 16HQAM, where the projections of 16HQAM onto $\mathrm{RE}_{1}$ and $\mathrm{RE}_{2}$ is shown with brown crosses in Fig. 4.1.

\footnotetext{
${ }^{1}$ Note that as mentioned in Chapter 3 , such a scenario has been the most commonly studied scenario so far, e.g., [1,30,51-53,55-64,103-105].
} 
For $d_{v}=2$, we consider the following Givens rotations:

$$
\begin{aligned}
\mathbf{G}_{1,4}(\theta) & =\left[\begin{array}{cccc}
c & 0 & 0 & -s \\
0 & 1 & 0 & 0 \\
0 & 0 & 1 & 0 \\
s & 0 & 0 & c
\end{array}\right] . \\
\mathbf{G}_{2,3}(\theta) & =\left[\begin{array}{llll}
1 & 0 & 0 & 0 \\
0 & c & -s & 0 \\
0 & s & c & 0 \\
0 & 0 & 0 & 1
\end{array}\right] .
\end{aligned}
$$

Then,

$$
\begin{aligned}
\mathbf{R}(\theta) & =\mathbf{G}_{1,4}(\theta) \\
& =\left[\begin{array}{cccc}
c & 0 & 0 & -s \\
0 & c & -s & 0 \\
0 & s & c & 0 \\
s & 0 & 0 & c
\end{array}\right] .
\end{aligned}
$$

Substituting (4.5) in (4.1),

$$
\begin{aligned}
& x_{m, 1}(\theta)=x_{m, 1}^{+} c-x_{m, 4}^{+} s, \\
& x_{m, 2}(\theta)=x_{m, 2}^{+} c-x_{m, 3}^{+} s, \\
& x_{m, 3}(\theta)=x_{m, 2}^{+} s+x_{m, 3}^{+} c, \\
& x_{m, 4}(\theta)=x_{m, 1}^{+} s+x_{m, 4}^{+} c .
\end{aligned}
$$

Proposition 1. If $\mathcal{X}^{+}$is Gray-labelled, $\mathcal{X}(\theta)$ is also Gray-labelled. 


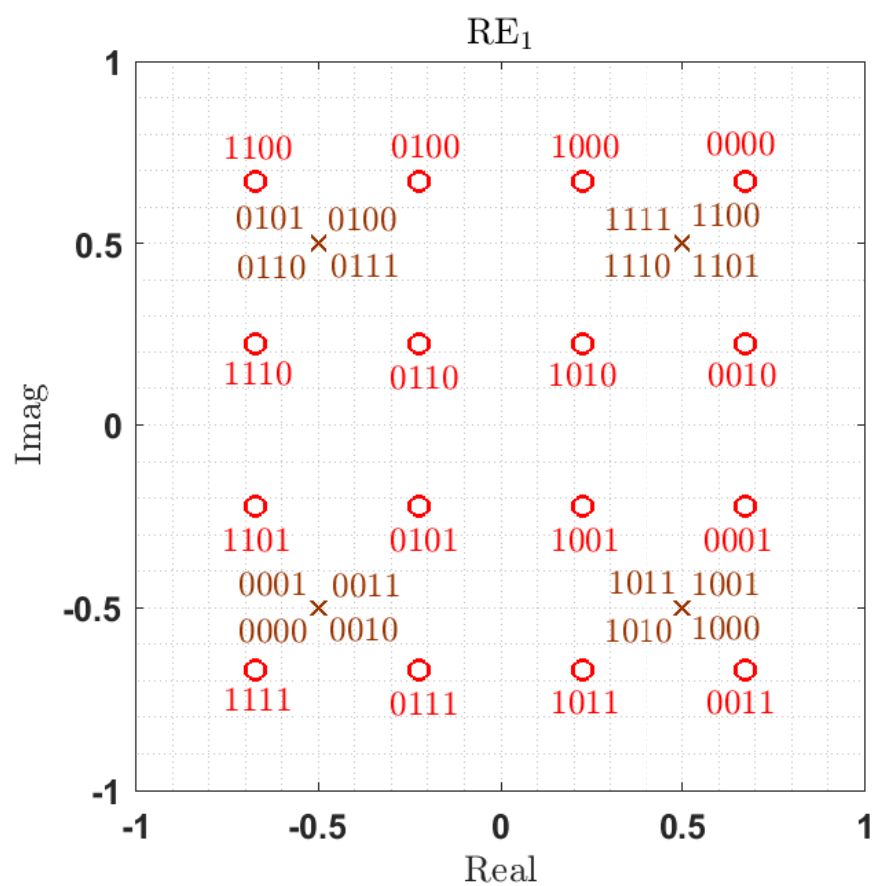

(a)

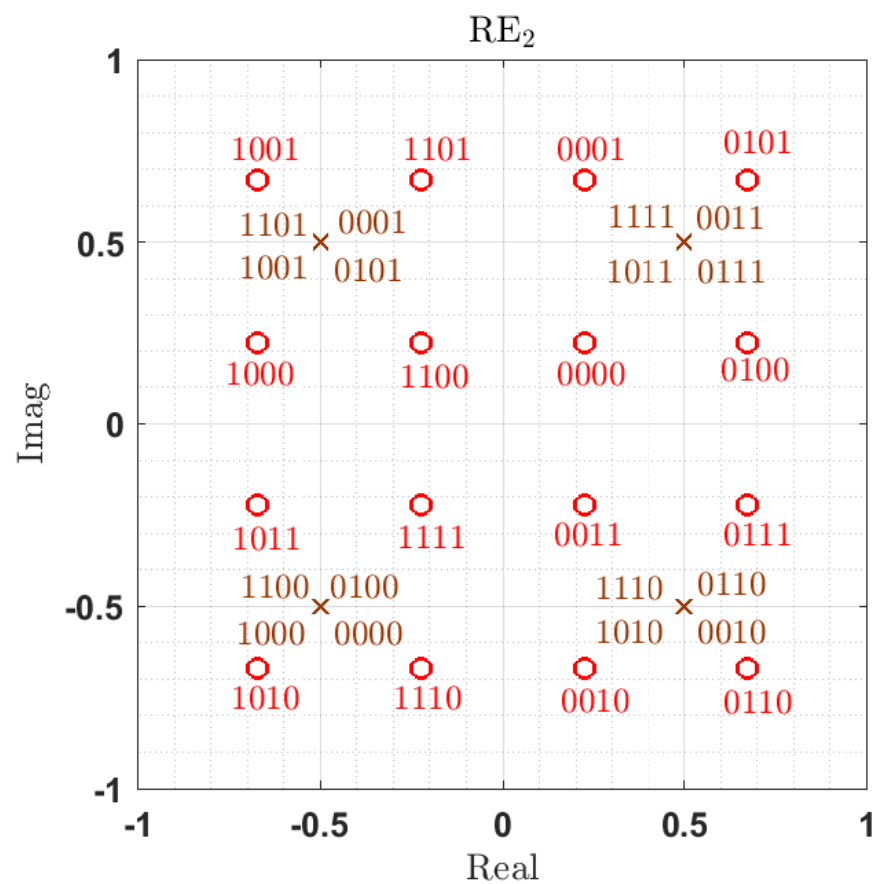

(b)

Figure 4.1: The projections of 16HQAM (brown crosses) and T16QAM (red circles) onto $\mathrm{RE}_{1}$ and $\mathrm{RE}_{2}$. 
Proof. Since the bit labelling is determined based on the Euclidean distance profile between constellation points [67] (e.g., the bit labels for adjacent points must differ in only one bit position for Gray labelling), it is sufficient to show that $\mathcal{X}(\theta)$ and $\mathcal{X}^{+}$ have identical Euclidean distance profiles.

Let $\mathbf{x}_{m}^{+}$and $\mathbf{x}_{m^{\prime}}^{+}, 1 \leq m<m^{\prime} \leq M$, represent any two constellation points in $\mathcal{X}^{+}$, with the squared Euclidean distance of $d_{E_{X^{+}}}^{m m^{\prime}}$, that for $d_{v}=2$ can be expressed as

$$
\begin{aligned}
d_{E_{X^{+}}}^{m m^{\prime}}= & \sum_{q=1}^{2 d_{v}}\left(x_{m, q}^{+}-x_{m^{\prime}, q}^{+}\right)^{2} \\
= & \left(x_{m, 1}^{+}-x_{m^{\prime}, 1}^{+}\right)^{2}+\left(x_{m, 2}^{+}-x_{m^{\prime}, 2}^{+}\right)^{2} \\
& +\left(x_{m, 3}^{+}-x_{m^{\prime}, 3}^{+}\right)^{2}+\left(x_{m, 4}^{+}-x_{m^{\prime}, 4}^{+}\right)^{2} .
\end{aligned}
$$

Let $\mathbf{x}_{m}=\mathbf{R}(\theta) \mathbf{x}_{m}^{+}$and $\mathbf{x}_{m^{\prime}}=\mathbf{R}(\theta) \mathbf{x}_{m^{\prime}}^{+}$, be the rotated points in $\mathcal{X}(\theta)$ corresponding to $\mathbf{x}_{m}^{+}$and $\mathbf{x}_{m^{\prime}}^{+}$, respectively. Their squared Euclidean distance, $d_{E_{X}}^{m m^{\prime}}$, from (4.6) and using the relationship, $\cos ^{2} \theta+\sin ^{2} \theta=1$, is

$$
\begin{aligned}
d_{E_{X}}^{m m^{\prime}}= & \sum_{q=1}^{2 d_{v}}\left(x_{m, q}-x_{m^{\prime}, q}\right)^{2} \\
= & \left(x_{m, 1}^{+}-x_{m^{\prime}, 1}^{+}\right)^{2} c^{2}+\left(x_{m, 4}^{+}-x_{m^{\prime}, 4}^{+}\right)^{2} s^{2}-2\left(x_{m, 1}^{+}-x_{m^{\prime}, 1}^{+}\right)\left(x_{m, 4}^{+}-x_{m^{\prime}, 4}^{+}\right) s c \\
& +\left(x_{m, 2}^{+}-x_{m^{\prime}, 2}^{+}\right)^{2} c^{2}+\left(x_{m, 3}^{+}-x_{m^{\prime}, 3}^{+}\right)^{2} s^{2}-2\left(x_{m, 2}^{+}-x_{m^{\prime}, 2}^{+}\right)\left(x_{m, 3}^{+}-x_{m^{\prime}, 3}^{+}\right) s c \\
& +\left(x_{m, 2}^{+}-x_{m^{\prime}, 2}^{+}\right)^{2} s^{2}+\left(x_{m, 3}^{+}-x_{m^{\prime}, 3}^{+}\right)^{2} c^{2}+2\left(x_{m, 2}^{+}-x_{m^{\prime}, 2}^{+}\right)\left(x_{m, 3}^{+}-x_{m^{\prime}, 3}^{+}\right) s c \\
& +\left(x_{m, 1}^{+}-x_{m^{\prime}, 1}^{+}\right)^{2} s^{2}+\left(x_{m, 4}^{+}-x_{m^{\prime}, 4}^{+}\right)^{2} c^{2}+2\left(x_{m, 1}^{+}-x_{m^{\prime}, 1}^{+}\right)\left(x_{m, 4}^{+}-x_{m^{\prime}, 4}^{+}\right) s c \\
= & \left(x_{m, 1}^{+}-x_{m^{\prime}, 1}^{+}\right)^{2}\left(c^{2}+s^{2}\right)+\left(x_{m, 4}^{+}-x_{m^{\prime}, 4}^{+}\right)^{2}\left(c^{2}+s^{2}\right) \\
& +\left(x_{m, 2}^{+}-x_{m^{\prime}, 2}^{+}\right)^{2}\left(c^{2}+s^{2}\right)+\left(x_{m, 3}^{+}-x_{m^{\prime}, 3}^{+}\right)^{2}\left(c^{2}+s^{2}\right) \\
= & d_{E_{X^{+}}}^{m m^{\prime}} .
\end{aligned}
$$

As such, $\mathcal{X}$ and $\mathcal{X}^{+}$have identical Euclidean distance profiles. As a result if $\mathbf{x}_{m}^{+}$and $\mathbf{x}_{m^{\prime}}^{+}$are two adjacent points in $\mathcal{X}^{+}$and they differ in one bit position, their rotated versions, $\mathbf{x}_{m}$ and $\mathbf{x}_{m^{\prime}}$ are two adjacent points in $\mathcal{X}$ and differ in one bit position. In other words, if $\mathcal{X}^{+}$is Gray-labelled, $\mathcal{X}(\theta)$ is also Gray-labelled.

\subsubsection{Problem Formulation}

The objective of this work is to find an optimal angle, $\theta_{\mathrm{opt}}$, corresponding to an $\mathrm{MdC}$ 
that minimizes the FER performance of an LDPC-coded uplink SCMA system at a certain SNR and for the channel model under study. On the other hand, obtaining a closed-form expression for the FER of an LDPC-coded SCMA system is difficult, and depends on the detection technique. For a single-user system with $d_{v}=2$ that employs the optimal MAP detection, the optimal rotation angle, $\theta_{\text {opt }}$, for all SNRs is found numerically through exhaustive search to be equal to $\arctan (3)-\pi / 4$, and we refer to this angle as $\theta_{\mathrm{mx}}$. Rotating $\mathcal{X}^{+}$by $\theta_{\mathrm{mx}}$ results in the widely-used T16QAM constellation, which is described in Section 3.4, and is shown with red circles in Fig. 4.1. Also, for an uncoded SCMA system that employs the optimal maximum likelihood (ML) detection, the BER for all SNR regions reduces by increasing $\theta$ from 0 until it hits $\theta_{\mathrm{mx}}$, where the BER reaches its minimum; by increasing $\theta>\theta_{\mathrm{mx}}$, the BER starts increasing again. From Section 3.5, since the BER performance of an uncoded system at a certain SNR provides an indication of the FER performance at its corresponding SNR when coding is used, we can conclude that by employing the MAP detection in (2.5) to detect each coded SCMA symbol, the optimal angle also occurs at $\theta_{\mathrm{mx}}$.

As mentioned in Chapter 2, the optimal MAP detection (2.5) is prohibitively complex, and the near-optimal MPA is commonly employed to detect each SCMA symbol. The MPA detection is described in detail in Appendix C, and is a function of the number of iterations. Particularly, the MPA performance improves by increasing the number of iterations, and with a sufficient number of iterations is expected to approach the optimal MAP detection (e.g., [28]). Let $N_{\text {it }}$ denote the number of MPA iterations; it is shown in Section 5.3 that even with $N_{\text {it }}=12$ when the T16QAM constellation is used, the MPA performance is still off from the optimal detection technique, especially at low SNRs. As we will discuss later in this section, by using MPA for detecting each SCMA symbol, $\theta_{\text {opt }}$ does not always occur at $\theta_{\mathrm{mx}}$, and its value depends on SNR and $N_{\text {it }}$. Finding a closed-form expression for the FER LDPCcoded SCMA system with the MPA detection for a given $N_{\text {it }}$ is complicated - if not impossible. We can numerically search for the value of $\theta_{\text {opt }}$ in the range $[0,2 \pi]$ that minimizes the FER. The following propositions show that it is sufficient to limit the range of the search for $\theta_{\text {opt }}$ to $[0, \pi / 4]$ instead of $[0,2 \pi]$, which reduces the complexity of the exhaustive search by $87.5 \%$. We denote the FER of the system with the MdC generated with the rotation angle of $\theta$ by $\operatorname{FER}(\theta)$.

Proposition 2. At a given $S N R$ and for a certain $N_{i t}, F E R(\theta)$ is a periodic function 
with a period of $\theta=\pi / 2$. In other words, $F E R(\theta)=F E R(\theta+\pi / 2)$.

Proof. We only need to show that $\mathcal{X}(\theta)=\left\{\mathbf{R}(\theta) \mathbf{x}_{m}^{+}\right\}$and $\mathcal{X}(\theta+\pi / 2)=\left\{\mathbf{R}(\theta+\pi / 2) \mathbf{x}_{m}^{+}\right\}$ result in the same error performance. Using the trigonometric identities, $\cos (\theta+\pi / 2)=$ $-\sin \theta$ and $\sin (\theta+\pi / 2)=\cos \theta$ with (4.5), we obtain

$$
\mathbf{R}(\theta+\pi / 2)=\left[\begin{array}{cccc}
-s & 0 & 0 & -c \\
0 & -s & -c & 0 \\
0 & c & -s & 0 \\
c & 0 & 0 & -s
\end{array}\right]
$$

If we define

$$
\mathbf{W}_{1}=\left[\begin{array}{cccc}
0 & 0 & 0 & -1 \\
0 & 0 & -1 & 0 \\
0 & 1 & 0 & 0 \\
1 & 0 & 0 & 0
\end{array}\right]
$$

then it is easy to confirm that $\mathbf{R}(\theta+\pi / 2)=\mathbf{R}(\theta) \mathbf{W}_{1}$, so $\mathcal{X}(\theta+\pi / 2)=\left\{\mathbf{R}(\theta+\pi / 2) \mathbf{x}_{m}^{+}\right\}=$ $\left\{\mathbf{R}(\theta) \mathbf{W}_{1} \mathbf{x}_{m}^{+}\right\}=\left\{\mathbf{R}(\theta) \mathbf{d}_{m}\right\}$, where $\mathbf{d}_{m}=\mathbf{W}_{1} \mathbf{x}_{m}^{+}$. That is, symbols in $\mathcal{X}(\theta+\pi / 2)$ can be generated by transforming the points in the hypercube by multiplying them by $\mathbf{W}_{1}$, and then rotating them using $\mathbf{R}(\theta)$. The transformation by $\mathbf{W}_{1}$ is trivial and can easily be implemented digitally by a) reversing the order of the code bits, and b) inverting every second pair of code bits. Because LDPC codes are linear, and because all bit positions in the constellations are equally reliable ${ }^{2}$ neither reordering nor inverting code bits will have any impact on the FER of the system. As such, although $\mathcal{X}(\theta)$ and $\mathcal{X}(\theta+\pi / 2)$ are different (more precisely, they contain the same points but with different labelling) they are equivalent and provide the same FER when used with linear codes in SCMA systems.

Proposition 3. At a given $S N R$ and for a certain $N_{i t}$, FER $(\theta)$ reflects at $\theta=\pi / 4$. In other words, $\operatorname{FER}(\pi / 4+\theta)=\operatorname{FER}(\pi / 4-\theta)$.

\footnotetext{
${ }^{2}$ This is a property of hypercube-based constellations and can be confirmed by comparing the code bit LLR densities at the receiver.
} 
Proof. We only need to show that $\mathcal{X}(\pi / 4+\theta)$ and $\mathcal{X}(\pi / 4-\theta)$ are equivalent and therefore result in the same error performance. From (4.5), we obtain

$$
\begin{aligned}
& \mathbf{R}(\pi / 4+\theta)=\left[\begin{array}{cccc}
A & 0 & 0 & -B \\
0 & A & -B & 0 \\
0 & B & A & 0 \\
B & 0 & 0 & A
\end{array}\right], \\
& \mathbf{R}(\pi / 4-\theta)=\left[\begin{array}{cccc}
B & 0 & 0 & -A \\
0 & B & -A & 0 \\
0 & A & B & 0 \\
A & 0 & 0 & B
\end{array}\right],
\end{aligned}
$$

where $A=\frac{\sqrt{2}}{2}[\cos \theta-\sin \theta]$ and $B=\frac{\sqrt{2}}{2}[\cos \theta+\sin \theta]$.

Observing that for

$$
\mathbf{W}_{\mathrm{L}}=\left[\begin{array}{cccc}
1 & 0 & 0 & 0 \\
0 & 1 & 0 & 0 \\
0 & 0 & -1 & 0 \\
0 & 0 & 0 & -1
\end{array}\right], \mathbf{W}_{\mathrm{R}}=\left[\begin{array}{cccc}
0 & 0 & 0 & -1 \\
0 & 0 & -1 & 0 \\
0 & -1 & 0 & 0 \\
-1 & 0 & 0 & 0
\end{array}\right] \text {. }
$$

We have $\mathbf{R}(\pi / 4+\theta)=\mathbf{W}_{\mathrm{L}} \mathbf{R}(\pi / 4-\theta) \mathbf{W}_{\mathrm{R}}$, so the only differences between generating symbols in $\mathcal{X}(\pi / 4-\theta)$ and $\mathcal{X}(\pi / 4+\theta)$ is that for $\mathcal{X}(\pi / 4+\theta)$ the points in the hypercube are transformed by $\mathbf{W}_{\mathrm{R}}$ prior to applying the unitary rotation by $\pi / 4-\theta$, and transformed again afterwards by $\mathbf{W}_{\mathrm{L}}$. As noted earlier, the first transformation has no effect on the system performance since it is equivalent to reordering and inverting the code bits. The second transformation also has no effect, since it is equivalent to rotating by 180 degrees the complex symbol component transmitted over $\mathrm{RE}_{2}$ for each user. From Chapter 3 , the effect of such a rotation is neutralized by the random channel rotations of each symbol transmitted in each channel in 


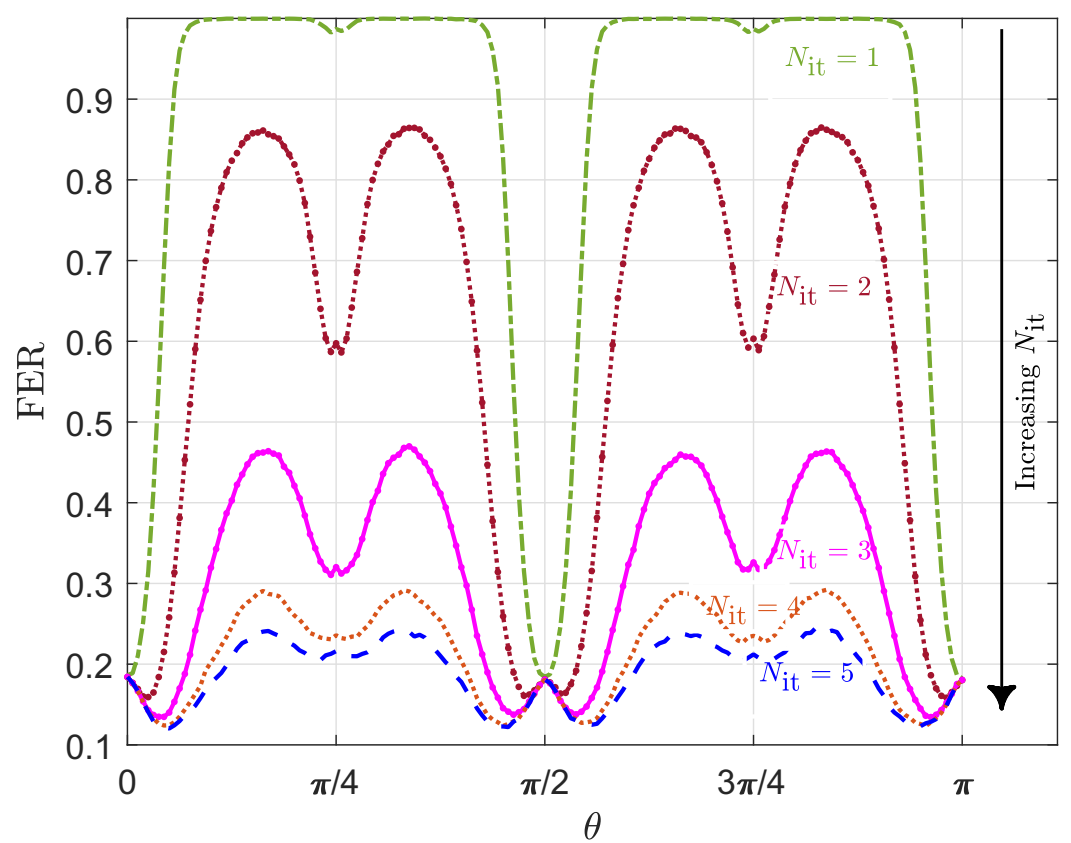

Figure 4.2: The effect of changing $\theta$ on FER at $E_{\mathrm{mb}} / N_{0}=17 \mathrm{~dB}$ for different $N_{\mathrm{it}} \mathrm{s}$.

uplink scenarios. Thus, $\mathcal{X}(\pi / 4+\theta)$ and $\mathcal{X}(\pi / 4-\theta)$ are equivalent constellations so $\operatorname{FER}(\pi / 4+\theta)=\operatorname{FER}(\pi / 4-\theta)$.

For illustrative purposes, in Fig. 4.2, using Monte Carlo simulation we show the FER performance of an SCMA system with $K=6, N=4, d_{v}=2$; each user employs a 5G-compliant LDPC code [40] with a rate of $R=1 / 3$ and $K_{c}=40$, under the quasi-static fading channel (Case 6 ), and for different $N_{\text {it }}$ when $\theta$ varies from 0 to $\pi$. The results in Fig. 4.2 are for $E_{\mathrm{mb}} / N_{0}=17 \mathrm{~dB}$, where $E_{\mathrm{mb}}$ denote the average energy per message bit as in Section 3.5. We see from Fig. 4.2 that for a given SNR and $N_{\text {it }}$, $\operatorname{FER}(\theta)$ is periodic with a period of $\theta=\pi / 2$ and reflects at $\theta=\pi / 4$. As expected, we also observe that increasing $N_{\text {it }}$ reduces the FER. We also observe from Fig. 4.2 that the value of $\theta$ that results in a minimum FER is different for different $N_{\mathrm{it}}$.

Since an important application of SCMA systems is to fulfill the massive connectivity requirements in IoT applications [80] in which delay can be an issue [106], we consider $N_{\text {it }} \leqslant 5$. Also, as with the MAP detection, $\theta_{\text {opt }}$ is equal to $\theta_{\mathrm{mx}}$, we expect that with the MPA detection at sufficiently high SNR regions and with sufficiently high $N_{\mathrm{it}}, \theta_{\mathrm{opt}}$ approaches $\theta_{\mathrm{mx}}$. Moreover, as shown in Fig. 4.1, $\theta_{\mathrm{mx}}$ results in a regular 
constellation, in which its projections over the two REs are essentially the 16QAM constellation with different labeling over each RE. As such, without loss of generality, we limit our search to the interval $\left[0, \theta_{\mathrm{mx}}\right]$, which would further reduces the complexity of the exhaustive search.

In Fig. 4.3-Fig. 4.7, we depict the FER performance of a 5G-compliant LDPC coded SCMA system with the same setting as in Fig. $4.2\left(K=6, N=4, d_{v}=2\right.$, $R=1 / 3, K_{c}=40$ ), with respect to SNR, for various values of $\theta$ that are expressed relative to $\theta_{\mathrm{mx}}$ and for different $N_{\mathrm{it}}$. As we can see in Fig. 4.3-Fig. 4.7, different values of $\theta$ result in different performance depending on the SNR and $N_{\mathrm{it}}$. For instance, to achieve a target FER of $10^{-2}$, depending on the system requirements, if delay is a key issue but not SNR, $\theta=0$ with $N_{\text {it }}=1$ is the best candidate; we refer to such a system as a $S N R$-tolerant system. On the other hand, if due to system requirements, SNR is limited but the system can tolerate some delay, $\theta=0.5 \theta_{\mathrm{mx}}$ with $N_{\mathrm{it}}=5$ is a better choice; we refer to such a system as a delay-tolerant system.

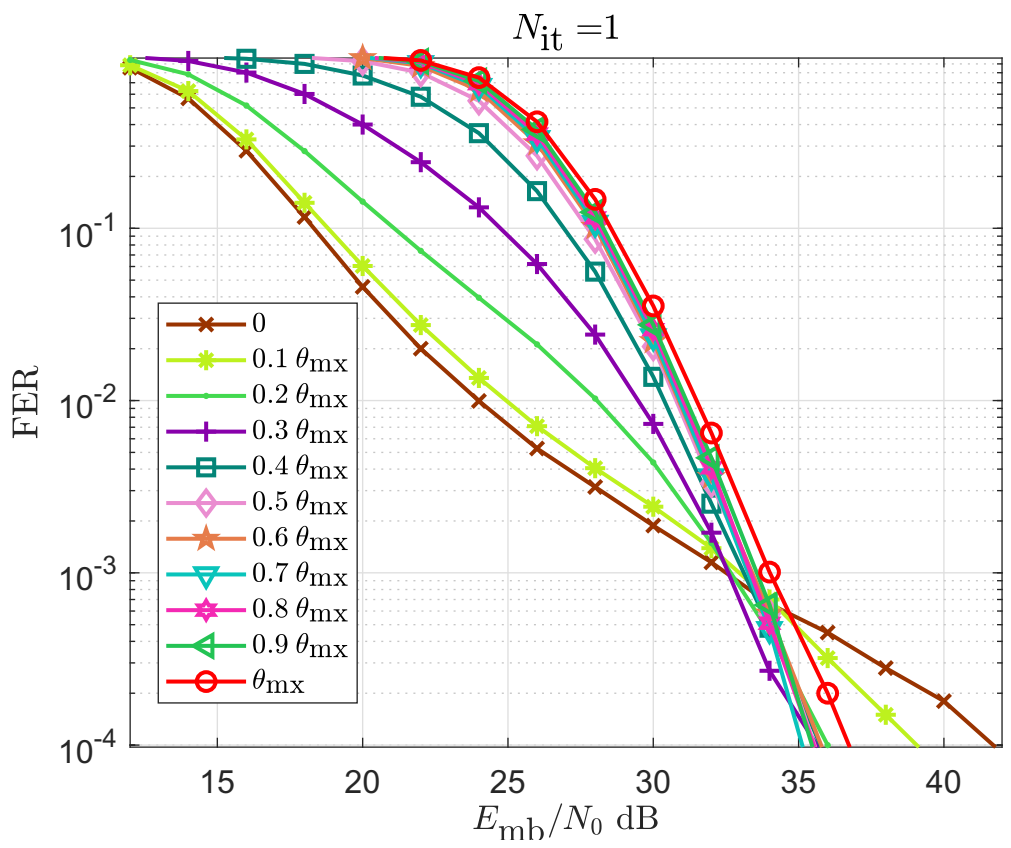

Figure 4.3: The effect of changing $\theta$ on the FER performance of an LDPC-coded SCMA systems with $N_{\text {it }}=1$. 


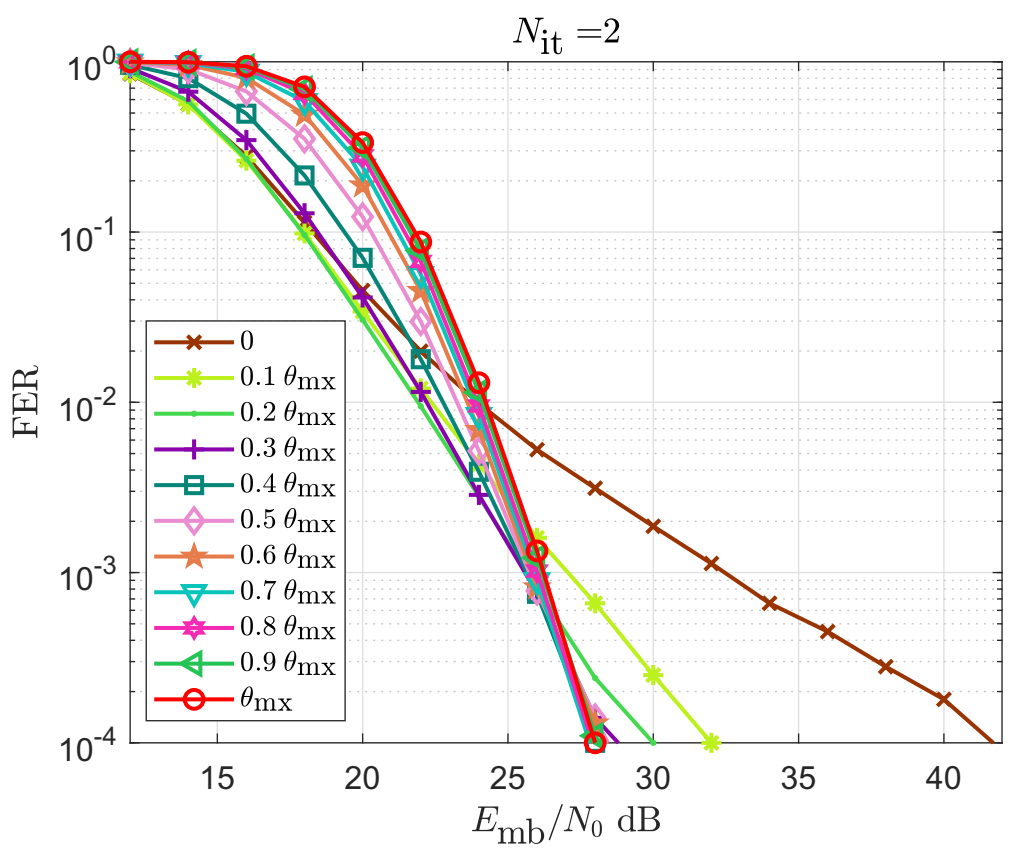

Figure 4.4: The effect of changing $\theta$ on the FER performance of an LDPC-coded SCMA systems with $N_{\text {it }}=2$.

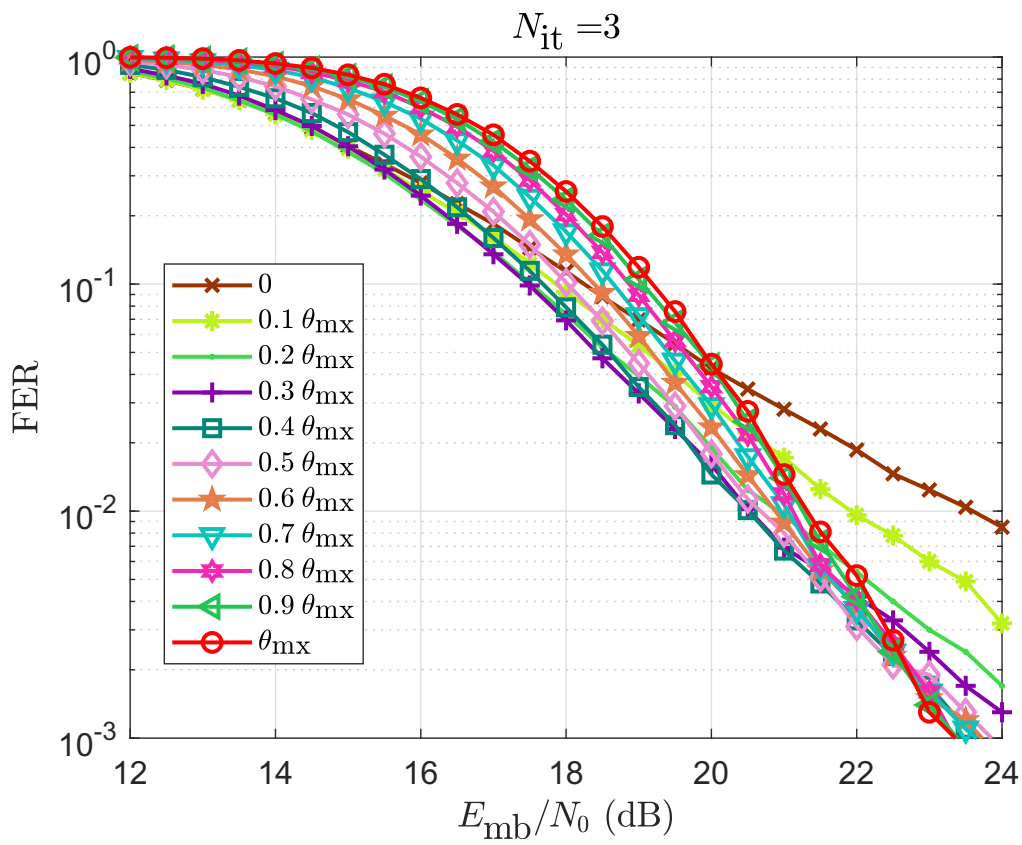

Figure 4.5: The effect of changing $\theta$ on the FER performance of an LDPC-coded SCMA systems with $N_{\text {it }}=3$. 


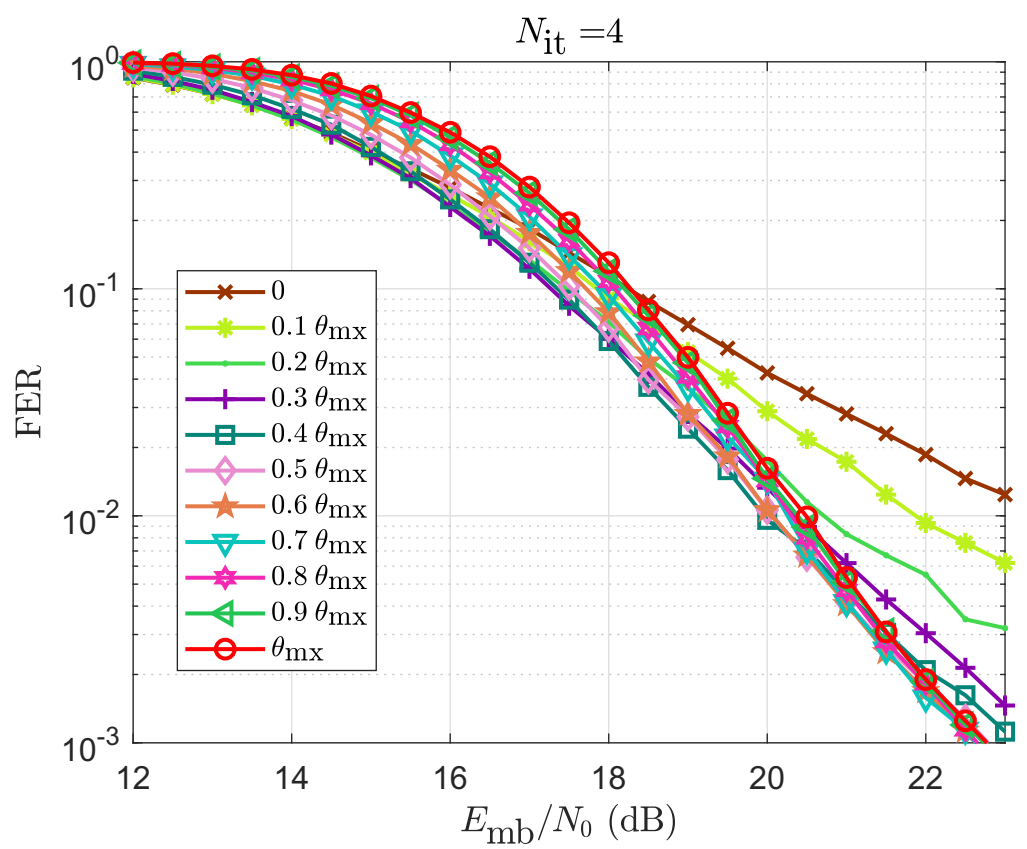

Figure 4.6: The effect of changing $\theta$ on the FER performance of an LDPC-coded SCMA systems with $N_{\text {it }}=4$.

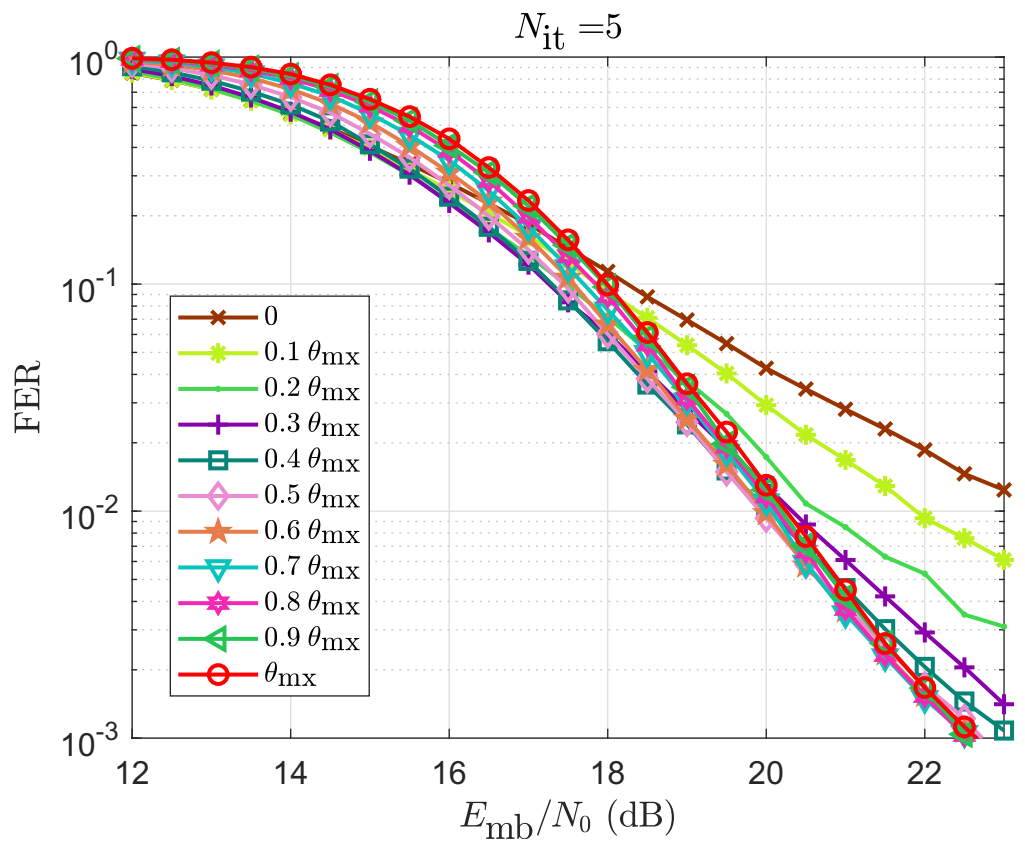

Figure 4.7: The effect of changing $\theta$ on the FER performance of an LDPC-coded SCMA systems with $N_{\text {it }}=5$. 
The results in Section 3.5 shows that the T16QAM constellation $\left(\theta=\theta_{\mathrm{mx}}\right)$ outperforms other previously published MdCs. However, from Fig. 4.3-Fig. 4.7, we see that for a target FER of $10^{-2}$, the $\theta=0$ option (an SNR-tolerant system), and the $\theta=0.5 \theta_{\mathrm{mx}}$ option with $N_{\mathrm{it}}=5$ (a delay-tolerant system) outperform the T16QAM constellation by about $7 \mathrm{~dB}$ and $0.5 \mathrm{~dB}$, respectively.

\subsection{Proposed Search Method}

As we discussed in Section 4.2.2, the value of the optimal rotation angle, $\theta_{\mathrm{opt}}$, changes for different SNRs and $N_{\text {it }}$. In order to find $\theta_{\text {opt }}$ at a certain SNR and for a given $N_{\mathrm{it}}$, one brute-force way is to perform an exhaustive search. Although from Section 4.2.2 the search space can be limited to the range of 0 to $\theta_{\mathrm{mx}}$, the exhaustive search is still cumbersome. In this section, using a second-order rational polynomial, and based on the Levenberg-Marquardt algorithm for nonlinear least squares curve-fitting problems [101], we propose an algorithm that finds a close-to-optimal angle, $\theta_{\text {copt }}$, with a much lower complexity than an exhaustive search.

The second-order rational-polynomial fit function is given by:

$$
\operatorname{fit}(\theta)=\frac{p_{2} \theta^{2}+p_{1} \theta+p_{0}}{\theta^{2}+q_{1} \theta+q_{0}}
$$

where $p_{0}, p_{1}, p_{2}, q_{0}$, and $q_{1}$ represent the fitting coefficients and to be determined. We select $N_{s}$ evenly-spaced $\theta$ samples, $\left\{\theta_{n}\right\}$, that lie in the range of 0 to $\theta_{\mathrm{mx}}$; we then evaluate $\operatorname{FER}\left(\theta_{n}\right)$ for each $\theta_{n}$ using Monte Carlo simulation, at a certain SNR and for a given $N_{\text {it }}$. Since there are 5 unknown coefficients in (4.14), we need at least 5 samples, i.e., $N_{s} \geq 5$. We then use the Levenberg-Marquardt algorithm for nonlinear least squares curve-fitting, which is described in detail in [101], to determine the coefficients in (4.14) that provide the best fit between $\left\{\operatorname{fit}\left(\theta_{n}\right)\right\}$ and $\left\{\operatorname{FER}\left(\theta_{n}\right)\right\}$ in the least squares sense. The higher the $N_{s}$, the more the fit will be close to the actual function and thus the more accurate the results, but at the expense of more complexity. We then find $\theta_{\text {copt }}=\arg \min _{\theta} \operatorname{fit}(\theta)$ as an approximation to the optimal rotation angle. In Algorithm 1, we provide a summary of our proposed method to find $\theta_{\text {copt }}$.

In Fig. 4.8 , for $E_{\mathrm{mb}} / N_{0}=17 \mathrm{~dB}$ and $N_{\mathrm{it}} \leq 5$, we show $\left\{\operatorname{FER}\left(\theta_{n}\right)\right\}$ versus $\left\{\theta_{n}\right\}$, 
Algorithm 1: Proposed search algorithm

1: Select $N_{s} \geq 5$ evenly-spaced $\theta$ samples, $\theta_{n}$, that lie in the range of 0 to $\theta_{\mathrm{mx}}$, i.e., $\theta_{n} \in\left[0, \theta_{\mathrm{mx}}\right], n \in\left\{1, \ldots, N_{s}\right\}$.

2: At a certain SNR and for a given $N_{\text {it }}$, evaluate $\operatorname{FER}\left(\theta_{n}\right)$ for each $\theta_{n}$ using the Monte Carlo simulation.

3: Fit a second order rational polynomial function (4.14) by finding its 5 coefficients from the collected samples $\left\{\theta_{n}\right\}$ and $\left\{\operatorname{FER}\left(\theta_{n}\right)\right\}$ in Steps 1 and 2 using the Levenberg-Marquardt algorithm for nonlinear least squares curve-fitting [101].

4: Find the minimum of the second order polynomial function found in Step 3.

5: Report the angle corresponding to the minimum value in Step 4 as $\theta_{\text {copt }}$.

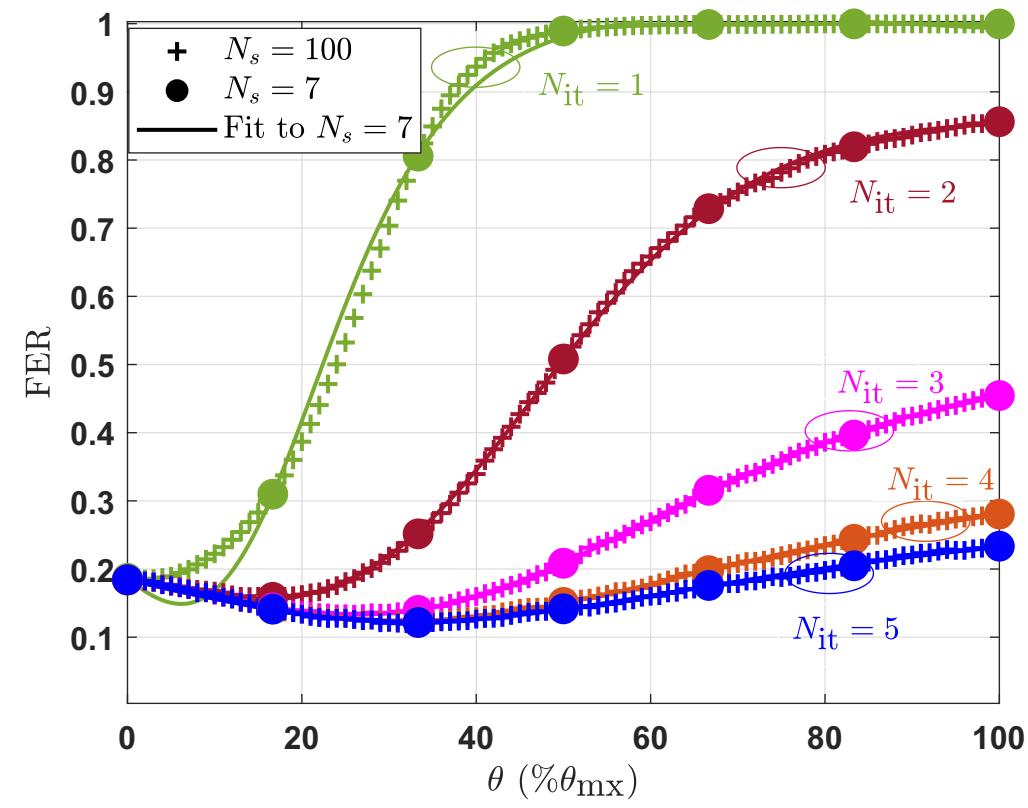

Figure 4.8: $\left\{\operatorname{FER}\left(\theta_{j}\right)\right\}$ versus $\left\{\theta_{j}\right\}$ for $N_{s}=100, N_{s}=7$, and the fitted second-order rational polynomial to the $N_{s}=7$ case, when $E_{\mathrm{mb}} / N_{0}=17 \mathrm{~dB}$ and $N_{\mathrm{it}} \leq 5$. 
which is expressed in terms of the percentage of $\theta_{\mathrm{mx}}$, when $N_{s}=100$ and $N_{s}=7$. We also depict the fitted second order rational polynomial fit to the $N_{s}=7$ case. We observe from Fig. 4.8 that $N_{\text {it }} \geq 2$ the fitted curve to $N_{s}=7$ also fits with $N_{s}=100$. Therefore, $\theta_{\text {copt }}$ can be found using only $N_{s}=7$ samples. For the scenario illustrated in Fig. 4.8, when $N_{\text {it }}=1$, the fitted curve to $N_{s}=7$ reports $\theta_{\text {copt }_{1}} \simeq 6.2 \%$, which corresponds to $\operatorname{FER}\left(\theta_{\operatorname{copt}_{1}}\right)=0.2007$, whereas the data from the $N_{s}=100$ case suggests that $\theta_{\text {copt }_{2}}=0$, which corresponds to $\operatorname{FER}\left(\theta_{\text {copt }_{2}}\right)=0.1864$. In other words, the fitted curve to $N_{s}=7$ resulted in only about $7 \%$ measurement error in terms of FER compared with $N_{s}=100$, but with much lower complexity.

In Fig. 4.9 , we show the effect of changing $E_{\mathrm{mb}} / N_{0}$ on $\theta_{\text {copt }}$ for different $N_{\mathrm{it}} \leq 5$ using the rational-polynomial fit to $N_{s}=7$. As we can see in Fig. 4.9 for a given $N_{\text {it }}$, $\theta_{\text {copt }}$ increases with increasing $E_{\mathrm{mb}} / N_{0}$. We also observe that the effect of increasing $\theta_{\text {copt }}$ with $E_{\mathrm{mb}} / N_{0}$ is more pronounced when $N_{\mathrm{it}} \geq 2$. In Fig. 4.10, we show the effect of changing $N_{\mathrm{it}}$ on $\theta_{\text {copt }}$ for $12 \leq E_{\mathrm{mb}} / N_{0} \leq 20$ using the rational-polynomial fit to $N_{s}=7$. As we can see in Fig. 4.10 for a given $E_{\mathrm{mb}} / N_{0}, \theta_{\text {copt }}$ increases with the increase in $N_{\text {it }}$. We also note from Fig. 4.10 that $\theta_{\text {copt }} \simeq 0$ for $E_{\mathrm{mb}} / N_{0}=12 \mathrm{~dB}$, and for other $E_{\mathrm{mb}} / N_{0}$ with $N_{\mathrm{it}}=1$.

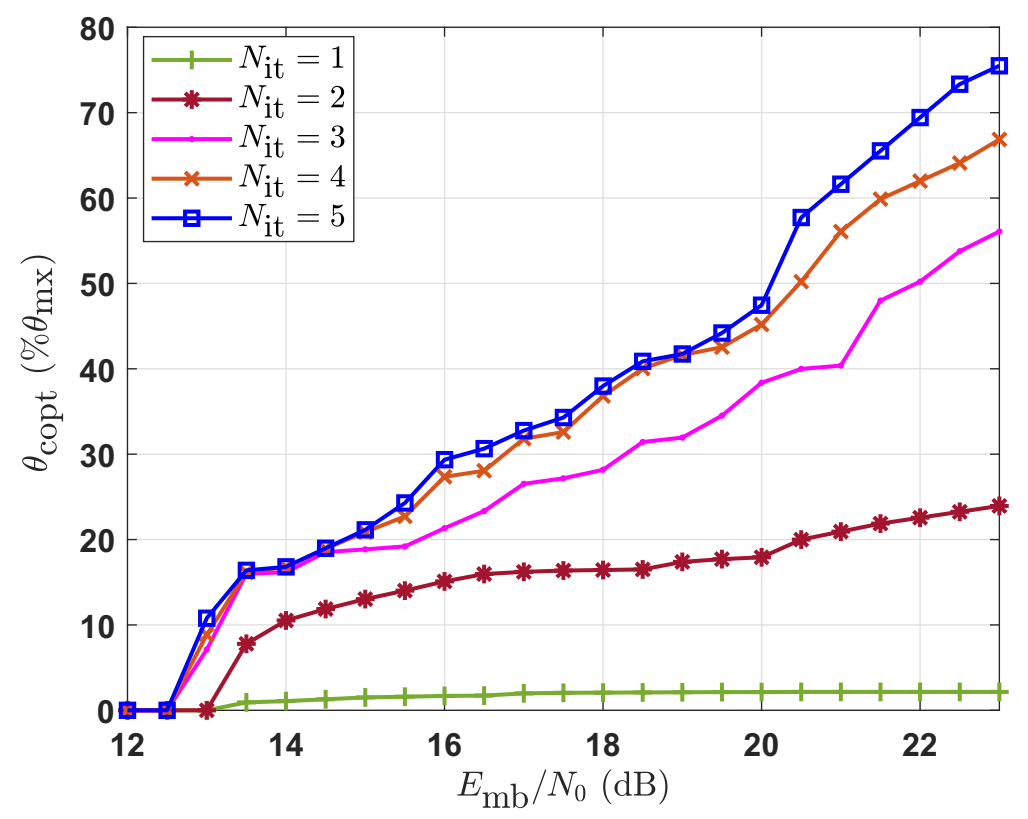

Figure 4.9: The effect of changing $E_{\mathrm{mb}} / N_{0}$ on $\theta_{\text {copt }}$ for different $N_{\text {it }} \leq 5$ using the rational-polynomial fit to $N_{s}=7$. 


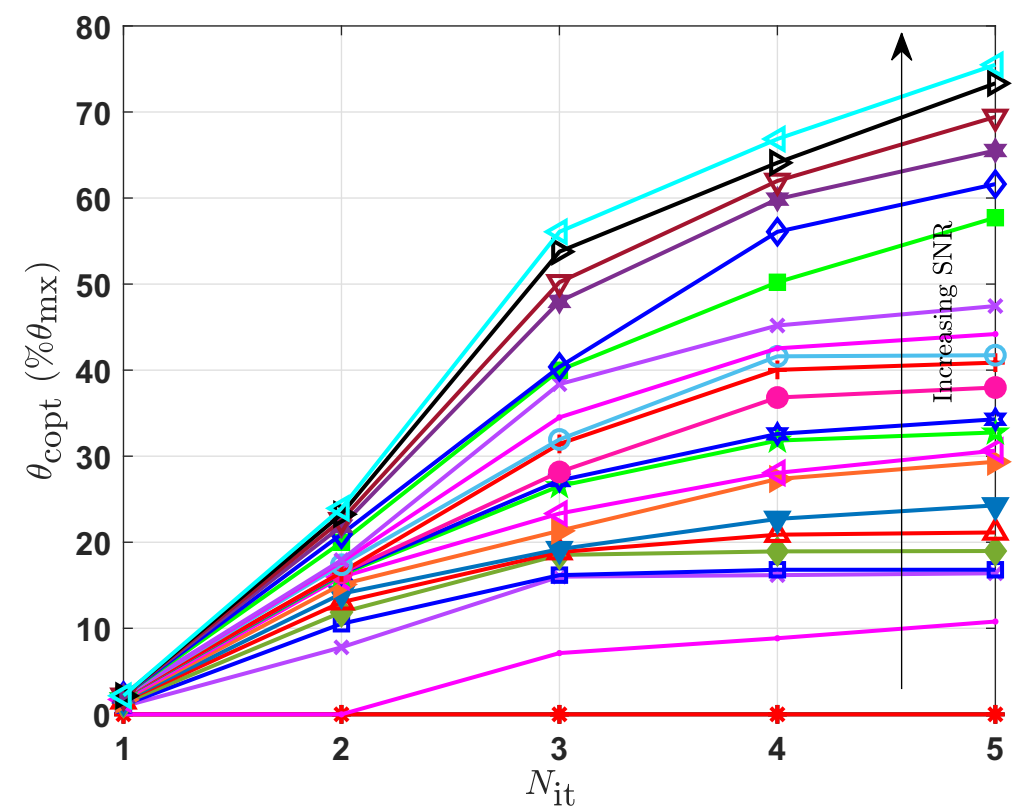

Figure 4.10: The effect of changing $N_{\text {it }}$ on $\theta_{\text {copt }}$ for $12 \leq E_{\mathrm{mb}} / N_{0} \leq 20$ using the rational-polynomial fit to $N_{s}=7$.

In Fig. 4.11-Fig. 4.15, we compare the FER performance of the SCMA system under study with respect to SNR at different $N_{\text {it }}$, with the 16 HQAM constellation $(\theta=$ $0)$, the T16QAM constellation $\left(\theta=\theta_{\mathrm{mx}}\right)$, and the proposed MdCs that correspond to $\theta=\theta_{\text {copt }}$ for different $E_{\mathrm{mb}} / N_{0} \mathrm{~s}$. As we see from Fig. $4.11-$ Fig. 4.15 , for an SNRtolerant system $\left(N_{\mathrm{it}}=1\right)$ and for low SNRs with $N_{\mathrm{it}} \geq 2, \theta_{\text {copt }}=0$ and thus the proposed MdC coincides with the 16HQAM constellation. On the other hand, we observe from Fig. 4.15 that for a delay-tolerant system with $N_{\text {it }}=5$, for a target FER of $10^{-2}$, the proposed MdC outperforms the T16QAM constellation by about $0.5 \mathrm{~dB}$. We also note from Fig. 4.11-Fig. 4.15 that $\theta_{\text {copt }}$ converges to $\theta_{\mathrm{mx}}$ at high SNRs. Between the two extremes, the gain of the proposed scheme is more noticeable, e.g., for $N_{\text {it }}=2$, our proposed scheme provides a gain of about $2 \mathrm{~dB}$ to achieve a target FER of $10^{-2}$.

For a certain $N_{\text {it }}$, we design an MdC that achieves the target FER at the lowest possible SNR. The designed MdCs result in substantial performance gains in comparison to the best known SCMA MdCs in the literature, especially in low-to-medium 


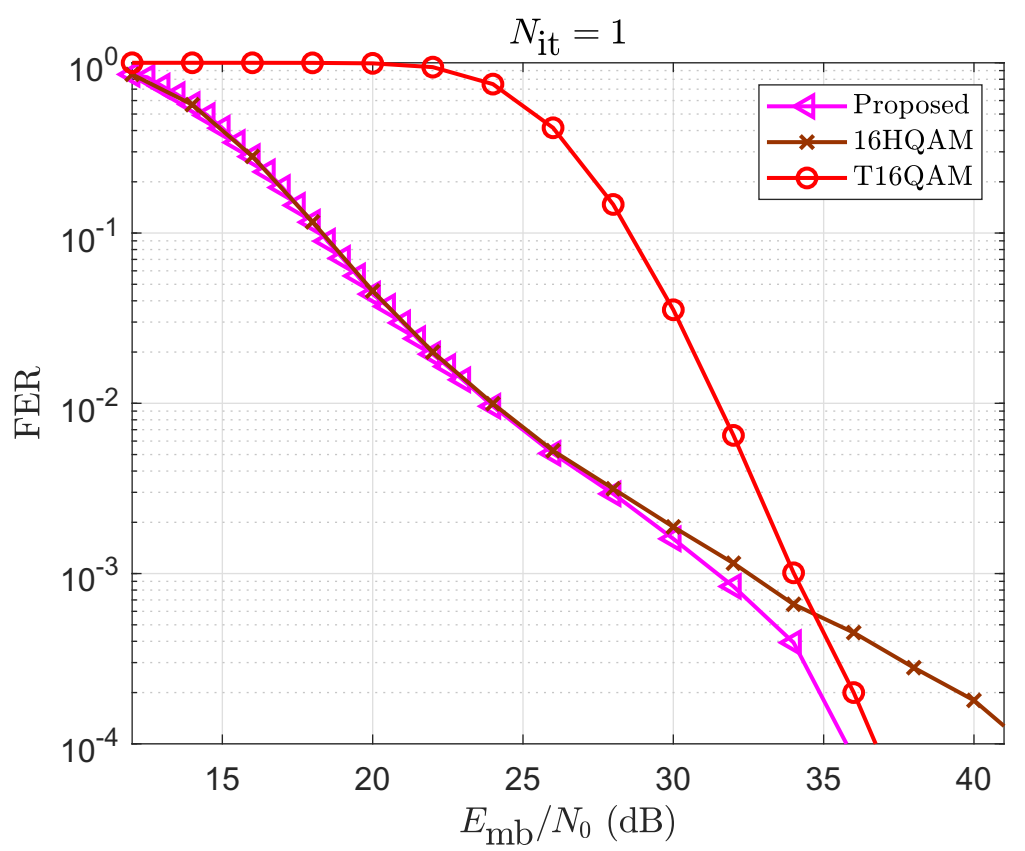

Figure 4.11: FER performance comparison of an SCMA system with the 16HQAM $(\theta=0)$, T16QAM $\left(\theta=\theta_{\mathrm{mx}}\right)$, and $\theta=\theta_{\text {copt }}$ for different $E_{\mathrm{mb}} / N_{0} \mathrm{~s}$ and $N_{\mathrm{it}}=1$.

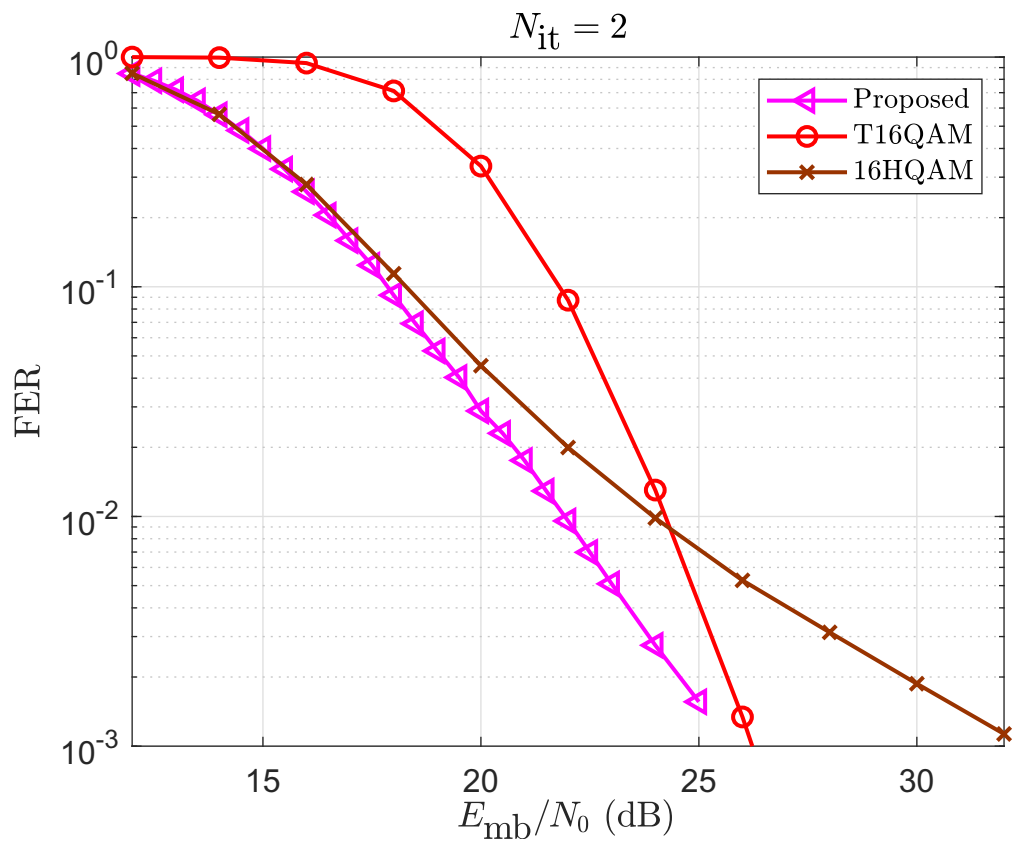

Figure 4.12: FER performance comparison of an SCMA system with the 16HQAM $(\theta=0)$, T16QAM $\left(\theta=\theta_{\mathrm{mx}}\right)$, and $\theta=\theta_{\text {copt }}$ for different $E_{\mathrm{mb}} / N_{0} \mathrm{~s}$ and $N_{\mathrm{it}}=2$. 


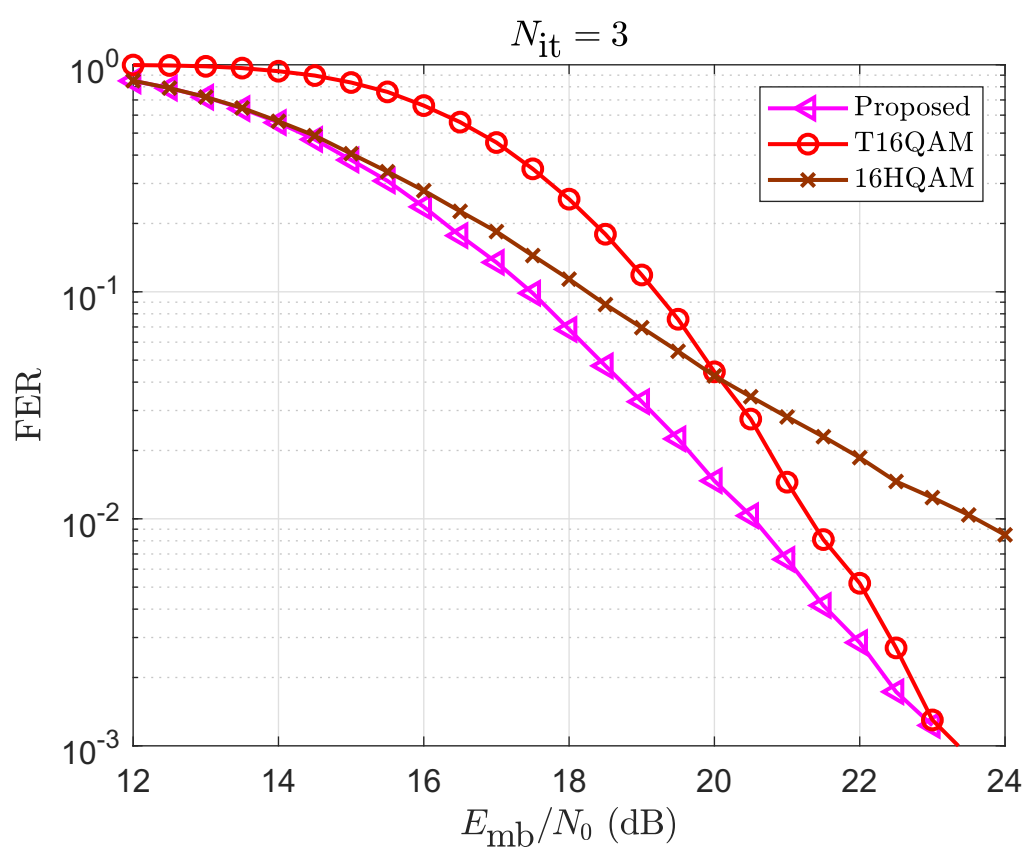

Figure 4.13: FER performance comparison of an SCMA system with the 16HQAM $(\theta=0)$, T16QAM $\left(\theta=\theta_{\mathrm{mx}}\right)$, and $\theta=\theta_{\text {copt }}$ for different $E_{\mathrm{mb}} / N_{0} \mathrm{~s}$ and $N_{\mathrm{it}}=3$.

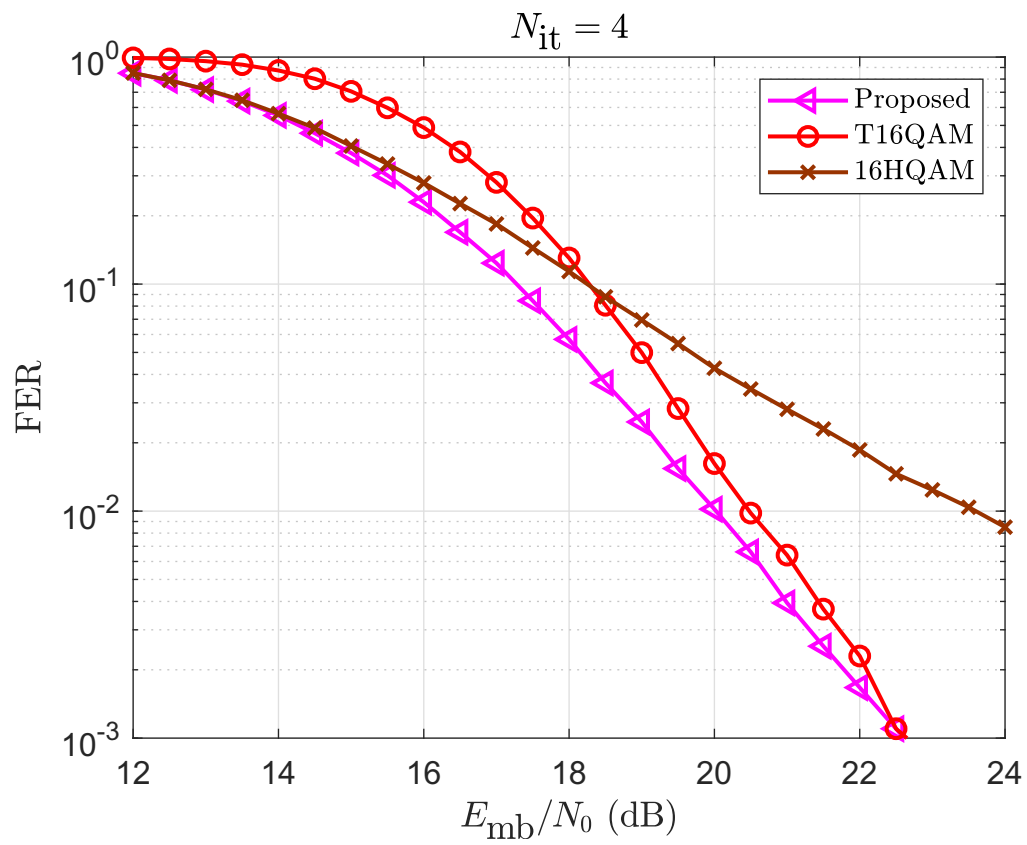

Figure 4.14: FER performance comparison of an SCMA system with the 16HQAM $(\theta=0)$, T16QAM $\left(\theta=\theta_{\mathrm{mx}}\right)$, and $\theta=\theta_{\text {copt }}$ for different $E_{\mathrm{mb}} / N_{0} \mathrm{~s}$ and $N_{\mathrm{it}}=4$. 
SNR regions when $N_{\text {it }} \leq 5$.

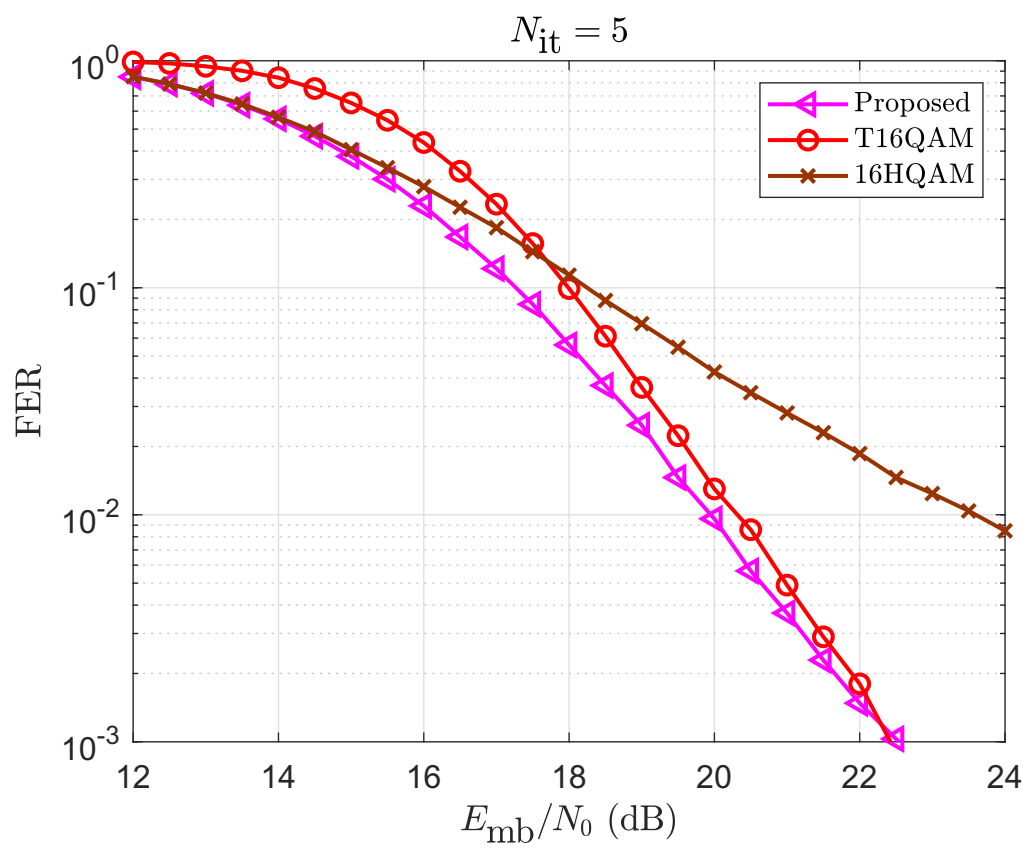

Figure 4.15: FER performance comparison of an SCMA system with the 16HQAM $(\theta=0)$, T16QAM $\left(\theta=\theta_{\mathrm{mx}}\right)$, and $\theta=\theta_{\text {copt }}$ for different $E_{\mathrm{mb}} / N_{0} \mathrm{~s}$ and $N_{\mathrm{it}}=5$.

\subsection{Conclusion}

We devised a new method for designing multidimensional constellations (MdCs), aiming to minimize the FER of a 5G-compliant LDPC-coded uplink SCMA system. Due to the sparse nature of SCMA, the widely-used message passing algorithm (MPA) is used for SCMA detection. The MPA performance is closely tied with the SNR operating region and the number of MPA iterations, $N_{\text {it }}$, and asymptotically approaches the optimal ML performance when $N_{\text {it }}$ goes to infinity. Also, it is known that the Gray-labelled MdCs that are constructed based on hypercubes perform well when used along with BICM as they obtain an equal error protection among their different bit levels. On the other hand, obtaining the closed-form expression of the FER in a coded SCMA system that is a function of the optimum rotation angle, the SNR operating region, and $N_{\mathrm{it}}$ is difficult. One brute-force way to seek for an optimum rotation angle is to perform an exhaustive search over the entire range of the rotation angle, $[0,2 \pi]$, that leads to a minimum FER. At a given SNR, for a certain $N_{\text {it }}$, and using the 
special properties of hypercubes, we limited the search space of the rotation angle to $[0, \pi / 4]$, and reduced the search complexity, which can still result in $\theta_{\text {opt }}$. We further decreased the complexity of the search by fitting a second order rational polynomial to a few measured FER samples, and were able to find a close-to-optimal rotation angle. Our proposed MdCs demonstrated substantial performance gains (as much as $2 \mathrm{~dB}$ ) in comparison to the best known SCMA MdCs in the literature, especially in low-to-medium SNR regions when $N_{\text {it }} \leq 5$. 


\section{SCMA Multiuser Detection}




\section{Chapter 5}

\section{SD-based Detection for Generalized SCMA Constellations}

\section{$5.1 \quad$ Introduction}

In an SCMA system, deploying a multiuser detection scheme that efficiently recovers the transmitted data from each user is a key issue. The complexity of the classical optimal maximum likelihood (ML) detection that is based on the exhaustive search over all possible codewords is high, and grows exponentially with the number of users. By exploiting the sparse structure of SCMA, the message passing algorithm (MPA) is widely used in literature to detect the SCMA codewords. However, MPA is not an optimal scheme, and the complexity of the conventional MPA grows exponentially with the number of users that occupy each RE.

Sphere decoding (SD) is an efficient way to reduce the complexity of classical ML detection. It prevents the exhaustive search over all possible codewords by limiting its search space to a hypersphere centered at the received vector. SD has been suggested by a number of authors as an effective detection algorithm in a variety of contexts. It is in fact shown in [107] that SD can be efficient in some signal to noise ratio (SNR) regions and small to moderate size problems. In the context of SCMA, a number of SD-based detection schemes has been proposed to reduce the complexity of the conventional MPA and/or to achieve a better performance. Instances of these schemes are provided in $[105,108-114]$ as we elaborate below.

In $[105,108,109], \mathrm{SD}$ is employed prior to MPA to reduce its search space and thus its complexity. The method devised in $[105,108]$ offers a tradeoff between performance and complexity. The SD-based MPA (SD-MPA) in [109] calculates the superimposed 
constellation point inside a spherical region. The radius of that region corresponds to the variance of additive white Gaussian noise (AWGN), which results in a reduction in the search space of MPA. However, the performance of SD-MPA in [109] degrades when the radius is small, and its complexity is high at low SNRs. In [110] a list SD-based detector is proposed for SCMA that achieves the optimal ML performance but with a comparable complexity to MPA. Inspired by the sparsity of SCMA codewords, an optimal SD-based detector is devised in [111] that has a lower complexity compared with the conventional SD. However, a complexity comparison with MPA is not reported in [111]. In [112], an SD-based detector is developed that obtains the MPA performance but at a lower computational cost, and in [113], SD is employed in a downlink SCMA system to reduce its detection complexity. By employing a minimum mean square error (MMSE) of the transmitted signal, an SD-based detection with joint list decoding algorithm for polar coded SCMA is proposed in [114] that has low complexity.

The different SD-based detectors in [105,108-114] pointed out above support only constellations with constant modulus and a subset of non-constant modulus constellations. More specifically, they can be applied only on constellations that are in the form of a product of a constant modulus constellation with a block-diagonal matrix. However, consider the 4-Beko constellation (Chapter 3.4) that is an irregular constellation with a non-constant modulus that was proposed in [68], and later used in the context of SCMA systems [61]. The results in Chapter 3.5 show that in some scenarios the 4-Beko constellation outperforms other known constellations. Moreover, the optimum SCMA constellation is still unknown. As such, the need for efficient detection schemes suitable for all types of constellations becomes necessary.

In this chapter, we develop a novel SD-based detection algorithm that can be applied on any constellations, and achieves the optimal ML performance. We refer to this SD-based detection as an improved SD (ISD). Similar to other SD-based methods, the proposed algorithm is based on a tree search. However, the number of floating-point operations (FLOPs) needed to calculate the metric at each layer of the tree in ISD is independent of the number of users. This substantially reduces the complexity of the proposed scheme. 


\subsection{Detection Problem Formulation}

In this chapeter, we only focus on uncoded SCMA systems, in which there is only one use of the channel. For notational brevity, we drop the subscript $i$ from (2.4). The uncoded uplink SCMA system in (2.4) can then be rewritten as

$$
\mathbf{y}=\sum_{k=1}^{K} \operatorname{diag}\left(\mathbf{h}_{k}\right) \mathbf{S}_{k} \tilde{\mathbf{x}}_{k}+\mathbf{w}
$$

We refer to each element of $\tilde{\mathbf{x}}_{k}$ as a layer that is sent over one RE of the $d_{v}$ REs designated to user $k$. Restated, each user has $d_{v}$ layers, and there are a total of $L=d_{v} K$ layers in the system. We define $\tilde{\mathbf{x}}=\left(\tilde{\mathbf{x}}_{1}^{\mathrm{T}}, \ldots, \tilde{\mathbf{x}}_{K}^{\mathrm{T}}\right)^{\mathrm{T}}=\left(\tilde{x}_{1}, \ldots, \tilde{x}_{L}\right)^{\mathrm{T}}$ as an $L$-dimensional vector containing the transmitted constellation points (symbols) of all layers that are selected from a constellation set $\mathcal{X} \subseteq \mathbb{C}^{L}$, with the alphabet size of $M$. The $d_{v}$ constellations points of $\tilde{\mathbf{x}}$ with indices, $x_{l} \in\left(\tilde{x}_{(k-1) d_{v}+1}, \ldots, \tilde{x}_{k d_{v}}\right), l \in\{1, \ldots, L\}$, correspond to user $k$, viz., the elements of $\tilde{\mathbf{x}}_{k}$.

To proceed with the proposed ISD detection scheme, we define the effective channel coefficients for user $k$ as $\mathbf{G}_{k}=\operatorname{diag}\left(\mathbf{h}_{k}\right) \mathbf{S}_{k}$, and rewrite the received vector $\mathbf{y}$ in (5.1) as

$$
\mathbf{y}=\mathbf{G x}+\mathbf{w},
$$

where $\mathbf{G}=\left(\mathbf{G}_{1}, \ldots, \mathbf{G}_{K}\right)$ denotes the $N \times L$ effective channel coefficient matrix for all users. The optimal ML detection problem for SCMA transmitted symbols is then

$$
\hat{\mathbf{x}}=\arg \min _{\mathbf{x} \in \mathcal{X}}\|\mathbf{y}-\mathbf{G} \mathbf{x}\|^{2},
$$

where $\hat{\mathbf{x}}=\left(\hat{x}_{1}, \ldots, \hat{x}_{L}\right)^{\mathrm{T}}$ represents the detected symbols. Because an exhaustive search implementation of the ML detector is highly complex, reduced-complexity search algorithms, such as SD, are desired. Moreover, since in overloaded SCMA the number of REs is less than the number of users, the number of rows in $\mathbf{G}$ is less than its number of columns, that is, (5.3) represents an under-determined system, so SD cannot be directly applied $[105,108-113]$. To transform (5.3) to a well-defined system of equations, $\mathbf{G}$ in (5.3) is written as $\mathbf{G}=\left[\mathbf{G}^{(1)} \mathbf{G}^{(2)}\right]$, where $\mathbf{G}^{(1)}$ is an $N$ by $N$ matrix representing the first $N$ columns of $\mathbf{G}$, and $\mathbf{G}^{(2)}$ is an $N$ by $L-N$ matrix representing the last $L-N$ columns of $\mathbf{G}$. We also write $\mathbf{x}$ in (5.3) as $\mathbf{x}=\left[\mathbf{x}^{(1)} \mathbf{x}^{(2)}\right]^{\mathrm{T}}$, where $\mathbf{x}^{(1)}$ and $\mathbf{x}^{(2)}$ denote the first $N$ elements, and the last $L-N$ elements of $\mathbf{x}$, respectively. 
The existing SD-based detectors for SCMA define

$$
\begin{aligned}
\tilde{\mathbf{y}} & =\left[\begin{array}{cc}
\mathbf{y}^{\mathrm{T}} & \mathbf{0}_{1 \times L-N}
\end{array}\right]^{\mathrm{T}}, \\
\tilde{\mathbf{G}} & =\left[\begin{array}{cc}
\mathbf{G}^{(1)} & \mathbf{G}^{(2)} \\
\mathbf{0}_{L-N \times N} & \mathbf{I}
\end{array}\right],
\end{aligned}
$$

where $\mathbf{0}_{A \times B}$ is a zero matrix of size $A$ by $B$, and $\mathbf{I}$ is an identity matrix of size $L-N$. The existing detectors in $[105,108-114]$ should optimally solve

$$
\hat{\mathbf{x}}=\arg \min _{\mathbf{x} \in \mathcal{X}}\left(\|\tilde{\mathbf{y}}-\tilde{\mathbf{G}} \mathbf{x}\|^{2}-\left\|\mathbf{x}^{(\mathbf{2})}\right\|^{2}\right),
$$

which is a well-defined system, and is equivalent to (5.3) for all types of constellation. However, the existing detectors [105,108-114] instead of evaluating (5.5) solve

$$
\hat{\mathbf{x}}=\arg \min _{\mathbf{x} \in \mathcal{X}}\|\tilde{\mathbf{y}}-\tilde{\mathbf{G}} \mathbf{x}\|^{2},
$$

which also represents a well-defined system, but the solution of (5.6) is identical to the solution in (5.3) only if $\left\|\mathbf{x}^{(2)}\right\|$ is constant, such as for constant-modulus constellations (e.g., QPSK). The detectors in [105, 108-114] also support a small subset of nonconstant modulus constellations, e.g., $M$-QAM, which can be written as a product of a constant modulus constellation with a block diagonal matrix, but cannot be applied to arbitrary constellations. In Section 5.2.1, by exploiting the sparsity structure of SCMA, we propose an SD-based branch-and-bound (B\&B) scheme, namely ISD, to solve (5.3) with reduced complexity that unlike the detectors in [105,108-114] achieves the optimal ML performance for all types of constellations.

The QR decomposition is commonly employed on the channel matrix prior to SD (e.g., [105, 108-113, 115, 116]) to obtain an upper-triangular matrix that facilitates the detection of each symbol. However, such a decomposition is required in every channel use, and its associated cost is burdensome [117]. From the sparse structure of SCMA we alleviate the need for the QR decomposition by assigning REs to users in a particular way that leads to an upper-triangular $\mathbf{S}$, and hence an upper-triangular G. That is, the leftmost $N$ by $N$ block of $\mathbf{S}$ should be an identity matrix. By labeling the REs from 1 to $N$, the allocation of REs to the first $N / d_{v}$ users (the first $N$ layers) can be performed as follows: User 1 is assigned to REs 1 to $d_{v}$, user 2 is assigned to REs $d_{v}+1$ to $2 d_{v}$, and in general, user $k$, for $k \leq N / d_{v}$, is assigned to REs $(k-1) d_{v}+1$ 
to $k d_{v}$. Thus, $\mathbf{S}$ is an upper-triangular matrix with non-zero elements on the diagonal. As a result, $\mathbf{G}$ is an upper-triangular matrix and SD can be directly applied. In cases when it is desirable to allocate specific users to specific REs, e.g., based on the channel condition, the same effect can be achieved by relabeling the users and the REs. It is important to note that this assignment is only required per resource allocation cycle and not per channel use. Moreover, the allocation of REs to the remaining $K-N / d_{v}$ layers will not affect the upper-triangular structure of $\mathbf{S}$ and can be performed in any order. Thus, the proposed ISD does not require the QR factorization.

\subsubsection{Proposed Detection Scheme}

In the following, we refer to layers with indices $l \in\{1, \ldots, N\}$, which correspond to the elements of $\mathbf{x}^{(1)}$, as Type I layers, and layers with indices $l \in\{N+1, \ldots, L\}$, which correspond to the elements of $\mathbf{x}^{(2)}$, as Type II layers ${ }^{1}$.

Motivated by [116] that considers the optimum detection of rank-deficient MIMO systems, we fix the elements of $\mathbf{x}^{(2)}$ and rewrite (5.3) as

$$
\hat{\mathbf{x}}^{(1)}=\arg \min _{\mathbf{x}^{(1)} \in \mathcal{X}^{(1)}}\left\|\mathbf{y}-\mathbf{G}^{(1)} \mathbf{x}^{(1)}-\mathbf{G}^{(2)} \mathbf{x}^{(2)}\right\|^{2},
$$

where $\hat{\mathbf{x}}^{(1)}$ denotes the first $N$ elements of $\hat{\mathbf{x}}$, and $\mathcal{X}^{(1)}=\left\{\left(x_{1}, \ldots, x_{N}\right)^{\mathrm{T}} \mid \mathbf{x} \in \mathcal{X}\right\}$. The detection problem in (5.7) represents a well-defined system, which can be performed for every choice of $\mathbf{x}^{(2)}$, and the ML solution in (5.3) is ultimately found for all types of constellations. Note that unlike the detection problem in (5.6), the proposed detection problem in (5.7) imposes no condition on $\mathbf{x}^{(1)}$ or $\mathbf{x}^{(2)}$. In other words, $\mathbf{x}$ is not required to have any certain structure, and thus the proposed scheme can be applied to all types of constellations.

The proposed ISD is based on a search over a tree with $L$ layers, where each layer corresponds to $x_{l}$. The tree search is done from layer $L$ down to layer 1 . The $d_{v}$ layers with the indices $l \in\left[(k-1) d_{v}+1, k d_{v}\right]$, correspond to user $k$, i.e., they are associated with the elements of $\mathbf{x}_{k}$. As such, there are up to $M$ branches for the layer with the index $l=k d_{v}$, and only one branch for layers with indices $l \in\left\{(k-1) d_{v}+1, \ldots, k d_{v}-1\right\}$. We show an example of the tree branching process for $K=6, d_{v}=2$, and $M=4$ in Fig. 5.1. As user 6 transmits its information over layers 12 and 11, we have

\footnotetext{
${ }^{1}$ Categorizing the elements in $\mathbf{x}$ as Type I and Type II layers allow us to treat the rank-deficient SCMA detection problem as several full-rank problems.
} 
four branches at layer 12 and only one at layer 11, corresponding to $x_{12}$ and $x_{11}$, respectively. As mentioned in Section 5.2, we fix the elements of $\mathbf{x}^{(2)}$ and solve (5.7), which in the tree search, corresponds to the following: For each path associated with Type II layers, i.e., $l \in\{N+1, \ldots, L\}$, we evaluate a partial metric at each branch of Type I layer of the tree, i.e., $l \in\{1, \ldots, N\}$. This metric is based on the partial metrics that are already found in the prior layers. Inspired by SD B\&B, the partial metric at each Type I layer $l$ is defined as

$$
\mu_{l}\left(\mathbf{x}_{l}^{L}\right)=\sum_{i=l}^{N}\left|y_{i}-\sum_{j=i}^{N} g_{i, j}^{(1)} x_{j}^{(1)}-\sum_{j=1}^{L-N} g_{i, j}^{(2)} x_{j}^{(2)}\right|^{2},
$$

where $\mathbf{x}_{l}^{L}=\left(x_{l}, \ldots, x_{L}\right)^{\mathrm{T}}$, and $g_{i, j}^{(1)}, g_{i, j}^{(2)}, x_{j}^{(1)}$, and $x_{j}^{(2)}$ represent each element of $\mathbf{G}^{(1)}$, $\mathbf{G}^{(2)}, \mathbf{x}^{(1)}$, and $\mathbf{x}^{(2)}$, respectively. The detection problem is to find the value of $\mathbf{x}_{1}^{L}$ that minimizes $\mu_{l}\left(\mathbf{x}_{1}^{L}\right)$. From the sparsity of SCMA, the number of non-zero elements in each row of $\mathbf{G}$ is $d_{c}$, with their positions identified by the indices of $1 \mathrm{~s}$ in each row of $\mathbf{F}$ (Chapter 2). Let $\mathcal{S}_{l}=\left\{j \mid f_{l, j}=1\right\}$, with cardinality $d_{c}=\left|\mathcal{S}_{l}\right|$, denote the positions of $1 \mathrm{~s}$ in each row of $\mathbf{F}$. The metric in (5.8) can be calculated recursively as

$$
\mu_{l}\left(\mathrm{x}_{l}^{L}\right)=\mu_{l+1}\left(\mathrm{x}_{l+1}^{L}\right)+\left|y_{l}-\sum_{j \in \mathcal{S}_{l}} g_{l, j} x_{j}\right|^{2}
$$

where $\mu_{l+1}\left(\mathbf{x}_{l+1}^{L}\right)$ indicates the metric calculated prior to layer $l$, i.e., at layer $l+1$. The principle of SD entails finding $\mathbf{x}_{1}^{L}$ that falls within a hypersphere of radius $d$ from the received vector. In ISD, that corresponds to finding $\mathbf{x}_{1}^{L}$ such that $\mu_{1}\left(\mathbf{x}_{1}^{L}\right) \leq d^{2}$. Since the partial metrics always increase the deeper into the tree as the search continues, if the partial metric $\mu_{l}\left(\mathrm{x}_{l}^{L}\right)$ exceeds $d^{2}$, then there is no point descending further along the current path. As such, the subtree emanating from the current layer can be pruned. The radius $d$ can be initially chosen as [105,118], $d_{0}^{2}=2 N_{0} \beta L-\mathbf{y}^{\mathrm{H}}(\mathbf{I}-$ $\left.\mathbf{G P}^{-1} \mathbf{G}^{\mathrm{H}}\right) \mathbf{y}$, where $\mathbf{P}=\tilde{\mathbf{G}}^{\mathrm{H}} \tilde{\mathbf{G}}, \tilde{\mathbf{G}}$ is given in (5.4), and $\beta \geq 1$ is chosen to assure that a candidate solution is captured. The value of $d$ is reduced during the tree search whenever a layer with a smaller metric is found. That is, when $\mu_{L}\left(\mathrm{x}_{1}^{L}\right)<d, d$ is updated and is set to $\mu_{L}\left(\mathbf{x}_{1}^{L}\right)$ [115] to ensure the ML solution is found. Note that Type II layers are just for keeping the track of that path of the tree to be explored and are never pruned. 


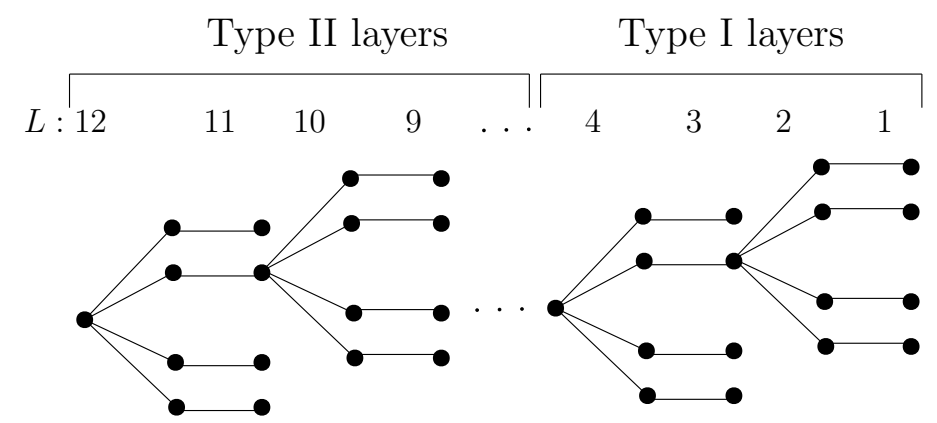

Figure 5.1: An example of the tree branching process.

\subsubsection{Reduced-complexity ISD}

The average complexity of an SD-based detector is commonly measured in terms of the average number of visited layers multiplied by the number of FLOPs required to calculate the metric at each layer of the tree, e.g., [105]. From (5.9), the number of real additions (RAs) and real multiplications (RMs) at each Type I layer is proportional to $d_{c}$. By exploiting the sparsity of $\mathbf{G}$, the complexity of ISD can be reduced by calculating the metric in (5.9) in a more efficient way with fewer RAs and RMs. Let $y_{l}$ and $g_{l, j}, l \in\{1, \ldots, N\}$, denote each element of $\mathbf{y}$ and $\mathbf{G}$, respectively. For Type I layers, by defining $z_{l, l}\left(\mathbf{x}_{l}^{L}\right)=y_{l}-\sum_{j=l}^{L} g_{l, j} x_{j}$, as $y_{l}$ with the effects of the symbols from layers $l$ and above removed, we can recursively find the rightmost term of (5.9) as

$$
z_{l, l}\left(\mathbf{x}_{l}^{L}\right)=z_{l, l+1}\left(\mathbf{x}_{l+1}^{L}\right)-g_{l, l} x_{l}
$$

The metric at layer $l$ of the tree search is then

$$
\mu_{l}\left(\mathbf{x}_{l}^{L}\right)=\mu_{l+1}\left(\mathbf{x}_{l+1}^{L}\right)+\left|z_{l, l}\left(\mathbf{x}_{l}^{L}\right)\right|^{2}
$$

Because there is only one non-zero element in each column of $\mathbf{G}, z_{l, l}\left(\mathbf{x}_{l}^{L}\right)$ in $(5.10)$ is updated for only one value of $l$ at each layer. From (5.10)-(5.11), ISD requires 6 RAs and 6 RMs to calculate the metric for each Type I layer. That is, the number of FLOPs at each layer of the tree search is fixed and is independent of $d_{c}$. This is unlike the existing SD-based methods in the context of SCMA, and reduces the complexity of ISD. Further, as mentioned in Section 5.2.1, no metric is calculated in Type II layers. Let $N_{v_{1}}$ denote the average number of visited layers for Type I layers. In Table 5.1, we provide the average number of RAs and RMs in the proposed ISD 
Table 5.1: Average complexity of ISD and MPA

\begin{tabular}{llc}
\hline & ISD & Log-MPA \\
\hline RAs & $6 N_{v_{1}}$ & $M N d_{c}\left(M^{d_{c}-1}\left(4 d_{c}-2+N_{\mathrm{it}}\left(2+\frac{1}{M}\right)\right)+N_{\mathrm{it}}\left(2-\frac{1}{d_{v}}\right)+5\right)$ \\
$\mathrm{RMs}$ & $6 N_{v_{1}}$ & $M N d_{c}\left(4 d_{c} M^{d_{c}-1}+5\right)$ \\
\hline
\end{tabular}

and Log-MPA (Appendix C).

\subsection{Performance Evaluation}

In this section, numerical results are provided to compare the performance of the proposed ISD scheme with Log-MPA (e.g., [119]) and ML for the following scenarios (Case 1 and Case 2 in Chapter 3): 1) Uncoded fading where each user observes the same channel coefficients over their $d_{v}$ REs (FSC), and 2) uncoded fading where each user observes independent channel coefficients over their $d_{v}$ REs (FIC). We set $K=6$, $N=4$, and $d_{v}=2$, with the user-to-RE indicator matrix in (2.3). We also use a single antenna at the transmitter of all users and at the receiver.

The 4-Beko constellation is an irregular constellation with a non-constant modulus that was proposed in [68], and later used in the context of SCMA systems as described in Section 3.4. The results in Section 3.5 show that in some scenarios 4Beko outperforms other known constellations. Yet, the existing SD-based schemes cannot be applied to the 4-Beko constellation. To confirm that the proposed ISD achieves the ML performance for all types of constellations, we compare the performance of uncoded SCMA systems that use the 4-Beko constellation with different detection schemes, and operate over FSC and FIC in Fig. 5.2. The T16QAM constellation was introduced in [30] and described in Section 3.4. We depict the performance comparison of SCMA systems that employ the T16QAM constellation with different detection schemes over FSC and FIC in Fig. 5.3. We use 5 MPA iterations for the 4-Beko constellation in Fig. 5.2, and 12 MPA iterations for T16QAM in Fig. 5.3.

From both Fig. 5.2 and Fig. 5.3, we observe that the proposed ISD achieves the ML performance. On the other hand, we see from Fig. 5.2 that MPA with 5 iterations approaches ML. Furthermore, Fig. 5.3 indicates that MPA requires 12 iterations to 


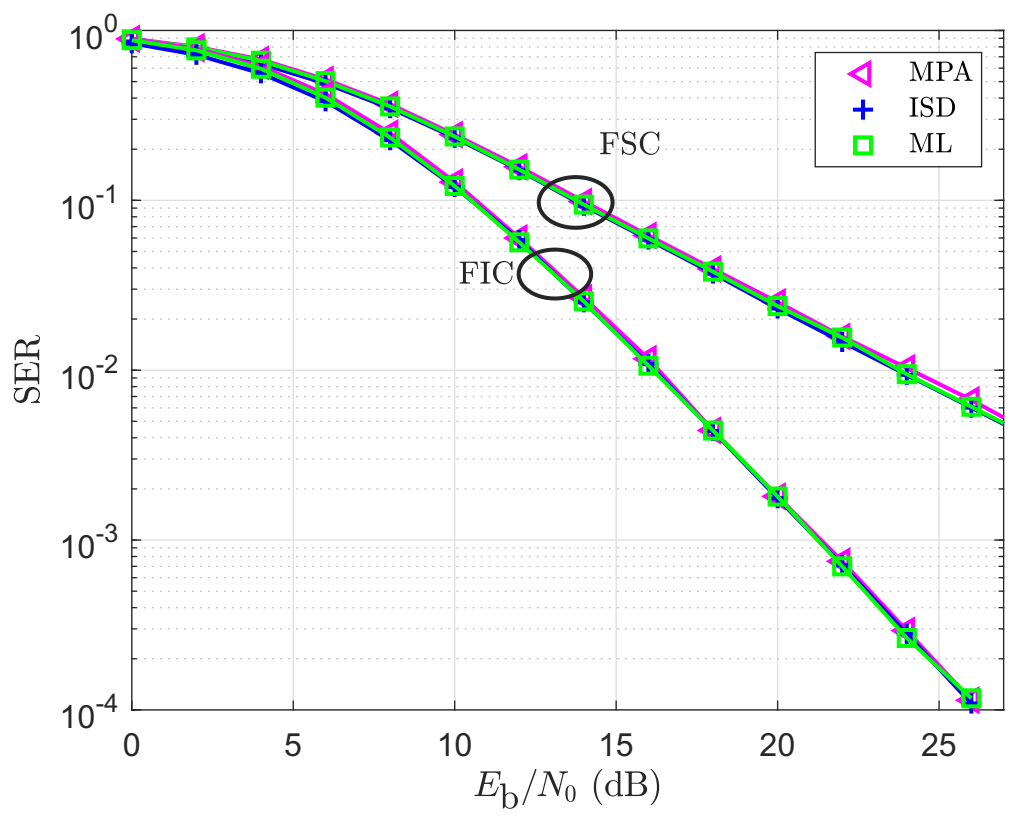

Figure 5.2: Performance of uncoded SCMA systems over FSC and FIC. All users employ the 4-Beko constellation.

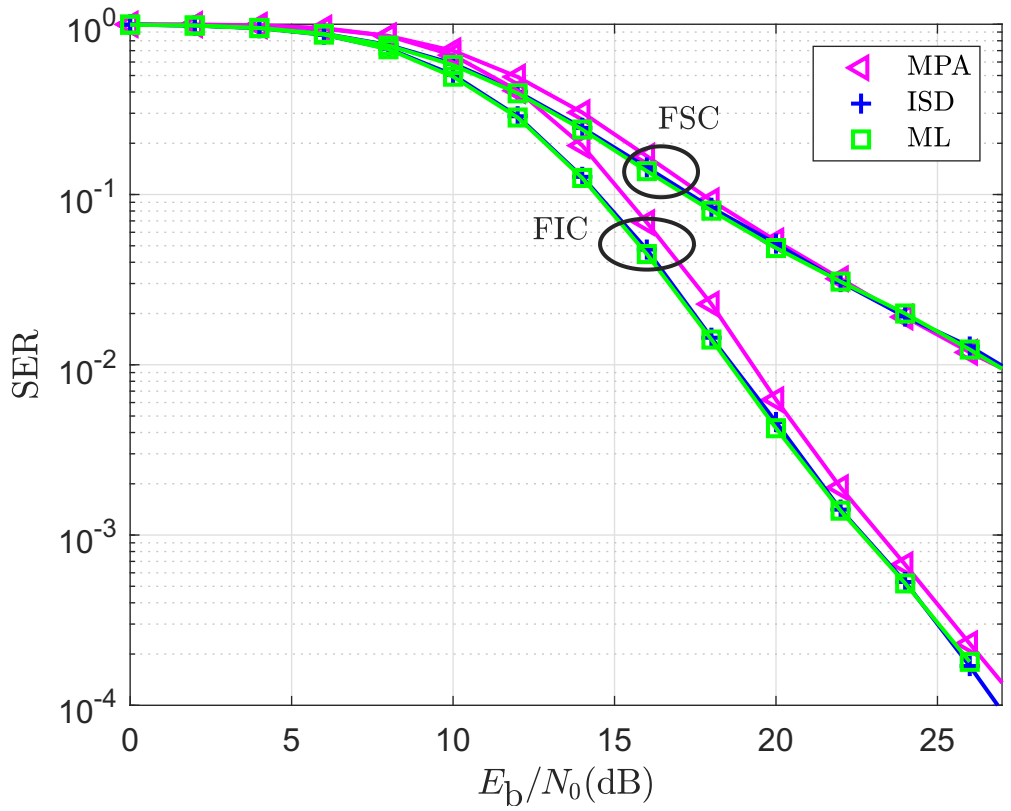

Figure 5.3: Performance of uncoded SCMA systems over FSC and FIC. All users employ the T16QAM constellation. 
approach the ML performance at high SNRs in the FSC case. Over FIC, MPA is off by about $0.5 \mathrm{~dB}$ compared with the proposed scheme.

In Fig. 5.4, we compare the average complexity of the proposed ISD with the complexity of commonly-used Log-MPA for an uncoded SCMA with the widely-used constant modulus constellation [1] over FSC. Moreover, we compare the average com-

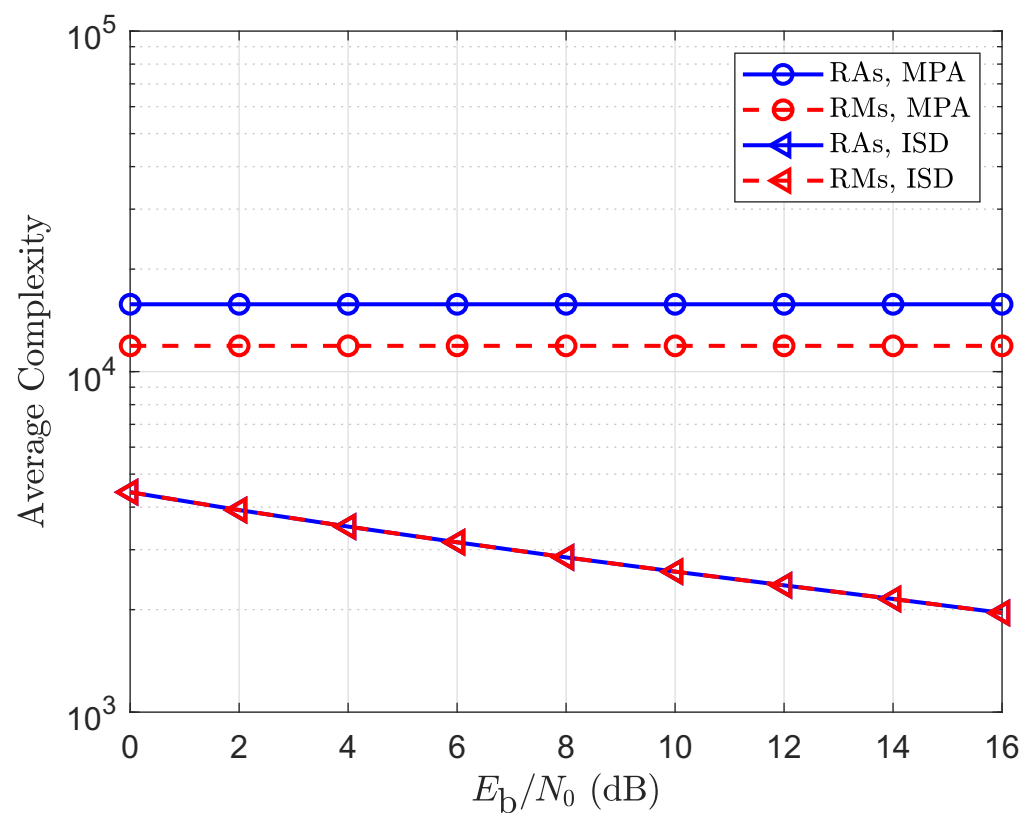

Figure 5.4: Average RAs and RMs in ISD and MPA with the constellation in [1].

plexity of the proposed ISD with Log-MPA over FIC when the 4-Beko constellation is employed in Fig. 5.5, and when the T16QAM constellation is employed in Fig. 5.6. All comparisons are in terms of RAs and RMs provided in Table 5.1 for ISD and Log-MPA. It is clear that in the scenarios depicted in Fig. 5.4-Fig. 5.6, the average complexity of the proposed ISD is much lower than MPA. We also note from Fig. 5.4-Fig. 5.6 that the average complexity of ISD is a function of SNR. This is because $d_{0}$ is smaller, and $d$ is reduced more quickly at higher SNRs, resulting in fewer visited layers. Furthermore, SD can be operated in parallel to reduce latency while maintaining low complexity [120]. 


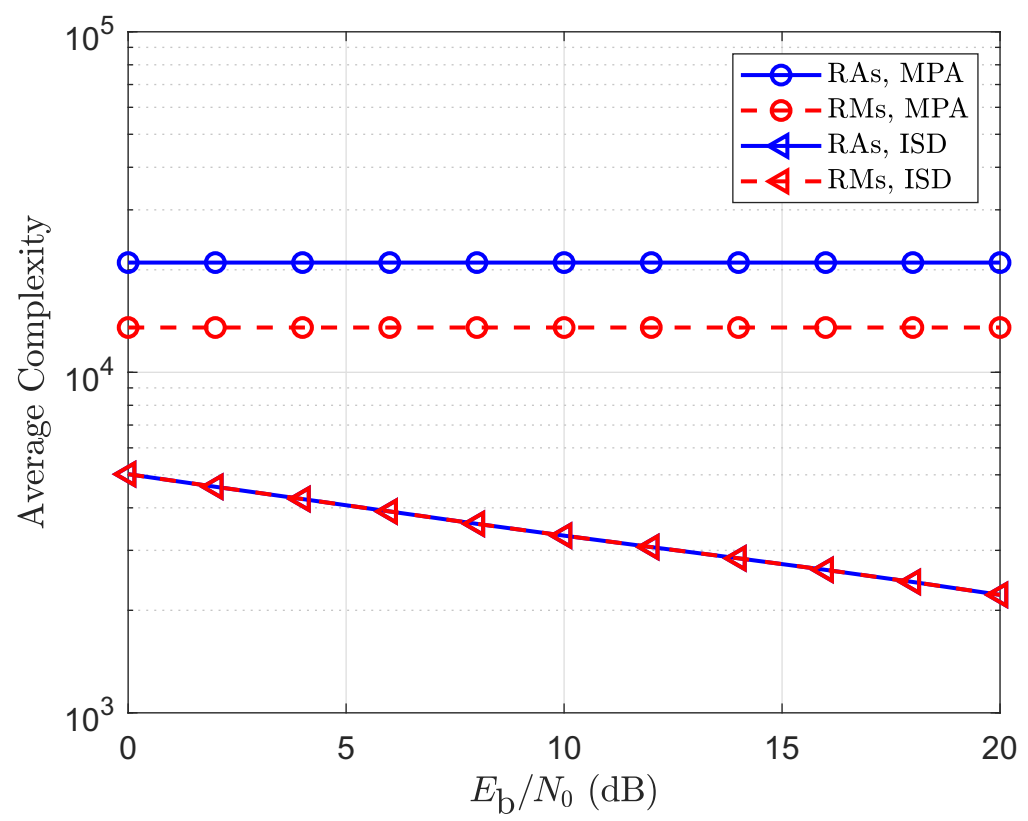

Figure 5.5: Average RAs and RMs in an uncoded SCMA system with the 4-Beko constellation.

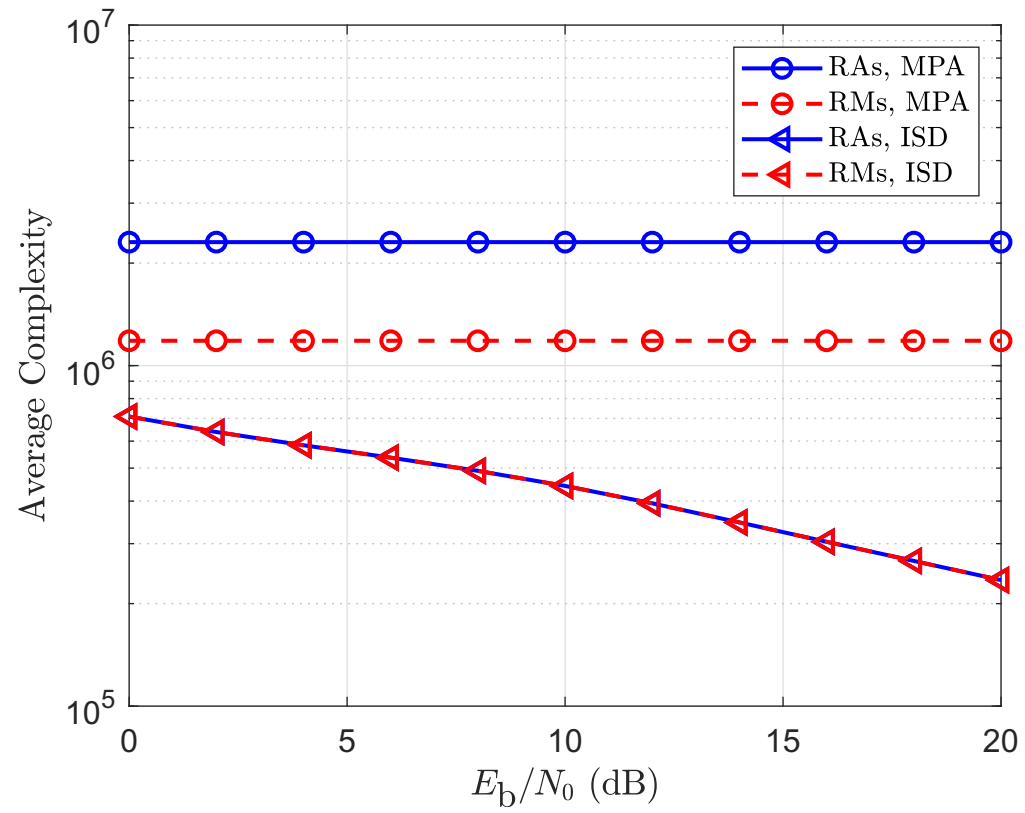

Figure 5.6: Average RAs and RMs in an uncoded SCMA system with the T16QAM constellation. 


\subsection{Conclusion}

We developed an improved SD-based detection scheme for uplink SCMA, which, unlike the existing SD-based schemes that can only be applied to a certain class of SCMA constellations, is able to achieve the optimal ML performance for all types of constellations. To overcome the rank deficiency problem of the SCMA channel matrix, we fixed a portion of the transmitted symbols and obtained an optimal detection problem that is equivalent to the original SCMA detection problem for any arbitrary regular or irregular constellations. Moreover, due to the sparse nature of SCMA, the partial metric at each layer is evaluated in such a way that is independent of users assigned to each RE $\left(d_{c}\right)$. This, in turn, reduces the average complexity of ISD. Simulation results demonstrated that the proposed ISD achieves the ML performance for different SCMA constellations with low computational complexity. 


\section{Chapter 6}

\section{Summary and Future Research Directions}

In this chapter, we first summarize this thesis and then provide some insights for the future research.

\subsection{Summary}

Since SCMA systems are among the potential candidates for uplink machine-type services, the focus of this thesis was on uplink SCMA systems. We described different elements of an SCMA uplink system model in Chapter 2. We then explored two main aspects of uplink SCMA systems, namely, multidimensional constellation design and detection techniques, in Chapter 3-Chapter 5.

As SCMA users map their incoming bits to multidimensional constellations (MdCs), designing good MdCs plays an important role in their performance. In Chapter 3, we highlighted and reviewed the key performance indicators (KPIs) of MdCs that should be considered in their design process under various channel scenarios. We also provided an extensive survey on different existing constellations over various channel scenarios. It is assumed that the CSI is not available at the transmitter. We use the widely-used near-optimal Log-MPA for the multiuser detection, and perform the multiuser detection and the turbo decoding separately in a concatenated manner. All turbo-coded comparisons are performed for BICM. Simulation results confirm that MdCs that satisfy KPIs of a certain channel scenario outperform other MdCs in that scenario. Moreover, the BER performance of uncoded systems, and the performance of the coded systems are tied to their bit-labeling. The performance of the systems also depends on the behavior of the multi-user detector at different SNR regions. 
It is known that the Gray-labelled MdCs that are constructed based on hypercubes perform well when used along with BICM as they obtain an equal error protection among their different bit levels. From the results in Chapter 3, we noticed that the T16QAM and 16-Bao constellations, which outperform other constellations in most channel conditions, are both based on the rotation of hypercubes. Due to the attractive features of hypercubes, in Chapter 4, we construct MdCs that are based on a unitary rotation of a hypercube by a certain rotation angle. Due to the sparse nature of SCMA, the widely-used MPA is used for SCMA detection. The MPA performance is closely tied with the SNR operating region and the number of MPA iterations, $N_{\text {it }}$, and asymptotically approaches the optimal ML performance when $N_{\text {it }}$ goes to infinity. On the other hand, obtaining the closed-form expression of the FER in a coded SCMA system that is a function of the optimum rotation angle, the SNR operating region, and $N_{\text {it }}$, is difficult. One brute-force way to seek for an optimum rotation angle is to perform an exhaustive search over the entire range of the rotation angle, $[0,2 \pi]$, that leads to a minimum FER. At a given SNR, for a certain $N_{\text {it }}$, and using the special properties of hypercubes, we limited the search space of the rotation angle to $[0, \pi / 4]$, and reduced the search complexity, which can still result in $\theta_{\text {opt }}$. We further decreased the complexity of the search by fitting a second order rational polynomial to a few measured FER samples, and were able to find a close-to-optimal rotation angle. Our proposed MdCs demonstrated substantial performance gains (as much as $2 \mathrm{~dB}$ ) in comparison to the best known SCMA MdCs in the literature, especially in low-to-medium SNR regions when $N_{\text {it }} \leq 5$.

In an SCMA system, deploying a multiuser detection scheme that efficiently recovers the transmitted data from each user is a key issue, and SD is an efficient way to reduce the complexity of classical ML detection. The existing SD-based detectors support only constellations with constant modulus and a subset of non-constant modulus constellations. More specifically, they can be applied only on constellations that are in the form of a product of a constant modulus constellation with a blockdiagonal matrix. However, the results in Chapter 3 show that in some scenarios the 4-Beko constellation, which is an irregular constellation, outperforms other known constellations. In Chapter 5, we develop an SD-based detection algorithm, namely ISD, that can be applied on any constellations, and achieves the optimal ML performance. Similar to other SD-based methods, the proposed algorithm is based on a tree search. However, the number of FLOPs needed to calculate the metric at each 
layer of the tree in ISD is independent of the number of users. This substantially reduces the complexity of the proposed scheme.

\subsection{Potential Future Research Directions}

The work presented in this thesis can be extended in different ways. In this section, we provide some future research directions.

\subsubsection{Multidimensional Constellations for SCMA Systems}

Some possible research directions in the line of MdCs for SCMA systems are as follows:

- It is assumed that CSI is not available at the transmitter. In some scenarios it is essential to obtain CSI at the transmitter. The impact of total CSI or partial CSI at the transmitter on the behaviour of MdCs can be investigated. For example, it is likely that the user-specific 2-dimensional rotations pointed out in Section 3.2 are useful when CSI is available at the transmitter.

- It is assumed that each user and the receiver are accompanied with a single transmit and a single receive antenna, i.e., SISO-SCMA. Alternatively, employing multiple transmit and receive (MIMO) antennas can improve the system performance by exploiting the spatial domain. As such, the effect of MIMO antennas, i.e., MIMO-SCMA (e.g., [121,122]) on the performance MdCs can be studied.

- All the comparisons are made for BICM. It is shown in the literature that for some applications (e.g., [123]) multilevel coding (MLC) [124] exhibits some gain over its BICM counterpart. Studying the KPIs for MLC is a possible research direction.

- One of the main challenges in SCMA systems is the receiver complexity that inherits from the detection of all active users [41]. In Chapter 3-4, the widelyused non-binary MPA is used as the multiuser detection. The impact of the choice of constellation when other lower complexity detectors (e.g., [105, 112, 125-133]) are used, should be further studied. 
- All the comparisons in this thesis have performed for a regular user-to-RE mapping matrix. It was also shown in [134], that a regular user-to-RE allocation is advantageous. Nevertheless, in a regular SCMA structure, it is not possible to serve different users with various requirements. Thus, studying the effect of an irregular SCMA structure, whereby users are not forced to occupy only a fixed number of REs, e.g., [135-137], is a possible research direction.

- Mathematical analysis of the error performance of constellations provides more indication on their performance in different SNR regions. In [103,138-142], the performance of some of the constellations has been analyzed. The performance analysis of different constellations under different scenarios is a possible research direction.

- The KPIs of designing MdCs for other channel models that are out of the scope of the current work can be explored.

- Obtaining the relationship between the KPIs that we have obtained for SCMA systems with the KPIs of single-user systems over different scenarios can be considered.

- The KPIs that are given for each specific scenario can be considered in the design process of new MdCs over each scenario.

- In Chapter 4, a combination of two Givens rotation with the same rotation angle along each dimension was considered to design new MdCs. This is not necessarily optimum as it may be possible to find better MdCs using different rotation angles along each dimension. With a 4-dimensional constellation, there are six Givens rotation angles, of which two have no effect. As such, a 4dimensional optimization technique is needed to either prove or disprove that using the same rotation angle is sufficient.

- It is also possible to use different rotation angles, and subsequently different Givens rotations, for different users and the benefit of doing so should be studied.

\subsubsection{Dynamic Modulation and Coding}

Motivated by the results of Chapter 3 and Chapter 4 , since the behavior of MdCs changes with the dynamic of the channel, the following can be done: 
- To design a constellation that can be applied to various channel conditions by considering the KPIs for different scenarios. This can be performed by formulating a multi-objective optimization problem.

- To develop a dynamic system that designs constellations based on the channel condition. This requires CSI at the transmitter, however, as in most typical scenarios the channel is slowly varying, the channel estimation does not need to be obtained on a frequent basis.

- To investigate different ways to efficiently perform the task of obtaining CSI at the transmitter.

- To develop a dynamic system that designs its modulation and coding scheme (MCS) based on the channel condition.

- The system can then be extended for applications where the number of active users are changing. In particular, an adaptive system can be established that adapts itself by designing a proper MCS not only based on the channel condition, but also based on the number of active users. Based on the demand, the active users can adjust their throughput and their code rate. For instance, if many users are present in the system, to serve them all, they cannot transmit at high data rates. However, if there are not many users and the system capacity allows, the active users can transmit at high data rates; based on their channel conditions, they can also adjust their code rates.

- This can be done more efficiently using machine learning techniques, e.g., $[143,144]$.

- A cognitive radio (CR) based SCMA system can be developed, wherein the primary users are orthogonal users, and the secondary users deploy SCMA to opportunistically transmit over the available REs. Based on their channel conditions and the number of active primary users and secondary users, MCS can be adjusted dynamically to maximize the number of accommodated users. 


\subsubsection{Joint SCMA Spreading and Constellation Mapping}

As described in the SCMA system model in Chapter 2 and pointed out in Section 3.2, the SCMA design process can be simplified by designing the user-to-RE mapping/spreading matrix first, and then considering each SCMA codeword as a product of the spreading matrix with a multidimensional constellation. However, one primary difference between SCMA and the other NOMA techniques, including LDS, is to perform the SCMA spreading and constellation mapping in one step. In other words, in SCMA systems, the SCMA spreading and the constellation mapping steps are merged together to directly map a sequence of input bits into a complex sparse codeword $[29,41]$. This requires the joint design of SCMA spreading and constellation mapping. The permutation-based SCMA (PSCMA) that is proposed in [64], and described in Section 3.4, is a potential way to design such a system.

As mentioned in Section 3.4, unlike the conventional SCMA systems, each PSCMA user is not assigned to a fixed user-to-RE mapping/spreading matrix; the position of the non-zero elements in the user-to-RE mapping matrix for each PSCMA user is based on the permutation that is a function of the transmitted bit streams, and is designed to provide a good minimum Euclidean distance between the interfering users in each RE. In other words, the codebook of each user changes with the incoming bits, and therefore MPA cannot be used as the detection algorithm. The following can be done to improve the PSCMA systems:

- Since the PSCMA codebooks in [64] are designed to maintain a good minimum Euclidean distance, based on the insights form Chapter 3, we can instead consider other KPIs to optimize the PSCMA codebooks, and obtain more gains compared with the conventional SCMA.

- The conventional widely-used MPA can be modified to support PSCMA.

\subsubsection{Joint Detection and Decoding for SCMA}

Throughout this thesis, it is assumed that the SCMA detection and the channel decoding are performed separately in a non-iterative manner. It is shown in e.g., [145] that an iterative multiuser receiver improves the SCMA system's performance. In this line, some possible research directions are as follows:

- The effect of iterative decoding and detection, i.e., BICM with iterative decoding (BICM-ID), (e.g., $[59,145,146])$ on SCMA constellations can be investigated. 
- The proposed ISD scheme in Chapter 5 can be extended to coded systems using the list SD proposed in, e.g., [105,118]. Further, since the structure of polar codes allows using SD for polar decoding, e.g., [147-149], a joint polarcoded detection and decoding SD-based algorithm can be developed for SCMA systems, which is expected to outperform the existing schemes.

\subsubsection{Grant-free SCMA}

Grant-free transmission is a method that alleviates the need for a dynamic scheduling request and the overhead inherited from a grant signaling in an uplink data transmission. In other words, in an uplink grant-free transmission, users can transmit in an "arrive-and-go" way. One of the promising applications of SCMA is for grant-free uplink scenarios [3]. A grant-free SCMA system can be developed that allows the users to blindly select their REs. In this line, the following can be investigated:

- If two users choose the same set of REs, there will be a collision. The effect of having a collision, with methods to deal with it, can be studied. Note that the likelihood of having colliding users should be reduced with the size of the system $[150,151]$.

- Since users blindly select their REs, an RE-detector at the receiver should be developed to detect the REs selected by each user.

- Efficient ways to estimate the user's channel coefficients can be demonstrated e.g., [152-155].

- A non-coherent SCMA scheme can be developed in which the receiver is able to detect the transmitted data by each user without having the knowledge of their channel coefficients. One approach to detect SCMA data without CSI at the receiver can be employing the semidefinite relaxation (SDR) technique, e.g., [156]. 


\section{List of References}

[1] Altera Innovate Asia FPGA Design Contest, "5G Algorithm Innovation Competition," 2015.

[2] Y. Yuan, Z. Yuan, G. Yu, C.-H. Hwang, P.-K. Liao, A. Li, and K. Takeda, "Non-orthogonal transmission technology in LTE evolution," IEEE Commun. Mag., vol. 54, no. 7, pp. 68-74, Jul. 2016.

[3] Y. Chen, A. Bayesteh, Y. Wu, B. Ren, S. Kang, S. Sun, Q. Xiong, C. Qian, B. Yu, Z. Ding, S. Wang, S. Han, X. Hou, H. Lin, R. Visoz, and R. Razavi, "Towards the standardization of non-orthogonal multiple access for next generation wireless networks," IEEE Commun. Mag., vol. 56, no. 3, pp. 19-27, Mar. 2018.

[4] Y. Saito, Y. Kishiyama, A. Benjebbour, T. Nakamura, A. Li, and K. Higuchi, "Non-orthogonal multiple access (NOMA) for cellular future radio access," in Proc. IEEE Vehicular Technology Conference (VTC2013-Spring), June 2013.

[5] Z. Ding, Y. Liu, J. Choi, Q. Sun, M. Elkashlan, C. L. I, and H. V. Poor, "Application of non-orthogonal multiple access in LTE and 5G networks," IEEE Commun. Mag., vol. 55, no. 2, pp. 185-191, Feb. 2017.

[6] M. Shafi, A. F. Molisch, P. J. Smith, T. Haustein, P. Zhu, P. De Silva, F. Tufvesson, A. Benjebbour, and G. Wunder, "5G: A tutorial overview of standards, trials, challenges, deployment, and practice," IEEE J. Sel. Areas Commun., vol. 35, no. 6, pp. 1201-1221, June 2017.

[7] W. Shin, M. Vaezi, B. Lee, D. J. Love, J. Lee, and H. V. Poor, "Non-orthogonal multiple access in multi-cell networks: Theory, performance, and practical challenges," IEEE Commun. Mag., vol. 55, no. 10, pp. 176-183, Oct. 2017.

[8] M. Agiwal, A. Roy, and N. Saxena, "Next generation 5G wireless networks: A comprehensive survey," IEEE Commun. Surveys Tuts., vol. 18, pp. 1617-1655, Third Quart. 2016.

[9] C. Sexton, N. J. Kaminski, J. M. Marquez-Barja, N. Marchetti, and L. A. DaSilva, "5G: Adaptable networks enabled by versatile radio access technologies," IEEE Commun. Surveys Tuts., vol. 19, pp. 688-720, Second Quart. 2017.

[10] M. Vaezi, Z. Ding, and H. V. Poor, "NOMA in code and other domains," in Multiple Access Techniques for $5 G$ Wireless Networks and Beyond, Springer, 2019. 
[11] L. Dai, B. Wang, Y. Yuan, S. Han, C. l. I, and Z. Wang, "Non-orthogonal multiple access for $5 \mathrm{G}$ : solutions, challenges, opportunities, and future research trends," IEEE Commun. Mag., vol. 53, no. 9, pp. 74-81, Sep. 2015.

[12] J. Wang, A. Jin, D. Shi, L. Wang, H. Shen, D. Wu, L. Hu, L. Gu, L. Lu, Y. Chen, J. Wang, Y. Saito, A. Benjebbour, and Y. Kishiyama, "Spectral efficiency improvement with 5G technologies: Results from field tests," IEEE J. Sel. Areas Commun., vol. 35, no. 8, pp. 1867-1875, Aug. 2017.

[13] P. Porambage, J. Okwuibe, M. Liyanage, M. Ylianttila, and T. Taleb, "Survey on multi-access edge computing for Internet of Things realization," IEEE Commun. Surveys Tuts., vol. 20, pp. 2961-2991, Fourth Quart. 2018.

[14] Y. Cai, Z. Qin, F. Cui, G. Y. Li, and J. A. McCann, "Modulation and multiple access for 5G networks," IEEE Commun. Surveys Tuts., vol. 20, pp. 629-646, First Quart. 2018.

[15] Z. Ding, X. Lei, G. K. Karagiannidis, R. Schober, J. Yuan, and V. K. Bhargava, "A survey on non-orthogonal multiple access for $5 \mathrm{G}$ networks: Research challenges and future trends," IEEE J. Sel. Areas Commun., vol. 35, no. 10, pp. 2181-2195, Oct. 2017.

[16] J. Choi, "Non-orthogonal multiple access in downlink coordinated two-point systems," IEEE Commun. Lett., vol. 18, no. 2, pp. 313-316, Feb. 2014.

[17] S. M. R. Islam, N. Avazov, O. A. Dobre, and K. S. Kwak, "Power-domain nonorthogonal multiple access (NOMA) in 5G systems: Potentials and challenges," IEEE Commun. Surveys Tuts., vol. 19, pp. 721-742, Second Quart. 2017.

[18] J. Guo, X. Zhou, S. Durrani, and H. Yanikomeroglu, "Design of non-orthogonal multiple access enhanced backscatter communication," IEEE Trans. Wireless Commun., vol. 17, no. 10, pp. 6837-6852, Oct. 2018.

[19] Z. Chen, Z. Ding, X. Dai, and R. Zhang, "An optimization perspective of the superiority of NOMA compared to conventional OMA," IEEE Trans. Signal Process., vol. 65, no. 19, pp. 5191-5202, Oct. 2017.

[20] M. Najafi, V. Jamali, P. D. Diamantoulakis, G. K. Karagiannidis, and R. Schober, "Non-orthogonal multiple access for FSO backhauling," in Proc. IEEE Wireless Communications and Networking Conference (WCNC), Apr. 2018.

[21] R. Hoshyar, F. P. Wathan, and R. Tafazolli, "Novel low-density signature for synchronous CDMA systems over AWGN channel," IEEE Trans. Signal Process., vol. 56, no. 4, pp. 1616-1626, Apr. 2008.

[22] R. Hoshyar, R. Razavi, and M. Al-Imari, "LDS-OFDM an efficient multiple access technique," in Proc. IEEE Vehicular Technology Conference (VTC2010Spring), May 2010. 
[23] M. Al-Imari, M. A. Imran, R. Tafazolli, and D. Chen, "Performance evaluation of low density spreading multiple access," in Proc. International Wireless Communications and Mobile Computing Conference (IWCMC), pp. 383-388, Aug. 2012 .

[24] M. A. Imran, M. Al-Imari, and R. Tafazolli, "Low density spreading multiple access," Journal of Information Technology and Software Engineering, vol. 2, no. 4, Sep. 2012.

[25] M. Al-Imari, P. Xiao, M. A. Imran, and R. Tafazolli, "Uplink non-orthogonal multiple access for 5G wireless networks," in Proc. International Symposium on Wireless Communications Systems (ISWCS), pp. 781-785, Aug. 2014.

[26] R. Razavi, R. Hoshyar, M. A. Imran, and Y. Wang, "Information theoretic analysis of LDS scheme," IEEE Commun. Lett., vol. 15, no. 8, pp. 798-800, Aug. 2011.

[27] A. Montanari and D. Tse, "Analysis of belief propagation for non-linear problems: The example of CDMA (or: How to prove Tanaka's formula)," in Proc. IEEE Information Theory Workshop (ITW), pp. 160-164, Mar. 2006.

[28] F. R. Kschischang, B. J. Frey, and H. A. Loeliger, "Factor graphs and the sumproduct algorithm," IEEE Trans. Inf. Theory, vol. 47, no. 2, pp. 498-519, Feb. 2001.

[29] H. Nikopour and H. Baligh, "Sparse code multiple access," in Proc. IEEE Annual International Symposium on Personal, Indoor, and Mobile Radio Communications (PIMRC), pp. 332-336, Sep. 2013.

[30] M. Taherzadeh, H. Nikopour, A. Bayesteh, and H. Baligh, "SCMA codebook design," in Proc. IEEE Vehicular Technology Conference (VTC2014-Fall), Sep. 2014.

[31] S. Chen, B. Ren, Q. Gao, S. Kang, S. Sun, and K. Niu, "Pattern division multiple access - a novel nonorthogonal multiple access for fifth-generation radio networks," IEEE Trans. Veh. Technol., vol. 66, no. 4, pp. 3185-3196, Apr. 2017.

[32] D. Fang, Y. C. Huang, Z. Ding, G. Geraci, S. L. Shieh, and H. Claussen, "Lattice partition multiple access: A new method of downlink non-orthogonal multiuser transmissions," in Proc. IEEE Global Communications Conference (Globecom), Dec. 2016.

[33] B. Wang, K. Wang, Z. Lu, T. Xie, and J. Quan, "Comparison study of nonorthogonal multiple access schemes for 5G," in Proc. IEEE International Symposium on Broadband Multimedia Systems and Broadcasting, June 2015.

[34] M. Moltafet, N. M. Yamchi, M. R. Javan, and P. Azmi, "Comparison study between PD-NOMA and SCMA," IEEE Trans. Veh. Technol., vol. 67, no. 2, pp. 1830-1834, Feb. 2018.

[35] Z. Wu, K. Lu, C. Jiang, and X. Shao, "Comprehensive study and comparison on 5G NOMA schemes," IEEE Access, vol. 6, pp. 18511-18519, Mar. 2018. 
[36] I. Parvez, A. Rahmati, I. Guvenc, A. I. Sarwat, and H. Dai, "A survey on low latency towards 5G: RAN, core network and caching solutions," IEEE Commun. Surveys Tuts., vol. 20, pp. 3098-3130, Fourth Quart. 2018.

[37] 3GPP R1-168067 ZTE, "WF on clarification of grant-free transmission for mMTC," Oct. 2016.

[38] Y. Chen, A. Bayesteh, Y. Wu, S. Han, M. Taherzadeh, D. Chen, and J. Ma, "SCMA: A promising non-orthogonal multiple access technology for 5G networks," in Proc. IEEE Vehicular Technology Conference (VTC-Fall), Sep. 2016.

[39] Evolved Universal Terrestrial Radio Access Network, "3rd Generation Partnership Project; Technical Specification Group Radio Access Network; Evolved Universal Terrestrial Radio Access Network," 2011.

[40] 3GPP TS 38.212, "NR; multiplexing and channel coding," 3rd Generation Partnership Project; Technical Specification Group Radio Access Network, Mar. 2019.

[41] A. Bayesteh, H. Nikopour, M. Taherzadeh, H. Baligh, and J. Ma, "Low complexity techniques for SCMA detection," in Proc. IEEE Globecom Workshops (GC Wkshps), Dec. 2015.

[42] D. Cai, P. Fan, X. Lei, Y. Liu, and D. Chen, "Multi-dimensional SCMA codebook design based on constellation rotation and interleaving," in Proc. IEEE Vehicular Technology Conference (VTC2016-Spring), May 2016.

[43] Y. Zhou, Q. Yu, W. Meng, and C. Li, "SCMA codebook design based on constellation rotation," in Proc. IEEE International Conference on Communications (ICC), May 2017.

[44] M. Alam and Q. Zhang, "Performance study of SCMA codebook design," in Proc. IEEE Wireless Communications and Networking Conference (WCNC), Mar. 2017.

[45] M. Alam and Q. Zhang, "Designing optimum mother constellation and codebooks for SCMA," in Proc. IEEE International Conference on Communications $(I C C)$, May 2017.

[46] K. Lai, J. Lei, L. Wen, G. Chen, W. Li, and P. Xiao, "Secure transmission with randomized constellation rotation for downlink sparse code multiple access system," IEEE Access, vol. 6, pp. 5049-5063, Feb. 2018.

[47] L. Yu, X. Lei, P. Fan, and D. Chen, "An optimized design of SCMA codebook based on star-QAM signaling constellations," in Proc. International Conference on Wireless Communications Signal Processing (WCSP), Oct. 2015.

[48] L. Yu, P. Fan, X. Lei, and P. T. Mathiopoulos, "BER analysis of SCMA systems with codebooks based on star-QAM signaling constellations," IEEE Commun. Lett., vol. 21, no. 9, pp. 1925-1928, Sep. 2017. 
[49] V. P. Klimentyev and A. B. Sergienko, "Error probability bounds for SCMA signals," in Proc. IEEE Conference of Russian Young Researchers in Electrical and Electronic Engineering (EIConRus), pp. 164-168, Feb. 2017.

[50] L. Li, Z. Ma, P. Fan, and L. Hanzo, "High dimensional codebook design for the SCMA down-link," IEEE Trans. Veh. Technol., vol. 67, no. 10, pp. 1011810122, Oct. 2018

[51] M. Taherzadeh, H. Nikopour, A. Bayesteh, and M. Baligh, "System and method for designing and using multidimensional constellations," Nov. 2016. US patent $9,509,379$.

[52] S. Zhang, K. Xiao, B. Xiao, Z. Chen, B. Xia, D. Chen, and S. Ma, "A capacitybased codebook design method for sparse code multiple access systems," in Proc. International Conference on Wireless Communications Signal Processing (WCSP), Oct. 2016.

[53] H. Yan, H. Zhao, Z. Lv, and H. Yang, "A top-down SCMA codebook design scheme based on lattice theory," in Proc. IEEE Annual International Symposium on Personal, Indoor, and Mobile Radio Communications (PIMRC), Sep. 2016 .

[54] C. Yan, G. Kang, and N. Zhang, "A dimension distance-based SCMA codebook design," IEEE Access, vol. 5, pp. 5471-5479, May 2017.

[55] T. Metkarunchit, "SCMA codebook design base on circular-QAM," in Proc. Integrated Communications, Navigation and Surveillance Conference (ICNS), Apr. 2017.

[56] J. Bao, Z. Ma, Z. Ding, G. K. Karagiannidis, and Z. Zhu, "On the design of multiuser codebooks for uplink SCMA systems," IEEE Commun. Lett., vol. 20, pp. 1920-1923, Oct. 2016.

[57] J. Bao, Z. Ma, M. A. Mahamadu, Z. Zhu, and D. Chen, "Spherical codes for SCMA codebook," in Proc. IEEE Vehicular Technology Conference (VTC2016Spring), May 2016.

[58] D. Zhai, "Adaptive codebook design and assignment for energy saving in SCMA networks," IEEE Access, vol. 5, pp. 23550-23562, Nov. 2017.

[59] J. Bao, Z. Ma, M. Xiao, T. A. Tsiftsis, and Z. Zhu, "Bit-interleaved coded SCMA with iterative multiuser detection: Multidimensional constellations design," IEEE Trans. Commun., vol. 66, no. 11, pp. 5292-5304, Nov. 2018.

[60] Q. He, B. Bai, D. Feng, H. Xu, and M. Zhu, "A nonbinary LDPC-coded SCMA system with optimized codebook design," in Proc. IEEE Vehicular Technology Conference (VTC201\%-Fall), Sep. 2017.

[61] H. Nikopour and M. Baligh, "Systems and methods for sparse code multiple access," Jan. 2016. US patent 9,240,853. 
[62] J. Peng, W. Chen, B. Bai, X. Guo, and C. Sun, "Joint optimization of constellation with mapping matrix for SCMA codebook design," IEEE Signal Process. Lett., vol. 24, no. 3, pp. 264-268, Mar. 2017.

[63] V. P. Klimentyev and A. B. Sergienko, "SCMA codebooks optimization based on genetic algorithm," in Proc. European Wireless Conference, May 2017.

[64] M. Kulhandjian and C. D'Amours, "Design of permutation-based sparse code multiple access system," in Proc. IEEE Annual International Symposium on Personal, Indoor, and Mobile Radio Communications (PIMRC), Oct. 2017.

[65] J. van de Beek and B. M. Popovic, "Multiple access with low-density signatures," in Proc. IEEE Global Telecommunications Conference (Globecom), Nov. 2009 .

[66] H. Khoshnevis, I. Marsland, and H. Yanikomeroglu, "Design of high-SNR multidimensional constellations for orthogonal transmission in a Nakagami- $m$ fading channel," IEEE Access, vol. 5, pp. 26623-26638, Dec. 2017.

[67] J. Proakis and M. Salehi, Digital Communications. 5th ed. NY, McGraw-Hill, 2008.

[68] M. Beko and R. Dinis, "Designing good multi-dimensional constellations," IEEE Commun. Lett., vol. 1, no. 3, pp. 221-224, Jun. 2012.

[69] E. Biglieri, J. Proakis, and S. Shamai, "Fading channels: information-theoretic and communications aspects," IEEE Trans. Inf. Theory, vol. 44, no. 6, pp. 2619-2692, Oct. 1998.

[70] J. Boutros, E. Viterbo, C. Rastello, and J. C. Belfiore, "Good lattice constellations for both Rayleigh fading and Gaussian channels," IEEE Trans. Inf. Theory, vol. 42, no. 2, pp. 502-518, Mar. 1996.

[71] J. Boutros and E. Viterbo, "Signal space diversity: a power- and bandwidthefficient diversity technique for the Rayleigh fading channel," IEEE Trans. Inf. Theory, vol. 44, no. 4, pp. 1453-1467, Jul. 1998.

[72] A. Chindapol and J. A. Ritcey, "Design, analysis, and performance evaluation for BICM-ID with square QAM constellations in Rayleigh fading channels," IEEE J. Sel. Areas Commun., vol. 19, no. 5, pp. 944-957, May 2001.

[73] P. R. Balogun, I. D. Marsland, R. H. Gohary, and H. Yanikomeroglu, "Polar code design for irregular multidimensional constellations," IEEE Access, vol. 5, pp. 21941-21953, Nov. 2017.

[74] C. Rachinger, R. R. Muller, and J. B. Huber, "Bit-interleaved coded modulation for phase shift keying on the hypersphere," in Proc. IEEE International Symposium on Information Theory (ISIT), pp. 496-500, June 2017.

[75] C. Stierstorfer and R. F. H. Fischer, "(Gray) mappings for bit-interleaved coded modulation," in Proc. IEEE Vehicular Technology Conference (VTC2007Spring), pp. 1703-1707, Apr. 2007. 
[76] E. Agrell and A. Alvarado, "On optimal constellations for BICM at low SNR," in Proc. Information Theory Workshop, pp. 480-484, Oct. 2009.

[77] E. Agrell and A. Alvarado, "Optimal alphabets and binary labelings for BICM at low SNR," IEEE Trans. Inf. Theory, vol. 57, no. 10, pp. 6650-6672, Oct. 2011.

[78] O. Alamri, B. Poupart, M. El-Hajjar, S. X. Ng, and L. Hanzo, "On multidimensional BICM-ID constellation labelling," in Proc. IEEE International Conference on Communications, May 2010.

[79] G. Caire, G. Taricco, and E. Biglieri, "Bit-interleaved coded modulation," IEEE Trans. Inf. Theory, vol. 44, no. 3, pp. 927-946, May 1998.

[80] Z. Bai, B. Li, M. Yang, Z. Yan, X. Zuo, and Y. Zhang, "FH-SCMA: frequencyhopping based sparse code multiple access for next generation Internet of Things," in Proc. IEEE Wireless Communications and Networking Conference (WCNC), Mar. 2017.

[81] J. Dai, K. Niu, Z. Si, and J. Lin, "Polar coded non-orthogonal multiple access," in Proc. IEEE International Symposium on Information Theory (ISIT), pp. 988-992, July 2016.

[82] A. B. Sergienko and V. P. Klimentyev, "SCMA detection with channel estimation error and resource block diversity," in Proc. International Siberian Conference on Control and Communications (SIBCON), May 2016.

[83] V. P. Klimentyev and A. B. Sergienko, "A low-complexity SCMA detector for AWGN channel based on solving overdetermined systems of linear equations," in Proc. International Symposium Problems of Redundancy in Information and Control Systems (REDUNDANCY), pp. 61-65, Sept. 2016.

[84] J. Dai, K. Niu, C. Dong, and J. Lin, "Improved message passing algorithms for sparse code multiple access," IEEE Trans. Veh. Technol., vol. 66, no. 11, pp. 9986-9999, Nov. 2017.

[85] J. Dnes and A. Keedwell, Latin Squares: New Developments in the Theory and Applications. Elsevier, 1991.

[86] Y. Xin, Z. Wang, and G. B. Giannakis, "Space-time diversity systems based on linear constellation precoding," IEEE Trans. Wireless Commun., vol. 2, no. 2, pp. 294-309, Mar. 2003.

[87] S. P. Herath, N. H. Tran, and T. Le-Ngoc, "Rotated multi-D constellations in Rayleigh fading: Mutual information improvement and pragmatic approach for near-capacity performance in high-rate regions," IEEE Trans. Commun., vol. 60 , no. 12 , pp. 3694-3704, Dec. 2012.

[88] T. C. Wong, H. M. Kwon, and A. Mukherjee, "Hybrid multi-dimensional modulation for Gaussian and fading channels," in Proc. IEEE Vehicular Technology Conference (VTC2010-Fall), Sep. 2010. 
[89] N. Sloane, "Tables of sphere packings and spherical codes," IEEE Trans. Inf. Theory, vol. 27, no. 3, pp. 327-338, May 1981.

[90] Huawei White Paper, "5G: New air interface and radio access virtualization," April 2015.

[91] Q. Xie, Z. Yang, J. Song, and L. Hanzo, "EXIT-chart-matching-aided nearcapacity coded modulation design and a BICM-ID design example for both Gaussian and Rayleigh channels," IEEE Trans. Veh. Technol., vol. 62, no. 3, pp. 1216-1227, Mar. 2013.

[92] Z. Q. Luo, W. K. Ma, A. M. C. So, Y. Ye, and S. Zhang, "Semidefinite relaxation of quadratic optimization problems," IEEE Signal Process. Mag., vol. 27, no. 3, pp. 20-34, May 2010.

[93] C. D'Amours, "Parity bit selected spreading sequences: A block coding approach to spread spectrum," IEEE Commun. Lett., vol. 9, no. 1, pp. 16-18, Jan. 2005.

[94] M. Shi, C. D'Amours, and A. Yongacoglu, "Design of spreading permutations for MIMO-CDMA based on space-time block codes," IEEE Commun. Lett., vol. 14, no. 1, pp. 36-38, Jan. 2010.

[95] S. Moussa, A. O. Dahmane, C. D’Amours, and H. Hamam, "MIMO-OFDM scheme based on permutation spreading," in Proc. International Conference on Advanced Communication Technology (ICACT), pp. 679-682, Feb. 2012.

[96] G. Romano, F. Palmieri, and P. K. Willett, "Soft iterative decoding for overloaded CDMA," in Proc. IEEE International Conference on Acoustics, Speech, and Signal Processing., vol. 3, Mar. 2005.

[97] M. Dabiri and H. Saeedi, "Dynamic SCMA codebook assignment methods: A comparative study," IEEE Commun. Lett., vol. 22, no. 2, pp. 364-367, Feb. 2018.

[98] T. S. Rappaport, Wireless Communications: Principles and Practice. Prentice Hall, 1996.

[99] H. Zarrinkoub, Understanding LTE with MATLAB: from Mathematical Modeling to Simulation and Prototyping. John Wiley \& Sons, 2014.

[100] R. Knopp and P. A. Humblet, "On coding for block fading channels," IEEE Trans. Inf. Theory, vol. 46, no. 1, pp. 189-205, Jan. 2000.

[101] H. Gavin, "The Levenberg-Marquardt method for nonlinear least squares curvefitting problems," Department of Civil and Environmental Engineering, Duke University, pp. 1-15, 2011.

[102] G. H. Golub and C. F. Van Loan, Matrix computations, vol. 3. JHU press, 2012.

[103] L. Tian, J. Zhong, M. Zhao, and L. Wen, "A suboptimal algorithm for SCMA codebook design over uplink Rayleigh fading channels," in Proc. IEEE Vehicular Technology Conference (VTC2018-Spring), Jun. 2018. 
[104] S. Lim and H. Park, "Codebook optimization for the superposition transmission of uplink SCMA systems," IEEE Trans. Veh. Technol., vol. 67, no. 10, pp. 10112-10117, Oct. 2018.

[105] F. Wei and W. Chen, "Low complexity iterative receiver design for sparse code multiple access," IEEE Trans. Commun., vol. 65, no. 2, pp. 621-634, Feb. 2017.

[106] N. H. Mahmood, H. Alves, O. A. López, M. Shehab, D. P. M. Osorio, and M. Latva-aho, "Six key enablers for machine type communication in 6G," arXiv preprint arXiv:1903.05406, Mar. 2019.

[107] J. Jalden and B. Ottersten, "On the complexity of sphere decoding in digital communications," IEEE Trans. Signal Process., vol. 53, no. 4, pp. 1474-1484, Apr. 2005.

[108] F. Wei and W. Chen, "A low complexity SCMA decoder based on list sphere decoding," in Proc. IEEE Global Communications Conference (GLOBECOM), Dec. 2016.

[109] L. Yang, X. Ma, and Y. Siu, "Low complexity MPA detector based on sphere decoding for SCMA," IEEE Commun. Lett., vol. 21, no. 8, pp. 1855-1858, Aug. 2017.

[110] G. Chen, J. Dai, K. Niu, and C. Dong, "Optimal receiver design for SCMA system," in Proc. IEEE Annual International Symposium on Personal, Indoor, and Mobile Radio Communications (PIMRC), Oct. 2017.

[111] G. Chen, J. Dai, K. Niu, and C. Dong, "Sparsity-inspired sphere decoding (SI-SD): A novel blind detection algorithm for uplink grant-free sparse code multiple access," IEEE Access, vol. 5, pp. 19983-19993, Oct. 2017.

[112] L. Li, J. Wen, X. Tang, and C. Tellambura, "Modified sphere decoding for sparse code multiple access," IEEE Commun. Lett., vol. 22, no. 8, pp. 1544-1547, Aug. 2018.

[113] Y. Han, W. Zhou, M. Zhao, and S. Zhou, "Enabling high order SCMA systems in downlink scenarios with a serial coding scheme," IEEE Access, vol. 6, pp. 33796-33809, July 2018.

[114] L. Karakchieva and P. Trifonov, "Joint list multistage decoding with sphere detection for polar coded SCMA systems," in Proc. International ITG Conference on Systems, Communications and Coding, Feb. 2019.

[115] B. Hassibi and H. Vikalo, "On the sphere-decoding algorithm I. Expected complexity," IEEE Trans. Signal Process., vol. 53, no. 8, pp. 2806-2818, Aug. 2005.

[116] M. O. Damen, H. E. Gamal, and G. Caire, "On maximum-likelihood detection and the search for the closest lattice point," IEEE Trans. Inf. Theory, vol. 49, no. 10, pp. 2389-2402, Oct. 2003.

[117] B. K. Jeong, B. Shim, and K. B. Lee, "MAP-based active user and data detection for massive machine-type communications," IEEE Trans. Veh. Technol., vol. 67, no. 9, pp. 8481-8494, Sep. 2018. 
[118] B. M. Hochwald and S. ten Brink, "Achieving near-capacity on a multipleantenna channel," IEEE Trans. Commun., vol. 51, no. 3, pp. 389-399, Mar. 2003.

[119] Z. Ma and J. Bao, "Sparse code multiple access (SCMA)," in Multiple Access Techniques for $5 G$ Wireless Networks and Beyond, pp. 369-416, Springer, 2019.

[120] K. Nikitopoulos, G. Georgis, C. Jayawardena, D. Chatzipanagiotis, and R. Tafazolli, "Massively parallel tree search for high-dimensional sphere decoders," IEEE Trans. Parallel Distrib. Syst., to appear, 10.1109/TPDS.2018.2874002.

[121] S. Han, C. Guo, W. Meng, C. Li, Y. Cui, and W. Tang, "The uplink and downlink design of MIMO-SCMA system," in Proc. International Wireless Communications and Mobile Computing Conference (IWCMC), pp. 56-60, Sept. 2016.

[122] W. Abdessamad, Y. Nasser, K. Y. Kabalan, and O. Bazzi, "On the performance evaluation of MIMO-SCMA systems," in Proc. International Congress on Ultra Modern Telecommunications and Control Systems and Workshops (ICUMT), pp. 135-140, Oct. 2016.

[123] M. Seidl, A. Schenk, C. Stierstorfer, and J. B. Huber, "Polar-coded modulation," IEEE Trans. Commun., vol. 61, no. 10, pp. 4108-4119, Oct. 2013.

[124] H. Imai and S. Hirakawa, "A new multilevel coding method using errorcorrecting codes," IEEE Trans. Inf. Theory, vol. 23, no. 3, pp. 371-377, 1977.

[125] Y. Du, B. Dong, Z. Chen, J. Fang, and L. Yang, "Shuffled multiuser detection schemes for uplink sparse code multiple access systems," IEEE Commun. Lett., vol. 20, no. 6, pp. 1231-1234, June 2016.

[126] Y. Du, B. Dong, Z. Chen, J. Fang, and X. Wang, "A fast convergence multiuser detection scheme for uplink SCMA systems," IEEE Wireless Commun. Lett., vol. 5, no. 4, pp. 388-391, Aug. 2016.

[127] Y. Du, B. Dong, Z. Chen, J. Fang, P. Gao, and Z. Liu, "Low-complexity detector in sparse code multiple access systems," IEEE Commun. Lett., vol. 20, no. 9, pp. 1812-1815, Sep. 2016.

[128] Y. Wang and L. Qiu, "Edge selection-based low complexity detection scheme for SCMA system," in Proc. IEEE Vehicular Technology Conference (VTC2016Fall), Sep. 2016.

[129] B. Tomasi, F. Gabry, V. Bioglio, I. Land, and J. C. Belfiore, "Low-complexity receiver for multi-level polar coded modulation in non-orthogonal multiple access," in Proc. IEEE Wireless Communications and Networking Conference Workshops (WCNCW), Mar. 2017.

[130] X. Meng, Y. Wu, Y. Chen, and M. Cheng, "Low complexity receiver for uplink SCMA system via expectation propagation," in Proc. IEEE Wireless Communications and Networking Conference (WCNC), Mar. 2017. 
[131] J. Dai, K. Niu, C. Dong, and J. Lin, "Scaling factor aided iterative multiuser receiver for sparse code multiple access," IEEE Commun. Lett., vol. 21, no. 8, pp. 1723-1726, Aug. 2017.

[132] C. Zhang, Y. Luo, and Y. Chen, "A low-complexity SCMA detector based on discretization," IEEE Trans. Wireless Commun., vol. 17, no. 4, pp. 2333-2345, Apr. 2018.

[133] M. Jia, L. Wang, Q. Guo, X. Gu, and W. Xiang, "A low complexity detection algorithm for fixed up-link SCMA system in mission critical scenario," IEEE Internet Things J., vol. 5, no. 5, pp. 3289-3297, 2018.

[134] O. Shental, B. M. Zaidel, and S. S. Shitz, "Low-density code-domain NOMA: Better be regular," in Proc.IEEE International Symposium on Information Theory (ISIT), pp. 2628-2632, June 2017.

[135] S. Zhang, B. Xiao, K. Xiao, Z. Chen, and B. Xia, "Design and analysis of irregular sparse code multiple access," in Proc. International Conference on Wireless Communications Signal Processing (WCSP), Oct. 2015.

[136] L. Yu, P. Fan, Z. Ma, X. Lei, and D. Chen, "An optimized design of irregular SCMA codebook based on rotated angles and EXIT chart," in Proc. IEEE Vehicular Technology Conference (VTC2016-Fall), Sept. 2016.

[137] C. Jiang and Z. Wu, "A novel uplink NOMA scheme based on low density superposition modulation," in Proc. IEEE Vehicular Technology Conference (VTC2017-Fall), Sep. 2017.

[138] M. C. Ilter, H. Yanikomeroglu, and P. A. Dmochowski, "BER upper bound expressions in coded two-transmission schemes with arbitrarily spaced signal constellations," IEEE Commun. Lett., vol. 20, no. 2, pp. 248-251, Feb. 2016.

[139] M. C. Ilter, P. A. Dmochowski, and H. Yanikomeroglu, "Revisiting error analysis in convolutionally coded systems: The irregular constellation case," IEEE Trans. Commun., vol. 66, no. 2, pp. 465-477, Feb. 2018.

[140] J. Bao, Z. Ma, M. Xiao, and Z. Zhu, "Error performance of sparse code multiple access networks with joint ML detection," in Proc. IEEE Vehicular Technology Conference (VTC Spring), May 2016.

[141] J. Bao, Z. Ma, G. K. Karagiannidis, M. Xiao, and Z. Zhu, "Joint multiuser detection of multidimensional constellations over fading channels," IEEE Trans. Commun., vol. 65, no. 1, pp. 161-172, Jan. 2017.

[142] D. Cai, P. Fan, and P. T. Mathiopoulos, "A tight lower bound for the symbol error performance of the uplink sparse code multiple access," IEEE Wireless Commun. Lett., vol. 6, no. 2, pp. 190-193, Apr. 2017.

[143] T. O'Shea and J. Hoydis, "An introduction to deep learning for the physical layer," IEEE Trans. on Cogn. Commun. Netw., vol. 3, no. 4, pp. 563-575, Dec. 2017. 
[144] M. Kim, N. Kim, W. Lee, and D. Cho, "Deep learning-aided SCMA," IEEE Commun. Lett., vol. 22, no. 4, pp. 720-723, Apr. 2018.

[145] Y. Wu, S. Zhang, and Y. Chen, "Iterative multiuser receiver in sparse code multiple access systems," in Proc. IEEE International Conference on Communications (ICC), pp. 2918-2923, June 2015.

[146] J. Bao, Z. Ma, M. Xiao, T. A. Tsiftsis, and Z. Zhu, "Performance analysis of uplink sparse code multiple access with iterative multiuser receiver," in Proc. IEEE International Conference on Communications (ICC), May 2017.

[147] S. A. Hashemi, C. Condo, and W. J. Gross, "List sphere decoding of polar codes," in Proc. Asilomar Conference on Signals, Systems and Computers, pp. 1346-1350, Nov. 2015.

[148] J. Guo and A. G. i Fabregas, "Efficient sphere decoding of polar codes," in Proc. IEEE International Symposium on Information Theory (ISIT), pp. 236240, June 2015.

[149] K. Niu, K. Chen, and J. Lin, "Low-complexity sphere decoding of polar codes based on optimum path metric," IEEE Commun. Lett., vol. 18, no. 2, pp. 332335, Feb. 2014.

[150] A. Bayesteh, E. Yi, H. Nikopour, and H. Baligh, "Blind detection of SCMA for uplink grant-free multiple-access," in Proc. International Symposium on Wireless Communications Systems (ISWCS), pp. 853-857, Aug. 2014.

[151] K. Au, L. Zhang, H. Nikopour, E. Yi, A. Bayesteh, U. Vilaipornsawai, J. Ma, and P. Zhu, "Uplink contention based SCMA for 5G radio access," in Proc. IEEE Globecom Workshops (GC Wkshps), pp. 900-905, Dec. 2014.

[152] F. Wei, W. Chen, Y. Wu, J. Ma, and T. A. Tsiftsis, "Message-passing receiver design for joint channel estimation and data decoding in uplink grant-free SCMA systems," IEEE Trans. Wireless Commun., to appear, 10.1109/TWC.2018.2878571.

[153] J. Heo, I. Jung, T. Kim, H. Kim, and D. Hong, "Channel estimation for uplink SCMA systems with reduced training blocks," in Proc. IEEE Vehicular Technology Conference (VTC2018-Fall), June 2018.

[154] C. Yang, W. Xu, Z. Zhang, X. You, and C. Zhang, "A channel-blind detection for SCMA based on image processing techniques," in Proc. IEEE International Symposium on Circuits and Systems (ISCAS), May 2018.

[155] J. Liu, G. Wu, S. Li, and O. Tirkkonen, "Blind detection of uplink grant-free SCMA with unknown user sparsity," in Proc. IEEE International Conference on Communications (ICC), May 2017.

[156] W.-K. Ma, B.-N. Vo, T. N. Davidson, and P.-C. Ching, "Blind ML detection of orthogonal space-time block codes: efficient high-performance implementations," IEEE Trans. Signal Process., vol. 54, no. 2, pp. 738-751, Feb. 2006. 


\section{Appendix A}

\section{SCMA Mapping and Spreading}

To illustrate the SCMA mapping, for a scenario with $K=6, N=4, d_{v}=2$, and $M=4$, the 4-point 2-dimensional complex constellations in $[1]^{1}$ are provided in Table A1. Let take the first user as an example. The first element of $\mathcal{X}_{1}$ is $\mathbf{x}_{1,1}=$ $(-0.182-0.132 i,+0.7851)^{\mathrm{T}}$, the second element $\mathrm{x}_{1,2}=(-0.635-0.462 i,-0.224)^{\mathrm{T}}$, the third element $\mathbf{x}_{1,3}=(+0.635+0.462 i,+0.224)^{\mathrm{T}}$, and the fourth element of $\mathbf{X}_{1}$ is $\mathbf{x}_{1,4}=(+0.182+0.132 i,-0.785)^{\mathrm{T}}$. In that case, if $c_{1, i}=00$ then $\tilde{\mathbf{x}}_{1, i}=\mathbf{x}_{1,1}$, if $c_{1, i}=01$ then $\tilde{\mathbf{x}}_{1, i}=\mathbf{x}_{1,2}$, if $c_{1, i}=10$ then $\tilde{\mathbf{x}}_{1, i}=\mathbf{x}_{1,3}$, and if $c_{1, i}=11$ then $\tilde{\mathbf{x}}_{1, i}=\mathbf{x}_{1,4}$.

To illustrate the SCMA spreading, for the binary user-to-RE spreading matrix in (2.2), the codebooks corresponding to the 4-point 2-dimensional complex constellations in Table A1 are provided in Table A2. Each element $\mathbf{v}_{k, m} \in \mathcal{V}_{k}$ is given by $\mathbf{v}_{k, m}=\mathbf{S}_{k} \mathbf{x}_{k, m}$. In other words, each 2-dimensional element of $\mathcal{X}_{1}$ is spread over its corresponding 4-dimensional element of $\mathcal{V}_{1}$. From (2.2), since the first user is assigned to the second and the fourth REs, it transmits the first complex dimension of its 2-dimensional complex constellation over the second RE, and the second complex dimension of its constellation over the fourth RE. In other words, no data are transmitted by the first user on the first and the third REs. Thus, the first and third entries in each element of $\mathcal{V}_{1}$ are zeros.

\footnotetext{
${ }^{1}$ As we mentioned in Chapter 3.4, the constellation proposed in [1] is based on the T4QAM mother constellation that is then rotated according to 2-dimensional user-specific rotations in order to obtain user-specific constellations. As elaborated in Appendix B, the effect of those types of user-specific rotations becomes questionable in uplink scenarios. As such, the error performance of the constellations in [1] is the same as the case when the T4QAM constellation is used for all users with no such 2-dimensional user-specific rotations.
} 
Table A1: An example of a 4-point multidimensional constellation for an SCMA system with $K=6, N=4, d_{v}=2[1]$.

$$
\begin{aligned}
& \mathcal{X}_{1}=\left\{\left[\begin{array}{c}
-0.182-0.132 i \\
+0.785
\end{array}\right],\left[\begin{array}{c}
-0.635-0.462 i \\
-0.224
\end{array}\right],\left[\begin{array}{c}
+0.635+0.462 i \\
+0.224
\end{array}\right],\left[\begin{array}{c}
+0.182+0.132 i \\
-0.785
\end{array}\right]\right\} \\
& \mathcal{X}_{2}=\left\{\left[\begin{array}{c}
+0.785 \\
-0.182-0.132 i
\end{array}\right],\left[\begin{array}{c}
-0.224 \\
-0.635-0.462 i
\end{array}\right],\left[\begin{array}{c}
+0.224 \\
+0.635+0.462 i
\end{array}\right],\left[\begin{array}{c}
-0.785 \\
+0.182+0.132 i
\end{array}\right]\right\} \\
& \mathcal{X}_{3}=\left\{\left[\begin{array}{l}
-0.635+0.462 i \\
+0.139-0.176 i
\end{array}\right],\left[\begin{array}{c}
+0.182-0.132 i \\
+0.487-0.616 i
\end{array}\right],\left[\begin{array}{c}
-0.182+0.132 i \\
-0.487+0.616 i
\end{array}\right],\left[\begin{array}{c}
+0.635-0.462 i \\
-0.139+0.176 i
\end{array}\right]\right\} \\
& \mathcal{X}_{4}=\left\{\left[\begin{array}{c}
+0.785 \\
-0.006-0.224 i
\end{array}\right],\left[\begin{array}{c}
-0.224 \\
-0.019-0.785 i
\end{array}\right],\left[\begin{array}{c}
+0.224 \\
+0.019+0.785 i
\end{array}\right],\left[\begin{array}{c}
-0.785 \\
+0.006+0.224 i
\end{array}\right]\right\} \\
& \mathcal{X}_{5}=\left\{\left[\begin{array}{l}
-0.006-0.224 i \\
-0.635+0.462 i
\end{array}\right],\left[\begin{array}{c}
-0.019-0.785 i \\
+0.182-0.132 i
\end{array}\right],\left[\begin{array}{c}
+0.019+0.785 i \\
-0.182+0.132 i
\end{array}\right],\left[\begin{array}{l}
+0.006+0.224 i \\
+0.635-0.462 i
\end{array}\right]\right\} \\
& \mathcal{X}_{6}=\left\{\left[\begin{array}{c}
+0.785 \\
+0.139-0.176 i
\end{array}\right],\left[\begin{array}{c}
-0.224 \\
+0.487-0.616 i
\end{array}\right],\left[\begin{array}{c}
-0.487+0.616 i \\
+0.224 \\
-0.139+0.176 i
\end{array}\right]\right\}
\end{aligned}
$$


Table A2: The SCMA codebook with the multidimensional constellation example in Table A1 and the user-to-RE spreading matrix in (2.2).

$$
\begin{aligned}
& \mathcal{V}_{1}=\left\{\left[\begin{array}{c}
-0.182-0.132 i \\
0 \\
+0.785 \\
0
\end{array}\right],\left[\begin{array}{c}
-0.635-0.462 i \\
0 \\
-0.224 \\
0
\end{array}\right],\left[\begin{array}{c}
+0.635+0.462 i \\
0 \\
+0.224 \\
0
\end{array}\right],\left[\begin{array}{c}
+0.182+0.132 i \\
0 \\
-0.785 \\
0
\end{array}\right]\right\} \\
& \mathcal{V}_{2}=\left\{\left[\begin{array}{c}
0 \\
+0.785 \\
0 \\
-0.182-0.132 i
\end{array}\right],\left[\begin{array}{c}
0 \\
-0.224 \\
0 \\
-0.635-0.462 i
\end{array}\right],\left[\begin{array}{c}
0 \\
+0.224 \\
0 \\
+0.635+0.462 i
\end{array}\right],\left[\begin{array}{c}
0 \\
-0.785 \\
0 \\
+0.182+0.132 i
\end{array}\right]\right\} \\
& \mathcal{V}_{3}=\left\{\left[\begin{array}{c}
0 \\
0 \\
-0.635+0.462 i \\
+0.139-0.176 i
\end{array}\right],\left[\begin{array}{c}
0 \\
0 \\
+0.182-0.132 i \\
+0.487-0.616 i
\end{array}\right],\left[\begin{array}{c}
0 \\
0 \\
-0.182+0.132 i \\
-0.487+0.616 i
\end{array}\right],\left[\begin{array}{c}
0 \\
0 \\
+0.635-0.462 i \\
-0.139+0.176 i
\end{array}\right]\right\} \\
& \mathcal{V}_{4}=\left\{\left[\begin{array}{c}
+0.785 \\
-0.006-0.224 i \\
0 \\
0
\end{array}\right],\left[\begin{array}{c}
-0.224 \\
-0.019-0.785 i \\
0 \\
0
\end{array}\right],\left[\begin{array}{c}
+0.224 \\
+0.019+0.785 i \\
0 \\
0
\end{array}\right],\left[\begin{array}{c}
-0.785 \\
+0.006+0.224 i \\
0 \\
0
\end{array}\right]\right\} \\
& \mathcal{V}_{5}=\left\{\left[\begin{array}{c}
0 \\
-0.006-0.224 i \\
-0.635+0.462 i \\
0
\end{array}\right],\left[\begin{array}{c}
0 \\
-0.019-0.785 i \\
+0.182-0.132 i \\
0
\end{array}\right],\left[\begin{array}{c}
0 \\
+0.019+0.785 i \\
-0.182+0.132 i \\
0
\end{array}\right],\left[\begin{array}{c}
0 \\
+0.006+0.224 i \\
+0.635-0.462 i \\
0
\end{array}\right]\right\} \\
& \mathcal{V}_{6}=\left\{\left[\begin{array}{c}
+0.785 \\
0 \\
0 \\
+0.139-0.176 i
\end{array}\right],\left[\begin{array}{c}
-0.224 \\
0 \\
0 \\
+0.487-0.616 i
\end{array}\right],\left[\begin{array}{c}
+0.224 \\
0 \\
0 \\
-0.487+0.616 i
\end{array}\right],\left[\begin{array}{c}
-0.785 \\
0 \\
0 \\
-0.139+0.176 i
\end{array}\right]\right\}
\end{aligned}
$$




\section{Appendix B}

\section{Effects of User-specific Rotations}

The optimization of 2-dimensional user-specific rotations, on each RE assigned to each user independently from other REs assigned to that user, is of importance only in downlink scenarios [30], because in uplink SCMA systems the users experience

different fading channels, so the benefits of user-specific rotations are neglected. For illustrative purposes, consider a downlink and an uplink scenario with two users depicted in Fig. B.1(a) and Fig. B.1(b). Let $x_{1}$ and $x_{2}$ denote the symbols corresponding to User 1 and User 2 , and $h_{1}$ and $h_{2}$ represent the fading channel coefficients of User 1 and User 2, respectively. As we see in Fig. B.1(a), in a downlink scenario each user receives a combination of both symbols that are rotated and scaled by the same channel coefficient, i.e., $h_{1}\left(x_{1}+x_{2}\right)$ at User 1 , or $h_{2}\left(x_{1}+x_{2}\right)$ at User 2. Careful selection of the user-specific rotations in $x_{1}$ and $x_{2}$ facilitates recovering the symbol of each user from the sum, $x_{1}+x_{2}$. In contrast, as we see in Fig. B.1(b), in an uplink scenario when both users experience different fading channels, each symbol corresponding to each user is rotated and scaled by a different random value, i.e., $x_{1} h_{1}$ and $x_{2} h_{2}$. As such, any benefit of a 2-dimensional rotation on each symbol prior to transmission will be negated by the random rotation caused by random fading channel coefficients. Restated, a 2-dimensional rotation on each RE has no impact in an uplink scenario. 


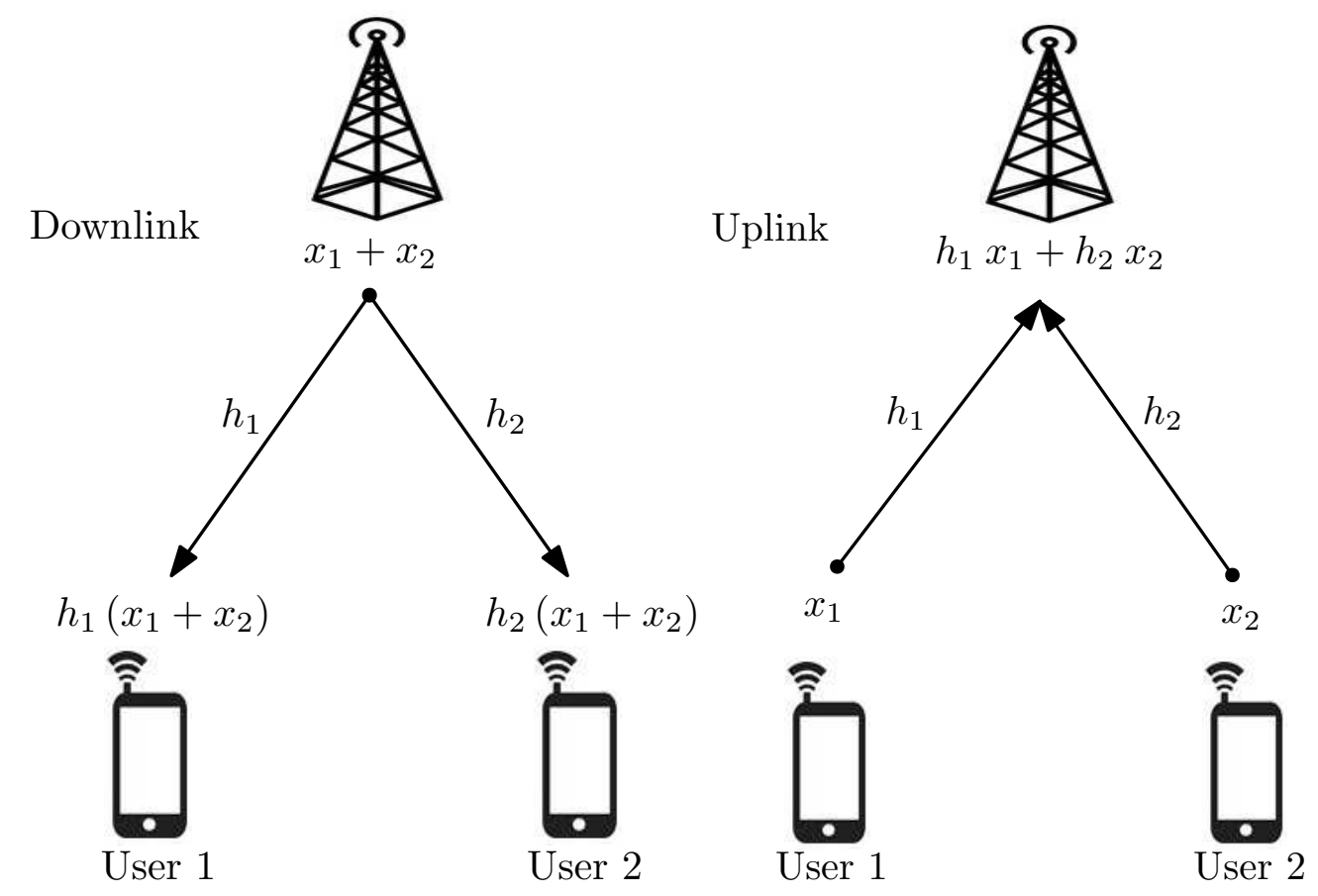

(a)

(b)

Figure B1: The illustration of the importance of user-specific rotations in (a) a downlink, and (b) an uplink scenario. 


\section{Appendix $\mathrm{C}$}

\section{Message Passing Algorithm and Bit-LLR Calculation for BICM}

From the $N \times K$ user-to-RE binary indicator matrix, F, of an SCMA system introduced in Chapter 2, the structure of an SCMA code can be represented by a bipartite graph $\mathcal{G}(K, N)$ that contains $K$ variable nodes and $N$ check nodes. The variable nodes and check nodes represent $K$ users and $N$ resources, respectively. Fig. C1 shows an example of $\mathcal{G}(K, N)$ with $K=6, N=4$, for the user-to-RE indicator matrix given in (2.3). Let $f_{n, k}$ denote the element located in the $n$th row and $k$ th column of $\mathbf{F}$, $n \in\{1, \ldots, N\}, k \in\{1, \ldots, K\}$. There is an edge between the variable node $k$ and the check node $n$ if and only if $f_{n, k}=1$. The set of check nodes connected to variable node $k$ is specified by the positions of the 1's in the $k$ th column of the indicator matrix and is represented by $\mathcal{D}_{k}=\left\{n \mid f_{n, k}=1\right\}$. In a similar vein, the set of variable nodes connected to check node $n$ is identified by the positions of the 1's in the $n$th row of $\mathbf{F}$ and is denoted by $C_{n}=\left\{k \mid f_{n, k}=1\right\}$.

Let $\mathbf{v}_{k, m}$ denote each $N$-dimensional sparse complex codeword of the $N$-dimensional sparse codebook for user $k, \mathcal{V}_{k}=\left\{\mathbf{v}_{k, m} \mid m=1, \ldots, M\right\}$ of size $M$. We assume that all codewords are selected with the same probabilities. Let $\mu_{k \rightarrow n}^{(t, i)}\left(\mathbf{v}_{k, m}\right)$ and $\mu_{n \rightarrow k}^{(t, i)}\left(\mathbf{v}_{k, m}\right)$ represent the extrinsic messages passed from user node $k$ to resource node $n$, and from resource node $n$ to user node $k$, at the $t$ th iteration, $t \in\left\{1, \ldots, N_{\text {it }}\right\}$ and at the $i$ th use of the channel, respectively. The probability that $\tilde{\mathbf{v}}_{k, i}=\mathbf{v}_{k, m}$ at the $t$ th iteration is

$$
\mu_{k \rightarrow n}^{(t, i)}\left(\mathbf{v}_{k, m}\right)=\prod_{\bar{n} \in \mathcal{D}_{k} \backslash n} \mu_{\bar{n} \rightarrow k}^{(t, i)}\left(\mathbf{v}_{k, m}\right)
$$




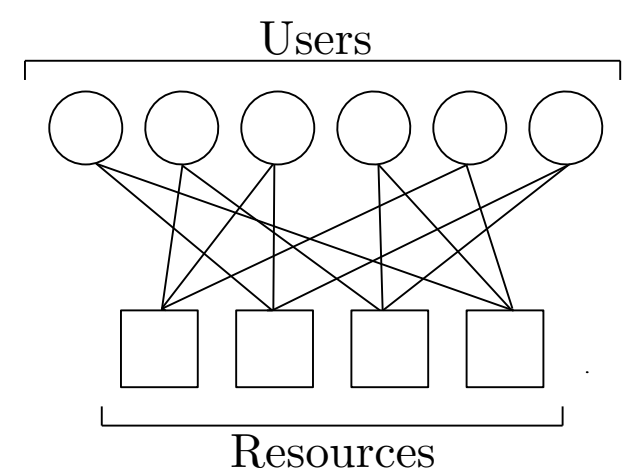

Figure C1: An example of the factor graph for $K=4$ users and $N=6$ resources.

Each element of the message conveyed from resource node $n$ to user node $k$ is

$$
\mu_{n \rightarrow k}^{(t, i)}\left(\mathbf{v}_{k, m}\right)=\sum_{\tilde{\mathbf{V}}_{i} \in \mathcal{V} \mid \bar{k} \in C_{n} \backslash k} f\left(y_{n, i} \mid \tilde{\mathbf{V}}_{i}\right) \prod_{\bar{k} \in C_{n} \backslash k} \mu_{\bar{k} \rightarrow n}^{(t, i)}\left(\mathbf{v}_{\bar{k}, m}\right)
$$

where $y_{n, i}$ is the $n$th element of received vector $\mathbf{y}_{i}$. The likelihood function is given by

$$
f\left(y_{n, i} \mid \tilde{\mathbf{V}}_{i}\right)=\frac{1}{\pi N_{0}} \exp \left\{-\frac{1}{N_{0}}\left|y_{n, i}-\sum_{k=1}^{K} h_{n, k, i} \tilde{v}_{n, k, i}\right|^{2}\right\},
$$

where $\mathcal{V}=\mathcal{V}_{1} \times \mathcal{V}_{2} \times \cdots \times \mathcal{V}_{K}$, and $\tilde{v}_{n, k, i}$ denotes each element of $\tilde{\mathbf{v}}_{k, i}$, and $\tilde{\mathbf{v}}_{k, i}$ is an $N$ dimensional sparse complex codeword, selected from $\mathcal{V}_{k}$. The a posteriori probability distribution at each variable node is then

$$
\mu_{k}^{(i)}\left(\mathbf{v}_{k, m}\right)=\beta_{k} \prod_{n \in \mathcal{D}_{k}} \mu_{n \rightarrow k}^{\left(N_{\mathrm{it}, i}\right)}\left(\mathbf{v}_{k, m}\right)
$$

where $\beta_{k}$ is chosen such that $\sum_{m=1}^{M} \mu_{k}^{(i)}\left(\mathbf{v}_{k, m}\right)=1$.

In BICM, the message words for each user of length $K_{c}$ are encoded using a single encoder with a rate of $R$ to produce codewords of length $N_{c}$. The codewords are then partitioned into $L_{M}$-tuples to be mapped to a complex codeword for each user, $\tilde{\mathbf{v}}_{k}$.

At the receiver, the output symbol probabilities of the MPA i.e., $\mu_{k}^{(i)}\left(\mathbf{v}_{k, m}\right)$ given by (C4), are passed to the channel decoder. Let $\mathbf{c}_{k, i}=\left[c_{1, k, i}, \ldots, c_{L_{M}, k, i}\right], c_{l, k, i} \in\{0,1\}$, $l \in\left\{1, \ldots, L_{M}\right\}, L_{M}=\log _{2} M$, denote the output symbol of length $L_{M}$ for user $k$ at the $i$ th instant of the channel. The bit-LLR for the $l$ th bit of the $k$ th user is calculated 
as

$$
\begin{aligned}
\lambda_{l, k, i} & =\log \frac{\operatorname{Pr}\left\{c_{l, k, i}=0 \mid \mathbf{y}_{i}\right\}}{\operatorname{Pr}\left\{c_{l, k, i}=1 \mid \mathbf{y}_{i}\right\}} \\
& =\log \frac{\sum_{\mathbf{v}_{k, m} \in \mathcal{V}_{l, k}^{0}} \mu_{k}^{(i)}\left(\mathbf{v}_{k, m}\right)}{\sum_{\mathbf{v}_{k, m} \in \mathcal{V}_{l, k}^{1}} \mu_{k}^{(i)}\left(\mathbf{v}_{k, m}\right)},
\end{aligned}
$$

where $\mathcal{V}_{l, k}^{0}$ and $\mathcal{V}_{l, k}^{0}$ are subsets of $\mathcal{V}_{k}$ corresponding to the $l$ th bit of the $k$ th user, $c_{l, k, i}=0$ and $c_{l, k, i}=1$, respectively.

In practical implementations, the exponential operations in $(\mathrm{C} 2)$ causes large dynamic ranges, which may result in a high storage burden [119]. As such, MPA in the logarithmic domain (Log-MPA) is used instead.

In Log-MPA, the messages passed from user node $k$ to resource node $n$, and from resource node $n$ to user node $k$ at the $t$ th iteration, can be expressed as

$$
\begin{gathered}
\mu_{k \rightarrow n}^{(t, i)}\left(\mathbf{v}_{k, m}\right)=\sum_{\bar{n} \in \mathcal{D}_{k} \backslash n} \mu_{\bar{n} \rightarrow k}^{(t, i)}\left(\mathbf{v}_{k, m}\right) . \\
\mu_{n \rightarrow k}^{(t, i)}\left(\mathbf{v}_{k, m}\right)=\max _{\tilde{\mathbf{V}}_{i} \in \mathcal{V} \mid \bar{k} \in C_{n} \backslash k}^{*}\left\{\log f\left(y_{n, i} \mid \tilde{\mathbf{V}}_{i}\right)+\sum_{\bar{k} \in C_{n} \backslash k} \mu_{\bar{k} \rightarrow n}^{(t, i)}\left(\mathbf{v}_{\bar{k}, m}\right)\right\},
\end{gathered}
$$

where the max is suggested in [118] as a numerically stable operation, and is defined as

$$
\begin{aligned}
\max ^{*}\left(a_{1}, a_{2}\right) & =\log \left(\exp \left(a_{1}\right)+\exp \left(a_{2}\right)\right) \\
& =\max \left(a_{1}, a_{2}\right)+\log \left(1+\exp \left(-\left|a_{1}-a_{2}\right|\right)\right) .
\end{aligned}
$$

The a posteriori probability distribution at each variable node is

$$
\mu_{k}^{(i)}\left(\mathbf{v}_{k, m}\right)=\log \beta_{k}+\sum_{n \in \mathcal{D}_{k}} \mu_{n \rightarrow k}^{\left(N_{\mathrm{it}, i}\right)}\left(\mathbf{v}_{k, m}\right)
$$

The bit-LLR for the $l$ th bit of the $k$ th user is then calculated as

$$
\lambda_{l, k, i}=\max _{\mathbf{v}_{k, m} \in \mathcal{V}_{l, k}^{0}}^{*} \mu_{k}^{(i)}\left(\mathbf{v}_{k, m}\right)-\max _{\mathbf{v}_{k, m} \in \mathcal{V}_{l, k}^{1}}^{*} \mu_{k}^{(i)}\left(\mathbf{v}_{k, m}\right)
$$




\section{Appendix D}

\section{Uplink SCMA Multidimensional Constellation with LDPC Codes}

\section{C1 Introduction}

As discussed in Section 3.3, depending on the channel scenario, the KPIs of $M$-point $d_{v}$-dimensional complex constellations are the minimum Euclidean distance, $d_{E, \min }$, the Euclidean kissing number, $\tau_{E}$, the minimum product distance, $d_{P \text {, min }}$, the product kissing number, $\tau_{P}$, the modulation diversity order, $L$, the number of distinct points, $N_{d}$, and the bit-labeling. We provide the KPIs of the 4-point constellations under study in Table 3.2. The objective is to show, through simulations, whether the same KPIs as in the LTE turbo coded case as in Chapter 3 should be considered in designing MdCs when the 5G-compliant LDPC code is used.

Since one of the attractive use-cases of SCMA systems is to accommodate many low-throughput users, e.g., IoT applications, and not increasing the individual user's throughput, we compare the FER performance of the MdCs under study for a constellation size of $M=4$, although addition simulation results confirm similar findings for $M=16$. The comparisons are made between the high-rate and the low-rate LTE turbo provided in Chapter 3 and 5G-compliant LDPC codes over the channel scenarios in Section 2.1. Both high-rate and low-rate 5G-compliant LDPC codes are according to the specifications provided in [40]. We use $K=6, N=4$, and $d_{v}=2$, a single antenna at all transmitters, and at the receiver, 3 MPA iterations for SCMA detection, and a maximum of 25 iterations for LDPC decoding. Furthermore, for both high-rate LTE turbo and 5G-compliant LDPC codes, the code rate is set to $R=4 / 5$, and the codeword length to $N_{c}=120$. For both low-rate LTE turbo and 
5G-compliant LDPC codes, the code rate is set to $R=1 / 3$, the codeword length to $N_{c}=2028$ for the LTE turbo code, and $N_{c}=2016$ for the $5 \mathrm{G}$-compliant LDPC code.
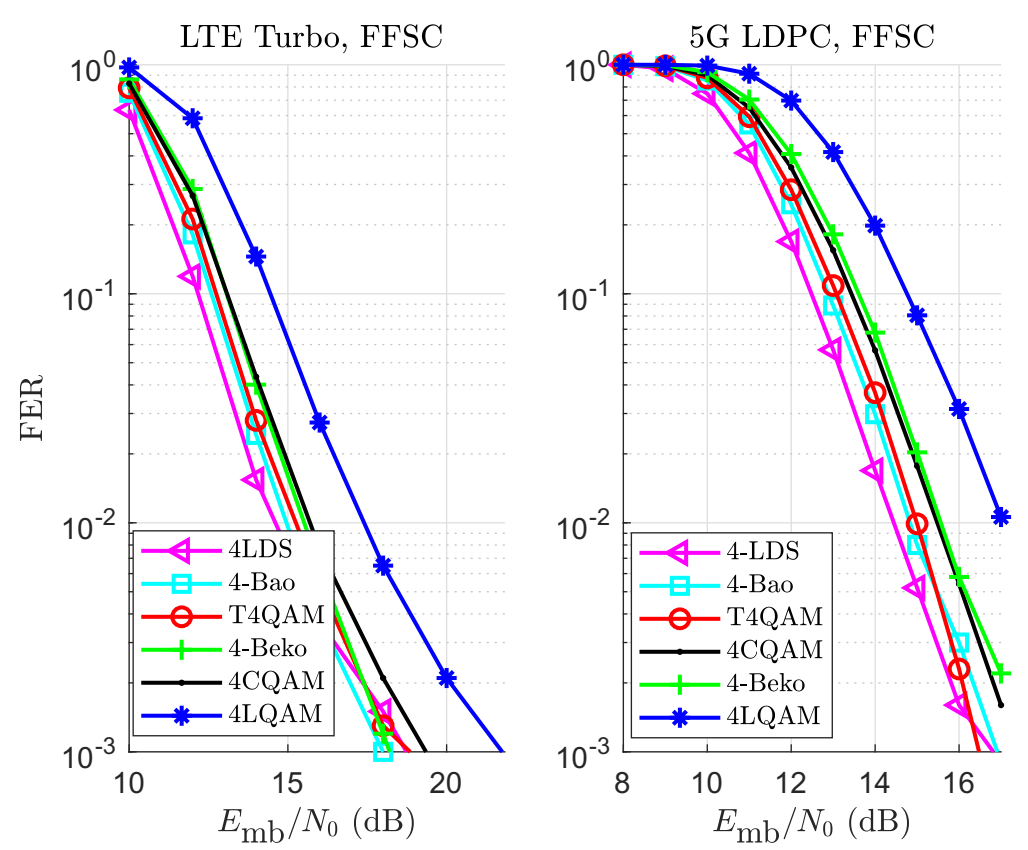

Figure C1: FER performance of LTE turbo-coded and 5G-compliant LDPC-coded systems with $R=4 / 5$ over FFSC (Case 3 ).

\section{C2 High-Rate Scenarios}

In Fig. C1, we compare the FER performance of the 4-point MdCs with the high-rate LTE turbo and 5G-compliant LDPC codes under FFSC (Case 3). As pointed out in Section 3.3, $d_{E \text {, min }}, \tau_{E}, N_{d}$, and bit-labeling are the KPIs for this scenario. From Fig. C1, we note that different MdCs comparatively perform similarly when LTE turbo code or 5G-compliant LDPC code are employed. For instance, we see that 4LDS is the best candidate when either of those coding schemes are used ${ }^{1}$. Also, from

\footnotetext{
${ }^{1}$ The detailed justification of the behaviour of MdCs over different conditions is provided in Chapter 3.
} 

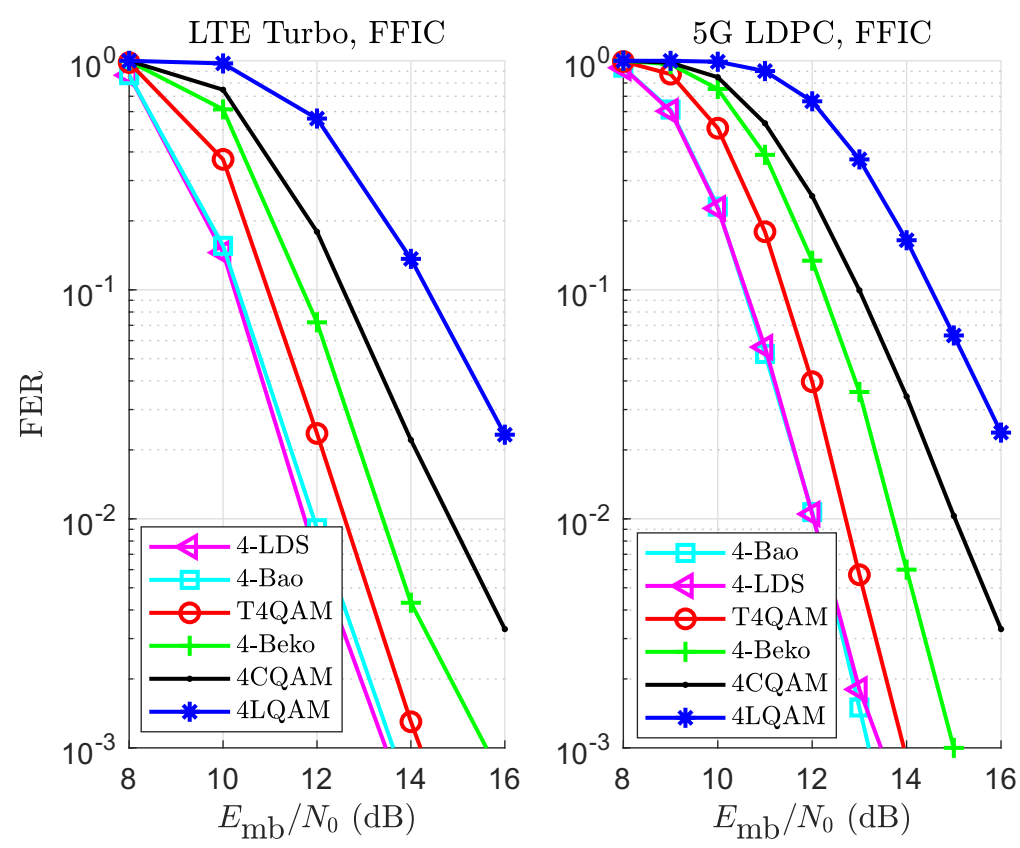

Figure C2: FER performance of LTE turbo-coded and 5G-compliant LDPC-coded systems with $R=4 / 5$ over FFIC (Case 4 ).
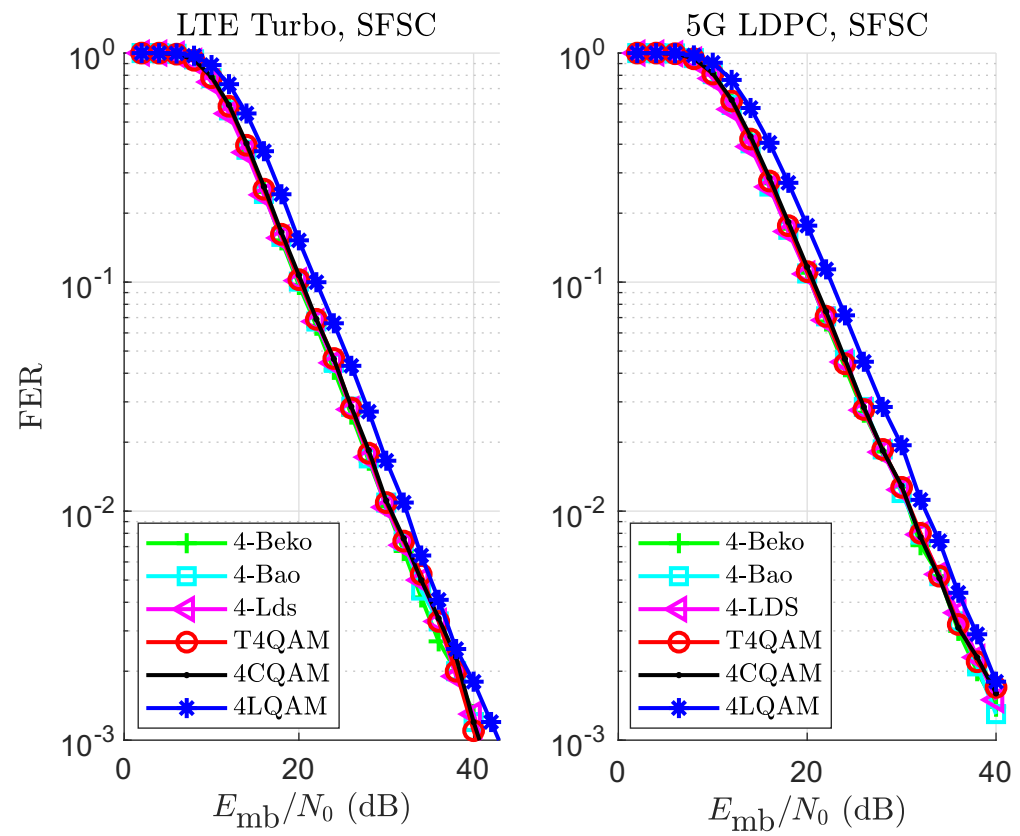

Figure C3: FER performance of LTE turbo-coded and 5G-compliant LDPC-coded systems with $R=4 / 5$ over SFSC (Case 5 ). 

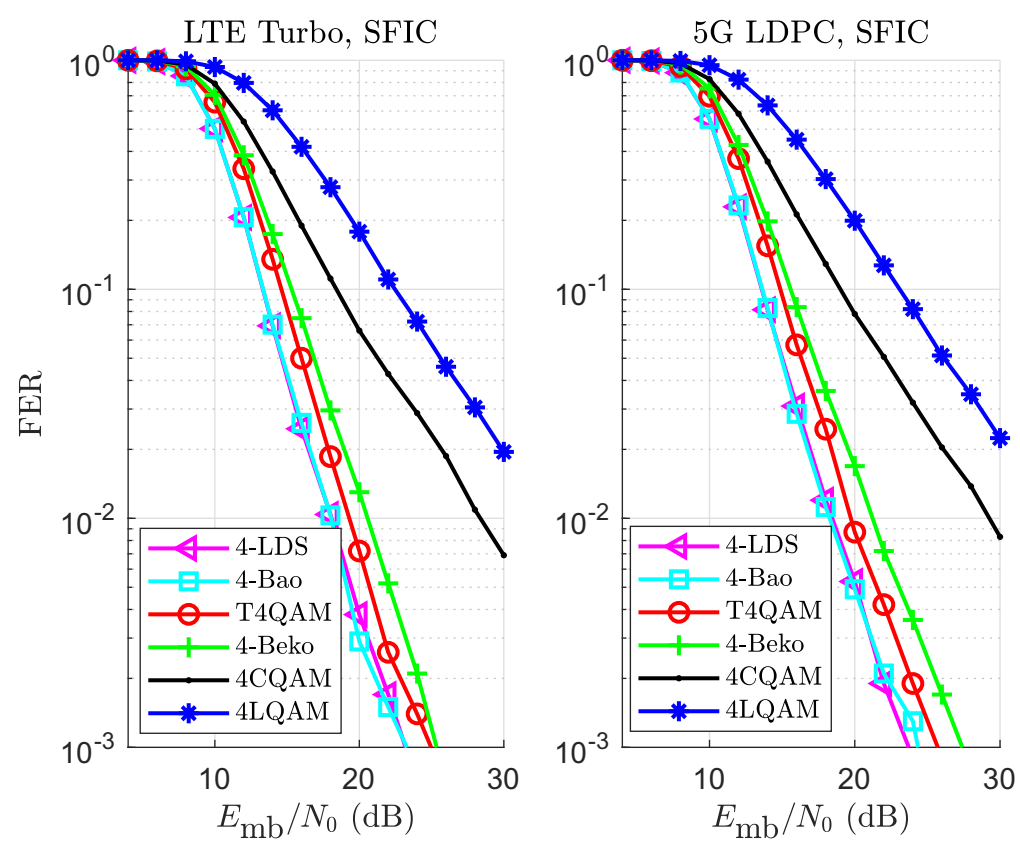

Figure C4: FER performance of LTE turbo-coded and 5G-compliant LDPC-coded systems with $R=4 / 5$ over SFIC (Case 6 ).

Table 3.2, despite the fact 4 -Beko has the highest $d_{E \text {,min }}$, it experiences performance degradation due to its bit-labeling.

In Fig. C2, we depict the FER performance of the systems described above over FFIC (Case 4). As we mentioned in Section 3.3, $N_{d}, L, d_{p \text {, min }}, \tau_{p}$, bit-labeling, and the SNR operating region, are the KPIs for this scenario. As we can see from Fig. C2, the trends of MdCs with LTE turbo code are fairly consistent with 5G-compliant LDPC code. For example, 4-LDS and 4-Bao outperform the others as listed in Table 3.2 , they have the highest combination of $L, N_{d}, d_{P \text {, min }}$ and $\tau_{P}$, compared with other MdCs.

In Fig. C3 and Fig. C4, we depict the performance of the MdCs over SFSC (Case 5) and SFIC (Case 6), respectively. Similar to FFSC, $d_{E, \min }, \tau_{E}, N_{d}$, and bit-labeling are the KPIs for SFSC. Also, similar to FFIC, $L, d_{P, \min }, \tau_{P}, N_{d}$, and bit-labeling are the KPIs for SFIC. We note from Fig. C3 and Fig. C4 that trends of the MdCs are fairly consistent when either LTE turbo or 5G-compliant LDPC codes are employed. 


\section{C3 Low-Rate Scenarios}

In Fig. C5 and Fig. C6, we compare the FER performance of the 4-point MdCs with the low-rate LTE turbo and 5G-compliant LDPC codes over FFSC and FFIC, respectively. We observe from Fig. C5-C6 that different MdCs behave similarly with both low-rate LTE turbo and 5G-compliant LDPC codes.

In Fig. C7, we evaluate the performance of the systems described above over SFSC. It is clear that the trends of MdCs are similar when either LTE turbo or $5 \mathrm{G}$-compliant LDPC codes are used. It is notable that in the presence of low-rate codes, the impact of $N_{d}$ is of less importance compared with the high-rate codes (Section 3.5.3). Moreover, from Table 3.2, all the Gray-labeled MdCs with the same $d_{E, \text { min }}$ and $\tau_{E}$ perform similarly.

Similar results, with one notable exception, occur in the SFIC case, as shown in Fig. C8. The exception is the performance of 4LQAM which is significantly worse than predicted by the KPIs when the 5G-compliant LDPC code is used.
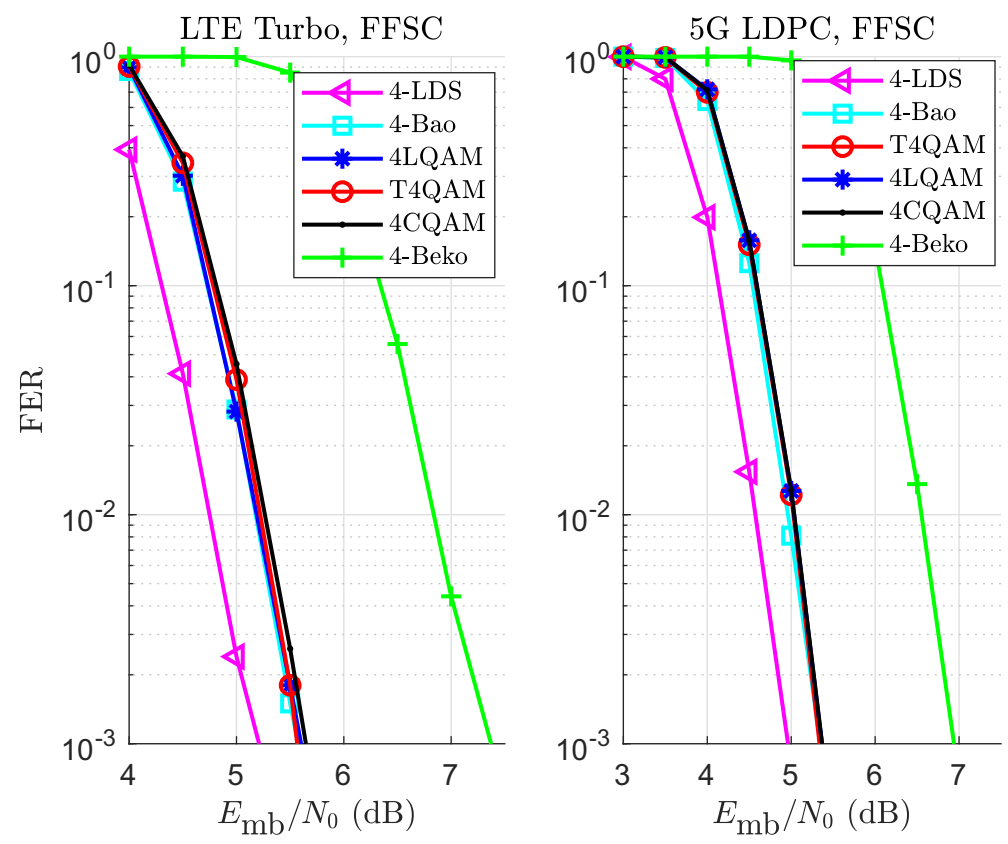

Figure C5: FER performance of LTE turbo-coded and 5G-compliant LDPC-coded systems with $R=4 / 5$ over FFSC (Case 3 ). 

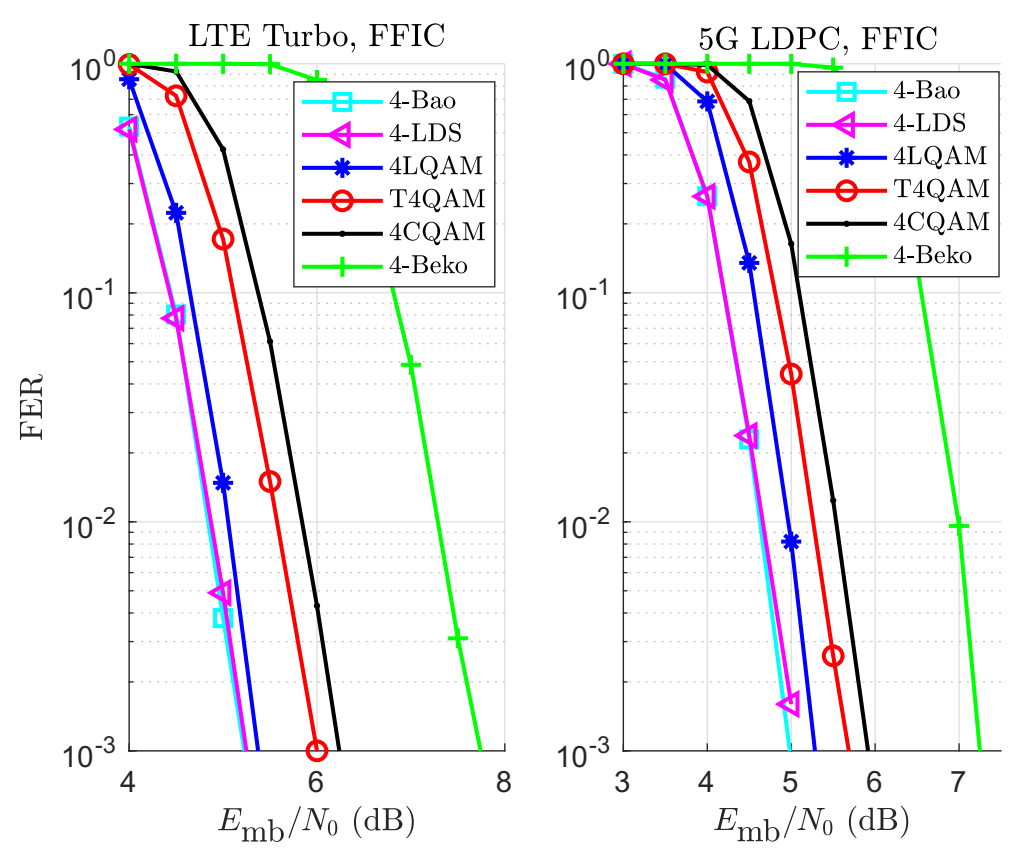

Figure C6: FER performance of LTE turbo-coded and 5G-compliant LDPC-coded systems with $R=4 / 5$ over FFIC (Case 4 ).
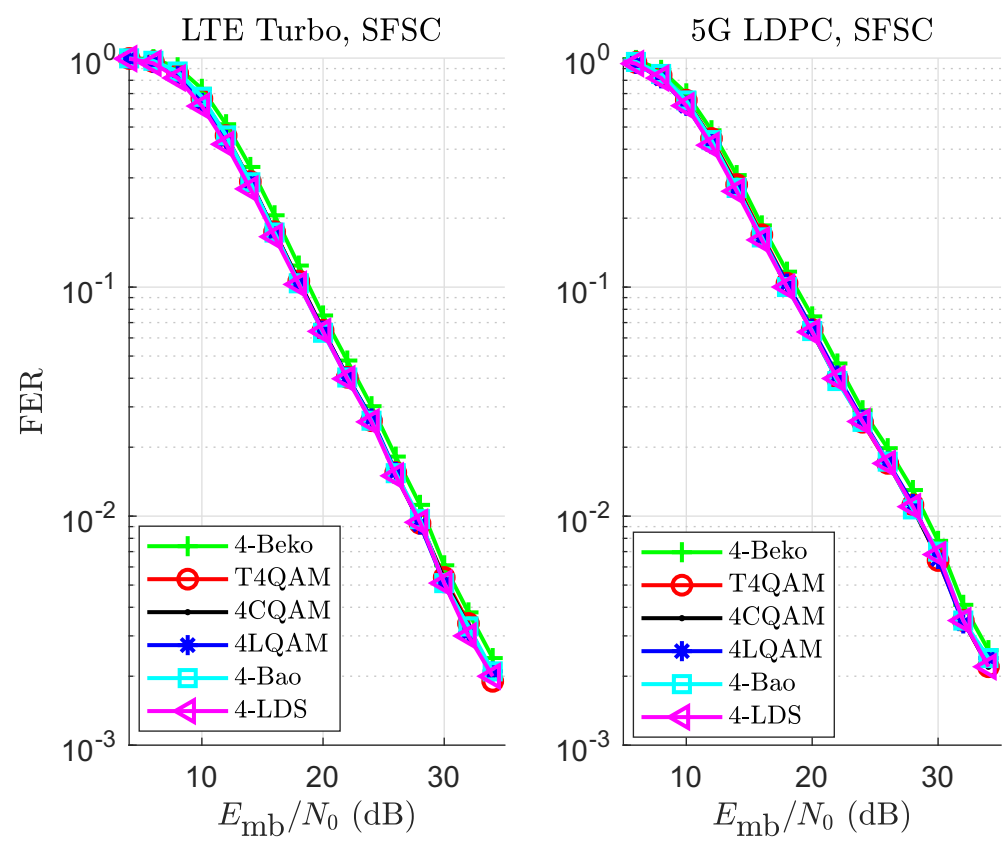

Figure C7: FER performance of LTE turbo-coded and 5G-compliant LDPC-coded systems with $R=4 / 5$ over SFSC (Case 5 ). 

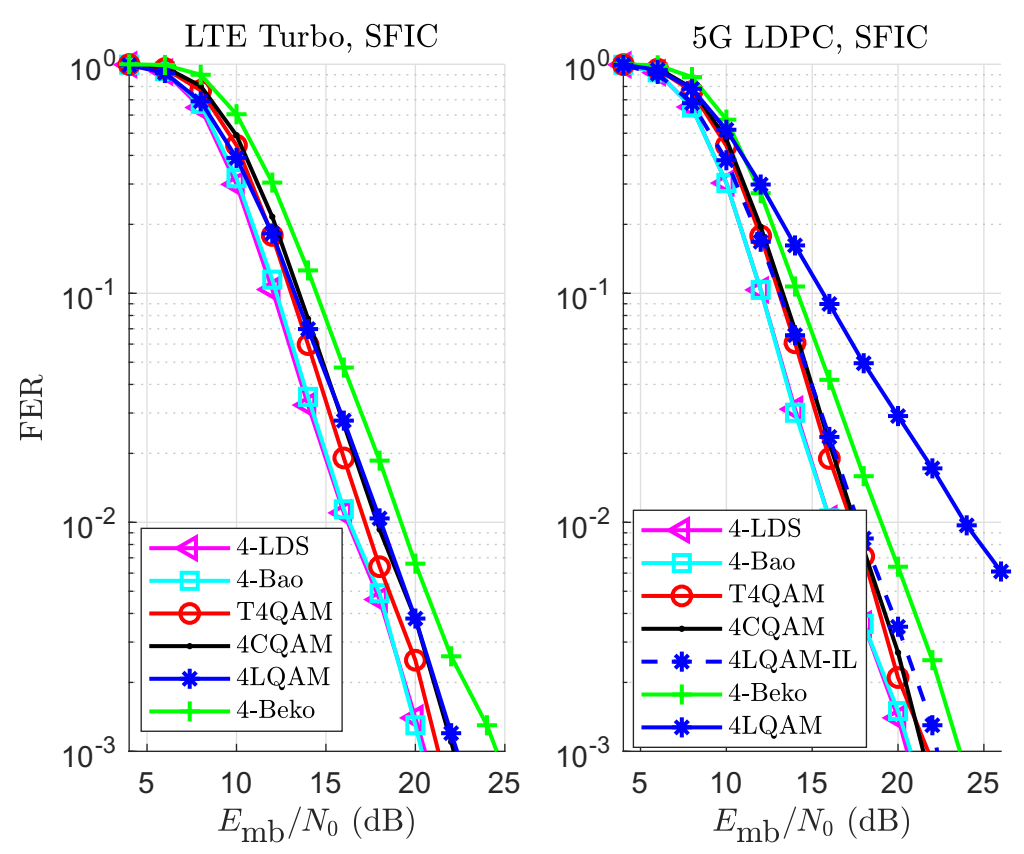

Figure C8: FER performance of LTE turbo-coded and 5G-compliant LDPC-coded systems with $R=4 / 5$ over SFIC (Case 6 ).

To explain the deviation from the expected behaviour, we observe, by referring to Fig. 3.4(a) that the four points of 4LQAM are mapped to only $N_{d}=2$ distinct points on each projection. In particular, one bit is used to specify the symbol transmitted over one RE, and the other bit is used independently for the symbol over the other $\mathrm{RE}$. This is in general an undesirable property for a constellation because if one RE is in a deep fade, it is impossible to recover the information from the other RE without relying on the redundancy of a powerful low-rate code. This is apparent in Fig. C5Fig. C8 that the 4LQAM constellation is a poor choice when a rate $4 / 5$ code (either turbo or LDPC) is used. When a rate $1 / 3$ code is used instead, this property of the constellation is less important, as shown by the relatively better performance of 4LQAM. However, in the SFIC scenario, where each user only sees two different fading coefficients, if there is a deep fade, not only will the user lose half of the code bits, but they will alternate between bits received over the faded RE and bits received over the unfaded one. The LTE turbo code, with its associated interleavers and rate-matching, is resilient to this phenomenon, but the inherent structure associated with the lifted 5G-compliant LDPC code is unable to deal with alternating "good/bad" bits. To test this hypothesis, we placed a pseudorandom interleaver between the encoder and the symbol mapper, and show the results for SFIC in Fig. C8, labelled as 4LQAM-IL. 
We can see that by including this interleaver, the performance of 4LQAM aligns with the prediction of the KPIs, similar to that of the LTE turbo code.

\section{C4 Conclusion}

We compared the effect of the 5G-compliant LDPC code with the LTE turbo code on the KPIs when designing MdCs for uplink SCMA systems under various channel scenarios. Simulation results show that the trends of MdCs in the presence of the 5G-compliant LDPC code are fairly consistent with their trends when the LTE turbo code is used, except for the 4LQAM constellation over one specific scenario. The performance of 4LQAM is notably worse than predicted by the KPIs when the lowrate $5 \mathrm{G}$-compliant LDPC code is used over SFIC. This phenomenon is due to the low number of distinct points of 4LQAM, in which the inherent structure associated with the 5G-compliant LDPC code is unable to deal with it. To overcome this problem, we used a pseudorandom interleaver between the 5G-compliant LDPC encoder and the symbol mapper. 